\title{
MÉTHODES D'INVESTIGATION DE L’ALIMENTATION ET DES MANGEURS
}

Sous la direction d'Olivier Lepiller, Tristan Fournier, Nicolas Bricas et Muriel Figuié

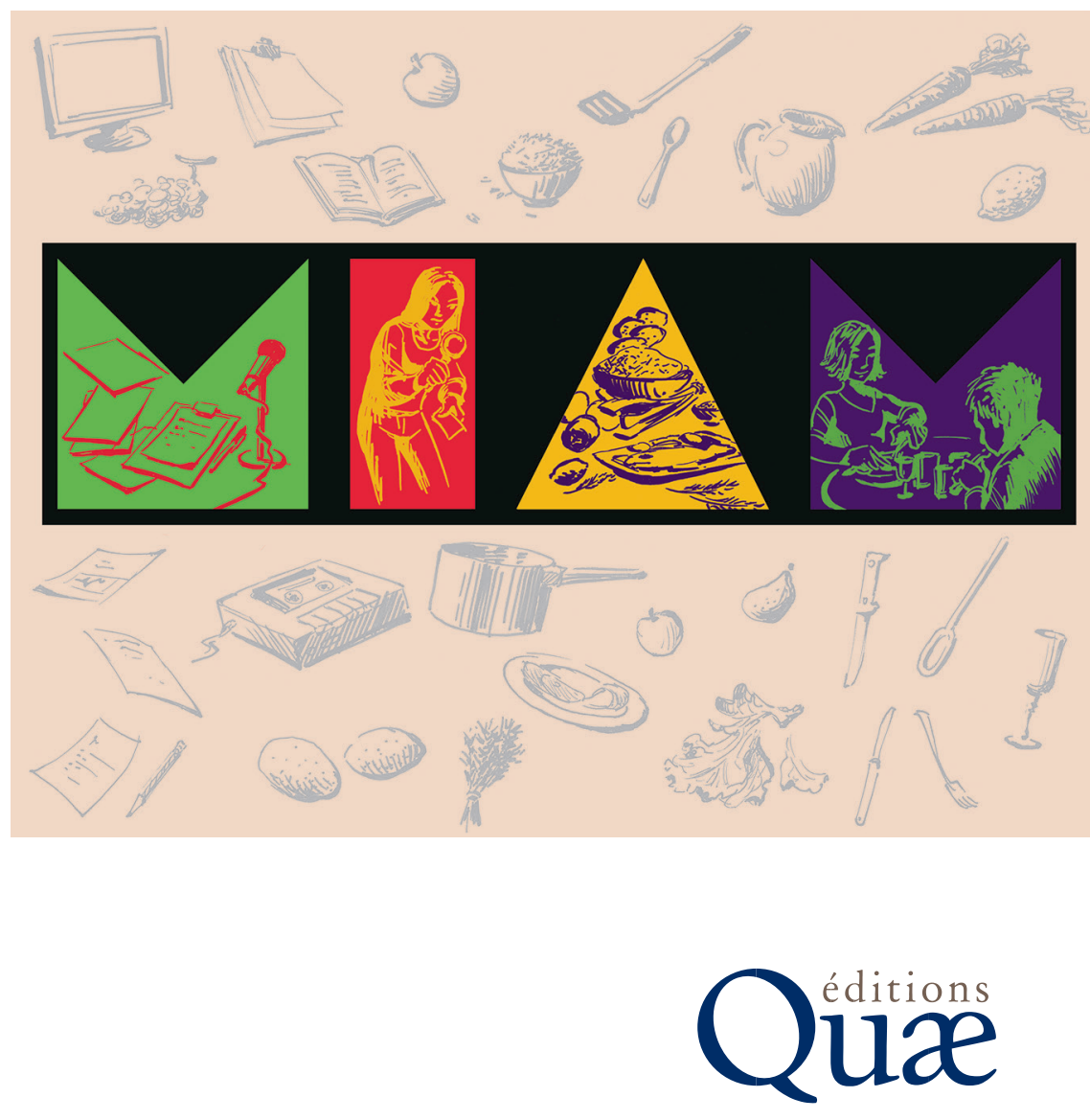





\section{Méthodes d'investigation de l'alimentation et des mangeurs - MIAM}

Sous la direction d'Olivier Lepiller, Tristan Fournier, Nicolas Bricas et Muriel Figuié 


\section{Collection Update Sciences \& Technologies}

Eating in the city

Socio-anthropological perspectives from Africa, Latin America and Asia

A. Soula, C. Yount-André, O. Lepiller,

N. Bricas, J-P. Hassoun, coord., D. Manley

(traduction)

2021, 158 p.

La santé globale au prisme de l'analyse des politiques publiques

S. Gardon, A. Gautier, G. Le Naour,

O. Faugère, R. Payre, coord.

2020, 248 p.

Manger en ville

Regards socio-anthropologiques d'Afrique, $d^{\prime}$ Amérique latine et d'Asie

A. Soula, C. Yount-André, O. Lepiller,

N. Bricas, J-P. Hassoun, coord.

2020, 172 p.
La question des échelles

en sciences humaines et sociales

S. Boulay, S. Fanchette, coord.

2019, $160 \mathrm{p}$.

Agroforesterie et services écosystémiques

en zone tropicale

Recherche de compromis

entre services d'approvisionnement

et autres services écosystémiques

J. Seghieri, J.-M. Harmand, coord.

2019

Systèmes agraires et changement climatique au sud

Les chemins de l'adaptation

H. Cochet, O. Ducourtieux, N. Garambois, coord.

2019, 282 p.

Les versions électroniques de cet ouvrage sont diffusées

sous licence Creative Commons CC-by-NC-ND 4.0

\section{(c) $(1) \Theta \Theta$}

\section{Pour citer cet ouvrage}

Olivier Lepiller, Tristan Fournier, Nicolas Bricas, Muriel Figuié (dir.), 2021. Méthodes d'investigation de l'alimentation et des mangeurs - MIAM. Versailles, éditions Quæ, 244 p. DOI : 10.35690/978-2-7592-3347-2

\section{Éditions Quæ}

RD 10, 78026 Versailles Cedex

www.quae.com - www.quae-open.com

○) Éditions Quæ, 2021 
Cet ouvrage prolonge un cycle de séminaires, organisé à Montpellier entre novembre 2017 et février 2020, dont nous tenons à remercier tous les participants. Ce cycle intitulé «Méthodes d'investigation de l'alimentation et des mangeurs» a fait l'objet d'un soutien de la Fondation Agropolis sous la référence ID 1700-025 à travers le programme «Investissements d'avenir» (Labex Agro : ANR-10-LABX-0001-01), dans le cadre de I-SITE-MUSE (ANR-16-IDEX-0006), et de l'UMR MoISA (Montpellier Interdisciplinary center on Sustainable Agri-food systems (Social and nutritional sciences) - Cirad, IAMM, INRAE, IRD, L'Institut Agro).

La publication de cet ouvrage a bénéficié des soutiens financiers de l'UMR MoISA, de l'École des hautes études en sciences sociales (EHESS), de l'Institut de recherche interdisciplinaire sur les enjeux sociaux (Iris), de la Chaire Unesco Alimentations du monde - L'Institut Agro, Cirad -, de l'Institut de recherche pour le développement (IRD) et du département Action, transitions et territoires (ACT) de l'Institut national de recherche pour l'agriculture, l'alimentation et l'environnement (INRAE). 



\section{Table des matières}

Une fiction introductive : quand les disciplines rencontrent les mangeurs

Nicolas Bricas, Olivier Lepiller, Tristan Fournier et Muriel Figuié

PARTIE 1

MESURER LES PRATIQUES ET LES REPRÉSENTATIONS DES MANGEURS

Chapitre 1. Les mesures de la consommation alimentaire individuelle :

des méthodes en fonction des objectifs visés

Edwige Landais et Diane Djossinou

Chapitre 2. La mesure de la sécurité alimentaire des individus et des ménages :

potentiel et défis de la collaboration entre sciences de la nutrition et sciences sociales

Emmanuelle Bouquet, Alissia Lourme-Ruiz et Anne Bichard

Chapitre 3. L'évaluation d'impact des actions de prévention et de promotion

de la santé nutritionnelle

Marion Tharrey, Mathilde Savy, Marlène Perignon, Caroline Méjean et Nicole Darmon

Chapitre 4. L'économie expérimentale : révéler les préférences et les facteurs de décision des individus.

Douadia Bougherara, Laurent Muller et Sabrina Teyssier

Chapitre 5. La mesure du bien-être alimentaire subjectif : saisir comment les mangeurs

évaluent leur alimentation

Christophe Serra-Mallol et Mila Lebrun

PARTIE 2

SuIVRE LES MANGEURS ET LES ALIMENTS À LA TRACE

Chapitre 6. L'ethnocomptabilité : observer, compter et comprendre ce à quoi tiennent les mangeurs

Margalida Mulet Pascual

Chapitre 7. Photovoice : une méthode participative pour identifier les environnements

alimentaires du point de vue des habitants

Carolyn I. Auma, Michelle Holdsworth, et Rebecca Pradeilles 
Chapitre 8. Les narrations quantifiées : une méthode qui combine entretien et analyse statistique des dynamiques biographiques.

Grégori Akermann et Paul Coeurquetin

Chapitre 9. L'(auto-)biographie : une démarche compréhensive pour accéder

à la subjectivité des mangeurs

Amandine Rochedy et Tristan Fournier

Chapitre 10. La méthode follow-the-thing : pour une biographie sociale et spatiale

des aliments

Michaël Bruckert

Chapitre 11. Les enquêtes sociologiques auprès des jeunes mangeurs :

enjeux méthodologiques et épistémologiques

Anne Dupuy et Géraldine Comoretto

\section{PARTIE 3}

ApPrÉHENDER LA CONSTRUCTION COLLECTIVE DU FAIT ALIMENTAIRE

Chapitre 12. Urbal : une évaluation participative et qualitative de la durabilité

des innovations alimentaires

Olivier Lepiller, Élodie Valette, Alison Blay-Palmer, Denis Sautier, Michaël Bruckert,

Marlène Perignon, Nguyen Thi Tan Loc, Nguyen Thi Sau, Ophélie Roudelle et Amélie Wood

Chapitre 13. La recherche-action : un processus d'analyse et de transformation sociale pour l'accès à l'alimentation durable.

Pauline Scherer

Chapitre 14. Les ateliers-théâtre : rendre compte de l'expérience sensible de l'alimentation ....215

Estelle Fourat et Frédérique Jankowski

Chapitre 15. Les focus groups : étudier le fait alimentaire à travers

des mises en discussion thématiques

Amandine Rochedy et Sandrine Barrey

Liste des auteurs. 


\title{
Une fiction introductive : quand les disciplines rencontrent les mangeurs
}

\author{
NICOLAS BRICAS ${ }^{a}$, OLIVIER LEPILLER ${ }^{a}$, \\ TRISTAN FoURNIER ${ }^{b}$ ET MURIEL FIGUIÉc
}

\footnotetext{
a CIRAD, UMR MoISA, F-34398 Montpellier, France; MoISA, Université Montpellier, CIHEAM-IAMM, CIRAD, INRAE, Institut Agro, IRD, Montpellier, France.

${ }^{\mathrm{b}}$ CNRS - Iris, EHESS, Paris, France.

c CIRAD, UMR MoISA, Maputo, Mozambique; MoISA, Université Montpellier, CIHEAM-IAMM, CIRAD, INRAE, Institut Agro, IRD, Montpellier, France.
}

Lorsque Charlotte Dulien, sociologue à l'Institut de recherche sur l'alimentation, reçut le mail de l'Agence française de l'excellence scientifique, son cœur se mit à battre fort. Elle avait peiné à monter, un peu en catastrophe, une réponse à l'appel à projets de l'Agence. Sa proposition portait sur les facteurs d'évolution de la consommation de viande. Charlotte tenait à ce projet pour lequel elle avait réussi le tour de force de réunir, sur une même question, plusieurs chercheurs de diverses disciplines. Toutes et tous avaient donné leur accord, sans véritablement discuter des articulations et des conditions de participation. C'était de toute manière un sujet porteur et il n'y avait pas vraiment le temps. «On verra ça si on a le projet!», lui avaient dit plusieurs des chercheurs impliqués.

Et l'Agence, dans son commentaire, ne s'y était pas trompée. Le projet, jugé intéressant et novateur, était pré-selectionné, mais les évaluateurs invitaient les chercheurs à préciser les articulations disciplinaires et à mettre à profit les complémentarités et éventuelles contradictions des différentes approches méthodologiques. Charlotte était contente, mais on entrait dans le dur! Il allait falloir se mettre autour d'une table et commencer vraiment à discuter.

Un mois plus tard, Charlotte avait réussi le nouveau tour de force de trouver deux jours successifs pour réunir tous les participants. Elle avait identifié une salle qui permettait de disposer d'une grande table en carré, mettant tout le monde au même niveau. Elle avait préparé un exposé introductif sur la structure prévue du projet et avait recruté un post-doctorant, Max Logos, formé à l'histoire des sciences et à l'épistémologie, qui allait participer au projet dans l'axe «Épistémologie de l'interdisciplinarité du projet». «Bon, il faudra leur expliquer ce que ça veut dire, parce que je ne suis pas sûre que tout le monde comprenne!» avait dit Charlotte à Max. 
Le jour J, tous les responsables d'équipes étaient là : Charlotte et Max; Nathalie Vitamine, nutritionniste; Alan Smith, économiste; Marcel Lhomme, anthropologue et Fabienne Descartes, géographe. Après un café partagé, un tour de table des participants, Charlotte fit son exposé introductif et proposa de passer en revue chacun des axes de recherche du projet.

Le premier était intitulé «Décrire : état des lieux, données de base sur les consommations de viande». L'enjeu de la discussion était de définir le périmètre des données de consommation à recueillir. Nathalie Vitamine y avait réfléchi et proposa d'emblée que soient recueillies toutes les consommations d'aliments d'origine animale et d'aliments végétaux riches en protéines, en particulier les légumineuses «qui peuvent remplacer la viande». Marcel Lhomme ne put s'empêcher de demander avec un sourire goguenard : «Et le cassoulet, les saucisses lentilles et le chili con carne, tu les mets dans quelle catégorie : viande ou légumineuses?» On voyait déjà que chaque discipline, privilégiant une dimension de l'alimentation - la nutrition pour Nathalie Vitamine, la culture culinaire pour Marcel Lhomme - dessinait implicitement un périmètre de ce qui était pertinent et prioritaire à observer. Et sur ce terrain, la situation se compliqua quand il fallut aborder les mesures de consommation nécessaires à chacun : des quantités de produits en poids pour Nathalie, qui les convertirait en grammes de protéines, des dépenses en valeur économique pour Alan Smith. Fabienne Descartes demanda si on ne pourrait pas mesurer des fréquences de consommation, mais se vit opposer que ce n'était pas assez précis. Elle ajouta alors que, quelle que soit la méthode de mesure, elle souhaitait que soient recueillies les origines géographiques des produits, mais que c'était difficile à faire. Marcel Lhomme voulait, lui, s'assurer qu'il pourrait identifier quels plats étaient mangés et précisa qu'il ne voulait pas se limiter aux aliments riches en protéines, argumentant qu'on pouvait «remplacer un plat de viande par une tarte aux épinards lors d'un dîner». Mais surtout, il voulait pouvoir interroger des «mangeurs et pas des consommateurs», et ce avant qu'on ne leur fasse passer un questionnaire qui leur suggérerait beaucoup trop quelle dimension de l'alimentation était privilégiée dans l'enquête. «Tu comprends, si ton questionnaire fait entendre aux gens que tu es nutritionniste, ils vont orienter leurs réponses sur les relations entre leur alimentation et la santé et ça va me biaiser toutes mes réponses». On sentit un certain agacement face à cette dénonciation des biais liés au prisme disciplinaire et face à cette volonté de tout embrasser.

Pour faire redescendre la pression, Charlotte Dulien saisit la perche tendue par Marcel, l'anthropologue, pour dire un mot sur la distinction mangeur/consommateur : «C'est vrai que nous sommes un certain nombre ici à utiliser le terme de mangeur plutôt que celui de consommateur. Ce n'est pas une coquetterie intellectuelle! L'objectif est surtout de mettre l'accent sur le fait que l'alimentation ne se réduit pas à la seule étape de la consommation, qui est d'ailleurs ambiguë : s'agit-il de l'acquisition en général? De l'achat? De l'ingestion et de la digestion? De tout cela à la fois? Bien sûr, la consommation reste essentielle à considérer, mais il y a également tout un tas d'aliments et de pratiques qui échappent au marché. On peut ainsi manger ce que l'on produit, ce que l'on cueille, chasse, ce que l'on échange entre voisins ou avec sa famille. Et puis la figure du "mangeur" permet de resituer les individus que nous étudions par rapport à l'ensemble des autres acteurs du système alimentaire : les acteurs du champ à l'assiette, pour le dire vite. 
Mais aussi ceux qui tiennent des discours sur l'alimentation dans l'espace public : les médias, les politiques, les experts, les médecins, etc. Ces systèmes d'acteurs et d'activités incluent l'étape cruciale de la cuisine, qui transforme des denrées en aliments, et celle du partage des repas, qui est au cœur de la vie sociale. Le terme de "mangeur" met aussi davantage l'accent sur le sens, cela permet d'envisager les valeurs, les affects, les représentations, les logiques qui accompagnent l'acte de manger. D'ailleurs, quand ils prennent un repas ou un casse-croûte, les gens désignent en général cette activité par le verbe "manger". On ne dirait pas à un collègue "ça te dit qu'on aille consommer ensemble ce midi ?" Il y a enfin la dimension organique de l'acte de manger. On mange des aliments qui viennent de plantes ou d'animaux, et qui nourrissent notre corps. Ça nous insère dans une chaîne alimentaire et au sein du monde vivant. Si cela vous convient, je vous propose qu'on utilise ce terme durant ces deux journées de réunion. C'est bien le terme que nous avons utilisé dans la rédaction de notre projet. N'y voyez pas un prisme disciplinaire, mais plutôt un premier pas vers l'interdisciplinarité, une ouverture à l'ensemble des dimensions de l'acte de manger. D'ailleurs, à la base de cette figure du "mangeur", il y a un geste interdisciplinaire, une volonté de saisir l'acte de manger dans toute sa complexité biologique, psychologique, sociologique, anthropologique, économique, géographique, historique, politique, écologique. Je vous invite à relire un ancien numéro de la revue Communications dirigé par Claude Fischler (1979), en particulier l'article introductif, et un chapitre d'un livre de JeanPierre Poulain (2013) qui retrace l'émergence de cette figure du "mangeur" ».

Sur ce, l'heure du déjeuner sonna. En marchant vers le petit restaurant que Charlotte avait choisi non loin de la salle de réunion, les conversations bilatérales commencèrent pour tenter des compromis entre disciplines. Et Max entendit Nathalie Vitamine et Alan Smith, qui restaient un peu en arrière, discuter des sciences humaines, «toujours un peu paranos à se sentir mal comprises des approches plus dures!», «toujours à dire qu'on ne voit nos objets de recherche qu'au travers de nos petits bouts de lorgnette!» Mais ils reconnaissaient que Marcel Lhomme et Fabienne Descartes avaient l'air vraiment sympas quand même... Et puis, c'était vrai, finalement, qu'ils s'apprêtaient à manger, et pas à consommer, tous ensemble. À peine arrivé au restaurant, Max Logos prit des notes sur son carnet. Pour lui, l'enquête avait commencé et il avait déjà noirci des pages et des pages!

La seconde partie de la journée aborda le second volet «Comprendre et expliquer : les déterminants de la consommation de viande». Fabienne, la géographe, et Marcel, l'anthropologue, avaient profité du déjeuner pour échanger sur la façon dont ils voulaient travailler sur les déterminants. Ils attaquèrent d'emblée la discussion en expliquant qu'ils voulaient s'entretenir librement et de manière approfondie avec un échantillon de mangeurs pour identifier les raisons qu'ils donnaient à l'évolution de leur consommation de viande, tout en reconstruisant les circuits par lesquels les aliments avaient transité avant de se retrouver dans l'assiette. Nathalie, la nutritionniste, et Alan, l'économiste, se regardèrent, puis ce dernier prit la parole : «Tu veux enquêter un échantillon de combien de personnes?»-«Je ne sais pas, je dirais une trentaine?», répondit Marcel Lhomme. «Tu sais, je diversifie un maximum les profils des gens sur lesquels j'enquête et je m'arrête quand je n'entends plus de discours différents de ce que j'ai déjà entendu. Souvent, ça fait une trentaine. Mais bon, ça peut être un peu plus!», expliqua-t-il. 
Nathalie Vitamine fit un grand sourire et déclara que, pour publier un article dans sa discipline, il lui fallait de gros échantillons, si possible des milliers de gens, pour pouvoir identifier, par du calcul statistique, quels étaient les déterminants des consommations mesurées. Mais elle savait que ce projet ne pouvait pas prévoir un très gros échantillon et que quelques centaines pourraient suffire. Alan Smith renchérit en expliquant qu'il voulait mesurer s'il y avait des différences de consommation selon le revenu des ménages et qu'il lui fallait absolument un échantillon représentatif de la population de référence. Lui aussi, il lui fallait un gros échantillon! Marcel Lhomme et Fabienne Descartes regardaient Nathalie Vitamine et Alan Smith jeter des coups d'œil à Charlotte Dulien et commençaient à sentir qu'ils allaient réclamer un budget plus important pour mener leur grosse enquête. Ça allait compliquer leurs plans de recruter chacun un post-doctorant. Charlotte conclut la session en indiquant qu'on pourrait articuler «quali» et «quanti». Les entretiens approfondis et les observations ethnographiques - le «quali» - permettraient d'identifier des facteurs d'évolution de la consommation, facteurs qui pourraient être proposés comme modalités de réponse dans le questionnaire du volet «quanti». Mais on entendit une remarque un peu contrariée : "Donc il va falloir qu'on attende que le "quali" soit fini pour faire le "quanti"?» Charlotte poursuivit sans broncher et conclut : «Bon, il faudra qu'on discute aussi du budget avant demain soir! » Sur ce, la première journée s'acheva et Charlotte proposa que celles et ceux qui le voulaient aillent dîner ensemble dans un restaurant "à condition de parler d'autre chose que du projet!». «Un resto avec des plats végétariens, j'espère !», demanda quelqu'un.

Le lendemain matin, alors que le café était servi dans la salle de réunion, la discussion porta sur l'axe «Intervenir : construire et tester des moyens d'orienter la consommation». Charlotte, la sociologue, avait proposé cet axe de travail, sachant qu'il y avait un certain consensus entre les chercheurs pour considérer qu'une trop forte consommation de viande, au-delà des stricts besoins nutritionnels, posait divers problèmes : de santé, d'environnement, d'éthique et que les discours militants ambiants qui prônaient la réduction de la consommation de viande semblaient être reçus très différemment selon les catégories sociales. Elle proposait donc qu'au travers de l'analyse de réactions des mangeurs à des interventions expérimentales, existantes ou à monter dans le cadre du projet, on puisse mieux saisir leurs motivations, les freins, les leviers pour orienter cette consommation vers plus de «durabilité», comme il était convenu de dire. Cette fois, ce furent Nathalie Vitamine et Marcel Lhomme qui se retrouvèrent plutôt sur la même longueur d'onde. Tous deux défendirent une expérimentation à construire avec des citoyens, alors qu'Alan Smith et Fabienne Descartes voulaient tester une hypothèse issue de la littérature de leurs disciplines respectives. Les premiers défendaient la nécessité de ne pas donner aux chercheurs le monopole des recommandations pour l'action, mais, au contraire, de tester une posture où les savoirs et les tactiques des mangeurs devaient être valorisés. Ils avaient discuté au restaurant la veille au soir et conclu à l'intérêt de porter attention à la façon dont les mangeurs pensaient la question pour l'intégrer dans la construction du questionnaire. De fait, ils voulaient tester ensemble une recherche-action, basée sur des interventions, qui paraissait à la fois éthiquement plus adaptée et pragmatiquement plus facile à publier car les revues scientifiques étaient en ce moment friandes de ces protocoles. Alan Smith, lui, 
voulait tester les effets de variations de prix au travers d'une expérimentation où les participants devraient révéler leur consentement à payer plus pour des viandes de qualité plus durable par un dispositif qui les engagerait. Pour publier les résultats, il fallait qu'il innove du point de vue des techniques d'enchères et un papier australien récent lui donnait une piste nouvelle qu'il voulait absolument tester. Fabienne, la géographe, qui avait discuté avec Alan la veille au soir, se disait que cette expérimentation pourrait permettre de tester les effets de l'origine de la viande sur les consommations. Par ailleurs, elle voulait identifier si les évolutions de la consommation étaient ou non couplées à des évolutions des lieux d'achat de la viande. La tendance au «flexitarisme», notamment, bénéficiait-elle aux bouchers de proximité ? Comme Alan, ce qui l'intéressait était d'explorer une question restée sans réponse dans la littérature, un front de recherche! «Mais c'est un vrai problème de société ou c'est juste une question de scientifique?», demanda, faussement naïve, Nathalie Vitamine?

Pour le déjeuner, Charlotte Dulien avait prévu une surprise dans la salle de réunion. Elle s'était arrangée avec un ami qui travaillait dans un labo néerlandais pour se faire livrer des produits alternatifs à la viande : des mini-steaks à base de cellules souches animales; une sorte de petite saucisse faite de bactéries cultivées sur des tissus de champignons; des mini-brochettes de vers à soie; et des bouchées de protéines de sésame texturées d'un nouveau type, légèrement grasses, et similaires à s'y méprendre à des nuggets de poulet. Tout cela avait été cuisiné comme de la viande par une amie de Charlotte, cheffe cuisinière. Ces différents échantillons étaient servis dans de jolies assiettes en fibres de bambou et on pouvait les déguster avec une simple fourchette ou même directement avec les doigts. "Pas besoin de couteau!» déclara Alan Smith. «Bonne remarque!» acquiesça Charlotte Dulien. Et elle ajouta : «Est-ce qu'on peut encore parler de viande si ça ne se coupe pas au couteau?». Marcel Lhomme allait se lancer dans un exposé sur l'usage du couteau lors du repas selon les sociétés et au travers des âges, mais Alan, l'économiste, le coupa dans son élan en lançant, goguenard : «Ah ben, c'est bien une question de sociologue ça! On s'en fiche un peu tant que c'est mangeable, non?». «Tant que c'est de la protéine, comme diraient les nutritionnistes!», rétorqua Marcel en faisant un clin d'œil à Nathalie Vitamine. Et celle-ci renchérit en lançant à Alan : «Ça dépend si c'est un bien élastique ou si c'est une commodité... à couper, comme diraient les économistes!». «Bon, passez-moi la carte que je vois comment ils appellent ça», demanda Fabienne, la géographe. «Ils ne peuvent pas se passer de cartes les géographes!», répondit Marcel Lhomme. Max, l'historien des sciences et épistémologue, grattait son cahier comme un fou et Charlotte Dulien souriait de voir s'établir des relations d' «affinités disciplinaires à plaisanterie», concept qu'elle était en train d'inventer, mais qui était, se disait-elle, une condition indispensable au succès du projet.

L'après-midi fut consacré à un exposé de Max, qui présenta les options méthodologiques disponibles pour le projet. Ce moment devait alimenter les futures discussions sur l'organisation pratique du projet et les règles pour construire le budget définitif dans les semaines à venir. Max rappela d'abord que, selon la finalité de la recherche, les méthodes variaient. «C'est la question qui justifie la méthode. Et bien souvent, les controverses sur les méthodes sont en fait des dialogues de sourds entre des chercheurs qui ne se posent pas les mêmes questions ». 
Ainsi, il expliqua que, si l'on veut mesurer l'importance d'un phénomène, comme cela avait été discuté lors de la première demi-journée, on n'emploie pas les mêmes méthodes que si l'on veut comprendre la logique interne de ce phénomène ou que si l'on veut évaluer le poids relatif de ses différents déterminants. «Une enquête quantitative peut nous montrer par exemple que les riches très diplômés mangent moins de viande que les classes moyennes peu diplômées. Mais ça ne nous dit pas pour quelles raisons du point de vue des mangeurs. C'est ça que peut apporter une approche qualitative et compréhensive». Et les méthodes sont encore différentes si l'on veut intervenir, proposer, mettre en ouvre ou évaluer les effets d'une action. Marcel Lhomme intervint pour dire «Oui, O.K., le choix de la méthode dépend de la question que l'on se pose. Mais le choix de la méthode dépend aussi du temps et surtout du budget dont tu disposes. Une série d'entretiens approfondis et leur analyse peuvent se réaliser en quelques mois, voire en quelques semaines et ne coûtent pas très cher, alors qu'une grosse enquête quantitative peut prendre plus d'un an de montage, de réalisation et d'analyse des résultats ». Alan, l'économiste, ne put s'empêcher de blaguer : «Tu veux dire, Monsieur l'anthropologue, que la méthodologie est inféodée à l'économique...?»Et il ajouta avec un clin d'œil «... comme beaucoup de choses, voire comme presque tout !» Et Charlotte conclut : «Il faut trouver le bon compromis entre la posture de recherche souvent définie par sa discipline, la question que l'on se pose et les moyens dont on dispose. Et d'ailleurs, des compromis, il va falloir en trouver dans ce projet pour que tout le monde s'y retrouve!».

Max enchaîna alors sur des considérations épistémologiques qu'il annonçait ardues, mais pas inutiles, ce qui ne sembla pas enthousiasmer l'auditoire plus que cela, à en juger par les bâillements réprimés qui se multipliaient - il faut dire que le thermos de café était vide depuis déjà une bonne heure. Mais, après tout, Max était là pour faire réfléchir les participants sur leurs pratiques de recherche et jouer un rôle de poil à gratter de l'interdisciplinarité. «L'action d'objectiver, commença-t-il, est à la base de l'activité scientifique. Elle consiste en premier lieu à définir clairement les contours du phénomène étudié - ou objet -, puis à produire sur cet objet un discours qui repose sur des observations renseignées empiriquement par un travail de collecte de données, avant d'être analysées selon des critères logiques explicites. Ce discours est donc vérifiable : il est, dans le cas des approches expérimentales reproductibles, réfutable ou, dans le cas des autres approches, plausible, c'est-àdire logiquement cohérent et fondé sur des observations. Au sein des approches scientifiques de l'alimentation et des mangeurs, plusieurs méthodes d'objectivation peuvent être distinguées en fonction de leur rapport à la dimension émique des phénomènes étudiés. »

Max expliqua ensuite que ce concept de «émique» ne pouvait se comprendre sans celui, opposé, de «étique». «Éthique avec un h?» demanda Nathalie Vitamine. «Non, étique sans $\mathrm{h}$ » répondit Max, qui développa, en invitant son auditoire à lire un article de Jean-Pierre Olivier de Sardan (1998) sur le sujet : «Les concepts de "étique" et de "émique" ont été forgés par un certain Kenneth Pike sur la base de travaux en linguistique, puis repris en anthropologie, notamment par Marvin Harris. Cette distinction permet de mieux comprendre les postures de recherche des différentes disciplines. » 
Il poursuivit : «L'étique est propre à la science. Il peut s'entendre comme approche interprétative des phénomènes étudiés : une approche produisant un discours scientifique de description, de compréhension et d'explication. Pour produire ce discours, les scientifiques suivent des méthodes et des règles logiques bien identifiées. C'est ce qu'on appelle communément le "sens savant" ». Max donna des exemples de discours étique provenant de différentes disciplines, en expliquant qu'il s'agissait bien souvent des notions qui rendaient les chercheurs incompréhensibles pour le commun des mortels : «situation commensale», «densité nutritionnelle», «diversité alimentaire», «propension à payer», «triangle culinaire», «environnement alimentaire».

«À l'inverse, poursuivit Max, l'approche émique renvoie à ce qu'on désigne par l'expression de "sens commun". Il s'agit des discours exprimés par les enquêtés, des représentations exprimables et qui peuvent être reconstruites par l'enquêteur, ou encore des codes et normes socioculturels dont les enquêtés sont capables de rendre compte si le chercheur les y aide, car ils ne sont pas toujours très conscients ni facilement exprimables. Une norme sociale, par exemple, se caractérise par la sanction sociale liée à sa transgression. Elle peut être mise en lumière en demandant à l'enquêté de juger un comportement supposé transgressif. Par exemple, on peut demander à un enquêté ce qu'il penserait si un de ses invités refusait de manger le plat de viande qu'il a préparé. On cernerait alors les normes autour du végétarisme et du partage des repas dans sa société.»

Mais aussitôt après avoir exposé cette distinction entre l'étique et l'émique, et comme le font souvent les chercheurs en sciences humaines - «C'est une manie chez eux», s'amusa silencieusement Nathalie Vitamine -, Max précisa qu'il ne fallait pas voir celle-ci comme une opposition stricte. Au contraire, l'étique et l'émique étaient plutôt les deux pôles d'un continuum. Nathalie rebondit sur cette remarque : «d'ailleurs, est-ce que, finalement, nous n'avons pas tous affaire à l'émique ? Parce que nous étudions l'alimentation humaine, donc des êtres humains capables de parler et de produire du sens. On ne peut pas faire comme si ce n'était pas le cas».

Du fond de la salle, près du radiateur, Marcel Lhomme acquiesça, avant de lancer : «Je suis complètement d'accord, mais je crois que nous avons aussi tous affaire avec l'étique, même nous, les spécialistes des "sciences molles"! ». Max allait justement y venir. Il projeta un schéma, tiré d'un ouvrage sur les méthodes d'enquête pour étudier l'alimentation et les mangeurs (figure 0.1). Il poursuivit : «Toutes les démarches de production de connaissances à ambition scientifique, c'est-à-dire qui ont l'ambition de rendre compte des phénomènes étudiés, en permettant la comparaison, la généralisation et surtout la critique, produisent un discours étique. Et cela vaut aussi bien pour les sciences dites "dures" que pour les sciences humaines. Une approche qui ne s'intéresserait qu'à des phénomènes émiques - les représentations partagées au sein d'un groupe par exemple -, à supposer que ce soit vraiment possible, ne peut avoir de prétention scientifique que si elle forge ou emprunte un langage étique pour en rendre compte. Donc, tous autant que nous sommes dans cette salle, nous produisons de l'étique. C'est même un peu notre raison d'être et la justification de nos salaires. Ne pas du tout produire d'étique, ce serait par exemple rendre compte de discours émiques collectés sur le terrain sans définition claire de l'objet, sans aucune analyse ajoutée, sans aucune généralisation, 
sans aucune opération de traduction pour les rendre compréhensibles et interprétables par nos lecteurs, par nos collègues, voire par les enquêtés eux-mêmes. Autrement dit, cela reviendrait à ne produire aucun travail d'objectivation. Si l'on colle trop à l'émique, on ne généralise rien, on est juste un porte-parole. Donc, pour résumer, les méthodes et disciplines se situent différemment par rapport à l'étique et à l'émique. Certaines cherchent plutôt à prendre de la distance avec l'émique et à produire un discours le plus étique possible. On est ici plutôt du côté de l'épistémologie des sciences dites dures, de la mesure objective, de l'utilisation des outils statistiques, des protocoles expérimentaux. D’autres sont centrées sur l'émique et cherchent à en produire une objectivation compréhensive, à restituer les logiques qui animent les enquêtés. On est plutôt du côté de l'épistémologie des sciences humaines, du plausible plutôt que du réfutable».

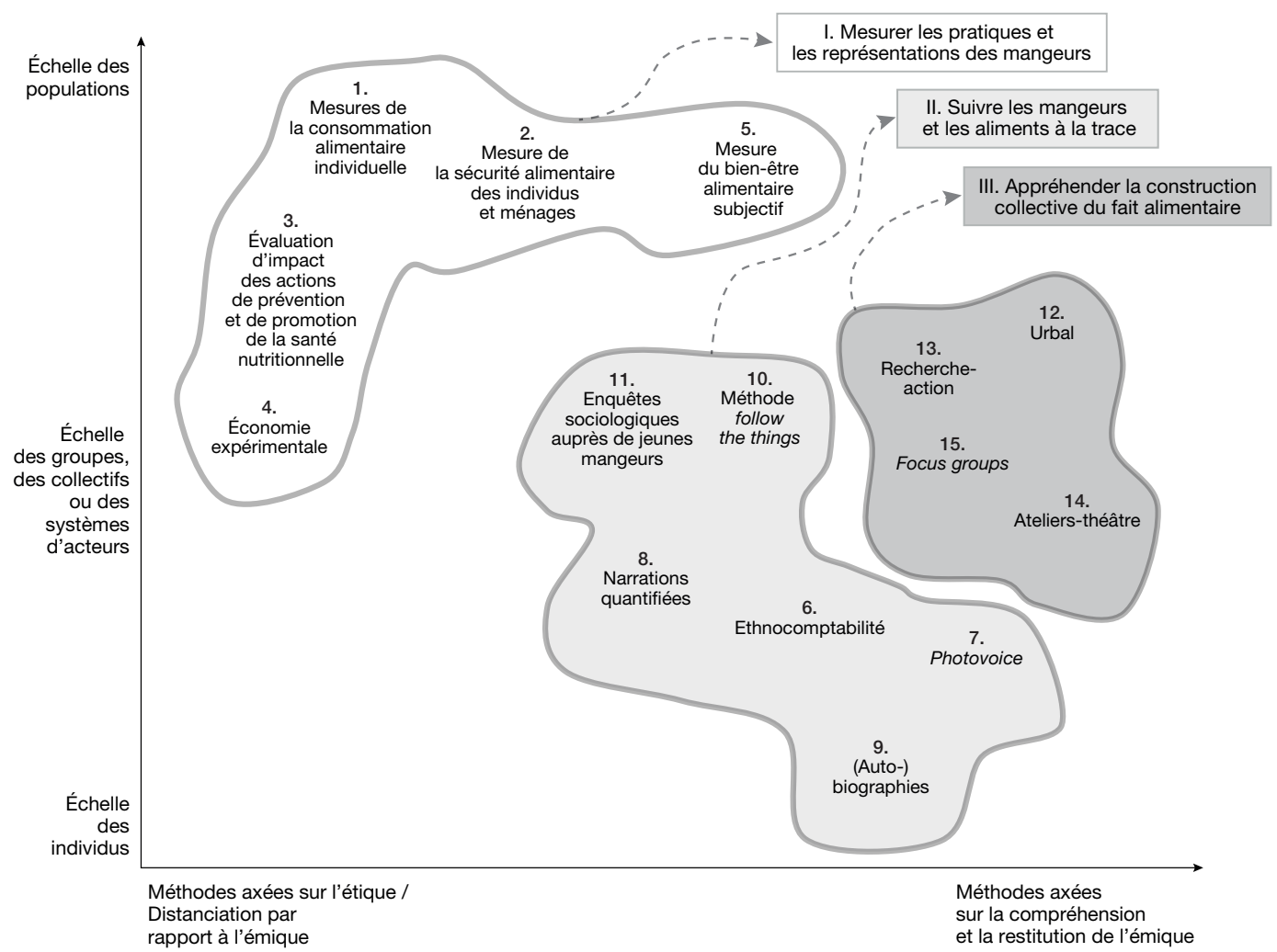

Figure 0.1. Carte cognitive des méthodes présentées dans les différents chapitres de l'ouvrage.

Après cette digression sur l'étique et l'émique qui sembla réveiller son auditoire, Max continua : «À côté de ce premier axe qui permet d'organiser les différentes méthodes d'investigation de l'alimentation et des mangeurs, on peut identifier un second axe, plus intuitif et plus évident, comme vous le voyez sur ce schéma : celui des échelles d'observation. Au plus fin, l'individu. Puis, il y a les groupes de différentes natures, définis de différentes manières par les différentes disciplines : la famille, le foyer, le ménage, l'unité de consommation, le clan, la tribu, le collectif, etc. 
Au-delà, il y a les populations, qui peuvent épouser les limites d'une région ou d'un État par exemple. Bien sûr, vous me direz que, parfois, pour généraliser à l'échelle d'un groupe, voire d'une population, on entre souvent par l'individu. Mais disons que ce qui compte ici, pour tenter d'organiser les méthodes, c'est l'échelle à laquelle s'intéressent en finalité les chercheurs, celle sur laquelle portent les connaissances qu'ils produisent. Aux échelles les plus fines, les méthodes qualitatives par entretiens et observations ethnographiques sont souvent privilégiées. La mise en œuvre de ces méthodes, qui visent une description détaillée est chronophage, si on prend en compte le rapport temps/enquêté. Par ailleurs, le budget qui leur est alloué au sein des projets de recherche est souvent limité». «Ne m'en parle pas!», lança Marcel Lhomme, tout sourire.

Max ajouta : «Avec ces méthodes qualitatives à des échelles fines, les échantillons ne sont pas pensés pour être représentatifs d'une population de référence. Ils le sont plutôt pour rendre compte de la diversité des situations, des types possibles. On cherche à interroger des personnes très différentes pour identifier l'ensemble des discours et des pratiques dont l'existence est a priori envisageable, même si certaines sont marginales dans la population, si elles ne pèsent pas statistiquement, avec le but de les comprendre, de restituer leurs logiques internes. L'histoire montre l'importance des marges : pensez, par exemple, à la consommation de viandes de qualité "biologique", complètement anecdotique il y a à peine trente ans, aujourd'hui bien plus importante. On peut identifier des déterminants des discours et des pratiques des mangeurs mais on ne peut pas faire d'analyse statistique pour mesurer leur importance relative. Les statistiques peuvent être utilisées éventuellement pour analyser les discours : fréquence d'apparition des mots ou des expressions par exemple. Aux échelles plus larges, celles de la population, il devient possible d'évaluer l'importance relative de ce qui a pu être identifié à une échelle plus fine. Ainsi, par exemple, on peut identifier par des entretiens approfondis auprès d'individus que les raisons d'une réduction de la consommation de viande peuvent être d'ordre sanitaire, économique, environnemental, éthique, gustatif, culturel, social, ou encore relatives au bien-être animal, raisons qui permettent de se différencier ou au contraire d'être intégré au sein d'un groupe social. Les entretiens permettent aussi de montrer comment ces différentes raisons de manger moins de viande s'associent et, éventuellement, comment leur association détermine un changement de pratique. Un événement biographique, l'arrivée d'un enfant par exemple, peut rendre simultanément plus sensible à des questions sanitaires et environnementales, forçant la projection dans l'avenir de la génération suivante. Une enquête quantitative permettra de mesurer l'importance relative de chacune de ces raisons dans une population, à condition que l'échantillon soit assez grand et suffisamment bien construit, et d'identifier comment ces raisons sont associées à des caractéristiques individuelles : âge, genre, niveau de vie, d'instruction, lieu de résidence, etc.».

Charlotte Dulien intervint alors : «Ces échelles d'observation différencient souvent les disciplines. Par exemple, les psychologues s'intéressent aux individus, même si c'est vrai que certains, en psychologie sociale ou culturelle, font aussi des enquêtes quantitatives. Les épidémiologistes privilégient les populations. Et ce qu'ils observent, chacun à leur échelle, n'est pas forcément cohérent. On verra par exemple de grandes différences de consommation de viande entre individus du fait 
de préférences gustatives, mais celles-ci se voient moins à l'échelle de populations où les facteurs économiques ou socioculturels jouent davantage. Pour autant, on peut quand même chercher à articuler des méthodes qui ont des échelles d'observation différentes. C'est là qu'il y a un véritable intérêt à l'interdisciplinarité.»

Nathalie Vitamine proposa un autre facteur de différenciation de démarches de recherche. D’un côté, elle identifiait des démarches qui privilégient l'avancée sur un front scientifique : explorer quelque chose que personne n'a encore exploré, un domaine mal connu, une méthode nouvelle. Ce qui compte est d'innover par rapport à ce que tous les autres ont déjà étudié. L'enjeu est de faire avancer la science. C'est cela qui permet plus facilement de publier, car c'est souvent cela que cherchent les revues. «Oui, mais tu vois, il y a plein de papiers qui explorent des nouvelles méthodes mais dont les résultats sont consternants de banalité sur leur utilité !», relativisa Charlotte Dulien. Nathalie reprit le fil de son propos en montrant qu'en effet, on pouvait aussi identifier des démarches qui partent de questions de société pour tenter d'y répondre. Ce qui compte est alors avant tout d'être utile à la résolution de problèmes sociétaux. Elle ajouta : «Cette distinction peut se résumer en parlant d'un côté de recherche fondamentale et, de l'autre, de recherche finalisée ». "Je suis d'accord, mais partir des questions posées par la société c'est risquer de s'enfermer dans un prisme imposé par certains de ses acteurs. Car souvent, le problème c'est justement la question, la façon dont elle est posée» déclara Fabienne Descartes. Et Charlotte répéta ce qu'avait dit Max plus tôt, à savoir qu'on assistait souvent à des débats scientifiques où s'affrontent des méthodes différentes alors qu'on ne se pose simplement pas les mêmes questions, avant de poursuivre : «Souvent, le choix de la méthode est fait en fonction de la question qu'on se pose, ou qui est posée socialement. Il faut alors se demander si la méthode choisie va bien permettre de répondre à la question. L'enjeu est alors avant tout la question et non pas la méthode. Mais il faut aussi reconnaître qu'une innovation méthodologique peut conduire à se poser de nouvelles questions. C'est parce qu'on peut mesurer de nouvelles dimensions, et qu'ainsi on les révèle, que l'on s'interroge sur leur rôle. C'est le cas, par exemple, avec les énormes possibilités de traitement offertes par les nouvelles méthodes du big data».

Charlotte, qui voyait l'heure tourner, profita de sa prise de parole pour remercier Max et conclure cette riche journée avec quatre remarques.

La première voulait que, pour s'entendre dans un groupe interdisciplinaire, il fallait accepter de relativiser l'importance que chaque discipline accorde aux dimensions de l'alimentation qu'elle étudie. Chacune tend à défendre l'importance de «sa» dimension privilégiée - sociale, biologique, culturelle, hédonique, écologique, psychologique, économique... - et a tendance à considérer les autres dimensions comme secondaires par rapport à celle sur laquelle elle focalise son regard. Par exemple, la nutrition considère la santé comme une dimension primordiale de l'alimentation et que l'on peut étudier le plaisir, les contraintes économiques, les normes sociales ou les usages culturels dans la façon dont ils influencent la santé nutritionnelle. Les sociologues et les anthropologues étudient les interactions sociales et les dynamiques culturelles qu'ils considèrent comme sur-déterminant les comportements qu'ils observent, alors que les économistes privilégient les contraintes matérielles et monétaires dans leur recherche de déterminants. L'interdisciplinarité suppose donc de reconnaître cette pluralité de 
points de vue et d'accepter que toutes les disciplines ne partagent pas une vision unifiée des finalités des comportements, qu'elles «zooment» sur certaines plutôt que d'autres, et tendent à les hiérarchiser.

La seconde remarque était inspirée de l'expérience de pensée de la carte à l'échelle $1 / 1$, celle qui se superpose complètement au territoire, telle qu'imaginée par Lewis Caroll, Jorge Luis Borges et Umberto Eco (Palsky, 1999). Une telle carte est la représentation la plus fiable du territoire, mais seulement voilà, elle est inutilisable! Il faut donc accepter de perdre de l'information pour rendre compte de la réalité et la sélection de l'information que l'on collecte grâce à la méthode utilisée dépend de son cadre conceptuel.

La troisième remarque s'appliquait à toutes les méthodes d'enquête, aussi bien qualitatives que quantitatives. Elle portait sur la nécessité de prendre en compte, d'une part, les effets d'interaction entre enquêteurs et enquêtés et, d'autre part, les effets de l'ordre et de la formulation des questions sur les réponses. Charlotte recommanda à tout le monde, quelle que soit sa discipline, de lire une sélection d'auteurs qu'elle considérait comme incontournables sur les questions de méthode : JeanPierre Olivier de Sardan (2012) et son ouvrage La rigueur du qualitatif, qui s'adresse à tous ceux qui font des enquêtes sur l'humain, même quantitatives d'ailleurs; Norbert Schwarz et Seymour Sudman (1996), qui ont mené de nombreuses enquêtes pour montrer comment la question oriente la réponse; Luc Van Campenhoudt et al. (2017) plus généralement sur la rigueur de déploiement des méthodes en sciences sociales; les travaux du collectif de recherche Amira ${ }^{1}$ sur l'adaptation des méthodes d'enquêtes auprès des ménages dans différents contextes culturels, notamment en Afrique, et en milieux dits «informels» (Gastellu, 1979; Couty et Winter, 1983). Plus centrée sur l'alimentation, Charlotte recommanda aussi un livre de Jean-Pierre Poulain (2008) qui abordait les principales méthodes d'étude des pratiques et représentations alimentaires. Et sur le même sujet en anglais, quelques incontournables : Helen M. Macbeth et Jeremy MacClancy (2004); Jeff Miller et Jonathan Deutsch (2010); Ken Albala (2012); Anne Murcott et al. (2013); Janet Chrzan et John Brett (2016); Jacob A. Klein et James L. Watson (2016).

La quatrième et dernière remarque de Charlotte était une recommandation : celle de consulter un ouvrage utile à qui voulait travailler sur l'alimentation. Il s'agissait du livre dont était tiré le schéma projeté un peu plus tôt par Max : le MIAM, pour méthodes d'investigation de l'alimentation et des mangeurs. Pour elle, cet ouvrage permettait de passer en revue et de situer les unes par rapport aux autres diverses méthodes : participatives impliquant les enquêtés, compréhensives déployées à l'échelle d'individus et de groupes, plus explicatives, menées sur quelques individus jusqu'à de très larges échantillons de population.

«Pour présenter les différentes méthodes que les éditeurs scientifiques ont compilées à la suite d'un cycle de séminaires organisés à Montpellier entre 2017 et 2020, ils ont choisi de les organiser graphiquement selon les deux axes, dont Max vous parlait

1. Groupe de recherche pour l'Amélioration des méthodes d'investigation en milieux informels et ruraux d'Afrique, Asie et Amérique latine (Amira). Ce collectif réunissait principalement des chercheurs de l'Office de la recherche scientifique et technique outre-mer (Orstom), devenu Institut de recherche pour le développement (IRD). 
tout à l'heure : l'axe étique-émique en abscisses et celui de l'échelle d'observation en ordonnées. Bien sûr il y aurait d'autres possibilités, mais cela permet aux lecteurs de situer graphiquement chacune des méthodes et aussi d'organiser les quinze chapitres de cet ouvrage en trois grands ensembles regroupant chacun des méthodes qui partagent un même air de famille. Le premier ensemble s'intitule "Mesurer les pratiques et les représentations des mangeurs". Il s'agit surtout de méthodes quantitatives visant la représentativité statistique, ou cherchant à mesurer le poids de différents facteurs des comportements des mangeurs. Le second ensemble a pour titre "Suivre les mangeurs et les aliments à la trace". Il s'agit de méthodes qui appréhendent les dynamiques des pratiques, des représentations ou de la circulation des aliments, à des échelles infra-populationnelles pour la plupart. Le troisième ensemble s'intitule "Appréhender la construction collective du fait alimentaire". Il s'agit principalement de méthodes participatives impliquant les enquêtés dans la production de connaissances » (figure 0.1).

Charlotte poursuivit son commentaire du schéma, puis ouvrit la discussion car il y avait de nombreuses questions. Quelqu'un lança l'idée d'appliquer l'ensemble des quinze méthodes présentées dans l'ouvrage à l'étude de la consommation de viande. Tout le monde convint que ça permettrait assurément d'éclairer cet objet sous des angles très complémentaires, mais que l'enveloppe budgétaire de l'Agence française de l'excellence scientifique ne le permettrait pas. Charlotte saisit la balle au bond : «Pour ça, on demandera de l'argent à l'Europe dans notre prochain projet ! En attendant, ça vous dirait d'aller manger un morceau tous ensemble?»

\section{" Références}

Albala K., 2012. Routledge International Handbook of Food Studies, New York, Routledge, 406 p.

Chrzan J., Brett J. (éd.), 2016. Research Methods for Anthropological Studies of Food and Nutrition, 1-3, Oxford, New York, Berghahn Books, 820 p.

Couty P., Winter G., 1983. Qualitatif et quantitatif. Deux modes d'investigation complémentaires. Réflexion à partir des recherches de l'Orstom en milieu rural africain. Groupe de recherche pour l'amélioration des méthodes d'investigation en milieux informels et ruraux d'Afrique, Asie et Amérique latine (Amira), 47, Paris, Orstom, 95 p. [En ligne] http://www.documentation.ird.fr/hor/ fdi: 15287

Fischler C. (éd.), 1979. La nourriture. Pour une anthropologie bioculturelle de l'alimentation. Communications, 31, 224 p.[En ligne] https://www.persee.fr/issue/comm_0588-8018_1979_num_31_1 (dernière consultation le 24/08/2021).

Gastellu J. M., 1979. Mais où sont donc ces unités économiques que nos amis cherchent tant en Afrique? Stateco, 19, 11-32, [En ligne] http://horizon.documentation.ird.fr/exl-doc/pleins_textes/ pleins_textes_4/sci_hum/00187.pdf (dernière consultation le 24/08/2021).

Klein J., Watson J. L., 2016. The Handbook of Food and Anthropology, Londres, Bloomsbury Academic, $496 \mathrm{p}$.

Macbeth H. M., MacClancy J., 2004. Researching Food Habits: Methods and Problems, New York, Oxford, Berghahn Books, 242 p.

Miller J., Deutsch J., 2010. Food Studies: An Introduction to Research Methods, Oxford, Berg, 229 p. Murcott A., Belasco W., Jackson P., 2013. The Handbook of Food Research, Londres, New York, Bloomsbury Publishing, $681 \mathrm{p}$.

Olivier de Sardan J. P., 2012. La rigueur du qualitatif. Les contraintes empiriques de l'interprétation socio-anthropologique, Bruxelles, Academia, $372 \mathrm{p}$. 
Olivier de Sardan J.-P., 1998. Émique. L'Homme, 38 (147), 151-166.

https://doi.org/10.3406/hom.1998.370510

Palsky G., 1999. Borgès, Carol et la carte au 1/1. Cybergeo: European Journal of Geography, 106. https://doi.org/10.4000/cybergeo.5233

Poulain J.-P., 2008. Manger aujourd'hui : attitudes, normes et pratiques. Toulouse, éditions Privat, $235 \mathrm{p}$.

Poulain J.-P., 2013. De l'intérêt sociologique pour l'alimentation aux sociologies de l'alimentation, in Poulain J.-P., Sociologies de l'alimentation, Paris, Presses universitaires de France, 155-188. [En ligne] https://www.cairn.info/sociologies-de-l-alimentation--9782130619406-page-155.htm?contenu $=$ resume (dernière consultation le 24/08/2021).

Schwarz N., Sudman S., 1996. Answering questions: Methodology for determining cognitive and communicative processes in survey research, San Francisco, Jossey-Bass Publishers, 469 p.

Van Campenhoudt L., Marquet J. J., Quivy R., 2017. Manuel de recherche en sciences sociales, Paris, Dunod, 384 p. 

Partie 1

Mesurer les pratiques et les représentations des mangeurs 



\title{
Chapitre 1 \\ Les mesures de la consommation alimentaire individuelle : des méthodes en fonction des objectifs visés
}

\author{
EDWIGE LANDAIS ${ }^{a}$ ET DIANE DJOSSINOU
}

\footnotetext{
a MoISA, Université de Montpellier, CIRAD, CIHEAM-IAMM, INRAE, Institut Agro, IRD, Montpellier, France.

${ }^{\text {b } U n i v e r s i t e ́ ~ d ' A b o m e y-C a l a v i, ~ F a c u l t e ́ ~ d e s ~ S c i e n c e s ~ A g r o n o m i q u e s, ~ d e ́ p a r t e m e n t ~ d e ~ N u t r i t i o n ~ e t ~ S c i e n c e s ~}$ Alimentaires, Cotonou, Bénin.
}

Les trois méthodes de mesure de la consommation alimentaire présentées permettent d'évaluer la qualité de l'alimentation ou d'identifier des modèles alimentaires dans une population donnée. Ces méthodes quantitatives reposent sur un travail important de préparation de la collecte et de gestion des données qu'il est fondamental de ne pas sous-estimer.

"It is easy to ask what people eat, but finding an answer can be a daunting task" (Helsing, 1991)

La mesure de la consommation alimentaire et des ingérés en nutriments au niveau individuel est une discipline récente qui a vu le jour au début du $\mathrm{xx}^{\mathrm{e}}$ siècle, notamment grâce au développement des tables de composition des aliments permettant de convertir les aliments en nutriments et dont les premières publications sont attribuées à Percy et Vaquelin en France en 1818 (Church, 2006). On peut tout de même considérer qu'une forme plus rudimentaire de mesure de la consommation alimentaire existe depuis l'Antiquité. Déjà à cette période, le repas est un moment du quotidien que les artistes s'attachent à immortaliser, notamment sur des poteries, et l'on a par exemple retrouvé des vases d'environ 460 av. J.-C. sur lesquels étaient représentés des banquets. Ainsi, au cours de l'histoire de l'humanité, ce que l'on mange et la façon dont on mange ont toujours été représentés de manière picturale au travers des siècles, notamment sur des poteries, des tapisseries ou encore sous forme de peintures. Au-delà de ces représentations picturales, il existe également depuis l'Antiquité des textes sur l'art culinaire, ainsi que des livres de recettes, dont notamment le De re coquinaria, datant du $\mathrm{IV}^{\mathrm{e}}$ siècle apr. J.-C, compilation de recettes romaines. L'arrivée du papier et de l'imprimerie à partir du XIV e siècle va par la suite permettre la diffusion des premiers livres de cuisine et la connaissance précise des types de plats préparés et consommés aux différentes époques. Par exemple, dans un livre de recettes datant du XVIII ${ }^{\mathrm{e}}$ siècle, on peut lire une description de préparation de pâtes 
au bouillon : «On fait un bouillon de viande sans herbes, avec du sel, et quand il est fait on met dans un plat sur un peu de feu la quantité de pâtes qu'on juge à propos ; à mesure qu'elle s'échauffe, on l'arrose doucement de bouillon, la pâte s'imbibe et se gonfle, et lorsqu'on la voit à un certain degré de grosseur ou sans se dissoudre elle est tendre, molle et bien humectée, on la sert sur la table» (Flandrin, 1983). De même, certains registres (archives de communauté, actes notariés de pensions, registres de versements de salaires en nature) permettent d'avoir une description quantitative de la consommation de certains aliments. Ainsi, grâce à ce type de registre, on sait par exemple que «Le Provençal des XIV et XV siècles boit beaucoup de vin (210 l par an), mange de bonnes quantités de pain (300 kg par an). Le mets fondamental est une soupe aux choux et au lard ou une soupe de fèves ou de lentilles ou encore un bouillon de viande dans lequel on trempe la grosse ration de pain. Un peu de poisson salé et de porc salé, un peu de viande fraîche peut-être deux fois par semaine (la consommation atteint $26 \mathrm{~kg}$ par an en 1473)» (Benassar et Goy, 1975). Les méthodes de mesure de la consommation individuelle telle qu'on les connaît aujourd'hui n'ont fait leur apparition qu'autour des années 1940 (1942 pour le rappel des 24 heures et 1947 pour l'histoire diététique ${ }^{2}$ ) (Biro et al., 2002). Ces méthodes sont généralement divisées en deux types : les méthodes rétrospectives visant à mesurer la consommation passée, comme les méthodes basées sur les questionnaires de fréquence de consommation ou la méthode du rappel des 24 heures; et les méthodes prospectives visant à mesurer la consommation actuelle, comme les enregistrements alimentaires.

\section{" Plusieurs types de méthodes en fonction des objectifs de mesure}

En tant que déterminant de l'état nutritionnel et de la santé, la consommation alimentaire est une préoccupation majeure des Objectifs de développement durable adoptés par les Nations unies; elle est associée à des domaines prioritaires comme le développement de l'agriculture durable, la production et la disponibilité des aliments ou encore la couverture des besoins nutritionnels et la santé des populations. Cependant, la consommation alimentaire est complexe à appréhender. Le choix de la méthode utilisée va dépendre notamment de l'objectif de l'étude (Webster-Gandy et al., 2020). Il est cependant important de garder à l'esprit qu'aucune de ces méthodes ne permet une évaluation exacte de la consommation alimentaire « réelle».

Dans le présent chapitre sont décrites les trois méthodes les plus couramment utilisées en enquête en population : deux méthodes rétrospectives (la méthode des fréquences de consommation et le rappel des 24 heures) et une méthode prospective (la méthode des enregistrements alimentaires).

\footnotetext{
2. L'histoire diététique est une méthode - non détaillée dans ce chapitre -, qui vise à reconstruire, avec l'enquêté dans le cadre d'entretiens et sur une période donnée, l'histoire de ses habitudes alimentaires (répartition des repas et des prises hors-repas, aliments fréquemment consommés, goûts et préférences, etc.), de ses problèmes ou observations liés à l'alimentation (troubles du comportement alimentaire, intolérances, allergies, maladies métaboliques, signes digestifs...), de ses pratiques de soins liés à l'alimentation (prises en charge médicales, consultations diététiques et nutritionnelles, etc.).
} 
Ces trois méthodes peuvent permettre le calcul de différents indices alimentaires qui vont rendre compte notamment de la qualité de l'alimentation (e.g. Diet Quality Index-International, Healthy Eating Index...), mais vont aussi permettre de caractériser des modèles alimentaires (e.g. régime méditerranéen, régime occidental...). Le rappel des 24 heures, en plus de cela, rend également possible le calcul des scores de diversité alimentaire tel que le Dietary Diversity Score, qui rend compte de l'adéquation nutritionnelle.

\section{Méthodes rétrospectives}

\section{- Mesure de la fréquence de consommation des aliments}

Cette méthode, via un questionnaire, permet d'évaluer, par individu, la fréquence de consommation des aliments ou groupes d'aliments sur une période de temps donnée (généralement une semaine ou un mois, mais parfois sur une période plus longue pouvant aller jusqu'à un an). Elle repose sur l'administration d'un questionnaire de fréquence, incluant une liste d'aliments (ou groupes d'aliments), et des catégories de réponse de fréquence correspondantes, par exemple «jamais, une fois par semaine, deux fois par mois ». Les modalités de réponse doivent garantir que toutes les catégories de temps sont incluses pour la période cible, c'est-à-dire qu'il n'y a pas de lacunes. Le nombre et les types d'aliments présents dans le questionnaire dépendent de l'objectif de l'étude pour lequel le questionnaire a été élaboré, en lien avec la population étudiée (femmes enceintes, personnes âgées, jeunes enfants, population générale, par exemple). Par conséquent, le questionnaire peut ne contenir que quelques items, par exemple lorsqu'il se concentre sur des nutriments particuliers (tels que les fibres alimentaires ou le fer), ou il peut en contenir plus, par exemple jusqu'à 200 items lorsqu'il vise l'évaluation du régime alimentaire dans son ensemble (c'est-à-dire apport énergétique et ingérés en nutriments). En général, les aliments (ou groupes d'aliments) inclus dans le questionnaire doivent être informatifs. C'està-dire que chaque aliment doit être consommé assez fréquemment par un nombre non négligeable de sujets; contenir en quantité suffisante le nutriment/l'aliment dont l'apport est étudié; être consommé en quantité (fréquence) variable selon les individus pour que le questionnaire soit discriminant.

Le questionnaire de fréquence a été conçu à l'origine pour fournir des informations qualitatives descriptives sur les habitudes de consommation alimentaire. Avec l'ajout de la taille des portions, le questionnaire de fréquence est devenu semiquantitatif (lorsque les quantités consommées sont estimées à l'aide de portions standards ou de référence) ou quantitatif (lorsque les quantités consommées sont estimées à l'aide de mesures ménagères du type cuillère, bol, verre, ou de photographies de taille de portion d'aliments). La quantification permet ainsi de calculer des ingérés alimentaires (en multipliant la fréquence par la quantité).

Le principe sous-jacent du questionnaire de fréquence est de renoncer à la mesure précise de l'apport alimentaire au profit d'informations plus brutes concernant une période de temps prolongée. Ainsi, l'approche par questionnaire de fréquence vise à mesurer davantage le régime alimentaire habituel que l'apport réel précis. Le questionnaire de fréquence est généralement conçu pour classer les individus dans de 
grandes catégories (par exemple en terciles), plutôt que pour calculer des apports moyens exacts. Il est principalement utilisé pour évaluer les associations entre les habitudes alimentaires et le risque de maladies dans le cadre d'études de cohortes ou de cas témoins.

Le questionnaire de fréquence constitue un moyen relativement peu coûteux et standardisé de collecter des données auprès d'un grand nombre d'individus. Il peut facilement être auto-administré (si les répondants savent lire et écrire) ou même administré par ordinateur. Les données peuvent être facilement traitées et informatisées. La plupart des questionnaires peuvent être remplis relativement rapidement - selon la longueur de la liste des aliments - et prennent généralement entre 15 et 30 minutes à remplir, ce qui représente une faible charge pour les répondants et conduit donc à une meilleure conformité.

L'un des principaux inconvénients du questionnaire de fréquence est que sa mise au point et sa validation nécessitent à la fois d'avoir déjà des données alimentaires pour la population cible et de mettre en place des études de validation (comparaison avec les résultats obtenus par une méthode standard comme des biomarqueurs sanguins ou une enquête par pesée), qui sont très longues et lourdes (Cade et al., 2002). Un autre inconvénient de cette méthode est sa faible capacité à obtenir des informations sur la façon dont les aliments sont consommés (par exemple, les méthodes de cuisson), ni sur les combinaisons d'aliments au sein d'un repas. En outre, l'apport moyen dépend du nombre d'items alimentaires, c'est-à-dire que plus la liste des aliments est longue, plus l'apport risque d'être surestimé (inversement, plus la liste est courte, plus l'apport risque d'être sous-estimé).

\section{- Rappel des 24 heures}

Le rappel des 24 heures est la méthode la plus utilisée pour obtenir des données quantitatives dans les enquêtes en population. Cette méthode consiste généralement en un entretien en face-à-face (ou parfois en un entretien téléphonique), mené par un enquêteur qualifié, au cours duquel la personne interrogée est invitée à fournir des informations détaillées sur tout ce qu'elle a bu et mangé au cours de la journée précédant le jour de l'enquête. Au cours de l'entretien, afin de recueillir des informations complètes et précises, l'enquêteur utilise des questions ouvertes et des questions d'approfondissement, maintient une attitude neutre vis-à-vis des réponses et évite les questions suggestives ainsi que les commentaires critiques.

La technique d'entretien à passages multiples en quatre étapes est souvent utilisée et reste à privilégier. Tout d'abord, la liste complète des aliments et boissons consommés par le sujet au cours du jour précédant l'enquête est établie. Ensuite, une description détaillée et précise de chaque aliment et boisson consommés (y compris les méthodes de préparation et de cuisson des aliments, la marque des produits commerciaux) est recueillie. Puis des estimations de la quantité d'aliments et de boissons consommés sont obtenues, généralement à l'aide de mesures ménagères ou de photographies d'aliments. Des informations sur les ingrédients des plats composés (recettes) consommés par la personne interrogée doivent également être collectées à ce stade. Enfin, le questionnaire de rappel est passé en revue afin de s'assurer que tous les aliments ont été correctement enregistrés. 
Dans l'idéal, le rappel doit être effectué à l'aveugle (c'est-à-dire que la personne ne doit pas savoir à l'avance le jour sur lequel portera le rappel) et au domicile de la personne enquêtée car cela encourage la participation, améliore la qualité des informations et facilite les étapes de calibration des mesures ménagères utilisées pour les quantifications.

Habituellement, les personnes interrogées sont les sujets de l'enquête de rappel eux-mêmes. Dans certains cas, lorsque le sujet ne peut pas répondre directement luimême, par exemple les enfants dont les réponses ne sont pas suffisamment fiables avant l'âge de huit ans (Arsenault et al., 2020) ou les sujets souffrant de problème de mémoire, le répondant peut être soit un parent, soit la personne qui s'occupe d'eux.

Le rappel des 24 heures permet d'évaluer de manière assez précise les ingérés alimentaires des individus et peut être utilisé pour estimer l'apport absolu plutôt que l'apport relatif. Par conséquent, si l'objectif de l'étude est de décrire la consommation habituelle d'un individu ou d'estimer la distribution de la consommation individuelle au sein de la population étudiée, un seul rappel est insuffisant (principalement en raison de la variabilité quotidienne). Dans ce cas, il est préférable de mener plusieurs rappels pour un même individu sur plusieurs jours non consécutifs afin de calculer des ingérés habituels. En cas de répétition, le rappel des 24 heures peut inclure à la fois des jours de semaine et des jours de week-end, en supposant des différences d'apport alimentaire entre les différents jours de la semaine. Idéalement, chaque jour de la semaine devrait être représenté de manière équivalente au sein de la population étudiée, mais en pratique, cela est généralement assez compliqué à obtenir en population.

Dans cette méthode, il n'est pas nécessaire que le répondant soit alphabétisé. C'est bien là l'une de ses forces. De plus, les entretiens nécessitent généralement une trentaine de minutes. Par conséquent, la charge du répondant est relativement faible et le taux de réponse est généralement élevé. Le rappel des 24 heures étant basé sur des questions ouvertes, cela permet un niveau de spécificité illimité concernant les descriptions des aliments (type d'aliment, méthodes de préparation, méthodes de cuisson, provenance des aliments, etc.). De même, cette méthode permet de recueillir de l'information sur la structuration des différentes prises alimentaires, ainsi que les combinaisons d'aliments au cours de ces dernières. Les principales limites associées au rappel des 24 heures sont sa dépendance à la mémoire de la personne interrogée, à la fois pour identifier les aliments et les boissons consommés, mais également pour évaluer les quantités consommées. Généralement, les ingérés mesurés via le rappel des 24 heures ont tendance à être sous-estimés avec des variations selon les participants, les femmes et les personnes en surpoids étant plus enclines à la sous-estimation de leur consommation (Thompson et Byers, 1994; Webster-Gandy et al., 2020).

\section{Méthode prospective : enregistrements alimentaires}

Lors d'un enregistrement alimentaire, il est demandé au participant de noter en temps réel sur un carnet le détail de toutes ses consommations (boissons comprises) au cours d'une période de temps déterminée. Historiquement, cette période s'étendait sur sept jours afin de capturer les variations au cours de la semaine et l'on parlait alors de semainier alimentaire. Ce type de méthode demandant un investissement important de la part du participant, en pratique la période de temps peut être dimi- 
nuée et les enregistrements alimentaires se font alors le plus souvent sur une période de trois jours. Généralement, les jours sont consécutifs et idéalement comportent un jour de week-end. Le participant peut quantifier sa consommation soit à l'aide d'une balance de cuisine - et dans ce cas on parle d'enquête par pesée -, soit à l'aide d'outils du type mesures ménagères ou photographies de taille de portion d'aliments. Pour ce type de méthode, il est nécessaire de former les participants pour qu'ils décrivent précisément les aliments (nom, type de préparation, type de cuisson...), ainsi que les quantités consommées. À la fin de la période d'enregistrement, un intervieweur qualifié passe en revue l'enregistrement avec le participant afin de clarifier et rechercher d'éventuels aliments oubliés.

L'enregistrement alimentaire permet d'avoir une description détaillée des aliments consommés, ainsi qu'une mesure précise des ingérés (cela est d'autant plus vrai que la quantification se fait par pesée). Si l'enregistrement est effectué sur une semaine, il permet également de capturer la diversité du régime alimentaire.

Un des inconvénients majeurs lié aux enregistrements alimentaires réside dans la nécessité que les participants soient alphabétisés, rendant notamment ce type de méthode difficilement applicable dans les pays du Sud. Par ailleurs, ce type de méthode demande un investissement fort de la part des participants, et ce d'autant plus que le nombre de jours est grand, pouvant entraîner d'une part un biais de sélection des participants, qui conduit à privilégier les plus motivés, et d'autre part une modification du comportement des participants allant généralement dans le sens d'une sous-déclaration (en quantité, principalement si les aliments sont pesés ou sur le type d'aliments, notamment les aliments consommés en dehors des repas) (Thompson et Byers, 1994; Webster-Gandy et al., 2020).

\section{Avantages et inconvénients des différentes méthodes}

Le tableau ci-dessous (tableau 1.1) récapitule les principaux avantages et inconvénients du questionnaire de fréquence, du rappel des 24 heures et des enregistrements alimentaires.

\section{Erreurs associées aux méthodes de mesure de la consommation alimentaire}

"There is not, and probably never will be, a method that can estimate dietary intake without error. » (Beaton, 1994)

Les erreurs sont de deux natures : aléatoires ou systématiques.

Les erreurs aléatoires (variabilité journalière des apports, estimation des quantités) augmentent la variance des ingérés alimentaires et donc réduisent leur précision. Les effets des erreurs aléatoires peuvent être réduits en augmentant le nombre d'observations. La variabilité liée aux spécificités éventuelles du jour de l'observation peut être réduite soit en augmentant le nombre de jours d'observation, soit en augmentant la taille de l'échantillon (Rutishauser, 2005).

Les erreurs systématiques peuvent être dues par exemple à l'utilisation d'une donnée incorrecte de composition nutritionnelle d'un aliment et conduiront donc 
Tableau 1.1. Avantages et inconvénients des trois méthodes présentées

\begin{tabular}{|c|c|c|}
\hline & Avantages & Inconvénients \\
\hline \multirow[t]{5}{*}{$\begin{array}{l}\text { Questionnaire de } \\
\text { fréquence (méthode } \\
\text { rétrospective) }\end{array}$} & $\begin{array}{l}\text { Pas de modification du } \\
\text { comportement des sujets } \\
\text { enquêtés par le dispositif } \\
\text { d'enquête }\end{array}$ & $\begin{array}{l}\text { Pas/peu de précision sur les aliments } \\
\text { (préparation, cuisson, marques) }\end{array}$ \\
\hline & $\begin{array}{l}\text { Charge/fardeau relativement } \\
\text { faible pour l'enquêté }\end{array}$ & $\begin{array}{l}\text { La coopération du répondant et la } \\
\text { précision de ses réponses diminuent avec } \\
\text { la longueur du questionnaire }\end{array}$ \\
\hline & Rapide à remplir & $\begin{array}{l}\text { Développement et validation du } \\
\text { questionnaire représentent une lourde } \\
\text { charge de travail }\end{array}$ \\
\hline & Auto-administration possible & $\begin{array}{l}\text { Les questionnaires longs surestiment les } \\
\text { ingérés et les courts les sous-estiment }\end{array}$ \\
\hline & $\begin{array}{l}\text { Possibilité d'une lecture } \\
\text { optique des réponses } \\
\rightarrow \text { diminution des coûts liés } \\
\text { à la saisie }\end{array}$ & $\begin{array}{l}\text { Peu précis pour évaluer l'ingéré calorique } \\
\text { et/ou en nutriments (d'où un classement } \\
\text { des individus par exemple en terciles } \\
\text { de consommation) }\end{array}$ \\
\hline \multirow[t]{5}{*}{$\begin{array}{l}\text { Rappel des } \\
24 \text { heures (méthode } \\
\text { rétrospective) }\end{array}$} & $\begin{array}{l}\text { Pas de modification } \\
\text { du comportement des sujets } \\
\text { enquêtés par le dispositif } \\
\text { d'enquête }\end{array}$ & $\begin{array}{l}\text { Le jour précédent n'est peut-être pas } \\
\text { typique }\end{array}$ \\
\hline & $\begin{array}{l}\text { Charge/fardeau relativement } \\
\text { faible pour l'enquêté }\end{array}$ & $\begin{array}{l}\text { Cette méthode ne rend pas compte } \\
\text { de la variabilité entre les différents jours } \\
\text { de la semaine, sauf si répétition }\end{array}$ \\
\hline & $\begin{array}{l}\text { Généralement effectué avec } \\
\text { un enquêteur, donc pas de } \\
\text { problème d'alphabétisation } \\
\text { des sujets }\end{array}$ & $\begin{array}{l}\text { Nécessité d'avoir des enquêteurs bien } \\
\text { formés (idéalement des diététiciens) } \\
\text { qui interrogent de façon neutre }\end{array}$ \\
\hline & Rapide à remplir ( \pm 30 min) & $\begin{array}{l}\text { Sous-estimation des ingérés (avec de } \\
\text { grandes variations selon les individus) }\end{array}$ \\
\hline & $\begin{array}{l}\text { Réalisation en face-à-face ou } \\
\text { par téléphone }\end{array}$ & \\
\hline \multirow{5}{*}{$\begin{array}{l}\text { Enregistrements } \\
\text { alimentaires } \\
\text { (méthode } \\
\text { prospective) }\end{array}$} & $\begin{array}{l}\text { Bonne idée de la diversité du } \\
\text { régime (d'autant plus que le } \\
\text { nombre de jours est élevé) }\end{array}$ & $\begin{array}{l}\text { Sujets devant être alphabétisés, sinon } \\
\text { nécessité d'avoir des enquêteurs, auquel } \\
\text { cas l'augmentation du coût est conséquente }\end{array}$ \\
\hline & $\begin{array}{l}\text { Méthode précise dans la } \\
\text { description des aliments, } \\
\text { les tailles des portions } \\
\text { (ou le poids, si les aliments } \\
\text { sont pesés) }\end{array}$ & Lourdeur de la méthode, chronophage \\
\hline & $\begin{array}{l}\text { Demande peu de moyens } \\
\text { logistiques }\end{array}$ & $\begin{array}{l}\text { Modification possible du comportement } \\
\text { (aliments et quantités) }\end{array}$ \\
\hline & & $\begin{array}{l}\text { La validité des données diminue avec } \\
\text { le nombre de jours }\end{array}$ \\
\hline & & Sous-estimation des ingérés \\
\hline
\end{tabular}


à des biais systématiques dans l'estimation des nutriments ingérés par les individus (Rutishauser, 2005). Leurs effets ne peuvent donc pas être réduits en augmentant le nombre d'observations.

\section{H Implications juridiques et éthiques}

Comme pour toutes recherches impliquant la participation d'êtres humains, les enquêtes visant à mesurer la consommation alimentaire tombent sous le coup de la déclaration d'Helsinki, datant de 1964. Cette dernière énonce des principes éthiques ayant pour but de protéger les participants de la recherche en termes de santé et bien-être, de protéger leur vie privée et d'assurer le maintien de leur intégrité.

Toute enquête ayant pour but de mesurer la consommation alimentaire doit faire l'objet d'une demande d'autorisation auprès d'un comité d'éthique local, lequel comité s'assurera du respect des principes éthiques. En outre, depuis 2018, en plus de l'autorisation d'un comité d'éthique, des démarches spécifiques en lien avec le règlement général sur la protection des données (RGPD) doivent être effectuées.

Dans certains contextes et selon les réalités socioculturelles, avant toute enquête auprès des populations, la prise de contact ou des demandes d'autorisation auprès des autorités locales et/ou religieuses peuvent également être une nécessité.

Avant toute enquête, chaque participant, ou chaque tuteur légal du participant si celui-ci est mineur, doit se voir expliquer les objectifs et implications de l'enquête, ce qu'il sera fait des données collectées et des résultats de l'enquête, mais également quels sont ses droits en tant que participant, notamment celui de refuser de participer, et ce afin de pouvoir donner son consentement éclairé.

De tout temps, l'alimentation a été régie et influencée par des codes culturels, sociaux ou religieux. Elle est soumise à un ensemble de normes pouvant porter par exemple sur des exclusions alimentaires ou des modes de préparation ou consommation des aliments. Lorsque l'on mesure la consommation alimentaire, il faut donc nécessairement prendre en compte ces spécificités afin de ne pas heurter la sensibilité des participants (Rucker et Rucker, 2016).

Selon la méthode utilisée et le contexte dans lequel elle est conduite, la mesure de la consommation alimentaire peut être plus ou moins intrusive. Au-delà même des questions posées aux participants sur leur alimentation - qui, selon les contextes et le niveau socio-économique des participants, peuvent être sensibles -, les enquêtes, essentiellement dans les pays du Sud du fait de taux d'alphabétisation parfois faibles, sont réalisées par des enquêteurs et idéalement au domicile des participants. Il faut donc porter une attention toute particulière à la formation des enquêteurs afin que ceux-ci adoptent une attitude neutre, respectueuse et culturellement adaptée.

\section{Mesures de la consommation alimentaire et collaborations entre disciplines}

Dans le contexte actuel des bouleversements alimentaires qui touchent tous les pays (par exemple, augmentation de la consommation d'aliments ultra-transformés, 
augmentation de la consommation de produits carnés), la mesure de la consommation alimentaire des individus permet de comprendre et d'évaluer l'impact de ces bouleversements, notamment sur le statut nutritionnel ou la santé des populations. En effet, c'est en mesurant la consommation alimentaire des individus que l'on peut par exemple savoir s'ils couvrent leurs besoins nutritionnels ou si certains modèles alimentaires constituent des facteurs de risque de développement de certaines maladies chroniques liées à l'alimentation comme l'hypertension ou le diabète de type 2 . Par ailleurs, la connaissance de la consommation alimentaire est indispensable pour concevoir et évaluer des politiques et outils d'intervention pertinents et adaptés, comme l'enrichissement de certains aliments en micronutriments pour lutter contre des carences (Eussen et al., 2015), ou encore la taxation de certains aliments néfastes à la santé pour en réduire la consommation (Alagiyawanna et al., 2015). Plus globalement, la mesure de la consommation alimentaire peut également permettre, à l'échelle d'une région ou d'un pays, de faire de la planification agricole afin qu'il y ait adéquation des disponibilités aux besoins de la population.

Généralement, lorsque l'on mesure la consommation alimentaire, un certain nombre de données sont recueillies de manière concomitante. Ainsi, il n'est pas rare que soient recueillis la provenance des aliments consommés, le lieu de consommation (chez soi, au travail, au restaurant...), ou encore les personnes avec qui les repas sont consommés. Ces données annexes peuvent avoir un intérêt pour d'autres disciplines telles que la sociologie ou l'économie. Inversement, la consommation alimentaire étant socialement et culturellement déterminée, les sciences sociales peuvent en amont permettre de prendre en compte certains facteurs afin que la mesure de la consommation alimentaire soit adaptée au contexte (par exemple, ne pas questionner sur des aliments tabous).

\section{" Un cas d'application du rappel des 24 heures}

Dans le cadre du présent chapitre, seule une des trois méthodes décrites précédemment sera illustrée, à savoir le rappel des 24 heures (méthode peu coûteuse pour la phase de collecte des données et fréquemment utilisée). La mesure de la consommation alimentaire via le rappel des 24 heures passe par trois étapes principales : une phase préparatoire, une phase de collecte et une phase de gestion des données. Il est à noter que ces trois phases sont communes aux autres méthodes de mesure de la consommation alimentaire avec toutefois des différences quant à leur contenu.

\section{Phase préparatoire}

L'opérationnalisation des différentes méthodes de mesure de la consommation alimentaire passe par la réalisation d'enquêtes nécessitant l'élaboration de plusieurs outils de collecte qui sont souvent fonction de l'objectif visé. Il s'agit notamment des questionnaires en eux-mêmes, des répertoires des aliments et recettes consommés par la population cible, des catalogues photos présentant différentes tailles de portion d'aliments, des mesures ménagères (bols, tasses, assiettes, cuillères...) utilisées pour la quantification des ingérés. Si l'objectif est de déterminer les apports nutritionnels ou le taux de couverture des besoins en nutriments, l'utilisation d'une 
table de composition ou d'une base de données sur la composition des aliments est indispensable. Il arrive qu'une base de données spécifique sur la composition des aliments soit pensée et développée lors de la phase préparatoire, bien qu'elle ne soit pas un outil de collecte, mais un outil de gestion de données.

Le questionnaire de rappel des 24 heures est adapté et élaboré selon le type d'étude et la cible. On peut choisir d'utiliser un guide d'entretien avec des questions ouvertes (il peut s'agir d'une feuille vierge sur laquelle sont recueillies les informations nécessaires) ou, à l'inverse, un questionnaire bien structuré avec des questions fermées.

Le répertoire des aliments (recettes incluses) renseigne sur le nom des aliments ou plats, généralement en plusieurs langues incluant des langues locales, leur identification à l'aide d'un code et les méthodes de mesures à utiliser pour les quantifier (prix, volume, poids, photo). C'est un outil dynamique, qui aide à l'identification et à la caractérisation des aliments/recettes durant l'enquête et qui peut être complété au cours de la collecte.

Le catalogue photos est souvent élaboré dans le but de faciliter l'estimation des quantités ingérées (figure 1.1). Il présente des photos standardisées de portions d'aliments ou recettes habituellement consommées par la population cible de l'étude. Il existe dans la littérature des directives indiquant comment développer et valider un tel outil (Nelson et al., 1998; Foster et al., 2005; Martin et al., 2014).

Il est parfois souhaitable de s'appuyer sur des mesures ménagères (généralement les plus couramment utilisées par et pour la population cible) afin d'estimer les quantités consommées. Ces ustensiles, gradués et calibrés, permettent au répondant d'indiquer le niveau correspondant à la quantité consommée d'aliment ou de plat.
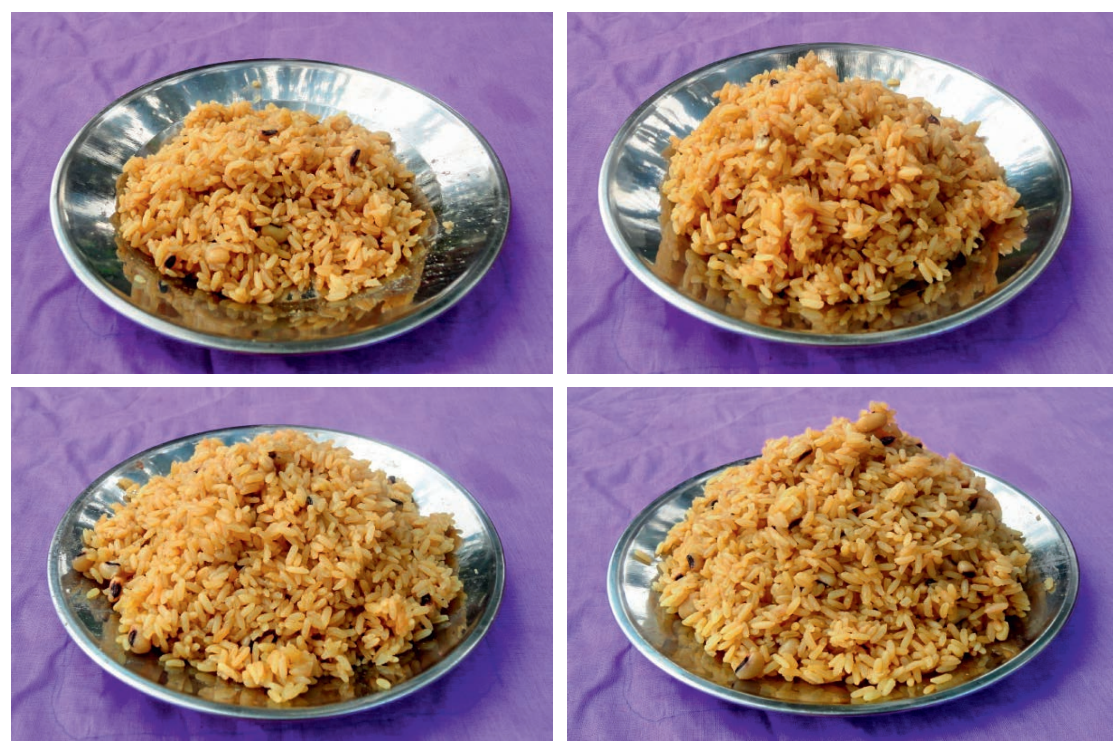

Figure 1.1. Exemple de photos de taille de portion utilisées au Bénin, extrait d'un catalogue de 35 aliments et recettes.

Source : Institut de recherche pour le développement (IRD) et faculté des Sciences agronomiques de l'université d'Abomey-Calavi (FSA/UAC), 2015. 


\section{Phase de collecte}

La phase de collecte de données concerne à la fois les données alimentaires en ellesmêmes, que l'on peut qualifier de données primaires (rappel des 24 heures dans le cas présent), ainsi que les données secondaires, par exemple un répertoire de recettes, ou encore des outils de quantification tels que les mesures ménagères ou le prix des aliments/plats. La collecte des données secondaires va dépendre des objectifs de l'enquête et de l'existence ou non de telles données pour la population cible.

\section{Données primaires}

Le principe de collecte des données grâce au rappel des 24 heures est décrit dans la section «Rappel des 24 heures».

Comme expliqué dans la méthode, le rappel des 24 heures est conduit idéalement au domicile des participants. Un des avantages de cette démarche réside dans le fait que, si un répondant n'arrive pas à quantifier ce qu'il a consommé avec les outils proposés, il peut utiliser ses propres ustensiles pour le faire. Par la suite, une correspondance est faite avec les outils de l'enquêteur.

Lors d'un rappel des 24 heures, on peut décider d'utiliser des recettes standards, représentant la composition moyenne pour cette recette, qui seront attribuées à tous les participants qui les auront consommées ou bien de recueillir pour chaque participant les recettes individuelles des plats qu'il aura mangés. La méthode «recette moyenne» représente un gain de temps lors de la phase de collecte des données, mais gomme les particularités individuelles dans la préparation des recettes. Il est à noter qu'un répertoire de recettes moyennes ne sera de toute façon jamais exhaustif et que, par conséquent, même lorsque l'on travaille avec les recettes moyennes, il reste possible de recueillir des recettes individuelles au cours de l'enquête.

\section{Données secondaires}

\section{Répertoire et composition de recettes moyenne}

Après avoir dressé la liste des recettes les plus couramment consommées (au cours de la phase préparatoire) par la population cible, on procède à l'observation desdites recettes. Lors de celle-ci, les différentes opérations unitaires qui constituent la recette sont suivies de bout en bout. Ainsi, tous les ingrédients entrant dans la recette (eau comprise) sont pesés avant et après préparation (épluchage, dénoyautage...). Le poids total de la préparation est ensuite mesuré avant et après cuisson. Lors de ces étapes, il est important de faire attention aux potentielles sources d'erreur qui pourraient affecter la qualité des données recueillies. Par exemple, il est nécessaire de toujours tarer la balance avant les prises de mesure, enlever les couvercles ou tout ustensile laissé dans les casseroles lors de la cuisson. Les observations doivent être répétées pour la même recette cuisinée successivement par des personnes différentes (idéalement 10 personnes). À l'issue des observations, les recettes sont «moyennées» pour obtenir une seule recette, dite «moyenne», qui sera affectée à tous les individus qui l'auront consommée. Une tâche intermédiaire importante est le choix des ingrédients à retenir pour la recette moyenne, qui doit être justifié et 
documenté. Parfois, le nombre ou le type d'ingrédient varie d'une personne à une autre. Par exemple, pour une même recette, certaines personnes peuvent utiliser la poudre de crevette pendant que d'autres emploient la farine de poisson, ou encore du gingembre et d'autres pas.

\section{Calibration des mesures ménagères}

Lors de l'enquête, on identifie les ustensiles couramment utilisés pour quantifier les différents aliments et recettes (figure 1.2). Pour chaque combinaison de mesure ménagère/aliment, il faut connaître la correspondance du poids de l'aliment/préparation en question. Pour cela, il faut calibrer les mesures ménagères. Par exemple, pour de la farine de maïs quantifiée à l'aide d'un bol équivalent à $350 \mathrm{ml}$ d'eau, la calibration consistera à remplir le bol en question de farine de maïs après avoir fait la tare, à peser le bol et la farine, à recommencer l'opération 10 fois afin de calculer un poids moyen.

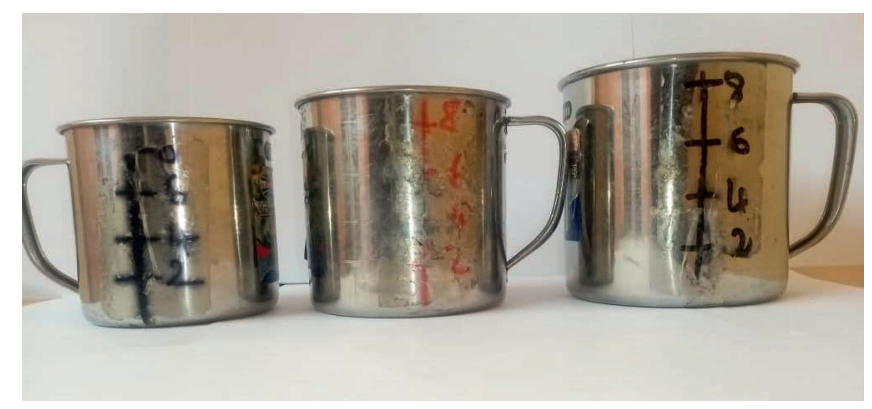

Figure 1.2. Exemple de mesures ménagères au Bénin dans le cadre du projet Recipal (Retard de croissance intra-utérin et paludisme).

Source : Institut de recherche pour le développement (IRD) et faculté des Sciences agronomiques de l'université d'Abomey-Calavi (FSA/UAC), 2015.

\section{Prix des aliments}

Pour chaque aliment quantifié en prix, il est nécessaire d'avoir une correspondance en poids. Pour cela, il faut réaliser une enquête «marché». À partir de la liste d'aliments et de recettes concernées, il faut échantillonner sur les marchés, dans les boutiques ou auprès des vendeurs de rue afin de connaître la quantité correspondante au prix payé par les participants. Toutefois, il faut tenir compte des variations saisonnières et spatiales des prix.

\section{Phase de gestion des données}

La première étape, déterminante pour la qualité des résultats, est la vérification et la correction de tous types de données saisies. Pour chaque donnée primaire ou secondaire, il faut en vérifier la plausibilité. Par exemple, lorsque des recettes ont été observées, il faut vérifier que le poids final de la recette après cuisson est inférieur au poids total des ingrédients avant cuisson. Ce type d'erreur peut s'expliquer par une mauvaise lecture du poids de la recette, une cuillère oubliée dans la casserole avant la pesée finale, un poids d'ingrédient mal noté... Dans ce cas, puisque chaque 
recette est observée plusieurs fois, il est possible de comparer les valeurs ou proportions des différentes observations pour prendre la décision de corriger, d'ajuster ou d'éliminer la donnée non plausible. Pour ce qui est des données primaires, la vérification de la plausibilité peut se faire sur les quantités d'aliments ou de plats consommés. Par exemple, il est peu probable qu'un individu consomme $4 \mathrm{~kg}$ de niébé sur une journée d'enquête.

Avant de passer au calcul des apports nutritionnels, il faut s'assurer que toutes les données relatives aux outils de quantification, ainsi que la table de composition des aliments sont finalisées. Lorsque, pour chaque individu, les ingérés en nutriments sont calculés sur une journée, il faut vérifier la plausibilité des apports énergétiques. Les enquêtes de consommation alimentaire sont sujettes à des problèmes de sur- et sous-déclaration, qui peuvent être identifiés grâce aux ingérés caloriques journaliers. Dans le cas du rappel des 24 heures, il s'agit essentiellement de sous-déclaration. Pour chaque sous-déclarant, il est essentiel de revenir aux données primaires pour vérifier ce qui a été déclaré par le répondant. Concernant les sous-déclarants, il existe, dans la littérature, différentes façons de les traiter (Ferrari et al. 2002; Gibson et al. 2017).

\section{W Adaptation de la méthode au contexte d'application}

Les méthodes de mesure de la consommation alimentaire doivent être adaptées au contexte de leur mise en œuvre, à savoir la population cible, le milieu (urbain ou rural), les normes socioculturelles, les moyens matériels, humains et financiers disponibles, le temps dont on dispose pour faire l'enquête. Par exemple, selon que la population cible est alphabétisée ou non, il faudra adapter le mode de recueil des données, c'est-à-dire le questionnaire, afin qu'il soit auto-administré ou administré par un enquêteur.

Les outils de quantification, que ce soit les prix, les mesures ménagères ou le catalogue photos, ne sont valides que pour la population et le contexte pour lesquels ils ont été développés et validés. Il en est de même pour le questionnaire de fréquence de consommation alimentaire.

Lorsque l'on mesure la consommation alimentaire, il est recommandé de ne pas influer sur le comportement alimentaire des participants. Cependant, dans le cas où les individus se nourrissent à partir d'un plat commun, on peut adapter la méthode et alors demander aux participants de préparer des assiettes individuelles de manière à mieux estimer les quantités consommées. Cela est envisageable sur une courte période de temps allant d'un à trois jours. Cette adaptation n'est pas envisageable quand il s'agit d'une étude sur la fréquence de consommation alimentaire. Dans le cas où l'on veut effectuer une enquête de consommation alimentaire dont l'objectif est de capturer les habitudes alimentaires d'une population cible, l'utilisation du questionnaire de fréquence est la méthode la plus adaptée. Pour cela, il faut qu'il existe déjà un questionnaire de fréquence développé et validé pour la population d'intérêt. Si ce n'est pas le cas, il est possible de s'orienter vers une autre méthode telle que le rappel des 24 heures ou l'enregistrement alimentaire en augmentant la durée du recueil. 
Le choix de la méthode la plus adéquate pour recueillir des données de consommation alimentaire va dépendre non seulement des objectifs de l'étude, mais également de la population concernée par l'étude, des outils existants ou non, ainsi que des ressources humaines et financières disponibles. La collecte de données de consommation alimentaire peut paraitre relativement simple (au moins pour les trois méthodes concernées par ce chapitre). Cependant, il faut avoir à l'esprit que les phases de préparation de la collecte et de gestion des données recueillies sont, quant à elles, généralement complexes et chronophages, et souvent sous estimées.

\section{" Références}

Alagiyawanna A., Townsend N., Mytton O., Scarborough P., Roberts N., Rayner M., 2015. Studying the consumption and health outcomes of fiscal interventions (taxes and subsidies) on food and beverages in countries of different income classifications; a systematic review. BMC Public Health, 15 (887). https://doi.org/10.1186/s12889-015-2201-8

Arsenault J. E., Moursi M., Olney D. K., Becquey E., Ganaba R., 2020. Validation of 24-h dietary recall for estimating nutrient intakes and adequacy in adolescents in Burkina Faso. Maternal and Child Nutrition, 16 (4), e13014. https://doi.org/10.1111/mcn.13014

Beaton G. H., 1994. Approaches to analysis of dietary data: relationship between planned analyses and choice of methodology. American Journal of Clinical Nutrition, 59 (1), 253S-261S. https://doi.org/10.1093/ajcn/59.1.253S

Benassar B., Goy J., 1975. Contribution à l'histoire de la consommation alimentaire du XIV au XIX ${ }^{\mathrm{e}}$ siècle. Annales. Économies, sociétés, civilisations, 30 (2-3), 402-430. https://doi.org/10.3406/ahess.1975.293615

Biro G., Hulshof K. F., Ovesen L., Amorim Cruz J. A., 2002. Selection of methodology to assess food intake. European Journal of Clinical Nutrition, 56 (2), S25-S32. https://doi.org/10.1038/sj.ejcn.1601426 Cade J., Thompson R., Burley V., Warm D., 2002. Development, validation and utilisation of food frequency questionnaires - a review. Public Health Nutrition, 5 (4), 567-587.

https://doi.org/10.1079/PHN2001318

Church S. M., 2006. The history of food composition databases. Nutrition Bulletin, 31 (1), 15-20. https://doi.org/10.1111/j.1467-3010.2006.00538

Eussen S., Alles M., Uijterschout L., Brus F., van der Horst-Graat J., 2015. Iron intake and status of children aged 6-36 months in Europe: a systematic review. Annals of Nutrition and Metabolism, 66 (2-3), 80-92. https://doi.org/10.1159/000371357

Ferrari P., Slimani N., Ciampi A., Trichopoulou A., Naska A., Lauria C., Veglia F., Buenode-Mesquita H. B., Ocké M. C., Brustad M., Braaten T., Tormo M. J., Amiano P., Mattisson I., Johansson G., Welch A., Davey G., Overvad K., Tjønneland A., Clavel-Chapelon F., Thiebaut A., Linseisen J. H., Boeing H., Hemon B., Riboli E., 2002. Evaluation of under- and overreporting of energy intake in the 24-hour diet recalls in the European Prospective Investigation into Cancer and Nutrition (EPIC). Public Health Nutrition, 5 (6b), 1329-45. https://doi.org/10.1079/PHN2002409

Flandrin J.-L., 1983. La diversité des goûts et des pratiques alimentaires en Europe du $\mathrm{XVI}^{\mathrm{e}}$ au XVIII $^{\mathrm{e}}$ siècle. Revue d'Histoire Moderne \& Contemporaine, 30 (1), 66-83. [En ligne] https://www.cairn. info/revue-d-histoire-moderne-et-contemporaine-1983-1-page-66.htm (consulté le 16/04/2018).

Foster E., Matthews J. N., Nelson M., Harris J. M, Mathers J. C., Adamson A. J., 2005. Accuracy of estimates of food portion size using food photographs - the importance of using age-appropriate tools. Public Health Nutrition, 9 (4), 509-514. https://doi.org/10.1079/PHN2005872

Gibson R. S., Charrondiere U. R., Bell W., 2017. Measurement Errors in Dietary Assessment Using Self-Reported 24-Hour Recalls in Low-Income Countries and Strategies for Their Prevention. Advances in Nutrition, 8 (6), 980-991. https://doi.org/10.3945/an.117.016980 
Helsing E., 1991. Use of a nutrition information system, in : Food and Health Data. Their Use in Nutrition Policy-Making, Becker W., Helsing E. (éd.), Copenhagen, World Health Organization, 15-24 (coll. WHO Regional Publications, European Series, 34).

Martin C. K., Nicklas T., Gunturk B., Correa J. B., Allen H. R., Champagne C., 2014. Measuring food intake with digital photography. Journal of Human Nutrition and Diet, 27 (1), $72-81$.

https://doi.org/10.1111/jhn.12014

Nelson M., Haraldsdottir J., 1998. Food photographs: practical guidelines 1. Design and analysis of studies to validate portion size estimates. Public Health Nutrition, 1 (4), 219-203.

https://doi.org/10.1079/phn19980038

Rucker B. R, Rucker M. R., 2016. Nutrition: ethical issues and challenges. Nutrition Research, 36 (11), 1183-1192. https://doi.org/10.1016/j.nutres.2016.10.006

Rutishauser I. H. E., 2005. Dietary intake measurements. Public Health Nutrition, 8 (7a), 11001107. https://doi.org/10.1079/phn2005798

Thompson F. E., Byers T., 1994. Dietary assessment resource manual. The Journal of Nutrition, 124 (11), 2245S-2317S. https://doi.org/10.1093/jn/124.suppl_11.2245s

Webster-Gandy J., Madden A., Holdsworth M., 2020. Nutrition assessment, in Webster-Gandy J., Madden A., Holdsworth M. (éd.), Oxford handbook of nutrition and dietetics, $3^{\text {rd }}$ edition, Oxford, Oxford University Press, 37-60. https://doi.org/10.1093/med/9780199585823.003.0004 

Chapitre 2

\title{
La mesure de la sécurité alimentaire des individus et des ménages : potentiel et défis de la collaboration entre sciences de la nutrition et sciences sociales
}

\author{
EMMANUELLE BougueT ${ }^{a}$, AlisSia LOURMe-RUIZ ${ }^{a}$ et ANNE BICHARD ${ }^{b}$ \\ a CIRAD, UMR MoISA, F-34398 Montpellier, France; MoISA, Université Montpellier, CIHEAM-IAMM, \\ CIRAD, INRAE, Institut Agro, IRD, Montpellier, France. \\ ${ }^{\mathrm{b}}$ IRAM, Institut de recherche et d'application des méthodes de développement, France.
}

Deux grands types d'indicateurs sont utilisés pour mesurer le degré de sécurité alimentaire des individus et des ménages : ceux qui s'intéressent aux expériences vécues par les mangeurs et à leur propre évaluation de leur situation; ceux qui s'appuient sur une mesure de leur consommation alimentaire. Le score de diversité alimentaire appartient à cette deuxième catégorie. Il rend compte de la qualité nutritionnelle de l'alimentation des individus et peut être utilisé avec d'autant plus de pertinence qu'il s'accompagne d'approches plus compréhensives des pratiques alimentaires et des points de vue des mangeurs.

«La sécurité alimentaire existe lorsque tous les êtres humains ont, à tout moment, un accès physique et économique à une nourriture suffisante, saine et nutritive leur permettant de satisfaire leurs besoins énergétiques et leurs préférences alimentaires pour mener une vie saine et active». Cette définition issue du Sommet mondial de l'alimentation de 1996 s'est largement imposée chez les acteurs du développement (ONG, services gouvernementaux, institutions internationales) comme chez les chercheurs (nutritionnistes, épidémiologistes, économistes, sociologues, anthropologues, etc.). Elle pose cependant des problèmes considérables d'observabilité et de mesure. Le concept recouvre en effet des dimensions multiples (sanitaire, économique, sociale et culturelle), et il se décline à différentes échelles (des individus jusqu'aux pays en passant par les ménages). L'enjeu pour des indicateurs de sécurité alimentaire, comme pour tout indicateur composite d'une réalité complexe, est de mesurer une variable non directement observable en mobilisant des approximations (ou proxys) qui satisfassent des critères minimums de fiabilité, de pertinence et d'utilité pour l'action et pour la recherche. 
Ce chapitre propose un panorama sélectif des principaux indicateurs de la sécurité alimentaire des individus et des ménages, depuis une perspective de non-nutritionnistes ${ }^{3}$. La première section revient sur l'historique de construction de deux grandes familles d'indicateurs, axées l'une sur l'expérience d'insécurité alimentaire, l'autre sur la consommation. La deuxième section en précise les champs d'application. La troisième section s'intéresse aux aspects pratiques et relationnels de la production de ces indicateurs sur le terrain. La quatrième section propose une réflexion sur la contribution des indicateurs au dialogue entre disciplines. La cinquième section présente un retour d'expérience de la mise en œuvre d'un indicateur de qualité nutritionnelle du régime dans le cadre d'un projet de recherche sur les liens entre agriculture et alimentation au Burkina Faso : le score de diversité alimentaire individuel (Women Dietary Diversity Score, WDDS-10), basé sur le comptage de 10 groupes d'aliments consommés 4 .

\section{" Origine des indicateurs de sécurité alimentaire}

Les principaux indicateurs de sécurité alimentaire trouvent leur origine dans le monde des organismes multilatéraux $\left(\mathrm{FAO}, \mathrm{PAM}^{5}\right)$ et de l'aide internationale (l'agence américaine d'aide au développement USAID et l'ONG Care), avec l'appui méthodologique d'universitaires ${ }^{6}$. L'histoire de ces indicateurs reflète l'évolution du concept de sécurité alimentaire. La (mal)nutrition a longtemps été le domaine des épidémiologistes et des nutritionnistes. Leur objet d'étude est centré sur l'état nutritionnel des individus, résultant de l'interaction entre alimentation et santé. La notion de sécurité alimentaire est plus globale : ses différents piliers (disponibilité, accès, utilisation et stabilité) resituent l'état nutritionnel des individus dans un contexte social et économique plus large (l'échelle du ménage prend ici tout son sens), et renvoient à d'autres domaines de connaissance que la nutrition ou l'épidémiologie (FAO et OMS, 1992).

Le concept de sécurité alimentaire a par ailleurs évolué en parallèle à celui de malnutrition. Les nutritionnistes ont longtemps mis l'accent sur les carences en calories ou en protéines, directement liées à des pénuries en aliments de base (estimées par les disponibilités céréalières ou les enquêtes budget-consommation). Depuis la fin des années 2000, une attention croissante est portée aux carences en micronutriments, qui renvoient davantage à la qualité de l'alimentation. Lémergence des indicateurs de diversité alimentaire reflète cette évolution, qui se traduit par un glissement de l'expression «sécurité alimentaire» vers celle de «sécurité alimentaire et nutrition-

3. Les auteures de ce chapitre s'inscrivent dans le champ des sciences sociales appliquées aux questions de développement et ont une longue expérience de collaboration avec des nutritionnistes.

4. Groupe 1 : céréales, racines et tubercules blancs; groupe 2 : pois et haricots; groupe 3 : noix et graines; groupe 4 : produits laitiers; groupe 5 : viandes, volailles et poissons; groupe $6: œ u f s ;$ groupe 7 : légumes feuilles riches en vitamine $\mathrm{A}$; groupe 8 : fruits et légumes riches en vitamine $\mathrm{A}$; groupe 9 : autres légumes; groupe 10 : autres fruits.

5. La FAO (Organisation des Nations unies pour l'alimentation et l'agriculture) et le PAM (Programme alimentaire mondial), sont deux organismes des Nations unies.

6. Le projet FANTA (Food and Nutrition Assistance Project, https://www.fantaproject.org/ [dernière consultation le 27/08/2021]) est un bon exemple de collaboration entre agences internationales (USAID, FAO) et universités (Cornell, Tufts) pour le développement et la validation d'indicateurs. Luniversité Tufts propose un recensement très complet des indicateurs de sécurité alimentaire : https://inddex.nutrition.tufts.edu/data4diets/indicators (dernière consultation le 27/08/2021). 
nelle», ou SAN. Plus récemment, la malnutrition par excès (en graisse, sucre, sel), qui émerge dans des populations par ailleurs exposées à l'insécurité alimentaire, vient complexifier le concept de SAN.

On peut distinguer deux grandes familles d'indicateurs selon l'angle d'approche de la SAN (encadré 2.1 et tableau 2.1).

La première famille (HFIAS, HHS, FIES, CSI $^{7}$ ) s'intéresse à l'expérience de l'insécurité alimentaire et aux stratégies mises en œuvre par les ménages pour s'en prémunir ou y faire face. Elle est associée à des acteurs de l'aide sociale ${ }^{8}$ ou de l'urgence humanitaire (PAM).

La deuxième famille (HDDS, WDDS, $\mathrm{MDD}-\mathrm{W}, \mathrm{FCS}^{9}$ ) renvoie à la consommation alimentaire, abordée à partir de scores de diversité. Elle est plutôt associée à des acteurs de la nutrition ou de la sécurité alimentaire (projet FANTA).

Selon les cas, ces indicateurs se déclinent au niveau individuel ou celui du ménage (généralement défini comme une unité de résidence et de consommation), car c'est à ce niveau plus agrégé que se prennent les décisions en lien avec les pratiques alimentaires

\section{Encadré 2.1 : Différences d'approche par famille d'indicateurs}

Famille 1 : expérience et ressenti de l'insécurité alimentaire (cas du HFIAS)

\begin{tabular}{cl}
\hline Domaine & \multicolumn{1}{c}{ Exemples de questions } \\
\hline Anxiété & Étiez-vous préoccupé du fait que votre ménage n'avait pas assez de nourriture? \\
\hline Qualité & $\begin{array}{l}\text { Est-ce que vous-même ou un membre de votre ménage n'avez pas pu manger } \\
\text { les types de nourriture que vous préférez à cause d'un manque de ressources? }\end{array}$ \\
\hline Quantité & $\begin{array}{l}\text { Est-ce que vous-même ou un membre de votre ménage avez mangé un repas } \\
\text { plus petit que vous n'auriez souhaité parce qu'il n'y avait pas assez à manger? }\end{array}$ \\
\hline Faim & $\begin{array}{l}\text { Est-ce que vous-même ou un membre de votre ménage avez passé toute } \\
\text { une journée et toute une nuit sans manger parce qu'il n'y avait pas assez } \\
\text { de nourriture? }\end{array}$ \\
\hline
\end{tabular}

Source : extrait de Coates et al. 2007, p. 4

Famille 2 : consommation - rappel qualitatif des 24 heures pour les scores de diversité alimentaire (cas du WDDS/MDD-W)

«Veuillez indiquer ce que vous avez mangé et bu hier (repas et collations), que ce soit pendant la journée ou la nuit... Commencez par le premier aliment ou la première boisson consommé(e) le matin $[\ldots]$ ».

Source : Kennedy et al. 2012, p. 7

7. Dans ce chapitre, nous utilisons les acronymes des indicateurs dans leur version anglaise. HFIAS : échelle de l'accès déterminant l'insécurité alimentaire des ménages. HHS : échelle de la faim des ménages, extrait du HFIAS; FIES : échelle de mesure de l'insécurité alimentaire vécue; CSI : index de stratégie de gestion de la vulnérabilité.

8. À l'origine, l'HFIAS a été développé pour évaluer des programmes d'aide alimentaire auprès de ménages pauvres aux États-Unis. L'indicateur a ensuite été adapté et étendu aux contextes de pays du Sud. Actuellement, il tend à être remplacé par le FIES.

9. HDDS : score de diversité alimentaire des ménages; WDDS : score de diversité alimentaire des femmes; MDD-W : diversité alimentaire minimale des femmes; FCS : score de consommation alimentaire. 
et de soin, qui vont in fine contribuer à l'état nutritionnel des individus qui le composent. Nous reviendrons sur cette importante distinction dans les sections suivantes.

Ces indicateurs ont été construits à partir d'un jeu de critères communs, qui renvoient à la fois à des considérations méthodologiques et opérationnelles :

- pertinence : l'indicateur doit capter différentes dimensions du réel en rapport avec la SAN des individus et des ménages. Pour les épidémiologistes qui réfléchissent en termes de diagnostic et de prévalence, ainsi que pour les économistes quantitatifs, la validation de la pertinence consiste à estimer les niveaux de corrélation avec d'autres mesures éprouvées de la malnutrition et de l'insécurité alimentaire (Hoddinott et Yohannes 2002; Leroy et al., 2015). Pour les économistes ou socio-économistes qui réfléchissent plutôt en termes de moyens d'existence et de stratégies, la validation peut être plus qualitative, mais suit la même approche;

- sensibilité aux variations, pour pouvoir se prêter à des diagnostics, des comparaisons, et plus généralement à des analyses statistiques;

- comparabilité dans le temps, dans l'espace ou entre sous-populations, ce qui implique un travail important de standardisation des questions, des réponses, des analyses;

- simplicité (relative) de mise en œuvre. Les indicateurs de SAN opèrent un arbitrage en faveur d'informations moins précises, mais plus faciles et rapides à collecter que d'autres méthodes. Pour les nutritionnistes et épidémiologistes, l'idéal est de procéder à des mesures quantitatives de consommation alimentaire, complétées par des mesures anthropométriques ou des analyses de sang, mais ces dispositifs sont très coûteux et intrusifs pour les populations. Pour les économistes, la simplification se fait en référence aux enquêtes budget-consommation assurées par les instituts nationaux de statistiques, également très lourdes et qui comportent d'importantes erreurs de mesure;

- simplicité de manipulation. Les indicateurs se présentent comme des scores agrégeant différentes informations; ils peuvent se prêter à des statistiques descriptives (par exemple pour des tableaux de bord) ou être inclus dans des modèles statistiques ou économétriques plus sophistiqués;

- cette simplification de la méthode de recueil et de traitement des indicateurs n'est pas uniquement d'ordre méthodologique. Elle a été pensée pour pouvoir déléguer et démultiplier la production de données. Des manuels détaillés (FAO, s.d.; Coates et al., 2007; Maxwell et Caldwell, 2008; World Food Program, 2008; Ballard et al., 2011; Kennedy et al., 2012; FAO, 2021) ont été rédigés dans l'objectif de faciliter l'appropriation des méthodes par des acteurs de terrain ou des chercheurs de différentes disciplines.

\section{H Utilisation des indicateurs}

\section{Des scores pour qualifier la SAN des individus ou des ménages}

Les indicateurs se présentent comme un score agrégeant des ressentis, des expériences ou des stratégies dans le cas du HFIAS, HHS, FIES et CSI, des groupes d'aliments dans le cas des scores de diversité (HDDS, WDDS, IDDS, FCS), avec un système de codage commun en termes d'occurrences (éventuellement combinées à des fréquences) sur une période de rappel donnée. 


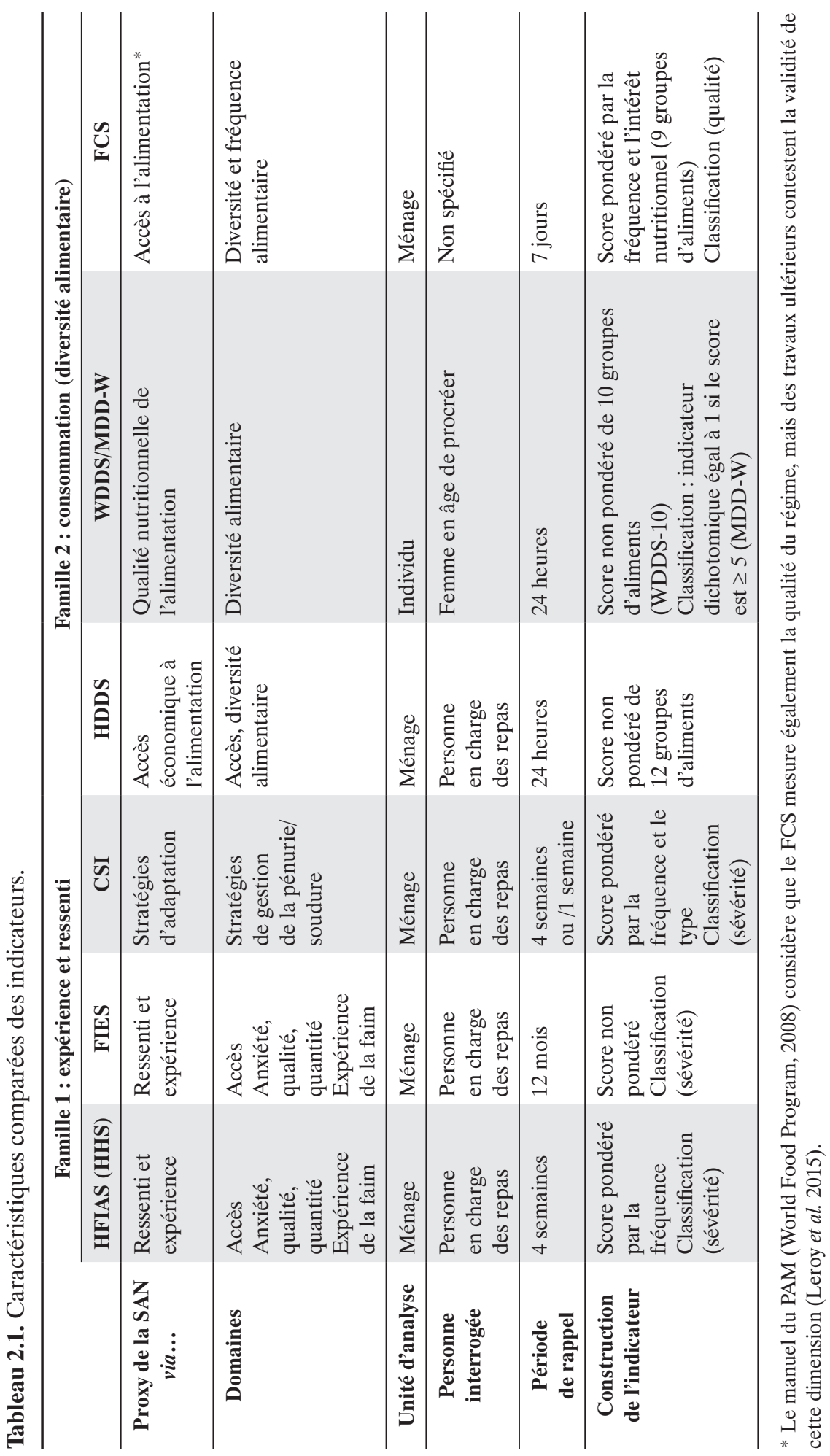


Le CSI incorpore un système de pondération, réfléchi au cas par cas en fonction de la connaissance préalable du contexte (éventuellement en concertation avec les parties prenantes). Ce système présente l'avantage de mieux refléter les enjeux des situations locales, mais il limite la possibilité de comparer différents contextes.

Les méthodes de construction des scores de diversité alimentaire s'ajustent différemment à l'évolution du concept de SAN. Le WDDS/MDD-W, seul indicateur validé évaluant la qualité nutritionnelle de l'alimentation individuelle, inclut dix groupes alimentaires présentant un intérêt nutritionnel, sans pondération. Le HDDS, construit à partir de 12 groupes non pondérés, a été validé comme indicateur d'un meilleur accès économique du ménage à l'alimentation. Cependant, trois de ces groupes (sucreries, huiles et graisses, et boissons) sont désormais associés à la malnutrition par excès, et leur prise en compte peut être considérée comme contradictoire. Le FCS utilise des poids variant de 0 à 4 pour tenir compte du niveau de qualité nutritionnelle assigné à chaque groupe, mais il n'a pas été validé statistiquement.

\section{Interprétation et comparaison}

De par leur caractère agrégé, les valeurs prises par les indicateurs sont difficiles à interpréter isolément. Des seuils sont proposés pour le HFIAS et le CSI (niveaux de gravité de l'insécurité alimentaire), le MDD-W (avec un score moyen de 5 groupes ou plus, les femmes ont plus de chances de couvrir leurs besoins en micronutriments), ainsi que le FCS (niveaux de qualité du régime alimentaire). Dans tous les cas, les manuels insistent sur les précautions à prendre dans le maniement et l'interprétation de ces seuils et des classifications qui en résultent. Par ailleurs, quel que soit l'indicateur, il est toujours possible de revenir aux données sources pour affiner les analyses : le ressenti et les pratiques du HFIAS, les stratégies du CSI, les groupes alimentaires qui contribuent aux scores de diversité.

Il est plus facile d'interpréter les variations d'un indicateur donné dans le temps, en termes d'amélioration ou de détérioration. Cela suppose néanmoins de faire l'hypothèse que la période de rappel est représentative d'une situation «typique». Une vigilance particulière s'impose pour les variations saisonnières, très marquées en zones rurales et agricoles. Par exemple, une augmentation du score de diversité alimentaire, liée à la disponibilité ponctuelle de fruits et légumes, peut aller de pair avec une détérioration des indicateurs de ressenti de SAN, liée à la réduction des stocks de céréales (Hoddinott et Yohannes, 2002; Savy et al., 2006; Lourme-Ruiz et al., 2021). D'autre part, pour suivre des évolutions structurelles, les mesures doivent être répétées à la même saison.

La comparaison entre populations soulève des difficultés spécifiques, particulièrement pour les indicateurs liés à l'expérience et aux stratégies associées à l'insécurité alimentaire. Même si les questions sont standardisées, les réponses dépendent du contexte socio-culturel, ce qui peut invalider la comparaison. Par exemple, des populations régulièrement exposées à l'insécurité alimentaire peuvent manifester une forme de résignation et donc exprimer moins d'anxiété que d'autres pourtant moins exposées. C'est ce constat qui a amené à proposer le HHS, centré sur un sous-groupe de questions relatives à l'expérience de la faim, considérée comme plus universelle et présentant un meilleur potentiel de comparabilité que le HFIAS ou le FIES. Cependant, le gain en comparabilité se fait au prix d'une perte en spécificité dans les degrés et les différences de manifestation de l'insécurité alimentaire. 
Dans la mesure où le découpage en groupes alimentaires conserve en grande partie sa pertinence d'un contexte à un autre, les indicateurs de diversité alimentaire sont plus adaptés à des comparaisons entre régions. Cependant, un même score peut masquer de grandes disparités de qualité nutritionnelle.

Une attention particulière doit être portée à la différence entre MDD-W/WDDS et HDDS. En effet, celle-ci ne peut pas être assimilée à un changement d'échelle d'analyse, de l'individu vers le ménage. Les deux indicateurs mesurent des dimensions différentes de la sécurité alimentaire : qualité nutritionnelle du régime pour les premiers, accès économique à l'alimentation pour le second. Par ailleurs, à la différence des indicateurs individuels, le HDDS ne mesure pas la consommation hors-foyer (à la cantine, au marché, etc.), qui peut induire des disparités de consommation entre les membres d'un même ménage. Enfin, le MDD-W, bien que renseigné à l'échelle de l'individu, n'est validé comme indicateur de qualité nutritionnelle qu'à l'échelle d'une population.

\section{Questions opérationnelles, questions de recherche}

Parce qu'ils ont été développés par des organismes de l'aide alimentaire et du développement, les indicateurs de SAN répondent en premier lieu à des besoins opérationnels : estimer des niveaux de prévalence et des degrés de sévérité de l'insécurité alimentaire; déclencher, cibler et paramétrer des interventions d'assistance alimentaire ou nutritionnelle, éventuellement dans l'urgence; réaliser du suivi-évaluation ou des études d'impact. Ces indicateurs sont également utilisés dans le cadre de projets de développement plus larges ou de plus long terme (en agriculture ou éducation notamment), affichant une démarche «sensible à la nutrition» (Ruel et Alderman, 2013). Les scores de diversité alimentaire sont promus par des bailleurs tels que l'Union européenne, qui incitent les opérateurs des projets à les utiliser dans leur cadre de résultats.

Ces indicateurs peuvent également répondre à des questions de recherche. Les manuels s'avèrent utiles pour les chercheurs de toutes disciplines, car, au-delà de l'exposé du protocole et des conseils pratiques pour le travail de terrain, ils intègrent des considérations épistémologiques et méthodologiques sur les indicateurs eux-mêmes (unités d'analyse, validation statistique, qualité de la mesure), et de méta-analyse pour la dimension comparative. Les travaux de recherche mobilisant ces indicateurs peuvent s'inscrire dans des études nutritionnelles ou dans des études plus larges sur le fonctionnement socio-économique des ménages, sur les liens entre agriculture et alimentation, etc. (voir plus loin dans ce chapitre la section 5 «Étude de cas : diversité alimentaire des ménages agricoles au Burkina Faso»).

\section{- Passation des questionnaires: questions déontologiques et pratiques}

La passation des questionnaires pour la construction des indicateurs soulève des questions déontologiques et pratiques. Sur le plan déontologique (et parfois réglementaire), le caractère personnel des données suppose de mobiliser des dispositifs de protection, tels que l'approbation par un comité d'éthique, le consentement éclairé des participants et l'anonymisation des bases. La durée de passation du 
questionnaire, qui représente un coût d'opportunité pour les enquêtés, est également un paramètre à prendre en compte. Sur le plan pratique, la qualité des données recueillies est tributaire des biais inhérents au recueil de données déclaratives : biais cognitifs, de lassitude, ou de désirabilité sociale. Ces considérations générales prennent un relief particulier en raison du caractère sensible de l'insécurité alimentaire. Les enquêteurs doivent composer entre la rigueur méthodologique requise pour obtenir des données standardisées et l'empathie nécessaire pour instaurer un climat de confiance, parfois dans des situations de tensions, voire de détresse.

Les questionnaires HFIAS et le CSI sont rapides à passer, et peu sensibles aux biais cognitifs : ils ne posent pas de problèmes de compréhension ou de mémoire sur la période de rappel, même si les questions de fréquence peuvent être plus difficiles à renseigner que les questions sur l'occurrence. En revanche, certaines questions sont sensibles, notamment celles concernant l'expérience de la faim ou les stratégies d'évitement. Le risque de biais de désirabilité sociale est élevé, et peut aller dans les deux sens : sur-déclaration de l'insécurité alimentaire si les enquêtés anticipent de bénéficier d'un futur programme; sous-déclaration si certaines pratiques sont stigmatisées socialement.

Pour les scores de diversité, le risque de biais de désirabilité sociale est également présent, car des représentations normatives, variables selon les contextes, peuvent être associées à certains aliments. Pour le HDDS et le WDDS/MDD-W, le rappel des 24 heures (chapitre 1) sollicite relativement peu la mémoire des enquêtés, mais la méthodologie implique de relever le détail des ingrédients des plats consommés, ce qui augmente le risque de lassitude, le biais de mémoire, et le biais cognitif en cas de plats préparés par un tiers. La période de rappel du FCS est plus longue (7 jours), mais l'effort de mémoire supplémentaire est compensé par une entrée par les groupes d'aliments, plus globale que les plats et les ingrédients.

Enfin, une attention particulière doit être portée aux répondants des questionnaires. En fonction du contexte et du statut, les personnes interrogées peuvent être peu habituées à prendre la parole; inversement, dans des contextes de forte intervention, elles peuvent devenir des «enquêtées professionnelles». Pour les indicateurs recueillis au niveau du ménage, le risque de biais cognitifs ou de désirabilité sociale est d'autant plus grand que l'on demande à la personne interrogée, généralement la femme en charge des repas, de s'exprimer au nom de son ménage, c'est-à-dire d'un groupe plus ou moins étendu (dont il faut préciser les contours), avec des variations plus ou moins grandes entre ses membres.

\section{W Apports de la méthode pour les disciplines connexes}

Dans cette section, nous nous intéressons au potentiel de synergies entre les sciences de la nutrition et les sciences sociales, pour éclairer les mécanismes qui caractérisent ou qui déterminent une situation d'insécurité alimentaire, au niveau des ménages ou des individus qui les composent.

Qu'on se place dans une perspective de développement ou de recherche, les indicateurs de SAN peuvent être resitués dans des champs plus larges : pauvreté, vulnérabilité, moyens d'existence, inégalités intra-ménage, perspective de genre, etc. 
Le caractère normé des indicateurs de SAN devrait a priori faciliter le dialogue entre opérateurs et chercheurs, ou entre chercheurs de différentes disciplines.

On observe de fait un nombre croissant de travaux croisant indicateurs de diversité alimentaire et différentes variables agricoles (Jones, 2017; Sibathu et Qaim, 2018). Cependant, les conditions d'un dialogue interdisciplinaire fécond ne sont pas toujours réunies. Des débats ont émergé entre économistes agricoles et nutritionnistes, qui relèvent des emprunts sélectifs, voire des confusions méthodologiques ou conceptuelles (sur les unités d'analyses, les groupes, etc.), contestent dès lors la validité de certaines interprétations, et préconisent a minima de ne pas se réclamer de l'indicateur «officiel» lorsque la méthode d'enquête et d'analyse dévie par rapport aux manuels standardisés (Verger et al., 2019). Inversement, les sciences sociales peuvent reprocher aux nutritionnistes une vision trop étroite de l'alimentation (réduite à ses composantes nutritionnelles), des individus (réduits à leur état de santé) et plus encore de leur environnement, qu'il soit familial, social, culturel, matériel.

Le potentiel de synergie tient également à la question des articulations entre unités d'analyse. Le ménage est un échelon clé, mais le changement d'échelle de la SAN de l'individu vers celle du ménage ne va pas de soi. Les contours du ménage eux-mêmes peuvent être mouvants, selon qu'on le considère comme une unité de résidence, de revenus, de consommation, ou plus spécifiquement de consommation alimentaire. La question des contours se pose d'autant plus dans des contextes de familles élargies, polygames ou intergénérationnelles. Le cas des ménages agricoles, à la fois producteurs et consommateurs d'aliments, ajoute encore de la complexité. C'est dans la caractérisation des contours et des interactions au sein du ménage que les sciences sociales (économie, socio-anthropologie) peuvent apporter des éclairages pour mieux comprendre comment se définissent des stratégies, comment s'opèrent les arbitrages pour générer, contrôler et répartir des ressources, et quelle place occupe l'alimentation dans ces stratégies et ces arbitrages.

Le dialogue entre disciplines pourrait enfin être enrichi via une analyse des processus nourrie par des approches qualitatives (ethnographie). En effet, les indicateurs relevés au niveau des ménages (HFIAS, HDDS, FCS, CSI) ne disent rien des processus de décision et d'allocation impliquant des individus ou sous-groupes au sein du ménage (conjoints, parents/enfants, etc.), sur la nature des relations qui sous-tendent ces processus (coopération, subordination, négociation, compromis, conflit), et sur les éventuelles inégalités (de genre, de génération) qui en découlent. Les scores individuels (WDDS/MDD-W et ses adaptations pour d'autres individus) peuvent identifier des différences interindividuelles au sein d'un ménage, mais n'ont pas non plus vocation à décrire les mécanismes sous-jacents.

\section{"Étude de cas : diversité alimentaire des ménages agricoles au Burkina Faso}

Cette section s'appuie sur le cas du projet $\operatorname{Relax}^{10}$, un projet pluridisciplinaire (nutrition, économie, agronomie, socio-anthropologie, géographie, sciences politiques et

10. https://relax.cirad.fr (dernière consultation le 27/08/2021). 
modélisation) visant à explorer le nexus «agriculture/nature/marché/alimentation» dans une province de l'ouest du Burkina Faso. Un score de diversité alimentaire individuel (WDDS-10 et MDD-W) a été intégré dans une enquête économique menée auprès de 300 ménages agricoles. Cet exemple illustre le décalage entre la simplicité apparente du manuel et la mise en ouvre effective : la construction d'un score de diversité est une opération plus complexe qu'il n'y paraît.

\section{Les choix méthodologiques}

Bien que le projet Relax s'intéresse aux ménages agricoles, nous avons écarté le recours à l'indicateur ménage HDDS et opté pour des scores de diversité alimentaire individuels permettant des interprétations en termes de qualité nutritionnelle du régime alimentaire. Nous avons dans le même temps réfléchi aux conditions permettant de conduire l'analyse au niveau ménage. Le WDDS/MDD-W mesure la diversité alimentaire des femmes en âge de procréer, car celles-ci sont plus vulnérables au risque de malnutrition pour des raisons biologiques (grossesses et allaitement). De plus, la diversité alimentaire de la femme peut être interprétée comme une borne inférieure de ce qui se passe au niveau du ménage, dans la mesure où les inégalités de genre dans l'accès aux ressources alimentaires désavantagent les femmes dans de nombreux contextes. Par ailleurs, afin d'explorer d'éventuelles disparités de consommation alimentaire intra-ménages, nous avons découpé le ménage en plusieurs sous-unités de consommation, et sélectionné aléatoirement un représentant de chaque sous-unité : une femme en âge de procréer (15-49 ans), un homme (15 ans et plus) et un enfant (8-14 ans).

Pour capter les variations saisonnières de la diversité alimentaire des femmes, nous avons procédé à douze mesures mensuelles entre octobre 2017 et septembre 2018; pour les hommes et les enfants, en raison de contraintes budgétaires, nous nous sommes limités à trois mesures, calées sur le calendrier céréalier : février (postrécolte), mai (début de soudure, avec des tensions possibles sur les stocks) et août (premières récoltes). Le nexus «agriculture/nature/marchés/alimentation» étant au cour du projet Relax, des questions relatives au mode d'approvisionnement des aliments ont été intégrées en distinguant quatre modalités : production autoconsommée, achat, collecte et don.

\section{La mise en œuvre sur le terrain}

L'étape préliminaire aux enquêtes a fait l'objet de beaucoup d'attention. D'une manière générale, il est recommandé de prévoir au minimum plusieurs semaines, en ajustant selon le niveau de connaissance du contexte et la documentation disponible.

Dans une première étape, nous avons mené un travail approfondi avec les géographes du projet pour recenser l'ensemble des aliments disponibles au niveau des exploitations, sur les marchés et en brousse. Ces aliments ont été répertoriés dans un glossaire mentionnant le nom scientifique, ainsi que les traductions en français et en plusieurs langues locales, pour faciliter le travail ultérieur des enquêteurs.

Une fois les aliments recensés, ils ont été affectés à l'un des dix groupes alimentaires définis par la méthodologie (note 9). Ce classement n'est pas toujours univoque : 
par exemple, le maïs doit être classé dans le groupe 1 s'il est consommé sous forme de farine (pour la préparation du tô, la pâte qui constitue la base des repas quotidiens) ou dans les «autres légumes» s'il est consommé frais. On doit distinguer également les mangues ou papayes mûres («fruits et légumes riches en vitamines $A »)$ des vertes («autres fruits»). Dans des contextes de très faible diversité alimentaire comme celui du projet, ces différences de classement peuvent avoir un impact important sur le score final.

Enfin, la formation des enquêteurs et enquêtrices est une étape fondamentale, pour maîtriser la dimension technique de l'indicateur, le contexte, mais aussi les aspects relationnels de l'enquête. Un des enjeux est d'obtenir des informations précises sans influencer les réponses. Par exemple, les ingrédients des plats doivent être relevés sans présumer des recettes. La dimension individuelle de l'indicateur ne doit pas être oubliée : lorsque les ingrédients d'un plat peuvent être inégalement distribués lors du partage (viande, légume), on doit s'assurer que la personne interrogée les a bien consommés.

La formation des enquêteurs s'est déroulée sur 6 jours : une première phase pour expliciter la méthode, échanger sur les spécificités locales, et affiner les questionnaires; une phase de jeu de rôles pendant lesquels les participants interprétaient tantôt un enquêteur, tantôt un enquêté; une phase de test en conditions réelles dans un village proche (non inclus dans l'échantillon final).

Lors du premier passage, les personnes enquêtées se sont montrées enthousiastes et curieuses. Les femmes étaient agréablement surprises de l'attention portée à leurs pratiques alimentaires, généralement peu considérées. Au fil des passages, cependant, cet intérêt s'est émoussé et la précision des réponses s'en est ressentie. Nous avons choisi d'enquêter auprès des femmes seules, à leur domicile, pour qu'elles se sentent libres de s'exprimer sur certaines pratiques dévalorisées (par exemple consommer un aliment sans le partager au sein de la famille, ou diluer des sauces faute de moyens). Avec certains interlocuteurs, il a été parfois difficile d'obtenir des réponses fiables en raison de problèmes de compréhension ou de timidité, en particulier lorsque le questionnaire était passé auprès d'enfants non scolarisés ou de femmes installées chez leur belle-famille et ne maîtrisant pas le dialecte local. À plusieurs reprises, les enquêteurs ont dû faire appel à un interprète du village, en veillant à ce qu'il n'influence pas les réponses. Une autre difficulté s'est présentée lorsque la personne interrogée n'avait pas préparé elle-même les plats (cas des enfants, des hommes, et parfois des femmes lorsque le repas avait été préparé par une autre femme du ménage).

La saisie des données s'est faite sur tablette, mais nous avons demandé aux enquêteurs de prendre des notes pour conserver les fiches repas correspondant à chaque enquête, et pouvoir les croiser avec les tableaux d'aliments. Ce dispositif nous a permis de mieux contrôler la qualité des données.

\section{L'analyse des données}

Dans le cadre du projet Relax, nous avons construit le WDDS-10 ainsi que le MDD-W. Si le MDD-W est validé comme indicateur d'adéquation nutritionnelle, son caractère dichotomique le rend moins maniable que le WDDS, notamment 
dans les cas où il présente peu de variations et où les échantillons sont de petite taille. Dans le projet Relax, pour 6 des 12 mois relevés, le pourcentage de femmes atteignant le seuil de 5 groupes était inférieur à $20 \%$, ce qui pose des problèmes de comparaison entre groupes pour l'analyse. Le WDDS-10 est plus sensible aux variations et se prête mieux à des modèles prédictifs.

Pour répondre à nos questions de recherche, nous sommes aussi repartis sur les données intermédiaires afin d'identifier les groupes alimentaires et les aliments consommés. Une analyse de la provenance des aliments a permis de relier les informations nutritionnelles à une analyse plus large en termes de pratiques et de mécanismes au sein du nexus agriculture/nature/marchés/alimentation.

La diversité alimentaire des femmes est très faible, avec un score moyen annuel de 3,6 groupes alimentaires consommés (Lourme-Ruiz et al., 2021). Le plat quotidien est le tô (groupe 1, voir note 4) accompagné d'une sauce cuisinée à base de légumes feuilles (souvent des feuilles de baobab, groupe 7), parfois agrémentée de légumes (oignon, chou, tomate, groupe 9), d'arachides (groupe 3) ou de poisson (groupe 5). Le score s'améliore entre février et juin grâce à la disponibilité ponctuelle des mangues (groupe 8), des produits maraîchers (groupe 9), et des fruits cueillis dans la nature (groupe 10). Comme déjà souligné dans d'autres travaux (Hoddinott et Yohannes, 2002; Savy et al., 2006), la variation du score ne peut pas être interprétée automatiquement comme une variation de la SAN. Il prend sa valeur la plus élevée en juin, alors que de nombreux ménages font face à des pénuries pour les céréales de base : les stocks constitués à la récolte précédente sont insuffisants pour assurer la soudure jusqu'à la récolte suivante, et la capacité d'achat sur les marchés est limitée par la faible trésorerie, d'autant que les prix connaissent une hausse saisonnière en amont de la récolte.

Une entrée par les sources d'approvisionnement fait apparaître des complémentarités entre production, achats et cueillette, avec des schémas complexes et saisonnalisés, qui dépendent des systèmes d'agriculture, d'élevage, d'activités non agricoles, et de la place des femmes dans ces systèmes. Par exemple, la production maraîchère peut impliquer des concurrences pour l'usage des terres et de la main-d'œuvre familiale (notamment vis-à-vis du coton ou des céréales, qui sont la base des systèmes de production locaux); l'étalement de la production maraîchère sur l'année est conditionné par l'accès (rare) à des infrastructures d'irrigation; et la consommation d'aliments périssables (fruits et légumes) est concentrée sur les périodes de récolte ou de cueillette, en raison du manque de moyens de conservation. Ainsi, les mangues, qui représentent la quasi-totalité du groupe des fruits et légumes riches en vitamine A, ne sont disponibles qu'en mars-avril.

Certains groupes interrogent par leur rareté dans l'alimentation des femmes, malgré leur intérêt nutritionnel et leur disponibilité locale. Le lait, produit localement par l'ethnie peule et disponible sur les marchés, n'est pratiquement pas consommé. Les œufs sont totalement absents, alors que les volailles sont présentes dans presque toutes les exploitations. Les légumineuses sont très peu consommées, alors qu'on les trouve en culture pluviale dans nombre d'exploitations, et qu'elles se prêtent bien au stockage. Les contraintes financières et de disponibilité n'expliquent pas tout. Comprendre les mécanismes sous-jacents de cette sous-consommation, pour tenter d'y remédier, suppose de mobiliser des regards pluridisciplinaires sur les 
connaissances, les perceptions et les préférences alimentaires, sur les arbitrages économiques (choisir de vendre plutôt que d'autoconsommer, prioriser les achats de tel ou tel aliment), les éventuelles tensions intra-ménage sur le contrôle et l'usage des budgets et des stocks alimentaires, etc.

Si l'on compare les trois sous-populations de l'échantillon (hommes, femmes, enfants), la consommation à domicile est similaire, mais la consommation hors domicile est discriminante. À la différence des femmes, les enfants consomment plus de légumineuses, grâce au niébé servi dans les cantines scolaires, et plus de fruits cueillis en brousse. Les hommes, qui mènent davantage d'activités hors de la sphère familiale, ont accès à plus de poisson et de légumes tels que le chou, servis dans les petits restaurants informels. Notons que le HDDS, qui exclut la consommation hors foyer, n'aurait pas permis d'identifier ces différences importantes sur le plan nutritionnel.

L'exemple du projet Relax illustre l'intérêt de revenir sur les données en amont des scores, et d'explorer les faisceaux de causes conduisant à la consommation (ou non) d'un groupe ou d'un aliment donné. Les entretiens conduits par les sociologues du projet ont par ailleurs mis en évidence que les ménages ne cherchent pas à «diversifier» leur alimentation au sens des nutritionnistes. La priorité reste d'assurer l'apport en céréales. Si la satiété est assurée, les ménages cherchent à «varier» les plats, en alternant les sauces ou en remplaçant le tô de maïs par du riz. Ces choix ne se traduisent pas nécessairement par une augmentation du score de diversité. D’une manière générale, le score de diversité n'a pas de sens pour les populations qui ne consomment pas des groupes alimentaires, mais des aliments et des plats. Ce travail sur les contraintes, les logiques et les représentations permet de réfléchir à des interventions et à des messages plus appropriés.

Pour conclure ce chapitre, les discussions autour des indicateurs pointent souvent la tension entre standardisation de la méthode (pour produire - dans un temps relativement rapide - des résultats validés et comparables dans le temps et dans l'espace) et adaptation au contexte local (pour gagner en finesse). Dans ce chapitre, nous montrons que le potentiel des indicateurs de SAN tient aussi à la possibilité d'analyser en tant que telles les données collectées en amont pour leur construction, et de les relier à des schémas explicatifs plus parlants. Sans éluder les difficultés qui peuvent surgir, le chapitre souligne l'intérêt d'une collaboration plus étroite entre sciences de la nutrition et sciences sociales, pour produire de la connaissance pertinente et concevoir ou piloter des interventions adaptées en matière de sécurité alimentaire et nutritionnelle.

\section{" Références}

Ballard T., Coates J., Swindale A., Deitchler M., 2011. Indice domestique de la faim. Définition de l'indicateur et guide de mesure, FANTA III - FHI 360, Washington D.C., 26 p.

Coates J., Swindale A., Bilinsky P., 2007. Échelle de l'accès déterminant l'insécurité alimentaire des ménages (HFIAS) pour la mesure de l'accès alimentaire des ménages : guide d'indicateurs, FANTA USAID, Washington D.C., 38 p.

FAO, s.d. The Food Insecurity Experience Scale. [En ligne] http://www.fao.org/3/a-i7835e.pdf (dernière consultation le 27/08/2021). 
FAO, OMS, 1992. Nutrition: the global challenge. Rome, FAO, 33 p.

FAO, 2021. Minimum dietary diversity for women. An updated guide to measurement - from collection to action. Rome, FAO, 176 p. https://doi.org/10.4060/cb3434en

Hoddinott J., Yohannes Y., 2002. Dietary Diversity as a Food Security Indicator, IFPRI, Washington D.C., $81 \mathrm{p}$.

Jones A. D., 2017. Critical review of the emerging research evidence on agricultural biodiversity, diet diversity, and nutritional status in low- and middle-income countries. Nutrition Reviews, 75 (10), 769-782. https://doi.org/10.1093/nutrit/nux040

Kennedy G., Ballard T., Dop M.-C., 2012. Guide pour mesurer la diversité alimentaire au niveau du ménage et de l'individu, FAO, Rome, $56 \mathrm{p}$.

Leroy J., Ruel M., Frongillo E., Harris J., Ballard T., 2015. Measuring the Food Access Dimension of Food Security: a Critical Review and Mapping of Indicators. Food and Nutrition Bulletin, 36 (2), 167-195. https://doi.org/10.1177/0379572115587274

Lourme-Ruiz A., Koffi C. K., Gautier D., Bahya-Batinda D., Bouquet E., Dury S., Martin-Prével Y., Savy M., 2021. Seasonal variability of women's dietary diversity and food provisioning: a cohort study in rural Burkina Faso. Public Health Nutrition, 1-31. https://doi.org/10.1017/S1368980021004171

Maxwell D., Caldwell R., 2008. The Coping Strategies Index. A tool for rapid measurement of household food security and the impact of food aid programs in humanitarian emergencies. Field Method Manual, Tufts University, TANGO International, Care, WFP, 52 p.

Ruel M., Alderman H., 2013. Nutrition-sensitive interventions and programmes: how can they help accelerate progress in improving maternal and child nutrition? Lancet, 2013 (382), 536-551. https://doi.org/10.1016/S0140-6736(13)60843-0

Savy M., Martin-Prével Y., Traissac P., Eymard-Duvernay S., Delpeuch F., 2006. Dietary Diversity Scores and Nutritional Status of Women Change during the Seasonal Food Shortage in Rural Burkina Faso. The Journal of Nutrition, 136 (10), 2625-2632. https://doi.org/10.1093/jn/136.10.2625

Sibhatu K. T., Qaim M., 2018. Review: Meta-analysis of the association between production diversity, diets, and nutrition in smallholder farm households. Food policy, 77, 1-18.

https://doi.org/10.1016/j.foodpol.2018.04.013

Verger E. O., Ballard T. J., Dop M. C., Martin-Prével Y., 2019. Systematic review of use and interpretation of dietary diversity indicators in nutrition-sensitive agriculture literature. Global Food Security, 20, 156-169. https://doi.org/10.1016/j.gfs.2019.02.004

World Food Program, 2008. Food consumption analysis. Calculation and use of the food consumtion score in food analysis, Rome, World Food Program, 24 p.

\section{Liste des principaux sigles et abréviations}

CSI : Index de stratégie de gestion de la vulnérabilité

FANTA : Food and Nutrition Assistance Project

FAO : Organisation des Nations unies pour l'alimentation et l'agriculture

FCS : Score de consommation alimentaire

FIES : Échelle de mesure de l'insécurité alimentaire vécue

HDDS : Score de diversité alimentaire des ménages

HFIAS : Échelle de l'accès déterminant l'insécurité alimentaire des ménages

HHS : Échelle de la faim des ménages, extrait du HFIAS

IDDS : Score de diversité alimentaire individuel

MDD-W : Score minimum de diversité alimentaire des femmes

OMS : Organisation mondiale de la santé

PAM : Programme alimentaire mondial

SAN : Sécurité alimentaire et nutritionnelle

WDDS : Score de diversité alimentaire des femmes 
Les auteures remercient Yves Martin-Prével et Mathilde Savy (IRD) pour leurs suggestions sur une version antérieure du chapitre.

Ce chapitre a été produit dans le cadre du projet Relax : Promoting resilience in the African rural households: food systems at a crossroads. Le projet Relax ( $\mathrm{n}^{\circ}$ AF 1507-329; $\mathrm{n}^{\circ}$ FC 2015-2440, $n^{\circ}$ FDNC Engt 00063479) est financé dans le cadre de l'initiative «Thought for Food » par Agropolis Fondation (sur le programme Investissement d'avenir, financement ANR-10-LABX-0001-01), par Fondazione Cariplo et la Fondation Daniel et Nina Carasso. 



\title{
Chapitre 3 \\ L'évaluation d'impact des actions de prévention et de promotion de la santé nutritionnelle
}

\author{
Marion Tharrey ${ }^{a}$, Mathilde SaVYa ${ }^{a}$, Marlène PERIGNON", \\ CAROLINE MÉJEAN ${ }^{a}$ et NiCOLE DARMON ${ }^{a}$
}

a MoISA, Université Montpellier, CIHEAM-IAMM, CIRAD, INRAE, Institut Agro, IRD, Montpellier, France.

La santé publique a pris un tournant préventif depuis une trentaine d'années. Ce chapitre expose différentes méthodes visant à évaluer les impacts des interventions dans le domaine de la prévention et de la promotion de la santé publique nutritionnelle. Les connaissances produites par ces méthodes permettent in fine d'informer la prise de décision dans ce domaine.

L'histoire de la prophylaxie, la démarche visant à prévenir l'apparition des maladies, est aussi ancienne que celle de la médecine. Jusqu'aux grandes innovations de l'ère industrielle (hygiène publique, vaccination, pasteurisation, antibiotiques, etc.), la démarche préventive en santé a été intimement liée à la lutte contre les maladies infectieuses épidémiques. Elle a pris un tournant nouveau au $\mathrm{Xx}^{\mathrm{e}}$ siècle. En 1948, l'Organisation mondiale de la santé (OMS) définit la santé comme : «un état de complet bien-être physique, mental et social [qui] ne consiste pas seulement en une absence de maladie ou d'infirmité». Cette conception de la santé en tant que bien-être multidimensionnel dépasse la vision réductrice qui consistait jusqu'alors à définir la santé comme l'absence de maladies ou d'infirmité. Les concepts majeurs de prévention et promotion de la santé vont découler de cette définition. La prévention inclut «l'ensemble des mesures visant à éviter ou réduire le nombre et la gravité des maladies, des accidents et des handicaps» (WHO, 1948). On distingue trois types de prévention : la prévention primaire, dont l'objectif est de diminuer l'incidence d'une maladie (i.e. le risque d'apparition de la maladie dans une population); la prévention secondaire, qui vise à diminuer la prévalence d'une maladie (i.e. le nombre de personnes malades); et la prévention tertiaire, qui agit sur les incapacités chroniques liées à la maladie et sur les facteurs de risques de récidive. En 1986 se tient à Ottawa la première conférence internationale pour la promotion de la santé, qui marque un changement de paradigme en santé publique, en allant de la prévention 
de la maladie vers la promotion de la santé. La charte d'Ottawa, qui résulte de cette conférence, constitue le texte fondateur de la promotion de la santé et définit celleci comme un «processus qui confère aux populations les moyens d'assurer un plus grand contrôle sur leur propre santé, et d'améliorer celle-ci» (WHO, 1986). Dans le domaine de la nutrition, la promotion de la santé couvre ainsi une vaste gamme d'interventions sociales, environnementales et économiques visant à renforcer les aptitudes et les capacités des individus afin de limiter les inégalités de santé en lien avec l'alimentation et d'assurer le bien-être des individus. S'insérant dans le champ de l'épidémiologie évaluative, l'objectif de l'évaluation en prévention et promotion de la santé est d'étudier les effets de ces interventions sur la santé, l'alimentation et ses déterminants, afin de s'assurer que ces interventions sont efficaces sur les populations étudiées, qu'elles n'aient pas d'effets adverses et de proposer d'éventuelles pistes d'amélioration. Dans le langage commun, on désigne par le terme d' «évaluation d'impact» l'analyse des effets induits par une intervention ou un programme sur la santé des individus. Ces effets englobent à la fois les effets immédiats (outputs), les effets à moyen terme (outcomes) et les effets à long terme (impacts). Lévaluation d'impact étudie les liens de causalité (i.e. la relation de cause à effet) entre une intervention (existante ou mise en place pour l'étude) et l'évolution de l'état de santé d'un groupe d'individus, afin de déterminer si cette évolution est directement attribuable à l'intervention ou non.

\section{Mesurer l'effet d'une intervention : l'inférence causale}

La difficulté majeure de l'évaluation d'impact est de réussir à isoler l'effet de l'intervention afin de s'assurer que les changements observés ne sont pas attribuables à d'autres facteurs, tels que l'environnement ou les caractéristiques socio-économiques des participants. Pour mesurer l'effet supposé d'une intervention, il faudrait comparer l'évolution d'un paramètre de santé d'un individu si cet individu avait subi l'intervention ou s'il ne l'avait pas subie. Or cette comparaison est impossible car, à un instant donné, un individu ne peut pas être à la fois exposé et non exposé à une intervention de santé. Le lien de cause à effet n'est donc pas directement mesurable et il faut essayer de l'estimer : c'est ce que l'on appelle «l'inférence causale». Un certain nombre de critères peuvent aider à distinguer une relation causale d'une simple association. Les plus connus sont les critères de Hill, établis par Bradford Hill en 1965. Celui-ci distingue 9 critères minimum pouvant aider à l'inférence causale : la force de l'association, la temporalité de l'association (i.e. l'exposition au facteur présumé causal doit précéder l'apparition de la maladie), l'existence d'une relation dose-effet (i.e. le risque doit augmenter avec le niveau d'exposition au facteur), la spécificité de l'association (i.e. une cause conduit à un effet particulier), la reproductibilité de l'association dans différentes populations ou contextes, la plausibilité biologique de l'association (i.e. l'association considérée est cohérente par rapport aux connaissances biologiques générales), la cohérence biologique (i.e. l'interprétation causale de l'association considérée ne se retrouve pas en contradiction avec les connaissances disponibles), la présence de données expérimentales biologiques ou animales, et l'analogie par rapport à d'autres relations causales et à leurs mécanismes. 
Concernant la mesure, comme dans toutes les études, il y a un risque que la valeur mesurée d'un effet (ici l'effet causal) soit sujette à une erreur systématique, appelée aussi biais. Ces biais sont multiples et peuvent intervenir à différents niveaux, que ce soit lors de la sélection des sujets de l'étude (biais de sélection), du recueil des informations (biais de mesure) ou de l'analyse des données (biais de confusion) (tableau 3.1). L'évaluation d'impact nécessite donc de mettre en place des méthodes d'évaluation qui vont permettre d'estimer l'effet causal d'une intervention sur la santé d'une population donnée tout en limitant les biais mentionnés précédemment. Il existe deux grandes familles de méthodes d'évaluation : les études expérimentales et les études quasi expérimentales.

Les études expérimentales, appelées aussi essais contrôlés randomisés (de l'anglais randomized controlled trial ou $R C T$ ), sont largement utilisées en médecine expérimentale afin de mesurer l'efficacité d'un traitement. Ces méthodes consistent à comparer l'évolution d'une (ou plusieurs) variable(s) d'intérêt dans un groupe qui reçoit l'intervention à l'évolution de cette/ces même(s) variable(s) d'intérêt dans un groupe qui ne reçoit pas l'intervention (appelé groupe contrôle, groupe témoin ou encore groupe de référence). Avec la «randomisation», la répartition des individus dans le groupe d'intervention ou dans le groupe contrôle se fait de manière aléatoire. Le chercheur contrôle donc les différents paramètres de l'étude, ainsi que l'accès du groupe à l'intervention. La variable d'intérêt peut être évaluée à plusieurs intervalles de temps, a minima avant et après l'intervention, et éventuellement pendant l'intervention. La randomisation permet de former des groupes comparables. En effet, pour un échantillon suffisamment grand, l'affectation aléatoire assure des distributions qui tendent à être similaires pour tous les facteurs qui ne sont pas affectés par le traitement. La randomisation tend ainsi à égaliser les caractéristiques des individus entre les groupes. Les conditions de l'expérimentation étant contrôlées et les groupes comparables, les risques de biais de sélection et de confusion sont réduits et l'effet de l'intervention sur l'état de santé des individus peut être plus facilement isolé. On dit que les essais contrôlés randomisés ont une forte validité interne ${ }^{11}$. Ce type d'étude est considéré comme étant à fort niveau de preuve (Haute Autorité de santé, 2013), et se trouve ainsi désigné comme la méthode de référence (ou gold standard) en évaluation d'impact. Idéalement, les essais contrôlés randomisés sont réalisés de manière à ce que soit l'investigué seulement (essai en simple aveugle), soit l'investigué et l'investigateur (essai en double aveugle) ne sachent pas dans quel groupe se trouve la personne investiguée. Cette procédure permet d'éviter des biais de mesure liés à l'investigué (qui risque de réagir différemment en fonction du groupe auquel il appartient), mais aussi à l'investigateur (qui peut porter une attention supplémentaire au groupe d'intervention).

Les études quasi expérimentales s'apparentent à des études expérimentales, mais en diffèrent par l'absence de randomisation des individus dans le groupe d'intervention

11. Une étude a une bonne validité interne si le résultat obtenu est fiable, c'est-à-dire que les résultats mesurés sont bien ceux attribués à l'intervention. Il existe deux types d'erreurs principales, qui pourraient menacer la validité interne d'un essai : il s'agit du biais (également appelé erreur systématique) et de l'erreur aléatoire (également appelée erreur fortuite ou erreur statistique). La qualité méthodologique de l'étude dépend de la présence ou de l'absence de biais. 
ou le groupe contrôle. Le chercheur doit alors tenter de reproduire les conditions d'une expérimentation à l'aide d'une conception d'étude appropriée (appelée traditionnellement «design») et d'une analyse statistique pertinente.

Tableau 3.1. Les biais d'enquêtes.

\begin{tabular}{|c|c|c|}
\hline Type de biais & Définition & Exemples de biais \\
\hline Biais de sélection & $\begin{array}{l}\text { Intervient lors de la sélection } \\
\text { des participants : } \\
\text { Échantillon non représentatif } \\
\text { de la population cible } \\
\text { Différences systématiques } \\
\text { entre les divers groupes de } \\
\text { comparaison d'une étude }\end{array}$ & $\begin{array}{l}\text { Biais de volontariat : les individus } \\
\text { volontaires ont des caractéristiques } \\
\text { différentes de ceux refusant de participer } \\
\text { à l'étude } \\
\text { Biais d'attrition : les individus } \\
\text { abandonnant l'étude avant la fin } \\
\text { ont des caractéristiques différentes } \\
\text { de ceux restants }\end{array}$ \\
\hline $\begin{array}{l}\text { Biais de mesure } \\
\text { (ou d'information) }\end{array}$ & $\begin{array}{l}\text { Erreur d'estimation } \\
\text { au moment de la collecte } \\
\text { des données, qui intervient } \\
\text { de manière identique } \\
\text { (erreur non différentielle) } \\
\text { ou différente (erreur } \\
\text { différentielle) dans les groupes } \\
\text { de comparaison d'une étude }\end{array}$ & $\begin{array}{l}\text { Biais de déclaration : déclaration } \\
\text { erronée ou imprécise des individus, due } \\
\text { à un problème de mémoire ou d'omission } \\
\text { volontaire (biais de sous-déclaration) } \\
\text { Biais liés à l'imprécision de la méthode : } \\
\text { par exemple, un questionnaire } \\
\text { de fréquence alimentaire non validé } \\
\text { conduisant à une estimation erronée } \\
\text { des portions consommées } \\
\text { Biais de désirabilité sociale : tendance } \\
\text { des individus à donner des réponses } \\
\text { socialement désirables } \\
\text { Biais de subjectivité de l'enquêteur : } \\
\text { attention supplémentaire de l'enquêteur } \\
\text { pour le groupe d'intervention }\end{array}$ \\
\hline Biais de confusion & $\begin{array}{l}\text { Estimation erronée } \\
\text { des associations due à } \\
\text { un facteur extérieur associé } \\
\text { à la fois à l'exposition et } \\
\text { à l'état de santé }\end{array}$ & \\
\hline
\end{tabular}

Les études expérimentales et quasi expérimentales peuvent être explicatives ou pragmatiques. La recherche explicative (efficacy trial) vise à évaluer si une intervention fonctionne dans des conditions idéales ou sélectionnées, tandis que la recherche pragmatique vise à évaluer si l'intervention fonctionne dans des conditions réelles (effectiveness trial). Ces deux notions d'efficacité (efficacy) ou d'efficience (effectiveness) sont importantes à considérer lors de la conception d'une étude en prévention ou promotion de santé car elles détermineront les questions méthodologiques clés relatives au protocole de l'intervention (type de design, lieu d'intervention, population ciblée, choix du groupe contrôle, etc.). Les expérimentations naturelles ${ }^{12}$ sont un exemple de recherches dites pragmatiques.

12. Une expérimentation naturelle est une expérience dans laquelle l'exposition à l'intervention est provoquée par des causes naturelles et/ou politiques qui ne sont pas manipulables par le chercheur. 


\section{" Évaluer l'effet d'une intervention: une diversité de méthodes}

\section{Conception de l'étude (ou design)}

En pratique, différents designs expérimentaux ou quasi expérimentaux peuvent être envisagés lors de la réalisation d'une étude d'évaluation d'impact.

\section{Designs expérimentaux}

Divers designs expérimentaux peuvent être envisagés en fonction du contexte, des variables mesurées et de la population d'étude. Les designs expérimentaux les plus courants sont : les essais contrôlés en groupes parallèles, les essais contrôlés randomisés en clusters, les essais contrôlés randomisés avec permutation séquentielle, ou encore les essais randomisés avec consentement post-randomisation. Nous allons détailler par la suite ces différents designs.

\section{Les essais contrôlés en groupes (ou bras) parallèles}

Le design standard des essais contrôlés randomisés est l'essai en groupes (ou bras) parallèles (parallel-group randomized trial) inspiré des essais thérapeutiques qui visent à tester l'efficacité d'un nouveau traitement (figure 3.1). Ce design consiste à assigner aléatoirement les participants dans un groupe qui reçoit l'intervention ou dans un groupe contrôle et à les suivre en parallèle.

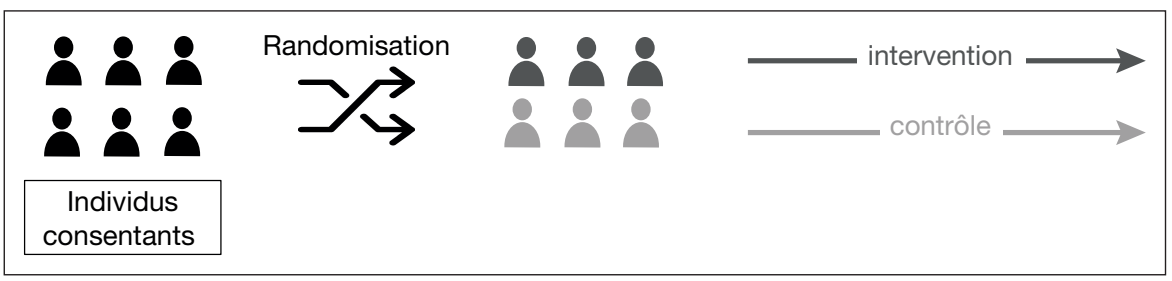

Figure 3.1. Design d'un essai contrôlé randomisé en groupes parallèles.

L'étude SU.VI.MAX, présentée ci-dessous (encadré 3.1), est un exemple d'essai contrôlé randomisé en groupes parallèles.

Les interventions en prévention ou promotion de la santé ont souvent une visée plus large qu'un effet à l'échelle individuelle et sont constituées de composantes multiples susceptibles d'interagir entre elles d'abord, et ensuite avec les contextes dans lesquels elles sont mises en œuvre. Leur complexité entraîne souvent des contraintes méthodologiques, d'ordre pratique ou éthique, empêchant la réalisation des essais en bras parallèles. Nous allons voir, par la suite, d'autres designs expérimentaux couramment utilisés en santé publique pour évaluer l'efficacité d'une intervention.

\section{Essais contrôlés randomisés en clusters (ou grappes)}

Lorsque l'intervention vise des groupes d'individus ou des entités (comme des écoles, des hôpitaux, des villes, etc.), la randomisation à l'échelle de l'individu n'est 


\section{Encadré 3.1 : L'étude SU.VI.MAX (Hercberg et al. 2004)}

\section{Objectif}

Évaluer l'effet d'une supplémentation combinant vitamines et minéraux antioxydants sur la réduction de l'incidence du cancer et des maladies cardiovasculaires ischémiques en population générale.

\section{Plan expérimental}

Au total, 13017 adultes français de 35 à 60 ans ont été recrutés sur la base du volontariat. Tous les participants ont pris une seule capsule par jour combinant des vitamines et minéraux antioxydants ou un placebo. Lattribution aléatoire du traitement a été réalisée par génération de séquences en bloc stratifiées par sexe et groupe d'âge. Lessai a été réalisé en double aveugle. Des prélèvements sanguins, des mesures anthropométriques et la déclaration de nouveaux événements de santé (cancers, maladies cardiovasculaires) ont été réalisés à différents intervalles de temps entre le début et la fin de l'intervention. La durée médiane du suivi était de 7,5 ans.

pas la plus adaptée et il est plus pertinent de randomiser directement les entités plutôt que les individus. On parle alors d'essais randomisés en clusters ou grappes (cluster randomized trial) (figure 3.2).

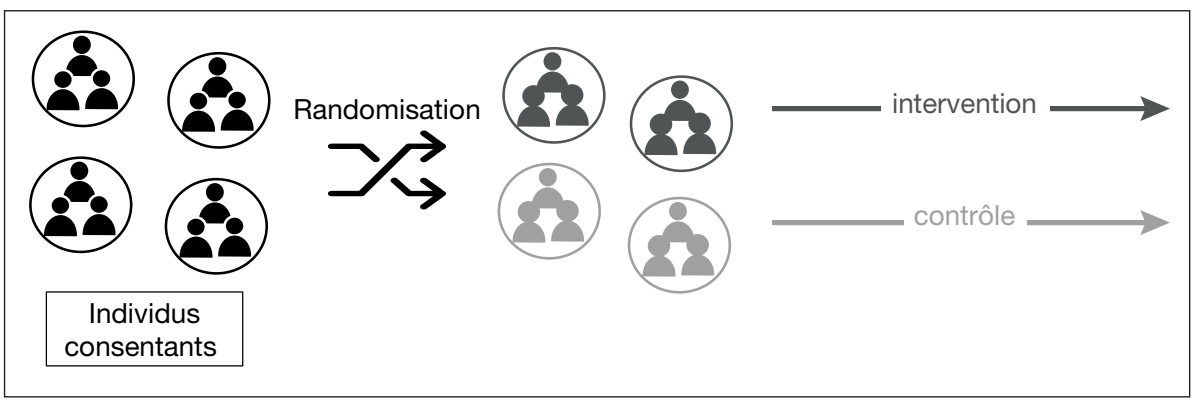

Figure 3.2. Design d'un essai contrôlé randomisé en clusters.

La randomisation en clusters offre l'avantage de pouvoir séparer géographiquement le groupe d'intervention du groupe contrôle. Ce design est donc à privilégier lorsque le risque de contamination entre les groupes (i.e. certains individus du groupe contrôle reçoivent l'intervention) est élevé, pouvant entraîner une sous-estimation de l'effet réel de l'intervention. Les individus d'un même cluster ayant tendance à avoir des caractéristiques plus similaires qu'avec les individus des autres clusters, les essais en clusters nécessitent toutefois un nombre plus important d'individus pour tenir compte de cette corrélation intra-clusters. L'étude Cash for Nutrition Awareness, présentée ci-dessous (encadré 3.2), est un exemple d'essai contrôlé randomisé en clusters.

\section{Essais contrôlés randomisés avec permutation séquentielle}

Une limite importante des essais en groupes parallèles ou en clusters est que le groupe contrôle ne bénéficie pas de l'intervention, ce qui soulève un problème éthique. 


\section{Encadré 3.2 : L'étude Cash for Nutrition Awareness au sein du projet SNACK} (Adubra et al. 2019)

\section{Objectif}

Évaluer l'effet de transferts monétaires et/ou de suppléments nutritionnels ciblant la période des «1000 jours» (de la conception aux deux ans de l'enfant), conditionnés à la fréquentation des centres de santé sur la réduction du retard de croissance chez les jeunes enfants, dans la région rurale de Kayes au Mali.

\section{Plan expérimental}

Soixante-seize centres de santé communautaires ont été répartis aléatoirement dans un groupe de comparaison dans lequel les mères ne bénéficiaient que d'activités de base en santé et nutrition (= activités SNACK) et trois groupes intervention dans lesquels les mères recevaient, en plus des activités SNACK :

- des transferts monétaires mensuels;

- des suppléments nutritionnels à base lipidique pour leur enfant de 6 à 23 mois ;

- ou la combinaison des transferts monétaires et des suppléments nutritionnels.

Dans chaque bras d'étude, un échantillon représentatif de couples mères-enfants a été sélectionné et enquêté avant le début de l'intervention (2013, n = 5046) puis 3 ans plus tard, en fin d'intervention $(2016, \mathrm{n}=5098)$.

Une solution peut être d'inclure progressivement les groupes dans l'intervention. On parle alors d'essais randomisés avec permutation séquentielle (stepped wedge randomized trial). Au départ, aucun individu (ou cluster) ne reçoit l'intervention puis progressivement chacun bénéficie de l'intervention dans un ordre aléatoirement prédéterminé. L'effet de l'intervention peut alors être estimé à partir de comparaisons entre les groupes (les individus qui n'ont pas encore subi l'intervention servent de groupe contrôle) ou à l'intérieur des groupes (avant et après intervention), permettant une comparaison intra et inter-groupes de l'effet de l'intervention (figure 3.3).

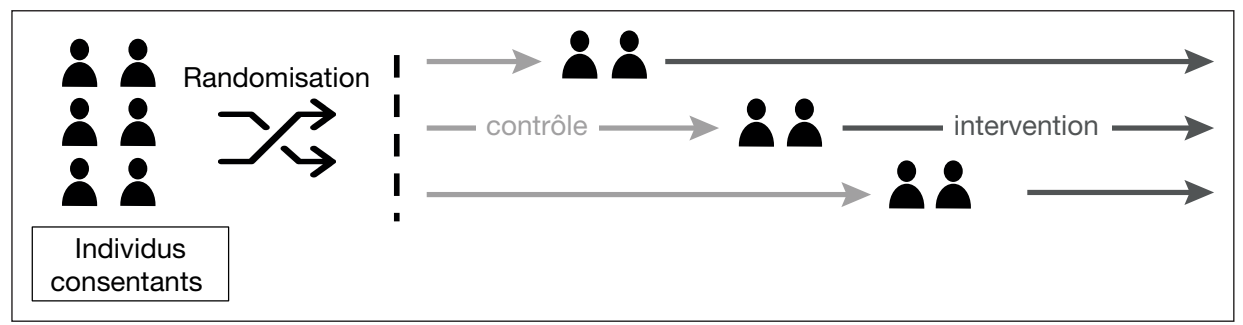

Figure 3.3. Design d'un essai contrôlé randomisé avec permutation séquentielle.

En étalant l'intervention dans le temps, les essais avec permutation séquentielle offrent également la possibilité d'étudier l'effet temporel de l'intervention. Ce type de design peut se révéler particulièrement utile dans le cadre d'interventions qui sont difficiles à mener simultanément sur la moitié de la population, que ce soit pour des raisons éthiques, pratiques, logistiques ou budgétaires. Cependant, sur le long terme, les coûts peuvent être élevés car tous les individus reçoivent l'intervention. De plus, la principale limite des essais avec permutation séquentielle est leur durée 
plus longue que les autres essais du fait de l'inclusion successive des groupes, ce qui peut accroître le taux d'abandon des participants, mais aussi augmenter le risque que des facteurs extérieurs à l'intervention aient évolué dans le temps, rendant la comparaison entre les groupes plus difficile. On parle alors de biais de conjoncture. L'étude présentée ci-dessous (encadré 3.3) est un exemple d'essai contrôlé randomisé avec permutation séquentielle.

\section{Encadré 3.3 : Intervention en milieu scolaire (Ni Mhurchu et al. 2013)}

\section{Objectif}

Évaluer les effets d'un programme de distribution de petits-déjeuners scolaires gratuits sur la fréquentation scolaire, les résultats scolaires, le comportement psychosocial, les habitudes alimentaires, la sensation de faim, les habitudes de petit-déjeuner et la sécurité alimentaire.

\section{Plan expérimental}

Quatorze écoles primaires situées dans quartiers défavorisés de trois régions de Nouvelle-Zélande (Auckland, Waikato et Wellington) et n'ayant pas de programme de petits-déjeuners ont été identifiées en 2010. Au total, 424 élèves de 5 à 13 ans ont rempli les critères d'éligibilité et ont accepté de participer à l'étude. Toutes les écoles ont servi de témoins au début de l'intervention, puis ont été assignées de manière aléatoire à débuter l'intervention lors d'une des quatre périodes scolaires (3-4 écoles par période). À l'issue de l'année scolaire, toutes les écoles avaient bénéficié du programme de distribution de petits-déjeuners.

\section{Essais randomisés avec consentement post-randomisation (schéma de Zelen)}

Outre les problèmes éthiques, les designs en groupes parallèles ou en clusters risquent d'entraîner un abandon accru des participants alloués au groupe contrôle. Proposé pour la première fois par Marvin Zelen, statisticien à l'école de santé publique de Harvard, le schéma de Zelen est un essai randomisé avec consentement post-randomisation. Contrairement aux autres essais, où la randomisation dans le groupe d'intervention ou le groupe contrôle n'est réalisée qu'après consentement éclairé de l'individu, le schéma de Zelen implique de randomiser les individus avant de leur avoir demandé leur consentement. Les individus alloués au groupe d'intervention sont ensuite informés et peuvent refuser de recevoir l'intervention (figure 3.4).

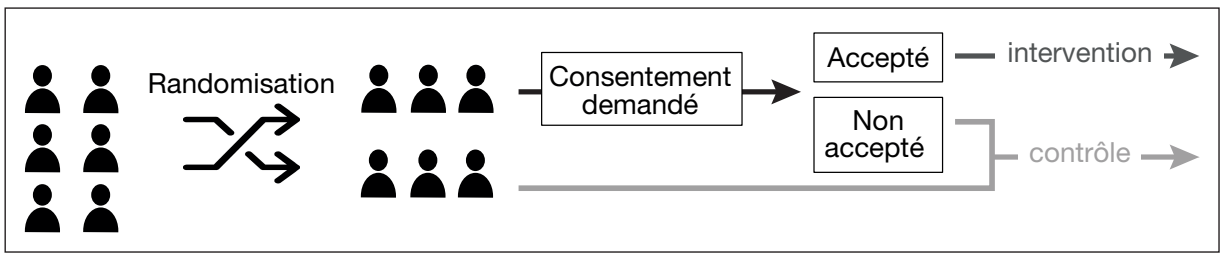

Figure 3.4. Design d'un essai contrôlé randomisé avec consentement post-randomisation. 
L'intérêt majeur du schéma de Zelen est double : il permet de recruter davantage d'individus, car le consentement n'est demandé qu'aux individus du groupe d'intervention, mais aussi de limiter le taux d'abandon en évitant aux participants la déception d'être alloué dans le groupe contrôle (car ils ne savent pas que l'intervention existe). Toutefois, ce design est très peu utilisé car il soulève de nombreuses questions éthiques, notamment le fait que les individus du groupe contrôle soient inclus dans l'étude sans que leur consentement éclairé ait été préalablement recueilli. De plus, ce design implique de collecter les données des participants alloués au groupe d'intervention qui refusent de recevoir l'intervention. Afin de respecter le principe de randomisation, les schémas de Zelen sont analysés en intention de traiter, c'est-à-dire que les résultats sont examinés en fonction des groupes dans lesquels les individus ont été randomisés initialement, quel que soit le traitement réellement reçu. Le principe de l'analyse en intention de traiter est de conserver les bénéfices de la randomisation au moment de l'analyse des résultats afin d'éviter les biais liés aux écarts entre le protocole de l'essai et la conduite de celui-ci, qui ne seraient pas dus au hasard mais seraient en rapport avec le traitement administré. Lors de l'analyse des résultats, les individus assignés au groupe d'intervention sont ainsi comparés à ceux du groupe contrôle sans tenir compte du fait que l'individu ait accepté ou non l'intervention. L'efficacité de l'intervention peut alors être masquée si le taux de refus de participation est élevé. L'étude The Lyon Diet Heart Study, présentée ci-dessous (encadré 3.4), est un exemple d'essai contrôlé randomisé avec consentement post-randomisation.

Encadré 3.4 : The Lyon Diet Heart Study (de Lorgeril et al., 1994)

\section{Objectif}

Comparer l'effet d'un régime alimentaire méditerranéen riche en acide alphalinolénique à celui d'un régime normal sur le taux de récidive après un premier infarctus du myocarde.

\section{Plan expérimental}

Au total, 605 patients âgés de moins de 70 ans et ayant survécu à un infarctus du myocarde dans les 6 mois précédents ont été recrutés au CHU de Lyon. Pendant leur séjour à l'hôpital, les patients ont accepté de participer à une cohorte de 5 ans sans toutefois être pleinement informés de la conception de l'étude, en particulier de la comparaison entre deux régimes alimentaires. Seuls les patients assignés au groupe expérimental ont signé un second consentement éclairé, dans lequel ils acceptaient de modifier leur régime alimentaire et ont été encouragés à adopter un régime de type méditerranéen riche en acide alpha-linolénique. Pendant les 4 premières années, les habitudes alimentaires ont été évaluées uniquement dans le groupe expérimental, à l'aide de rappels de 24 heures et de questionnaires de fréquence, afin de ne pas influencer le comportement des témoins. Des entretiens médicaux, des prélèvements sanguins et des mesures de la tension artérielle ont été menés à 8 semaines, puis annuellement afin d'identifier les décès par cause cardiovasculaire, les infarctus non mortels, et l'apparition de troubles vasculaires, tels que l'angine de poitrine, l'insuffisance cardiaque, les accidents vasculaires cérébraux ou encore les embolies pulmonaires. 


\section{Designs quasi expérimentaux}

Les principaux designs non expérimentaux utilisés dans les interventions en prévention et promotion de la santé nutritionnelle sont les essais contrôlés non randomisés et les séries chronologiques. Nous allons détailler par la suite ces différents designs.

\section{Les essais contrôlés non randomisés}

Bien que les essais contrôlés randomisés soient considérés comme le plan d'étude optimal pour minimiser les biais et fournir une estimation précise de l'impact d'une intervention, de nombreuses contraintes empêchent leur utilisation, en santé publique notamment. Le chercheur n'est pas toujours en mesure de contrôler le lieu et l'exposition à l'intervention, qui peuvent avoir été sélectionnés avant le début de l'étude d'évaluation. Les commanditaires de l'étude peuvent également s'opposer à une répartition aléatoire des individus et vouloir servir toutes les personnes nécessiteuses de l'intervention, rendant difficile la constitution d'un groupe contrôle. Ainsi, lorsqu'il n'est pas possible de répartir aléatoirement les individus entre le groupe d'intervention et le groupe contrôle, les essais contrôlés non randomisés (également appelés études avant/après avec groupe contrôle) peuvent alors être envisagés pour étudier des inférences causales (figure 3.5).

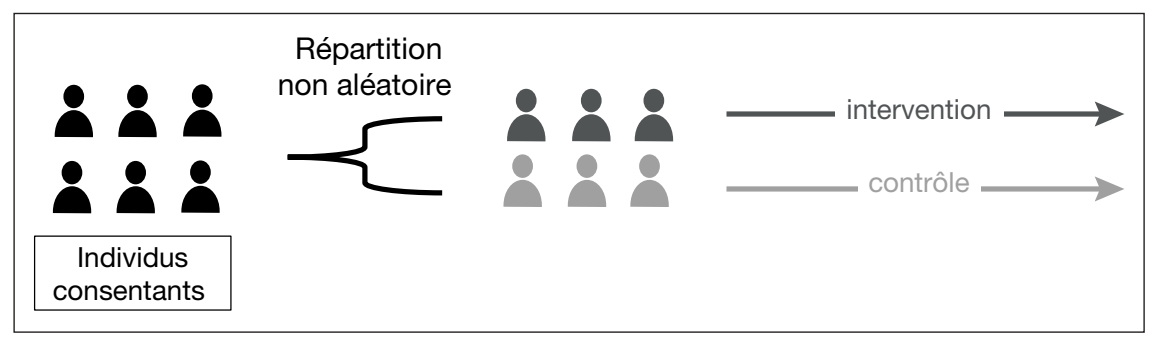

Figure 3.5. Design d'un essai contrôlé non randomisé.

La principale limite de ce type d'étude est le risque de biais lié à l'absence de randomisation, pouvant conduire à des groupes non comparables avant l'intervention. En particulier, les individus participant à l'intervention peuvent différer des individus du groupe contrôle sur certaines caractéristiques, comme des critères sociodémographiques. Les différences d'état alors observées entre les groupes ne seront peut-être pas dues à l'intervention mais au fait que les groupes n'étaient pas comparables au préalable. Ces biais portent atteinte à la validité interne de l'étude rendant l'évaluation de la causalité plus difficile. Leur niveau de preuve est donc inférieur aux essais randomisés contrôlés (Haute Autorité de santé, 2013). Leétude JArDinS, présentée ci-dessous (encadré 3.5), est un exemple d'essai contrôlé non randomisé.

\section{Les séries chronologiques}

Suivant le contexte, il peut être difficile, voire impossible, d'identifier un groupe contrôle qui ne soit pas exposé à l'intervention (par exemple, lors de l'adoption d'une nouvelle loi ou de la mise en place d'une nouvelle campagne de prévention). En l'absence de groupe contrôle, une simple mesure du paramètre de santé étudié avant et après la mise en place de l'intervention ne permettra pas de mettre en évidence 
Encadré 3.5 : L'étude JArDinS (Tharrey et al., 2020)

\section{Objectif}

Évaluer l'impact de la première année de participation à un jardin partagé sur les 3 dimensions de la durabilité (santé/social, environnement, économie) des styles de vie.

\section{Plan expérimental}

Soixante-quinze individus débutant dans un jardin partagé à Montpellier ont été recrutés en 2018. Un groupe contrôle de non-jardiniers a été créé en invitant des volontaires participant à une enquête sur les comportements d'approvisionnement alimentaire à Montpellier à contribuer à l'étude. Chaque jardinier a été apparié à un non-jardinier selon les critères d'appariement suivants : tranche d'âge, sexe, structure du foyer, revenus du foyer et typologie du quartier de résidence. Des données à l'échelle du foyer (qualité nutritionnelle, impact environnemental et coût des approvisionnements alimentaires sur 1 mois), et des données à l'échelle individuelle (activité physique, bien-être mental, isolement social, sensibilité au gaspillage alimentaire et connexion à la nature) ont été collectées au moment de l'inclusion dans l'étude, puis 1 an après.

une relation de causalité. Afin d'obtenir une estimation non biaisée de l'effet causal de l'intervention, il faut que seule l'intervention fasse varier le(s) paramètre(s) de santé étudié(s). Or, cette hypothèse forte est rarement vérifiée, car l'environnement peut avoir évolué entre le début et la fin de l'intervention, entraînant des modifications de la (ou les) variable(s) d'intérêt (biais de conjoncture). Une solution consiste à collecter des données à plusieurs moments, avant et après l'intervention, afin de détecter si l'intervention a modifié une tendance préexistante : c'est ce qu'on appelle la série chronologique (figure 3.6).
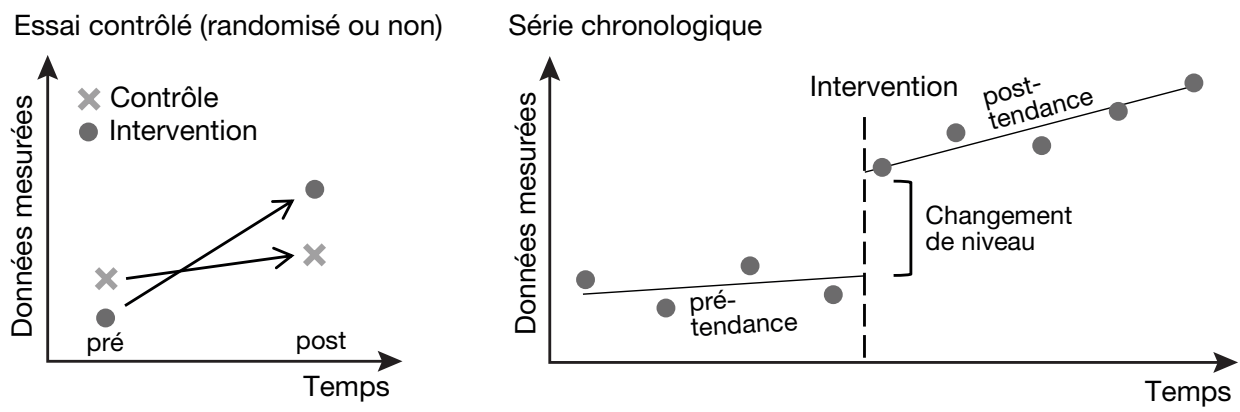

Figure 3.6. Design d'une série chronologique.

Ces études ont un niveau de preuve inférieur par rapport aux essais contrôlés randomisés ou non randomisés (Haute Autorité de santé, 2013). L'avantage des séries chronologiques est qu'elles sont relativement rapides à réaliser si des données de routine sont disponibles et peu coûteuses. Elles sont également à privilégier si on veut observer les effets d'une intervention sur le long terme. Toutefois, même lorsqu'elles sont bien menées, les séries chronologiques ne permettent pas de tenir compte des événements autres que l'intervention qui pourraient expliquer un changement dans 
le paramètre de santé étudié. De plus, elles nécessitent une mobilisation plus importante de l'équipe de recherche, qui doit collecter des données de manière régulière, sur de longues périodes et en utilisant les mêmes méthodes de collecte. Létude présentée ci-dessous (encadré 3.6) est un exemple de série chronologique.

\title{
Encadré 3.6 : Augmentation du prix des boissons sucrées dans une chaîne de restaurants (Cornelsen et al., 2017)
}

\author{
Objectif \\ Évaluer l'impact d'une augmentation de 0,10£ par boisson sur les ventes de \\ boissons sucrées non alcoolisées dans une chaîne nationale de restaurants en \\ Angleterre.
}

\section{Plan expérimental}

Le $1^{\mathrm{er}}$ septembre 2015, une chaîne nationale de restaurants britanniques a augmenté le prix des boissons sucrées de $0,10 £$ (par boisson vendue). Pour évaluer l'efficacité de cette expérimentation naturelle, des données chronologiques détaillées sur le nombre de boissons vendues avant et après la mise en place de l'intervention dans 37 restaurants éligibles ont été fournies par la société. Les variations du nombre de boissons sucrées non alcoolisées et d'autres boissons vendues par client à court terme et à long terme ont été mesurées en utilisant respectivement des données hebdomadaires (12 semaines avant et après la mise en place de l'intervention) et mensuelles (6 mois avant et après la mise en place de l'intervention).

Le tableau 3.2 résume les différents designs présentés dans cette section, ainsi que leurs avantages et inconvénients respectifs.

\section{Augmenter l'homogénéité entre les groupes: la solution de l'appariement}

Comme nous l'avons évoqué dans la section précédente, plus le nombre d'individus est élevé, plus la randomisation constitue des groupes similaires du fait de la loi des grands nombres ${ }^{13}$. Cependant, des contraintes techniques et budgétaires peuvent limiter le recrutement des participants. Afin d'améliorer l'homogénéité entre le groupe d'intervention et le groupe contrôle sans augmenter la taille de l'échantillon, une randomisation stratifiée peut être une solution. Dans ce cas, les participants sont regroupés en strates selon un ou plusieurs facteurs et la randomisation est effectuée au sein de chaque strate. On appelle «appariement» ce regroupement de participants en fonction de facteurs jugés pertinents. Prenons l'exemple d'une étude s'intéressant à l'effet de la consommation de fruits et légumes sur le risque de développer un cancer du sein. Les traitements hormonaux de la ménopause pouvant augmenter le risque de cancers du sein, il peut être important de stratifier en fonction du statut ménopausique afin que chaque groupe compte la même proportion de femmes en préménopause et en postménopause.

13. La loi des grands nombres est un théorème mathématique qui exprime le fait que les caractéristiques d'un échantillon aléatoire se rapprochent des caractéristiques statistiques de la population lorsque la taille de l'échantillon augmente à l'infini. 
Tableau 3.2. Avantages et inconvénients des différents designs d'études expérimentales et quasi expérimentales.

\section{Essais contrôlés randomisés}

NTAGES

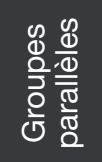

- Protocole relativement simple et méthodologie robuste

- Durée de participation courte pour chaque participant

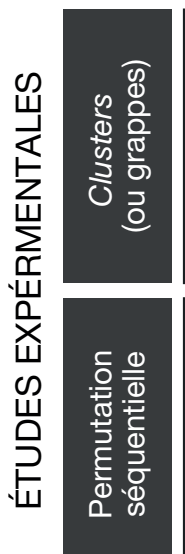

- Adapté à l'évaluation d'interventions qui ciblent des groupes d'individus

- Limite le risque de contamination

-Tous les groupes bénéficient de l'intervention

- Permet d'étudier l'effet temporel de l'intervention

- Permet d'étaler les contraintes logistiques et/ou budgétaires dans le temps

- Recrutement facilité car pas de consentement pour le groupe contrôle

- Évite aux participants la déception d'être alloué dans le groupe contrôle
INCONVÉNIENTS

- Problème éthique

- Risque d'abandon des participants ne recevant pas l'intervention

- Problème éthique

- Risque de non-comparabilité entre les groupes

- Nécessite un nombre élevé de participants pour tenir compte de l'effet cluster

-Analyses statistiques complexes

- Période d'étude relativement longue : risque d'abandon des participants élevé

- Nécessite de tenir compte de l'effet temps

-Analyse statistiques complexe

- L'effet de l'intervention peut ne pas être observé si le taux de refus de participation est élevé.

- Problème éthique important

\section{Essais contrôlés non randomisés}

\section{AVANTAGES}

- Évaluation possible d'une intervention lorsque la randomisation n'est pas possible.

- Études moins coûteuses que les études expérimentales

- Bonne validité externe dans le cas des expérimentations naturelles

\section{INCONVÉNIENTS}

- Moins bonne validité interne que les études expérimentales

- Risque de biais de sélection : groupe contrôle non semblable

- Risque de biais de confusion

\section{Séries chronologiques}

\section{AVANTAGES}

- Possibilitéde mesurer l'effet d'une intervention qui touche l'ensemble de la population (pas de groupe contrôle)

- Prise en compte des phénomènes périodiques

- Possibilité de suivi permanent 
En général, les facteurs de stratification sont des variables sociodémographiques telles que l'âge, le sexe ou le niveau d'éducation, mais peuvent aussi être des critères relatifs à l'environnement ou aux modes de vie des individus. Le principal avantage de l'appariement est d'équilibrer la distribution du (ou des) facteur(s) entre les groupes permettant de renforcer la comparabilité des groupes vis-à-vis du (ou des) facteur(s) de stratification. L'appariement peut également être utilisé afin de surreprésenter certaines catégories de population qui ont un intérêt particulier pour l'étude.

Dans le cas des études quasi expérimentales, une autre solution pour limiter les biais de confusion dus à l'absence de randomisation consiste à apparier individuellement les individus du groupe contrôle à ceux du groupe d'intervention sur un ou plusieurs facteur(s) de confusion connu(s). Il faut toutefois éviter d'apparier sur un nombre trop important de variables, car cela rend plus difficile, voire impossible, l'identification d'individus similaires pour former le groupe contrôle. De plus, l'appariement sur des variables qui ne sont pas des facteurs de confusion potentiels et qui sont fortement associées à l'exposition tend à rendre comparable les groupes intervention et contrôle vis-à-vis de l'exposition étudiée, conduisant à une perte de puissance et à une sous-estimation de l'effet de l'intervention, on parle alors de sur-appariement. Pour éviter ce problème, il est habituel d'apparier uniquement sur des facteurs potentiels de confusion bien établis dans la littérature. Dans le cas où de nombreuses variables risquent d'influencer à la fois la participation à l'intervention et la variable d'intérêt, une méthode consiste à apparier les individus non pas sur un nombre restreint de caractéristiques observables, mais sur un score, appelé «score de propension». Celuici est calculé à partir d'un ensemble de caractéristiques observées de l'individu et résume l'influence de ces caractéristiques sur la propension à participer à l'intervention. Quelle que soit la méthode choisie, la principale limite de l'appariement est que les variables sélectionnées sont en général des caractéristiques observables des individus. Or, d'autres variables non observables (comme la motivation, les croyances, etc.) peuvent aussi influencer la participation ou non à une intervention et conduire à des estimations biaisées de l'effet causal.

\section{Taille d'échantillon}

Une fois identifiée la méthode d'évaluation la plus adaptée au contexte, un calcul de puissance statistique doit être réalisé afin de déterminer le nombre minimum d'individus à recruter dans l'étude afin d'estimer l'impact de l'intervention. Cette étape est indispensable, car si le nombre d'individus n'est pas suffisant, les effets de l'intervention ne pourront pas être estimés. Plus la taille de l'échantillon est élevée, plus la puissance statistique sera importante. Le calcul de taille d'échantillon dépend de la manière dont l'étude a été conçue, du type de données collectées, du critère principal de jugement choisi pour ce calcul et du niveau de significativité attendu. Il est également important de prévoir le taux d'attrition (c'est-à-dire le taux d'abandon des participants avant la fin de l'étude) et de majorer la taille de l'échantillon en conséquence. En effet, si le taux d'attrition est plus élevé que prévu cela entraînera une perte de puissance statistique pouvant avoir des conséquences méthodologiques importantes. De plus, un taux d'attrition élevé augmente le risque de biais, car les individus restants dans l'étude auront sûrement des caractéristiques différentes de ceux ayant abandonné l'étude. 


\section{" Enjeux déontologiques et juridiques}

Les grands principes éthiques applicables aux recherches menées sur des êtres humains ont été énoncés par la déclaration d'Helsinki (1964) élaborée par l'Association médicale mondiale. Ces principes visent à s'assurer que toute étude respecte la santé, le bien-être, la vie privée et les données personnelles des participants, afin de protéger leur intégrité. Seules les recherches se justifiant par un bénéfice potentiel sur la santé des individus et ne portant pas atteinte à leur intégrité pourront être menées. Dans le cas des interventions avec un groupe contrôle, il est impératif de s'assurer qu'il n'y a pas de risque que le groupe contrôle encoure des préjudices du fait de ne pas avoir reçu l'intervention. Par exemple, si on recrute des individus sur des listes d'attente pour accéder à des jardins partagés afin d'évaluer les bénéfices de ces jardins sur l'alimentation, on ne peut pas contraindre une partie des individus à ne pas avoir accès à ces jardins. Les designs tels que les essais avec permutation séquentielle sont alors à privilégier afin que tous les individus bénéficient du programme à un moment donné. Toute étude doit être au préalable évaluée auprès d'un comité d'éthique de la recherche (Institutional Review Board), qui examinera le protocole de recherche afin de s'assurer qu'il respecte les principes éthiques reconnus. Avant de participer à l'étude, les participants doivent également donner leur consentement éclairé, c'est-à-dire qu'ils doivent être informés des objectifs de l'étude, du protocole, des données collectées, ainsi que de leurs droits et garanties prévues par la loi. Les exigences relatives au consentement éclairé et à la confidentialité varient selon le type d'intervention et le type de données recueillies et sont encadrées par la loi Jardé (décret n²016-1537 du 16 novembre 2016). Le traitement des données collectées est, quant à lui, encadré par le règlement général sur la protection des données (RGPD).

Un facteur important de la réussite d'une intervention est l'implication des participants tout au long de l'étude. Cette implication dépend en grande partie de la posture du chercheur qui se doit d'établir une relation de confiance en favorisant la communication et en étant à l'écoute des participants. L'utilisation de mesures incitatives (en général financières), la mise en place d'un suivi régulier afin de garder contact avec les participants, ou encore l'implication des participants dans la conception de l'étude (co-construction) sont autant de solutions qui peuvent encourager la participation. Il est toutefois important de veiller au respect de la déontologie de la recherche. En particulier, l'incitation financière ne peut pas être utilisée sur des populations fragiles (comme les enfants) ou pour inciter les participants à agir contre leurs principes moraux. Aussi, le suivi ne doit pas enfreindre le respect de la vie privée, etc.

Enfin, la recherche interventionnelle en prévention ou promotion de la santé a une visée d'utilité sociale, son objectif étant d'identifier des actions pouvant favoriser la santé des individus tout en luttant contre les inégalités sociales de santé. Elle peut ainsi être utilisée comme un outil d'aide à la décision auprès des décideurs afin de développer des projets (en général des programmes ou des politiques de santé) favorables à la santé et au bien-être des populations. La co-construction de l'intervention et de l'évaluation avec tous les acteurs concernés, qu'ils soient institutionnels, territoriaux, professionnels ou citoyens, est particulièrement importante pour s'assurer que l'intervention est pertinente et facilement transférable dans le contexte étudié. 


\section{- Adaptation au contexte : l'exemple de l'étude Cash for Nutrition Awareness au Mali}

La conception et/ou la mise en œuvre des études d'impact peuvent nécessiter des ajustements selon le contexte dans lequel elles sont menées. Si l'on reprend l'exemple cité plus haut de l'essai contrôlé randomisé qui a été mené dans la région de Kayes au Mali (Adubra et al., 2019), plusieurs adaptations ont été nécessaires :

- le groupe contrôle : comme dans beaucoup d'autres études interventionnelles, il était éthiquement impossible de disposer d'un groupe contrôle qui ne bénéficierait d'aucune intervention. Il a donc été décidé, en accord avec les opérateurs du programme, que le groupe contrôle bénéficierait d'un paquet minimum d'activités en santé et en nutrition. Afin d'assurer la comparabilité entre les groupes, les groupes d'intervention bénéficiaient également de ce paquet minimum d'activités, en plus des interventions. Du point de vue de la recherche, cette configuration implique que les interventions soient évaluées en comparaison avec un paquet minimum d'activités et non pas en comparaison avec un groupe contrôle absolu (aucune intervention); - la randomisation : la randomisation des individus ou groupes d'individus dans les différents bras de l'intervention est un processus délicat qui nécessite d'être compris et accepté par tous les participants d'une étude d'évaluation. Si la randomisation ne se fait pas en aveugle, le processus de randomisation doit être réalisé en toute transparence pour une meilleure acceptation des populations. Dans l'exemple de l'étude au Mali, où les croyances sont très fortes, il a été nécessaire de rendre la randomisation publique, sous la forme d'un grand événement où étaient invitées les autorités administratives, religieuses et traditionnelles, ainsi que les communautés concernées. La randomisation a alors été réalisée de façon simple et visible, par tirage au sort de boules de couleurs et par une main dite «innocente»;

- le suivi des interventions : la distribution de produits nutritionnels dans un contexte comme le Mali peut poser problème en termes d'évaluation, dans la mesure où les produits distribués sont souvent revendus ou échangés sur les marchés localement. Cette pratique nécessite de mettre en place des vérifications lors des enquêtes évaluatives, comme le fait de contrôler les pots ou sachets vides des produits distribués.

\section{" Théorie de l'intervention et évaluation du processus}

Contrairement aux essais thérapeutiques, où l'efficacité de l'intervention est jugée au regard d'un seul critère, les interventions en santé publique sont relativement complexes. La relation causale entre l'intervention et l'effet sur la santé peut mettre en œuvre une chaîne de mécanismes interagissant entre eux et avec le contexte local. En ce sens, les interventions en prévention et promotion de la santé nutritionnelle s'appuient sur une vision holistique de la santé, car elles visent à agir sur les différents déterminants de la santé, qu'ils soient individuels, sociaux, sociétaux, économiques ou environnementaux. Une évaluation cohérente, utile et explicative doit avant tout s'appuyer sur «la logique» (ou la théorie) de l'intervention, autrement dit sur les hypothèses causales qui la sous-tendent. S'appuyer sur un modèle logique permet d'identifier théoriquement les voies d'impact d'une intervention afin de comprendre comment elle a atteint ou non ses objectifs, en soumettant à l'épreuve des faits les voies d'impact hypothétiques 
identifiées lors de la conception de l'intervention. Une théorie complète de l'intervention doit également contenir une théorie du processus, autrement dit un plan opérationnel précis visant à assurer une mise en œuvre optimale de l'intervention telle qu'elle a été initialement conçue. Lévaluation d'impact et l'évaluation de processus sont indissociables, puisque, si l'impact escompté d'une intervention n'est pas atteint, son évaluation doit être en mesure d'identifier si l'échec provient d'un problème de mise en œuvre ou d'une défaillance dans sa conception. Si c'est la mise en œuvre qui est en cause, il conviendra de recommander des mesures visant à améliorer la qualité de l'intervention, tandis que si c'est la théorie qui est incriminée, c'est la stratégie interventionnelle adoptée qui devra être complètement repensée. La logique sous-jacente à l'intervention doit être pensée dès la conception de l'étude afin de guider l'identification des données qu'il convient de recueillir et la façon dont elles devront être analysées. Par ailleurs, l'effet de l'intervention sur les capacités, croyances et valeurs des individus sera particulièrement important à considérer comme un premier élément de preuve de l'efficacité de l'intervention dans le cas où les impacts sur la santé ne sont pas immédiatement visibles et observables dans le temps de la recherche : on peut alors supposer que ces impacts pourraient se révéler sur le long terme. C'est pourquoi il est souvent pertinent d'engager un dialogue interdisciplinaire (épidémiologie nutritionnelle/santé publique/sciences sociales) et d'utiliser des méthodes d'enquêtes qualitatives (entretiens approfondis, observations, etc.) afin d'approfondir les relations causales testées par l'évaluation quantitative, et d'être en mesure d'expliquer les mécanismes de changement ou l'absence de changement de comportements.

Il est également important de rappeler que la mesure de l'effet d'une intervention de prévention ou promotion de la santé s'insère dans un cadre plus large d'évaluation des incidences économiques, sociales ou encore environnementales de l'intervention pour évaluer sa pertinence. Cette démarche évaluative intervient à toutes les étapes de l'étude afin de vérifier : 1) la pertinence de l'action (i.e. les objectifs répondent aux besoins identifiés), 2) l'efficacité (i.e. les résultats obtenus sont conformes aux objectifs fixés), 3) l'efficience (i.e. l'importance des moyens matériels, humains et financiers mobilisés), et enfin 4) l'évaluation des impacts directs, mais aussi indirects tels que ceux observés sur l'environnement, les acteurs de terrains ou d'autres groupes de personnes qui n'étaient pas ciblés directement par l'intervention.

Pour conclure ce chapitre, les évaluations d'impact sont indispensables pour documenter l'efficacité des interventions et ainsi aider à la prise de décision. Les études expérimentales, de par l'assignation aléatoire des individus (ou randomisation), ont une forte validité interne et sont donc considérées comme la méthode de référence pour évaluer l'impact d'une intervention. Différents designs peuvent être envisagés en fonction de la problématique de recherche, du contexte de l'étude et de la population ciblée. Lorsque la randomisation n'est pas possible pour des raisons pratiques ou éthiques, les designs quasi expérimentaux sont à privilégier afin d'avoir une estimation la moins biaisée possible de l'effet de l'intervention. Même si des ajustements liés aux contextes sont possibles, ces études doivent répondre à de nombreux critères méthodologiques afin de fournir un niveau de preuve suffisant pour imputer les effets observés à l'intervention. 


\section{\ Références}

Adubra L., Le Port A., Kameli Y., Fortin S., Mahamadou T., Marie T., Ruel M. T., Martin-Prével Y., Savy M., 2019. Conditional Cash Transfer and/or Lipid-Based Nutrient Supplement Targeting the First $1000 \mathrm{~d}$ of Life Increased Attendance at Preventive Care Services but Did Not Improve. The American Journal of Clinical Nutrition, 110 (6), 1476-1490. https://doi.org/10.1093/ajen/nqz238

Cornelsen L., Mytton O. T., Adams J., Gasparrini A., Iskander D., Knai C., Petticrew M., Scott C., Smith R., Thompson C., White M., Cummins S., 2017. Change in Non-Alcoholic Beverage Sales Following a 10-Pence Levy on Sugar-Sweetened Beverages within a National Chain of Restaurants in the UK: Interrupted Time Series Analysis of a Natural Experiment. Journal of Epidemiology and Community Health, 71 (11), 1107-1112.

Haute Autorité de santé, 2013. Niveau de preuve et gradation des recommandations de bonne pratique, Saint Denis, Haute Autorité de santé, Service des bonnes pratiques professionnelles, 90 p. [En ligne] https://www.has-sante.fr/upload/docs/application/pdf/2013-06/etat_des_lieux_niveau_ preuve_gradation.pdf (dernière consultation le 07/10/2021).

Hercberg S., Galan P., Preziosi P., Bertrais S., Mennen L., Malvy D., Roussel A.-M., Favier A., Briançon S., 2004. The SU.VI.MAX Study: A Randomized, Placebo-Controlled Trial of the Health Effects of Antioxidant Vitamins and Minerals. Archives of Internal Medicine, 164 (21), 2335-2342. https://doi.org/10.1001/archinte.164.21.2335

De Lorgeril M., Renaud S., Salen P., Monjaud I., Mamelle N., Martin J.-L., Guidollet J., Touboul P., Delaye J., 1994. Mediterranean Alpha-Linolenic Acid-Rich Diet in Secondary Prevention of Coronary Heart Disease. The Lancet, 343 (8911), 1454-1459. https://doi.org 10.1016/s0140-6736(94)92580-1

Ni Mhurchu C., Gorton D., Turley M., Jiang Y., Michie J., Maddison R., Hattie J., 2013. Effects of a Free School Breakfast Programme on Children's Attendance, Academic Achievement and Short-Term Hunger: Results from a Stepped-Wedge, Cluster Randomised Controlled Trial. Journal of Epidemiology and Community Health, 67 (3), 257-264. https://doi.org 10.1136/jech-2012-201540

Tharrey M., Sachs A., Perignon M., Simon C., Mejean C., Litt J., Darmon N., 2020. Improving lifestyles sustainability through community gardening: results and lessons learnt from the JArDinS quasiexperimental study. BMC Public Health, 20 (1), 1798. https://doi.org/10.1186/s12889-020-09836-6

WHO, 1948. Constitution of the World Health Organization, Genève, World Health Organization. [En ligne] https://www.who.int/governance/eb/who_constitution_en.pdf (dernière consultation le 27/08/2021).

WHO, 1986. The Ottawa Charter for Health Promotion, Genève, World Health Organization. [En ligne] https://www.euro.who.int/_data/assets/pdf_file/0004/129532/Ottawa_Charter.pdf (dernière consultation le 27/08/2021). 


\title{
Chapitre 4 \\ L'économie expérimentale : révéler les préférences et les facteurs de décision des individus
}

\author{
Douadia Bougherara ${ }^{a}$, LAURent MUller ${ }^{b}$ et SABrina TeYSSIER ${ }^{b}$
}

a CEE-M, Univ. Montpellier, CNRS, INRAE, Institut Agro, Montpellier, France.

b Université Grenoble Alpes, INRAE, CNRS, Grenoble INP, GAEL, 38000, Grenoble, France.

L'économie expérimentale s'intéresse aux préférences et aux décisions des individus. Elle cherche à objectiver les facteurs causaux qui conduisent les individus à adopter certains comportements plutôt que d'autres. Les protocoles expérimentaux assurent le contrôle des variables de décision et permettent de mesurer l'importance relative des différents facteurs.

Imaginez que l'on vous propose le jeu à pile ou face consistant à lancer une pièce de monnaie. Tant qu'elle sort pile, vous pouvez relancer la pièce. Vous gagnez 1 euro au premier lancé et le gain est doublé à chaque lancé supplémentaire (2 euros au deuxième, 4 euros au troisième, etc.). Si face apparaît, le jeu se termine. Combien seriez-vous prêt à payer pour participer à un tel jeu? La théorie des probabilités est claire : l'espérance de gain étant infinie ${ }^{14}$, vous devriez miser tout ce que vous possédez. Naturellement, cela n'est pas validé empiriquement. C'est à ce paradoxe, dit de «Saint Petersbourg», et mis en évidence par Daniel et Nicholas Bernouilli en 1738, que l'on attribue le premier exemple d'étude en économie expérimentale (Roth, 1995) : ils ont observé les décisions d'individus dans un environnement risqué afin de tester des prédictions théoriques.

À la même époque, dans son ouvrage $A$ Treatise of Human Nature, David Hume introduit l'utilisation d'expériences soignées et rigoureuses pour comprendre l'esprit et le raisonnement humain sur la base des méthodes utilisées en sciences naturelles (Bardsley et al., 2010). À la fin du XIX ${ }^{\mathrm{e}}$ siècle, les pionniers de l'économie néoclassique se servent des résultats de psychologie expérimentale pour fonder l'hypothèse d'utilité marginale décroissante. William Jevons est le premier à avoir publié les résultats d'expériences économiques contrôlées dans une revue scientifique en 1870. Pourtant, peu d'expériences en économie ont été menées par la

14. Lespérance de gain est égale à $\frac{1}{2} \times 1+\frac{1}{2} \times \frac{1}{2} \times 2+\frac{1}{2} \times \frac{1}{2} \times \frac{1}{2} \times 4+\ldots+\frac{1}{2^{n}} \times 2^{n-1}+\ldots=\sum_{n=1}^{\infty} \frac{1}{2}$. 
suite. Seul, Louis Thurstone utilise en 1931 l'expérimentation pour estimer les courbes d'indifférence ${ }^{15}$ d'une personne selon ses choix (hypothétiques) entre différentes options.

La naissance de l'économie expérimentale en tant que discipline économique est couramment attribuée aux retombées de la publication de l'ouvrage de John Von Neumann et Oskar Morgenstern, Theory of Games and Economic Behavior en 1944. La théorie du choix individuel et la nouvelle théorie des comportements stratégiques présentées dans ce livre ont fortement influencé l'économie expérimentale en donnant une nouvelle orientation aux tests expérimentaux. En 1948, Edward Chamberlin, reconnu pour ses travaux en économie industrielle, a conduit une expérience de marché auprès de ses étudiants pour tester le mécanisme de formation des prix. Frederick Mosteller et Philip Nogee conduisent en 1951 la première expérience élicitant des choix réels, i.e. avec des conséquences monétaires réelles, dans un environnement risqué. À la même époque, Merrill Flood et Melvil Dreher réalisent plusieurs expériences visant à étudier les comportements en situation d'interactions stratégiques, i.e. dans des jeux, afin de tester le concept d'équilibre de John Nash. En 1953, Maurice Allais utilise la méthode expérimentale avec des choix hypothétiques et s'intéresse au concept même de rationalité en économie ( «paradoxe d'Allais»). Quelques années plus tard, en 1961, Daniel Ellsberg réalise la première expérience en univers incertain ("paradoxe d'Ellsberg»). Ces premières études expérimentales, bien que publiées dans les revues économiques de premier plan, n’ont pas été considérées comme majeures par la communauté des économistes de l'époque.

La suite du développement de l'économie expérimentale peut être découpée en trois grandes périodes (Serra, 2012). Les années 1960 et 1970 correspondent au démarrage de l'économie expérimentale. Au début des années 1960, Vernon Smith a publié une des expériences les plus influentes pour le développement ultérieur de la discipline. Il a repris le protocole expérimental de Chamberlin en ajoutant de la répétition dans les choix et a testé différentes règles du marché. En 1975, il crée à l'université d'Arizona le premier laboratoire d'économie expérimentale informatisé, l'Economic Science Laboratory, avant de publier en 1976 un article sur la méthodologie expérimentale en économie, qui deviendra une référence (Smith, 1976, repris dans Smith, 1982). L'économie expérimentale se développe en parallèle en Allemagne, notamment avec les travaux de Reinhard Selten, et plusieurs revues économiques généralistes commencent à publier des études expérimentales à partir de la fin des années 1970.

Le véritable décollage de l'économie expérimentale se fait au cours des années 1980 avec une croissance exponentielle des travaux dans le domaine. L'économie expérimentale est dès lors considérée comme une véritable contribution au courant dominant de l'économie. C'est à cette époque que la communauté des expérimentalistes se structure, avec notamment la création en 1986 de l'Economic Science Association ${ }^{16}$, qui est chargée de promouvoir l'économie expérimentale et d'organiser des congrès pour les expérimentalistes. La phase de maturité est

15. Les courbes d'indifférence en économie représentent les préférences des agents économiques et servent à analyser leurs choix dans différents contextes. Ce concept est un des fondements de l'économie néoclassique.

16. https://www.economicscience.org/ (dernière consultation le 30/08/2021). 
considérée atteinte depuis le milieu des années 1990 avec de nombreux travaux acceptés par l'ensemble des revues scientifiques, des numéros spéciaux publiés, le premier Handbook édité en 1995 (Kagel et Roth, 1995), suivi par d'autres, et même un journal dédié, Experimental Economics, créé par Charles Holt en 1998. En 2015, une autre revue est créée, le Journal of the Economic Science Association, pour valider la méthode expérimentale en publiant des études de réplications, de tests de robustesse, des méta-analyses, ainsi que des études utilisant des protocoles validés, même si les résultats sont non significatifs ${ }^{17}$. L'économie expérimentale est pleinement reconnue dans la discipline, avec l'attribution en 2002 du prix Nobel d'économie ${ }^{18}$ à Vernon Smith "pour avoir fait de l'expérience en laboratoire un instrument d'analyse économique empirique, en particulier dans l'étude de différentes structures de marché» et à Daniel Kahneman "pour avoir introduit en sciences économiques des acquis de la recherche en psychologie, en particulier concernant les jugements et les décisions en incertitude ${ }^{19}$. V. Smith et D. Kahneman représentent les deux principaux courants de l'économie expérimentale, celui de l'étude des institutions de marché et celui de l'étude de la rationalité des agents économiques. D'autres prix Nobel d'économie ont depuis récompensé des travaux qui utilisent largement la méthodologie expérimentale : Elinor Oström en économie des organisations en 2009, Alvin Roth en théorie des jeux en 2012 et Richard Thaler en économie comportementale en 2017.

\section{" Démarche méthodologique de l'économie expérimentale}

L'économie expérimentale repose sur les mêmes fondements méthodologiques que l'expérimentation scientifique. À la manière du chercheur qui teste l'introduction de produits chimiques dans des tubes à essai, il s'agit ici en quelque sorte de mettre des individus sous observation et de tester l'effet de chocs exogènes tout en contrôlant l'environnement. Par exemple, si l'on souhaite mesurer l'impact d'un label bio sur les consentements à payer (CAP) pour un produit alimentaire, on comparera une mesure de CAP pour un aliment sans label bio au CAP pour ce même aliment avec label bio. Toute chose égale par ailleurs, la différence de CAP reflétera l'impact du label bio. Quelle que soit la question posée, l'économie expérimentale repose sur des principes méthodologiques précis. Après avoir défini ce qu'est l'économie expérimentale, nous présenterons ses objectifs et les principes que respectent les expériences en économie. Ensuite, nous détaillerons les composantes d'un protocole expérimental et expliquerons ce qu'est un traitement. Puis, nous exposerons le cadre de deux types d'expériences en alimentation et, pour terminer, nous soulignerons quelques points de vigilance à considérer.

17. De telles initiatives visent à lutter contre le «biais de publication», qui décrit la tendance à favoriser la soumission et la publication d'articles donnant des résultats statistiquement significatifs, les travaux aux résultats non significatifs étant «mis au tiroir», car moins faciles à valoriser.

18. Le nom complet de ce prix est : le prix de sciences économiques institué par la Banque de Suède à la mémoire d'Alfred Nobel.

19. https://www.nobelprize.org/prizes/economic-sciences/2002/summary/ (dernière consultation le 30/08/2021). 


\section{Définition}

Une expérience en économie consiste à étudier le comportement d'individus, i.e. les sujets expérimentaux, en reconstituant une situation économique simplifiée dans un environnement contrôlé. Dès l'origine, les expériences en économie se sont portées sur l'étude de trois types de comportements : les décisions individuelles, les décisions avec interactions stratégiques, et les décisions en situation d'échanges impersonnels, i.e. via les institutions de marché. L'étude des comportements alimentaires a jusqu'ici principalement été conduite à travers des expériences portant sur les décisions individuelles. Nous porterons notre attention sur l'étude des décisions individuelles dans la suite de ce chapitre. Dans ce cadre particulier, l'économie expérimentale permet de révéler les préférences des consommateurs en les mettant en situation de choix et aussi de mesurer les impacts de variables explicatives, comme le prix ou l'offre, sur leurs décisions d'achats.

\section{Objectifs}

Léconomie expérimentale a trois objectifs (Roth, 1988) : tester la théorie afin d'identifier des mécanismes non prédits par les modèles existants; produire des faits afin d'identifier des régularités comportementales et ainsi les intégrer dans les modèles théoriques existants ou construire un nouveau modèle théorique. Ces deux objectifs sont interdépendants et s'entretiennent mutuellement. Le dernier objectif est d'aider à la décision en testant les implications de la mise en place d'un nouveau mécanisme organisationnel ou institutionnel. L'économie expérimentale permet ainsi d'évaluer l'impact de la mise en place de politiques publiques diverses comme les politiques d'information (étiquetage, labels, messages) ou l'instauration d'une taxe ou d'une subvention.

\section{Principes}

Léconomie expérimentale respecte quatre principes : le contrôle, la réplication, les incitations financières et la non-tromperie.

L'expérimentateur contrôle l'ensemble des variables : le contrôle des variables d'intérêt et de leurs valeurs lui permet de mesurer l'impact dans des conditions strictement identiques (ceteris paribus) de celles-ci sur les comportements des sujets; le contrôle de variables autres que celles d'intérêt et pouvant influer sur les comportements lui permet de tenir compte de leur influence potentielle. Ce contrôle permet à l'expérimentaliste d'identifier le ou les facteurs qui ont conduit au phénomène observé et ainsi d'identifier l'effet causal du mécanisme étudié. On parle de validité interne des expériences.

Une expérience en économie doit pouvoir être reproduite par d'autres chercheurs. Pour ce faire, le protocole expérimental doit être décrit et explicité exhaustivement. La réplication des expériences avec des différences mineures permet de tester la robustesse des résultats obtenus dans l'expérience initiale.

Une des spécificités des expériences en économie (notamment par rapport aux expériences en psychologie) est l'utilisation de rétributions financières données 
aux sujets expérimentaux en fonction de leurs décisions dans l'expérience. Ainsi, les décisions des sujets dans l'expérience impliquent des conséquences réelles. Les incitations financières ont pour objectif de susciter des comportements réels de la part des sujets et ainsi d'éliciter leurs véritables préférences. Sans incitations financières, les sujets pourraient être tentés de choisir aléatoirement entre plusieurs options ou de prendre des décisions selon des critères indépendants de leurs véritables préférences (biais de désirabilité). Dans des expériences visant à étudier les comportements alimentaires, il est très important que les choix des sujets aient des conséquences réelles comme l'achat réel de produits afin de les inciter à révéler leurs véritables préférences pour les produits proposés. Les incitations financières peuvent être mises en place de deux façons (tous les sujets en sont informés au début de l'expérience) : soit tous les sujets, soit quelques sujets tirés au sort à la fin de l'expérience voient leurs décisions avoir des conséquences réelles. Les sujets peuvent également percevoir une indemnité de participation. Cette indemnité, indépendante des choix (au contraire des rémunérations), est particulièrement utile lorsque la tâche expérimentale génère des pertes et non des gains (choix risqué, achat de bien).

Tromper les sujets ne fait pas partie des pratiques acceptables en économie expérimentale : il n'est pas possible de donner de fausses informations aux sujets. Par exemple, il n'est pas admis de donner des instructions fausses, d'utiliser des complices ou de mentir sur la rémunération. Cette règle garantit que les sujets savent que leurs décisions dans l'expérience auront bien les conséquences réelles décrites par l'expérimentateur au début de l'expérience et assure ainsi la révélation de leurs véritables préférences. L'application de cette règle est stricte afin de prévenir toute contamination des sujets de futures expériences qui pourraient, s'ils pensent que les informations qui leur sont données sont faussées, prendre des décisions qui ne reflètent pas leurs véritables préférences.

\section{Protocole expérimental}

Selon Smith (1982), le protocole expérimental repose sur trois éléments : l'environnement, l'institution et le résultat.

L'environnement de l'expérience correspond à l'ensemble des circonstances initiales de l'expérience. Il s'agit du type de sujets impliqués dans l'expérience et de l'ensemble de leurs caractéristiques dans le contexte de l'expérience, étant donné l'objet de l'étude. Ces critères peuvent être leur situation socio-économique (âge, genre, catégorie professionnelle, revenu...), leur situation géographique (lieu de résidence, lieu de travail...), leur niveau d'études (diplôme, discipline...), mais aussi leurs habitudes alimentaires (repas-type, consommation de viande, plats cuisinés ou non...). Les caractéristiques des sujets dans le contexte de l'expérience incluent les dotations initiales données aux sujets (argent, produit, aucune dotation...) et les technologies auxquelles ils sont confrontés (ordinateur, papier-crayon...).

L'institution correspond aux «règles du jeu» de l'expérience. Ces règles incluent le type de tâche demandée aux sujets (effort, choix de loteries, achat de produits...) et le déroulement de l'expérience (nombre de parties, ordre de présentation des tâches...). Au début de l'expérience, l'expérimentateur donne des instructions aux 
sujets qui décrivent l'institution dans laquelle ils se trouvent. Ces instructions sont données de la même façon à tous les sujets de l'expérience, de sorte que l'ensemble des règles est de connaissance commune. L'expérience commence seulement une fois que tous les sujets ont bien compris ces instructions. L'expérimentateur pose quelques questions de compréhension aux sujets afin de s'assurer que les instructions ont bien été comprises par tous.

Le résultat correspond aux observations des décisions et des choix des sujets dans l'expérience, faites par l'expérimentateur. L'expérimentateur doit s'assurer d'observer l'ensemble des variables d'intérêt qui lui permettent de traiter la question étudiée. Le résultat découle de l'environnement et de l'institution de l'expérience.

\section{Traitements}

Un environnement particulier associé à une institution particulière correspond à un traitement. Le terme traitement vient des essais cliniques en médecine. Pour tester l'effet d'un traitement médical, on soumet une partie de l'échantillon au traitement et on en compare les effets à un groupe non-traité (placebo). Le principe est le même en économie expérimentale. L'expérimentateur modifie l'environnement ou l'institution d'un traitement à l'autre. Afin de contrôler ces changements, il est fortement recommandé de ne procéder qu'à une seule modification à la fois de façon à isoler l'effet du changement. Ainsi, l'expérimentateur est capable de mesurer l'impact causal d'un aspect de l'environnement ou de l'institution sur le résultat. Reprenons l'exemple de la question de recherche sur l'impact du label bio. Imaginons que la question de recherche est l'impact du label bio sur les préférences des consommateurs envers une brique de lait demi-écrémé. Une expérience pertinente consisterait ici à évaluer la valeur monétaire qu'accordent les sujets ${ }^{20}$ à un litre de lait demi-écrémé étiqueté avec un label bio et, dans des conditions ceteris paribus, à un litre d'un lait demi-écrémé non étiqueté bio. La valeur attribuée au label bio est alors obtenue par la différence entre les valeurs accordées aux deux laits (bio et non-bio).

Pour construire son protocole, l'expérimentateur a essentiellement le choix entre deux configurations de comparaisons entre traitements : les comparaisons interindividuelles (between-subjects) ou intra-individuelles (within-subjects).

On parle de comparaisons inter-individuelles quand chaque sujet participe à un seul traitement. Dans notre exemple, un groupe de sujets estime le lait bio et un autre groupe le lait non-bio. Dans ce cas, pour que la comparaison entre les traitements identifie bien l'effet causal du label bio sur le résultat, les sujets assignés aux deux traitements doivent être les plus semblables possible. Il est cependant très difficile de construire deux échantillons parfaitement comparables, même avec l'aide de questionnaires qui précèderaient le recrutement des sujets. Il est alors conventionnel de s'en remettre au hasard en assignant aléatoirement des sujets aux différents traitements. Si la taille de l'échantillon est suffisamment grande, alors les facteurs inobservables qui caractérisent les sujets devraient être uniformément distribués entre les traitements.

20. Voir la section «Deux types d'expériences en alimentation : les expériences de valeurs et les expériences de choix» ci-après. 
Une autre possibilité est d'effectuer des comparaisons intra-individuelles. Chaque sujet participe à plusieurs traitements de manière séquentielle. Ainsi, dans notre exemple, un même sujet estime successivement le lait bio et le lait non bio. La question de la comparabilité des échantillons ne se pose donc plus puisque les mêmes sujets participent aux différents traitements. Néanmoins, un autre problème apparaît : l'effet d'ordre. Il est possible que l'ordre de la tâche impacte les résultats. Les estimations de nos briques de lait peuvent différer selon que le lait bio est apprécié en premier ou en deuxième. L'expérimentateur doit alors s'assurer que l'ordre des traitements n'affecte pas les comportements. Pour contrôler cet effet d'ordre, il est d'usage de proposer les traitements dans tous les ordres possibles, et ce, aléatoirement selon les sujets ${ }^{21}$.

\section{W Les expériences de valeurs et les expériences de choix}

Les expériences portant sur l'étude des décisions individuelles permettent de révéler les préférences des sujets pour différents produits alimentaires avec différents attributs. Les deux grandes catégories d'expériences sont les expériences de valeurs et les expériences de choix.

Dans les expériences de valeurs, l'expérimentateur mesure des dispositions à payer. Une disposition à payer est le montant maximum qu'un individu est prêt à payer pour un produit ou pour un attribut de produit. Pour révéler ces dispositions à payer, les décisions des sujets impliquent des conséquences réelles, comme c'est la règle en économie expérimentale. Ici, la conséquence est l'achat du produit. Pour la vente, il est commun d'utiliser des mécanismes d'enchères comme les enchères de Vickrey ou le mécanisme de Becker, DeGroot et Marshak (BDM) (Lusk et Shogren, 2007 pour une vue d'ensemble de la méthode). Ces mécanismes d'enchères permettent de révéler les véritables préférences des sujets, car il est dans le propre intérêt du participant de proposer un montant égal à sa disposition à payer. Prenons l'exemple du mécanisme BDM et de notre brique de lait bio. Les sujets déclarent le montant maximum qu'ils sont prêts à payer pour la brique. À la fin de l'expérience, l'expérimentateur tire au sort un prix parmi un ensemble de prix définis avant l'expérience. Si le montant maximum que le sujet est prêt à payer pour la brique de lait est inférieur au prix tiré au sort, alors il n'achète pas la brique et ne paie rien. $\mathrm{Si}$, au contraire, le montant déclaré est supérieur au prix tiré au sort, alors le sujet achète la brique et paie le prix tiré au sort. Ainsi, le sujet a intérêt à révéler le véritable montant qu'il est prêt à payer. En effet, s'il surestime sa disposition à payer, il court le risque d'acheter plus cher la brique qu'il ne la valorise réellement; S'il la sous-estime, il court le risque de ne pas acheter la brique à un prix inférieur à sa disposition à payer.

Dans les expériences de choix, les sujets doivent choisir entre des produits qui diffèrent selon leurs attributs et aussi selon leur prix. Cette tâche est plus naturelle que celle des expériences de valeurs. Il est en effet plus aisé de choisir son produit préféré parmi un ensemble de produits à prix affichés (comme nous le faisons tous dans les linéaires de

21. Il est parfois possible qu'un ordre particulier soit naturel (apport d'information, situation simple avant situation complexe...) et, dans ce cas, un seul ordre est proposé. 
grandes surfaces) plutôt que d'estimer un montant seuil pour lequel vous seriez prêt à acheter le produit. Néanmoins, pour révéler une disposition à payer, cette méthode requiert de faire choisir les sujets pour un grand nombre de combinaisons de prix. Une analyse statistique de l'ensemble des choix permet alors de déterminer la disposition à payer du sujet pour un attribut particulier (Louviere et al., 2000). Dans notre exemple, une brique de lait bio et une brique de lait non bio seraient proposées au sujet. Ce dernier choisirait son option préférée pour différentes combinaisons de prix. À la fin de l'expérience, l'expérimentateur tire au sort une situation de choix et le choix du sujet est appliqué : s'il a choisi une brique de lait, il l'achète au prix indiqué.

Les expériences de valeurs et les expériences de choix sont utilisées pour mesurer la disposition à payer des sujets pour un produit alimentaire ou pour un attribut particulier. Elles peuvent également être utilisées pour étudier la diète des individus (ou panier de produits), comme nous le verrons dans l'exemple en fin de chapitre (voir la section «Un cas d'application : l'impact de taxes et subventions sur la composition des paniers alimentaires »).

\section{Points de vigilance et limites}

Une des limites de l'économie expérimentale, importante pour l'étude des pratiques et consommations alimentaires, est la question de la validité externe des résultats expérimentaux : est-ce que les décisions des individus lors d'expériences reflètent bien les comportements dans des environnements économiques réels ? Steven Levitt et John List (2007) critiquent le caractère artificiel des expériences en laboratoire, qui peut conduire à des résultats différents de ceux qui seraient obtenus dans une situation économique «in vivo». Le premier point qu'ils soulèvent est que les sujets d'une expérience savent qu'ils sont observés par les expérimentateurs et, en conséquence, adaptent leur comportement de façon à les satisfaire. Deuxièmement, ils pointent l'importance du contexte, qui est différent dans l'expérience par rapport à l'environnement économique réel. Troisièmement, ils soulignent que les individus choisissent librement de participer à une expérience et ce processus de sélection peut biaiser les décisions dans l'expérience. Enfin, ils questionnent le rôle des incitations monétaires qui ne sont pas nécessairement de taille comparable entre l'expérience et l'environnement économique réel.

Pour remédier au problème de la validité externe, des efforts ont été consentis pour rendre les expériences plus écologiques (Harrison et List, 2004). Des expériences plus contextualisées ont vu le jour, notamment les expériences de terrain contrôlées, dans lesquelles les sujets appartiennent à la population cible et l'environnement reflète la question étudiée. Des études portant sur l'observation des comportements dans des expériences naturelles se sont également développées. Dans ce dernier type d'expériences, les sujets réalisent une tâche dans leur environnement habituel, sans savoir qu'ils font partie d'une expérience et sans intervention de la part de l'expérimentateur. Celui-ci observe simplement les décisions dans différents traitements qui ont été mis en place naturellement par les décideurs publics ou des acteurs privés. Il est néanmoins de plus en plus fréquent que les décideurs publics choisissent la façon de mettre en place certaines mesures en collaboration avec des chercheurs afin de 
constituer une expérience naturelle qui génère des effets de traitements qui peuvent être testés ${ }^{22}$. Si l'écologisation des expériences améliore la généralisation des résultats (validité externe), elle diminue souvent le contrôle des variables explicatives et donc la capacité de l'expérimentateur à inférer des causalités (validité interne).

\section{W Aspects éthiques et juridiques}

En France, les laboratoires d'économie expérimentale dépendent de laboratoires de recherche, souvent des unités mixtes de recherche (UMR), associés à l'université et au CNRS ou à l'INRAE. Les incitations financières données aux sujets impliquent de pouvoir les rétribuer en espèces. Pour ce faire, les laboratoires en économie expérimentale sont généralement équipés d'une régie d'avance.

Une des conditions de base de l'économie expérimentale est la non-tromperie. L'expérimentateur doit s'assurer que les sujets qui vont participer à son expérience sont conscients de cette règle. En effet, le problème se pose quand des sujets participent également à des études dans d'autres disciplines qui utilisent la tromperie (comme la psychologie).

Comme toute étude impliquant des individus, l'économie expérimentale pose la question de la confidentialité des données. L'anonymat est garanti aux sujets dans les expériences en économie. D'une part, les sujets se voient attribuer un code et les décisions qu'ils prennent durant l'expérience ne peuvent en aucun cas être associées à leur identité, même par l'expérimentateur lui-même. Dans les fichiers contenant les données de l'expérience, l'identité des sujets n'est jamais mentionnée. D'autre part, les sujets ne connaissent pas l'identité des autres sujets participant à l'expérience, sauf si c'est l'objet même de l'étude. Comme les expériences génèrent des données personnelles, elles doivent être conformes au règlement général de protection des données (RGPD).

Par ailleurs, de plus en plus d'instances, comme les revues scientifiques ou les agences de financement de la recherche, demandent que les protocoles expérimentaux soient validés par des comités d'éthiques. Ces comités d'éthiques sont généralement localement créés par les universités ou des fondations de recherche publique et sont composés de chercheurs de différentes disciplines utilisant la méthodologie expérimentale avec des humains. L'expérimentateur doit soumettre son protocole expérimental au comité, qui vérifie que le protocole ne pose pas de problème éthique.

Enfin, certains expérimentalistes commencent à enregistrer leur protocole, ainsi que l'objectif visé de l'étude sur des plateformes en ligne. Cette procédure sert à garantir que l'analyse des résultats est conforme à la problématique étudiée et ainsi à éviter que le chercheur prétende avoir cherché à répondre à une problématique différente sur la base des résultats obtenus.

22. Par exemple, des chercheurs ont été associés à la mise en œuvre d'une campagne de levée de fonds (par courrier) par l'université de Floride pour doter d'ordinateurs un centre de recherche. Les chercheurs ont pu proposer plusieurs versions de la campagne, envoyées de manière aléatoire, pour tester l'impact du niveau de financement déjà acquis par ailleurs sur le montant des contributions. Les résultats indiquent qu'une augmentation du financement déjà acquis de 10 à $67 \%$ a multiplié par six les contributions (List et Lucking-Reiley, 2002). 


\section{W L'interdisciplinarité de la méthode expérimentale}

L'économie expérimentale peut contribuer à une approche holistique de l'alimentation et des comportements alimentaires. En effet, la méthode expérimentale est utilisée dans d'autres disciplines, notamment en sciences de l'alimentation (analyse sensorielle et sciences de la nutrition). Les études expérimentales en sciences de l'alimentation portent beaucoup sur l'analyse sensorielle et les préférences des sujets au regard des différents attributs sensoriels des produits alimentaires. Les expériences en économie complètent ces études en ajoutant la dimension économique et l'estimation de la disponibilité à payer des sujets pour les produits alimentaires en fonction de leurs attributs (Lange et al., 2002).

Plus généralement, les comportements alimentaires sont étudiés expérimentalement par des chercheurs de différentes disciplines, comme les neurosciences, la psychologie, le marketing, ou la sociologie. La figure 4.1 présente les connexions entre l'économie expérimentale et les autres disciplines pour étudier les comportements alimentaires.

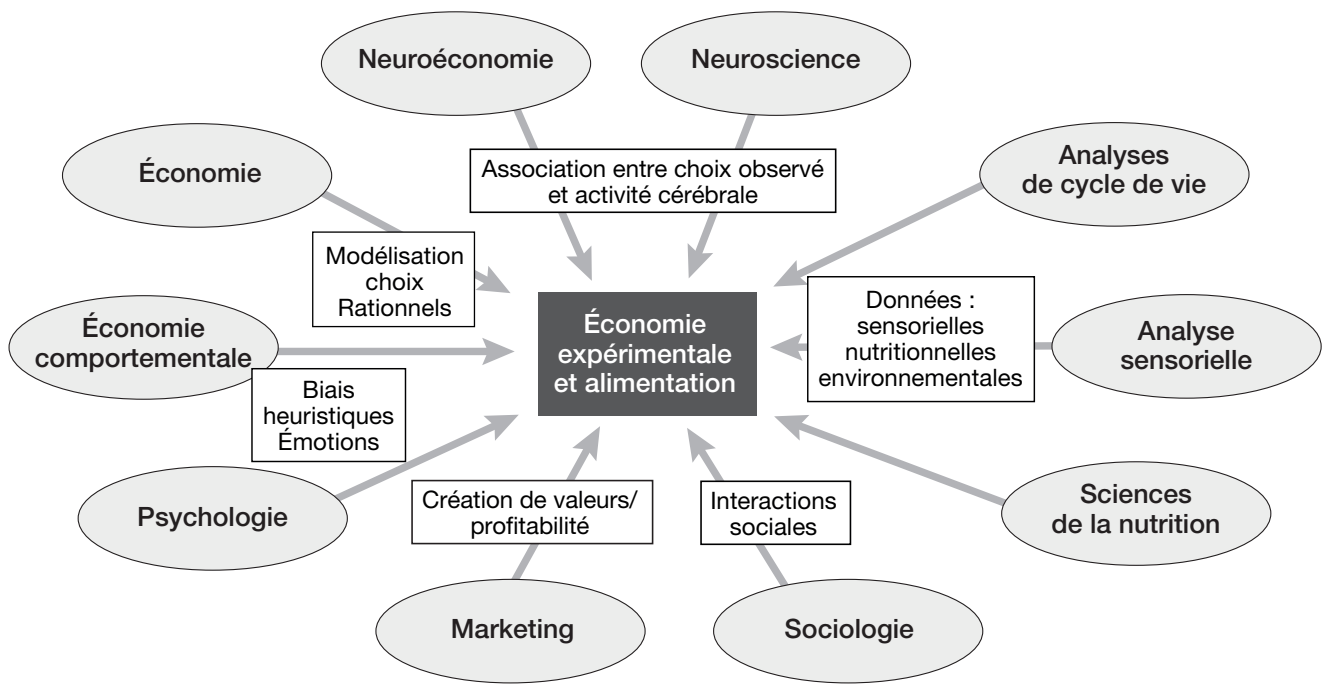

Figure 4.1. Léconomie expérimentale et ses emprunts aux autres disciplines.

\section{W Un cas d'application : incitations financières et panier alimentaire}

Afin de présenter la pratique concrète de la méthode, nous nous appuyons sur une étude expérimentale, dont l'objectif était d'évaluer l'impact d'une politique de taxation des produits «malsains» et de subvention des fruits et légumes et des produits «sains» sur la qualité nutritionnelle de la diète des sujets et sur le prix payé. Cette étude a été conduite pour répondre aux enjeux de croissance de l'obésité en France. Elle a été menée par Laurent Muller, Anne Lacroix, Jayson Lusk et Bernard Ruffieux (Muller et al., 2017). 
Cette étude respecte les principes de l'économie expérimentale (le contrôle, la réplication, les incitations financières, la non-tromperie). Nous présentons concrètement le protocole expérimental choisi, incluant l'environnement, l'institution, le résultat, le type de traitements et les incitations financières, ainsi que leur mise en ouvre.

\section{Environnement}

Létude portait sur les choix alimentaires de femmes de 20 à 50 ans appartenant au premier décile de revenus, pour lequel on sait que l'obésité a la plus forte prévalence. Le recrutement s'est donc fait sur ces critères. Un groupe de contrôle a été ajouté, incluant des femmes de 20 à 50 ans appartenant aux déciles supérieurs de revenus. Au total, 160 femmes âgées de 20 à 52 ans ont participé à l'expérience, dont 103 à Grenoble et 57 à Lyon. Le revenu moyen était de $572 €$ pour les femmes appartenant au premier décile de revenus et de $1459 €$ pour les autres. 21 sessions expérimentales ont été conduites, de deux heures environ. Le recrutement des sujets a été effectué auprès d'une société de recrutement, mais aussi de centres de santé de Grenoble, du Secours populaire et du Secours catholique de Lyon et d'épiceries solidaires. La figure 4.2 présente un exemple de flyer distribué à Lyon.

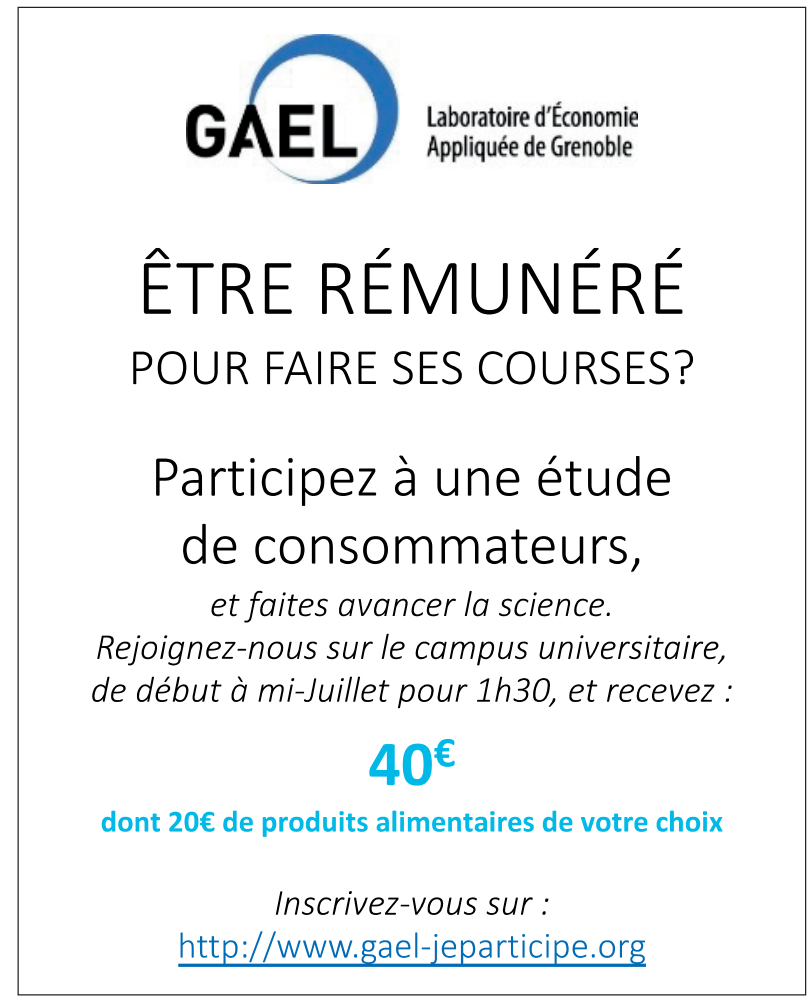

NE PAS JETER SUR LA VOIE PUBLIQUE

Université Grenoble Alpes - UMR GAEL - CS 40700 - 38058 Grenoble CEDEX 9 Imprimé par nos soins

Figure 4.2. Flyer distribué pour le recrutement de volontaires. 


\section{Institution ou règles du jeu}

Au début de l'expérience, l'expérimentateur a présenté les instructions aux participantes. La tâche qu'elles devaient réaliser leur a été expliquée. Dans cette expérience, il s'agissait de composer une journée alimentaire en choisissant les produits alimentaires de la journée du lendemain parmi ceux proposés en fonction de leurs prix. Les choix se faisaient sur un ordinateur. Au total, 180 produits étaient disponibles. Ces produits étaient proposés à l'écran sous forme d'une arborescence facile à manipuler. Les produits étaient classés en catégories de produits, selon leurs compositions nutritionnelles : fruits et légumes, produits «sains» et produits «malsains». Il s'agissait des produits d'entrée de gamme d'un supermarché français. La figure 4.3 montre un exemple de présentation des produits à l'écran.

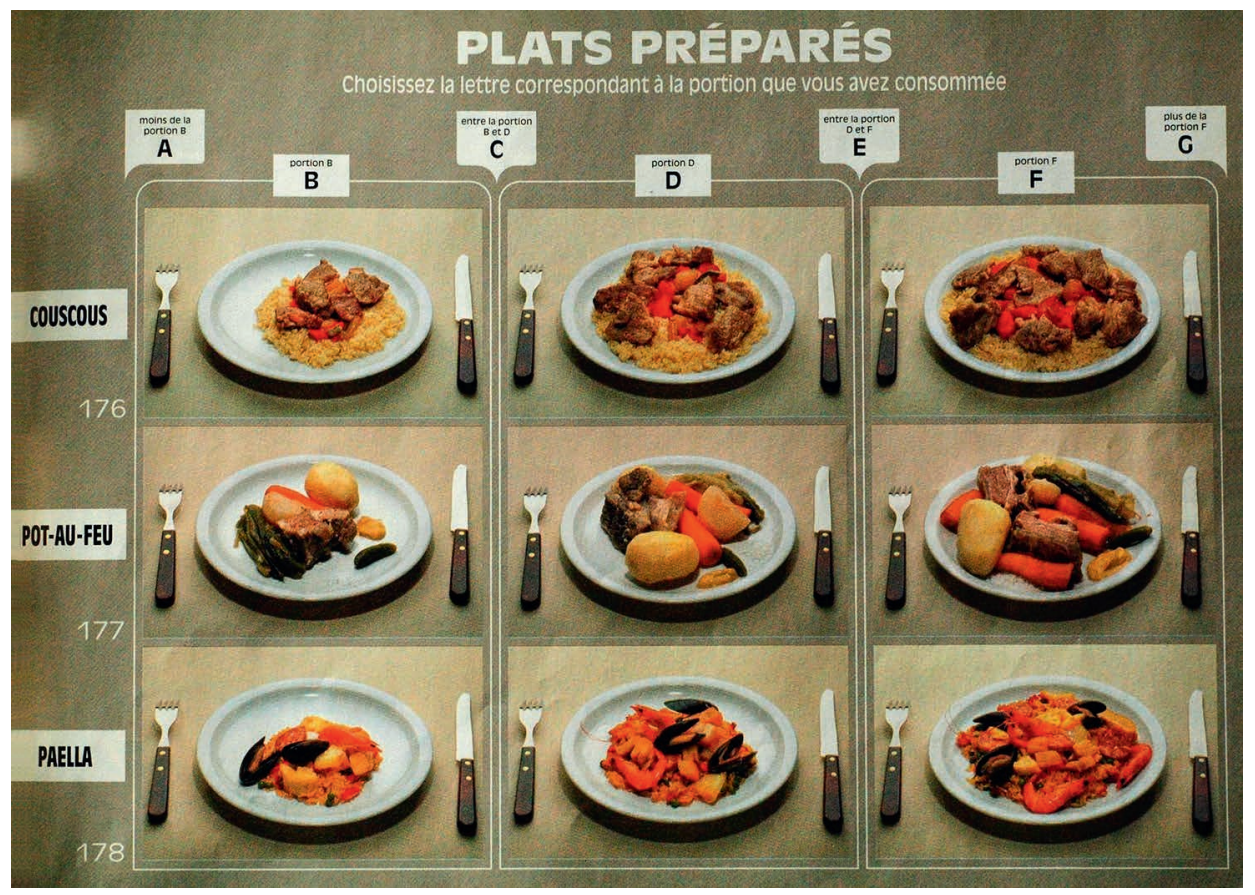

Figure 4.3. Exemple de produits (portions de plats) proposés aux enquêtées.

Avant la tâche, les sujets devaient déclarer leurs caractéristiques sociodémographiques ainsi qu'un rappel des 24 heures, c'est-à-dire tous les produits alimentaires consommés la veille. Après la tâche, elles devaient répondre à un questionnaire de fréquence de consommation et à un questionnaire de santé.

\section{Résultat}

Dans cette expérience, l'expérimentateur observait la qualité nutritionnelle des choix des sujets et le montant dépensé associé aux produits alimentaires choisis. 


\section{Type de traitements}

Le protocole s'appuyait sur des comparaisons intra-individuelles (within-subjects). Les sujets participaient à plusieurs phases de manière séquentielle dans l'expérience. Ils devaient choisir les produits alimentaires pour le lendemain, dont les prix affichés étaient les prix en ligne du supermarché considéré (phase 1); une baisse des prix de $30 \%$ pour les fruits et légumes (phase 2); une baisse des prix de $30 \%$ pour les produits «sains» et une hausse des prix de $30 \%$ pour les produits «malsains» (phase 3). La comparaison des décisions - i.e. la qualité nutritionnelle des produits choisis et le montant dépensé - prises par les sujets en phase 2 et en phase 1 donnent l'impact de la mise en place d'une politique de subvention visant à réduire de $30 \%$ le prix des fruits et légumes. La comparaison des décisions entre la phase 1 et la phase 3 donne l'impact de la mise en place d'une politique de subvention et de taxe avec une subvention des produits «sains» et une taxe sur les produits «malsains».

\section{Incitations financières}

Au début de l'expérience, les sujets recevaient une indemnité forfaitaire en liquide. Leurs décisions lors des trois phases avaient des conséquences réelles. À la fin de l'expérience, une de ces trois phases était tirée au sort par l'expérimentateur et les sujets de la session achetaient les produits qu'ils avaient choisis, aux prix affichés lors de la phase tirée au sort. Les sujets repartaient avec les produits qu'ils avaient achetés. Le montant qu'ils recevaient au début de l'expérience était calculé de façon à ce que chaque sujet reparte avec un montant en liquide positif. La figure 4.4 montre les produits alimentaires prêts pour la vente aux sujets.

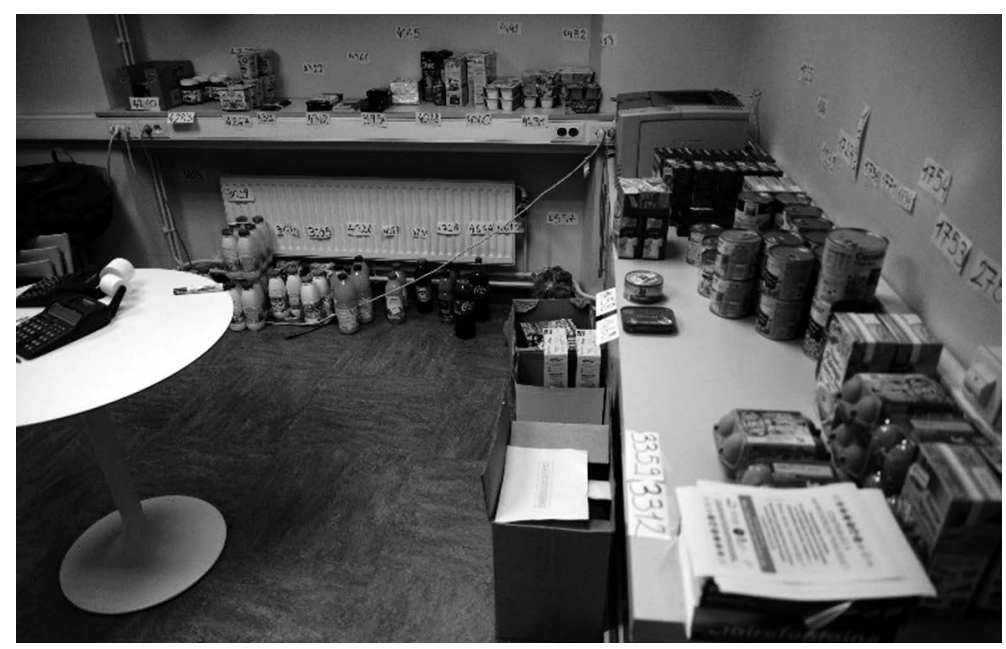

Figure 4.4. Produits alimentaires pour la vente.

Cette expérience a permis de mettre en évidence un effet «structure de consommation» : les femmes à faibles revenus consomment plus de produits «malsains» et moins de produits «sains». En conséquence, leurs choix les conduisent à moins bénéficier d'une subvention des produits «sains» et à subir une plus forte taxation globale de leur 
consommation. Un effet « réactivité à la politique » est également observé : les femmes à faibles revenus sont moins réactives aux politiques. Cette moindre sensibilité aux changements de prix peut s'expliquer par des effets comportementaux tels que les habitudes de consommation. D'après les résultats de l'expérience, ce type de politique subvention/taxe est donc doublement régressif d'un point de vue fiscal.

\section{- Adaptation de la méthode au contexte}

L'exemple d'expérience présenté à la section précédente a été choisi également pour montrer l'importance d'adapter les protocoles expérimentaux au contexte de l'étude. Une des difficultés de cette expérience touchait au public étudié. En effet, certains sujets n'étaient pas habitués aux ordinateurs et pouvaient paniquer lors des phases de décisions. Aussi, plusieurs personnes aux côtés de l'expérimentateur étaient présentes pour les accompagner. Cette différence de traitement entre l'échantillon du $1^{\mathrm{er}}$ décile et l'échantillon témoin compromet le principe strict du ceteris paribus en générant un biais de désirabilité ${ }^{23}$ plus fort pour les sujets assistés. Cela peut fragiliser les conclusions si ce biais n'est pas pris en compte dans l'interprétation des résultats. Plus généralement, l'expérimentateur doit s'adapter au type de sujets présents dans l'expérience pour s'assurer, avant le début de l'expérience, de la bonne compréhension des instructions et de l'attribution du paiement ou de l'achat des produits. L'expérimentateur doit aussi adapter le matériel de l'expérience en évitant par exemple d'utiliser des tâches sur ordinateur.

Les bonnes pratiques de l'économie expérimentale sont générales et doivent être respectées. Néanmoins, l'existence de différents types d'expériences décrits précédemment (Harrison et List, 2004) témoigne déjà de l'adaptabilité de l'économie expérimentale au contexte étudié. Cette adaptabilité est même plus forte car chaque expérience est conçue par l'expérimentateur pour étudier un contexte particulier et se trouve, de fait, adaptée à ce contexte. L'expérimentateur fait preuve d'inventivité afin de créer le protocole expérimental le plus adapté au questionnement de son étude. C'est particulièrement vrai de l'application de la méthode aux questions de consommation alimentaire; le produit pouvant impliquer pour le chercheur des contraintes liées à son caractère périssable (logistique), la gestion de préférences et interdits alimentaires (allergies, positions philosophiques ou religieuses), et l'adaptation à un large public plus ou moins à l'aise avec les instructions associées aux protocoles.

\section{" Références}

Bardsley N., Cubitt R., Loomes G., Moffatt P., Starmer C., Sugden R., 2010. Experimental economics: Rethinking the rules, Princeton, Princeton University Press, $384 \mathrm{p}$.

Harrison G. W., List J. A., 2004. Field Experiments. Journal of Economic Literature, 42 (4), 10091055. [En ligne] https://www.jstor.org/stable/3594915 (dernière consultation le 31/08/2021).

23. Volonté consciente ou inconsciente de se comporter de manière à donner une bonne image de soi à l'expérimentateur. 
Kagel J. H., Roth A. E. (éd.), 1995. The handbook of experimental economics, Princeton, Princeton University Press, $744 \mathrm{p}$.

Lange C., Martin C., Chabanet C., Combris P., Issanchou S., 2002. Impact of the information provided to consumers on their willingness to pay for Champagne: comparison with hedonic scores? Food Quality and Preference, 13 (7-8), 597-608. https://doi.org/10.1016/S0950-3293(02)00059-9

Levitt S. D., List J. A., 2007. What do Laboratory Experiments Measuring Social Preferences Reveal about the Real World? Journal of Economic Perspectives, 21 (2), 153-174.

https://doi.org/10.1257/jep.21.2.153

List J. A., Lucking-Reiley D., 2002. The Effects of Seed Money and Refunds on Charitable Giving: Experimental Evidence from a University Capital Campaign. Journal of Political Economy, 110 (1), 215-233. https://doi.org/10.1086/324392

Louviere J. J., Hensher D. A., Swait J. D., 2000. Stated choice methods: analysis and application, Cambridge, Cambridge University Press, 402 p.

Lusk J. L., Shogren J. F., 2007. Experimental Auctions: Methods and Applications in Economics and Marketing Research, Cambridge, Cambridge University Press, 318 p.

Muller L., Lacroix A., Lusk J. L., Ruffieux B., 2017. Distributional Impacts of Fat Taxes and Thin Subsidies. The Economic Journal, 127 (604), 2066-2092. https://doi.org/10.1111/ecoj.12357.

Roth A. E. 1988. Laboratory Experimentation in Economics: A Methodological Overview. Economic Journal, 98 (393), 974-1031. [En ligne] https:/www.jstor.org/stable/2233717 (dernière consultation le 31/08/2021).

Roth A. E., 1995. Introduction to experimental economics, in Kagel J. H., Roth A. E. (éd.), The Handbook of Experimental Economics, Princeton, Princeton University Press, 3-109.

Serra D., 2012. Un aperçu historique de l'économie expérimentale : des origines aux évolutions récentes. Revue d'économie politique, 122 (5), 749-786. https://doi.org/10.3917/redp.225.0749

Smith V. L., 1976. Experimental economics: induced value theory. American Economic Review, 66 (2), 274-279. [En ligne] https://www.jstor.org/stable/1817233 (dernière consultation le $31 / 08 / 2021)$.

Smith V. L., 1982. Microeconomic systems as an experimental science. American Economic Review, 72 (5), 923-955. [En ligne] https://www.jstor.org/stable/1812014 (dernière consultation le $31 / 08 / 2021)$. 



\title{
Chapitre 5 \\ La mesure du bien-être alimentaire subjectif : saisir comment les mangeurs évaluent leur alimentation
}

\author{
CHRISTOPHE SERRA-MALLOL ${ }^{a}$ ET MILA LEBRUN ${ }^{b}$ \\ ${ }^{a}$ Université de Toulouse Jean Jaurès - ISTHIA, CERTOP - CNRS, France. \\ ${ }^{\mathrm{b}}$ CIRAD, UMR MoISA, F-34398, Montpellier, France; MoISA, université Montpellier, CIRAD, CIHEAM- \\ IAMM, INRAE, Institut Agro, IRD, Montpellier, France.
}

Le bien-être que les mangeurs eux-mêmes associent à leur alimentation peut être évalué à partir de la façon dont ils vivent, sur une période de temps donnée, les différentes situations qui y sont associées. Une telle évaluation met en lumière la diversité des critères qu'ils mobilisent pour l'appréciation de leur bien-être, en lien avec les multiples dimensions de l'alimentation. Elle permet également de resituer la place que l'alimentation occupe dans le bien-être en général.

Les recherches contemporaines sur le bien-être alimentaire trouvent leurs origines dans des questionnements philosophiques très anciens, impossibles à détailler ici. Retenons cependant qu'au-delà des difficultés à s'accorder sur une définition de la notion, ce que l'on appelle aujourd'hui bonheur a longtemps correspondu, de l'Antiquité au $\mathrm{XVIII}^{\mathrm{e}}$ siècle, à une vertu liée à la sagesse, avant d'être rapproché du plaisir à partir du siècle des Lumières, puis du bien-être plus récemment (Diener, 1984). Ce n'est que durant la seconde moitié du $\mathrm{xx}^{\mathrm{e}}$ siècle que le bien-être a été défini comme un objet de recherches empiriques et systématiques. L'objet a été depuis repris par l'économie et la psychologie de type «positive», et accompagne la remise en cause de l'idée de développement purement matériel et quantitatif. Dans ce texte, nous entendons par bonheur un état durable, agréable et équilibré de plénitude spirituelle et physique, en prenant en compte à la fois sa dimension hédonique (la satisfaction par rapport aux émotions vécues, soit le bien-être subjectif, en matière alimentaire) et sa dimension eudémonique (le contentement par rapport à sa vie et la place de l'alimentation au sens large). Quand nous évoquerons dans le texte la satisfaction ou le bien-être, ce sera en référence à cette seule première dimension (hédonique) du bonheur.

Quelles doivent être les conditions de vie au quotidien pour être heureux, quels en sont les déterminants? En quoi la satisfaction ou le bien-être tirés des actions quotidiennes influent-ils sur la satisfaction générale de la vie? Ces questions font 
désormais l'objet de méthodologies et d'outils de recherche des sciences sociales, et s'appliquent également à l'alimentation, même si l'intérêt spécifique porté au «bienêtre alimentaire» est plus récent, et sa méthodologie bien souvent une adaptation d'outils de mesure du bien-être global.

Dans ce chapitre, qui vise à présenter et mettre en perspective les différentes méthodes d'évaluation du bien-être alimentaire, nous faisons le choix méthodologique de privilégier son autoperception par la personne enquêtée, pour nous centrer sur le bien-être alimentaire subjectif et ses différentes méthodes de mesure.

\section{" Le bien-être comme objet de recherche}

Le bien-être peut être caractérisé par un état lié à différents facteurs plus ou moins extérieurs à l'être humain : santé, réussite sociale ou économique, plaisir, réalisation de soi, harmonie intérieure et avec autrui. Il est donc à distinguer du strict plaisir comme sensation, et du bonheur comme état. Cette notion de bien-être est utilisée aujourd'hui dans de nombreux domaines, comme l'éducation ou la santé, ou encore dans les entreprises.

L'économie du bonheur est une branche de l'économie s'appuyant notamment sur les travaux fondateurs d'Easterlin (1974), qui s'intéresse à la définition et à la mesure «objective» du bien-être social. Les travaux en économie et en sciences politiques sur la qualité de vie se sont multipliés depuis les années 1970 à partir d'indicateurs socio-économiques, comme l'indice de développement humain (IDH) ou ceux de l'Organisation de coopération et de développement économiques (OCDE) ${ }^{24}$. Dans cette acception, le bien-être ou la qualité de vie dépendent dans une large mesure du pouvoir d'achat, mais aussi des situations rencontrées dans les domaines du libéralisme économique et politique, et de la santé. En psychologie et en médecine, la qualité de vie est le jugement que porte une personne sur divers aspects de son bien-être physique, social et psychologique, et peut être évaluée par des échelles standardisées (Quality of well being $[Q W B]$, Nottingham Health Profile [NHP], Sickness impact profile [SIP], etc.).

Des travaux récents pour mettre en place des indices de croissance révélant mieux le bien-être que les seuls produit intérieur brut (PIB) et produit national brut (PNB) ont défini le bien-être comme «un phénomène complexe, pluridimensionnel» (Stiglitz et al., 2009a et 2009b), et le recours aux mesures du bien-être subjectif y est fortement prescrit. Toutefois, la dimension culturelle constitue souvent la part d'ombre de l'économie du bien-être, rendant essentielle la prise en compte

24. Créé en 1990 et adopté par le programme des Nations unies pour le développement, l'IDH regroupe plusieurs indicateurs de développement humain (PIB par habitant, espérance de vie, niveau d'instruction moyen des adultes, etc.). Il permet de classer les pays selon des niveaux de développement au-delà de critères strictement économiques. L'OCDE est une organisation internationale fondée en 1948, qui regroupe des pays membres autour de principes comme le gouvernement démocratique et l'économie de marché. L'OCDE publie de nombreux rapports de bilan et de prospective sur le développement économique ou sur les effets des politiques publiques, et produit des indicateurs économiques et sociaux, et de mesure du bien-être subjectif comme le Better Life Index, qui intègre le bien-être subjectif parmi une dizaine de dimensions macrosociales (revenus, logement, éducation, environnement, etc.). 
du contexte culturel local. Les économistes et psychologues qui étudient de façon scientifique la question du bonheur aujourd'hui ont proposé le terme de subjective well-being, auto-évaluation que les individus font de leur vie (Diener, 1984). Les composantes du bien-être subjectif comprennent à la fois une composante cognitive, la life satisfaction, c'est-à-dire le jugement global d'un individu sur sa propre vie, la satisfaction liée aux domaines importants de la vie, mais également la prévalence d'affects positifs et un niveau faible d'affects négatifs dans les activités au quotidien. L'autoévaluation d'émotions ressenties à l'occasion d'épisodes restreints s'avère moins soumise à des biais heuristiques que celle basée sur des longues périodes de temps que les individus ont tendance à relier à leurs croyances et idéologies générales. Cette définition fait des individus les meilleurs juges pour définir leur propre bonheur, loin donc des conceptions antiques et classiques élitistes du bonheur.

Si les individus diffèrent dans leur niveau de bien-être général, l'expérience affective de chaque personne détermine le niveau global de satisfaction, bien que variant substantiellement au cours d'une journée en fonction des activités dans lesquelles elle est engagée et du contexte social dans lequel elle se situe. Le niveau de bien-être est fonction de la satisfaction tirée des différentes activités elles-mêmes : le bonheur individuel pour un jour donné peut être ainsi défini par la moyenne des affects ressentis au cours des différentes activités compte tenu de leur durée relative, en général comme pour l'alimentation. De nombreux travaux montrent l'importance des relations et interactions sociales dans le niveau de bien-être subjectif.

Plusieurs méthodes peuvent être utilisées pour déterminer le niveau de bonheur d'un individu. On peut d'abord poser à un individu une ou plusieurs questions sur son niveau de bonheur général (du type «dans l'ensemble, êtes-vous satisfait de votre vie ?»); il s'agit des méthodes pour déterminer un niveau de life satisfaction comme la Satisfaction With Life Scale (SWLS) à partir de sept questions (Diener et al., 1985). On peut également demander d'une façon générale comment un individu apprécie une activité donnée; c'est la méthode employée par les premières études américaines et européennes sur l'utilisation de son temps, comme les eurobaromètres.

On peut ensuite évaluer le niveau de satisfaction tirée d'une activité en temps réel, en utilisant des méthodes de recueil instantané de l'information, comme la méthode dite Experience Sampling Method (ESM). Il s'agit là sans doute de l'expérience idéale pour évaluer les affects ressentis au cours d'une activité donnée, mais les coûts élevés de sa mise en œuvre et l'ennui qu'elle provoque chez le répondant, appelé à évaluer ses affects de façon aléatoire tout au long d'une journée ou d'une semaine, la rendent difficile à appliquer sur de larges échantillons. Pour éviter de ne pas reporter d'événements dont la fréquence est faible au niveau individuel, on peut aussi recourir à la méthode dite Event Reconstruction Method (ERM), qui recueille chez les personnes interrogées le ressenti sur le plus récent épisode d'une suite d'activités, y compris les plus rares. Mais le risque, opposé au précédent, est que la mémorisation d'événements peu fréquents, et donc reculés dans le temps, subisse de forts biais cognitifs.

Un bon compromis est la méthode développée par Daniel Kahneman et son équipe, qui vise à recueillir des données rétrospectives récentes (en général, du jour précédent) sur les affects des répondants durant leurs différentes activités quotidiennes, y compris les activités touchant à l'alimentation : Day Reconstruction Method ou 
DRM (Kahneman et al., 2004). Le protocole du DRM repose sur un questionnaire constitué de plusieurs cahiers. Les personnes interrogées doivent d'abord répondre à une série de questions portant sur leur satisfaction en général. Puis, il leur est demandé de reconstruire le planning des épisodes de leur journée de la veille, dont les activités alimentaires au sens général (depuis la production ou la disponibilité des ressources jusqu'à leur consommation, en passant par leur préparation), en différenciant chaque épisode en fonction de l'activité réalisée. Chaque épisode est caractérisé temporellement par son heure de début et son heure de fin, les épisodes pouvant être avec activité «focale» (par exemple «manger») ou avec «multi-activités» (par exemple «regarder la TV» et «discuter»), avec la subjectivité possible du choix d'une activité comme «principale». Les personnes enquêtées doivent ensuite répondre à des questions portant sur le lieu où s'est déroulé l'épisode, si elles étaient seules ou non, si elles interagissaient verbalement avec quelqu'un et qui était cette ou ces personnes, et sur les émotions qu'elles ont éprouvées pendant cet épisode en notant sur une échelle de Likert une liste de dix affects, à la fois positifs et négatifs. Enfin, elles doivent répondre à des questions sur les joies et les peines éprouvées à propos d'une liste d'activités (dont alimentaires) et sur leur perception de leur propre santé. Le questionnaire peut être soumis aux répondants individuellement ou en groupe (chacun le remplissant individuellement), et sa durée moyenne est de 45 à 60 minutes.

Des enquêtes menées pour vérifier la fiabilité du $D R M$ ont montré que les questions sur la satisfaction de sa vie en général sont soumises à l'influence des événements quotidiens ou d'une information incomplète. En revanche, les évaluations affectives portant sur les épisodes quotidiens sont moins soumises à ces biais cognitifs, et ont l'avantage d'agréger différentes activités et leurs affects liés pour un jour donné. Enfin, des enquêtes comparatives ont montré qu'il existait plus de différences intraculturelles (entre groupes d'âge et de sexe) qu'interculturelles.

\section{" Les mesures du bien-être alimentaire}

Comme pour évaluer la satisfaction de la vie ou le bien-être en général, l'évaluation de la satisfaction liée à l'alimentation peut comprendre deux types de mesure : une mesure de nature «objective» (à partir d'indicateurs ad hoc (par exemple des critères individuels et sociaux, tels que le montant consacré à l'alimentation et sa part relative aux revenus, l'équipement culinaire du foyer, la disponibilité alimentaire, le type, la variété et la provenance des aliments consommés, le niveau d'insécurité nutritionnelle, etc.) et une mesure de nature «subjective» (basée sur l'évaluation propre de la personne elle-même).

Cette dernière mesure du bien-être alimentaire subjectif peut elle-même se fonder sur des composantes cognitives (l'évaluation par la personne du niveau de satisfaction sur différents facteurs ayant trait à l'alimentation, sa satisfaction alimentaire) et sur des composantes affectives (portant sur les affects ressentis). La mesure du bien-être alimentaire subjectif peut être également de nature globale, et porter sur l'alimentation en général, ou détaillée, et porter sur différents aspects la composant : type et variété d'aliments et leurs modes de mise à disposition, de préparation, de cuisson et de consommation. 


\section{La place de l'alimentation dans l'évaluation du bien-être}

L'alimentation et les activités liées n'ont pas fait l'objet de mesures distinctes en ce qui concerne l'évaluation de la satisfaction de vie et du bien-être, jusqu'aux années 2000. La dimension alimentaire du bien-être a ainsi longtemps été négligée, malgré son importance dans la vie des individus en termes de temps, de ressources nécessaires (personnelles comme économiques) ou de dimensions symboliques et culturelles. Des applications en termes d'impact de l'alimentation sur la qualité de vie ont été d'abord mises en œuvre dans le domaine de la santé, dans des études cliniques sur le diabète, les maladies cardio-vasculaires ou rénales, puis dans une optique strictement nutritionnelle (Jackson et al., 2005). Ce sont donc les modes d'alimentation en lien avec des pathologies qui ont fait l'objet des premiers travaux, en estimant qu'une «mauvaise» alimentation avait des effets néfastes sur l'individu et son bien-être.

Les premières mesures mises en place avaient une visée essentiellement nutritionnelle, qui combinait des indicateurs objectifs et des évaluations subjectives du bien-être, à la fois de façon générale et sur des domaines spécifiques : économiques, psychologiques, de santé, sans inclure toutefois de questions spécifiques sur l'évaluation de l'alimentation. Ainsi en est-il de la Nutrition Quality of Life Survey (Barr et Schumacher, 2003), de l'EAIAM (échelle de l'accès déterminant de l'insécurité alimentaire des ménages) (Frongillo et Nanama, 2006) centrée sur l'insécurité nutritionnelle, comme du questionnaire Quality of Life Factors (Corle et al., 2001), ou encore du Health related Quality of Life index 4 (Guyatt et al., 1993).

\section{Le bien-être alimentaire subjectif}

Outre d'être complémentaire de la dimension objective du bien-être alimentaire à travers des indicateurs individuels ou collectifs, le recours à une approche de «bien-être alimentaire subjectif» vise à remplir plusieurs objectifs. Il invite à (re)considérer l'alimentation comme un facteur du bien-être individuel, ce que l'économie du bonheur met en évidence (Veenhoven, 1995; Barr et Schumacher, 2003; Kahneman et al., 2004), et il permet d'élargir la vision de l'alimentation focalisée sur les questions biologiques (quantités, qualités) pour ne pas présupposer une «rationalité nutritionnelle» des individus dans leur propre expérience alimentaire : il reconnaît et considère ainsi le caractère multidimensionnel de l'alimentation.

Enfin, en se penchant sur le ressenti des individus par rapport à leur propre vécu alimentaire, la notion de bien-être alimentaire permet d'intégrer le point de vue subjectif et les émotions des mangeurs dans l'analyse de leurs comportements alimentaires et de leurs déterminants. Elle permet de contourner la difficulté et les écueils de la détermination normative (comme dans le cas des mesures de sécurité alimentaire par exemple) puisqu'elle revient à considérer que chaque personne est la seule à même de juger sa situation alimentaire en fonction de ses propres critères et priorités, et elle invite à reconnaître que les opinions des profanes ne sont pas moins dignes d'intérêt que celles d'«experts» à propos de questions qui les concernent directement, comme leur alimentation. 


\section{L'évaluation du bien-être alimentaire subjectif}

Face à la dimension nutritionnelle et objectivante des évaluations du bien-être alimentaire, une évaluation de la satisfaction liée spécifiquement à l'alimentation, sans l'inclure dans d'autres domaines plus généraux, semblait ainsi nécessaire. C'est ce que propose l'outil SWLF : Satisfaction with Food related to Life Scale (Grunert et al., 2007), qui constitue une adaptation de la $S W L S$ appliquée à l'alimentation à travers sept questions générales sur la satisfaction liée à l'alimentation : "food and meals are positive elements in my life»; "when I think of my next meal, I only see problems, obstacles and disappointments»; «I am generally pleased with my food»; "food and meals give me satisfaction in daily life»; «my life in relation to food and meals is close to my ideal»; «I wish my meals were a much more pleasant part of my life»; "with regard to food, the conditions of my life are excellent $»^{25}$. Il devient ainsi possible de mettre en évidence quels sont les facteurs objectifs extérieurs à l'alimentation qui ont une influence sur le niveau de satisfaction alimentaire, et dans quelle mesure ce dernier est corrélé à la satisfaction générale de la vie et à ses domaines liés. Des questions complémentaires peuvent porter sur les raisons des niveaux de satisfaction alimentaire vécue, et les changements concrets dans la situation alimentaire pour améliorer sa satisfaction. Les tests de cohérence entre les mesures de satisfaction alimentaire montrent leur solidité et leur cohérence.

Cette mesure peut souffrir des mêmes critiques que celles apportées à la $S W L S$, celles d'une mesure d'un jugement général et abstrait, l'évaluation de la satisfaction est en effet influencée par la sensibilité à l'état émotionnel de la personne au moment de l'enquête. Pour limiter ce biais, certains travaux suggèrent d'ancrer l'évaluation du subjectif sur du concret en faisant porter les mesures sur le souvenir d'expériences récemment vécues, comme le propose le Day Reconstruction Method (DRM).

À travers l'évaluation affective des activités de la veille en situation, l'utilisation du DRM permet l'extraction de toutes les activités ayant trait à l'alimentation : la production (pêche, agriculture, cueillette, chasse, dons et échanges, achats...), la préparation (nettoyage, stockage, découpage, battage...), la cuisson (préparation culinaire, mode de cuisson...), comme la consommation et ses différentes modalités (seul ou en groupe, au quotidien ou de façon festive). Les épisodes peuvent avoir l'alimentation comme activité «focale» et avec «multi-activités», ou comme activité secondaire. Le but est de mesurer l'impact de trois critères qui déterminent le niveau du bien-être alimentaire perçu : les caractéristiques sociales des individus, le niveau des interactions engagées dans l'activité alimentaire, et la façon dont ils vivent et ressentent leurs activités alimentaires au quotidien (Kahneman et al., 2010), leur «satisfaction alimentaire détaillée».

\footnotetext{
25. «Lalimentation et les repas sont des événements positifs dans ma vie»; «quand je pense à mon prochain repas, je ne vois que problèmes, obstacles et déceptions»; «je suis en général content de mon alimentation»; «l'alimentation et les repas m'apportent de la satisfaction dans la vie quotidienne»; «ma vie liée à l'alimentation et aux repas est proche de mon idéal»; «j'aimerais que mes repas soient une partie plus plaisante de ma vie»; «pour ce qui est de l'alimentation, mes conditions de vie sont excellentes» (traduction des auteurs).
} 


\section{Deux cas de mise en pratique : Rapa (îles Australes) et Mali}

La dimension pratique de l'évaluation du bien-être alimentaire subjectif et de ses ajustements en situation d'enquête est traitée à travers deux terrains de recherche. Ceux-ci ont abordé la question alimentaire de façon différente : «globale», à Rapa ${ }^{26}$, en prenant en compte le système alimentaire dans son ensemble (de la production des ressources alimentaires, leur mise à disposition, leur préparation, jusqu'à leur consommation et ses différentes formes) et en replaçant les activités alimentaires parmi l'ensemble des autres activités quotidiennes; et «détaillée» au Malii ${ }^{27}$, en se penchant sur les activités liées à la consommation alimentaire (approvisionnement/ préparation/consommation alimentaires, partage du thé).

Dans les deux cas, le principe du $D R M$ a été utilisé en faisant décrire toutes les activités de la journée de la veille (Rapa), ou en se focalisant uniquement sur les activités en lien avec la consommation alimentaire (Mali), et en évaluant, pour chaque séquence identifiée, les indicateurs spécifiés dans la méthode originelle pour Rapa (émotions et satisfaction), ou uniquement le niveau de satisfaction vécue et ses déterminants (avec des questions ouvertes complémentaires pour justifier des niveaux déclarés) au Mali.

Pour ce qui concerne l'utilisation de ces instruments, comme pour toute évaluation du bien-être subjectif, sa présentation aux personnes enquêtées doit éviter de faire mention d'une recherche sur le bien-être afin d'éviter de biaiser l'évaluation ellemême. Lévaluation a donc été intégrée à une recherche plus large sur la place de l'alimentation dans les différentes activités quotidiennes (Rapa) ou sur l'insécurité nutritionnelle (Mali), et a fait l'objet d'un retour d'information auprès des populations enquêtées (ou au moins de ses représentants) une fois l'enquête terminée. Dans les deux cas, les questionnaires en français ont été traduits dans les langues vernaculaires, puis rétro-traduits en français pour vérifier la conformité de la traduction avec le sens proposé en français.

Pour le terrain polynésien, le questionnaire du $D R M$ avait été testé auprès d'une trentaine de personnes en Polynésie française, et adapté aux spécificités locales (notamment les questions sur le type de logement, les catégories socioprofessionnelles...), puis à celles de Rapa (pour ce qui touche aux activités quotidiennes). Le questionnaire ayant été élaboré pour être auto-administré, en groupe, par des personnes urbaines actives, il pouvait difficilement être complété de la même façon dans une île de type rural comme Rapa. En effet, il s'est très rapidement avéré que son remplissage était long et fastidieux (jusqu'à deux heures) pour les personnes interrogées, et que parfois, notamment pour les personnes âgées, une traduction en langue rapa était nécessaire.

26. Recherche sur les pratiques et représentations alimentaires des habitants de l'île de Rapa (Australes) menée entre 2009 et 2011 à partir d'un travail de terrain pendant sept mois, basé sur des entretiens individuels et collectifs, des observations sur les prestations alimentaires, et sur une enquête quantitative menée auprès de $70 \%$ de la population adulte de l'île à l'aide du $D R M$ (projet Qualirapa financé par l'Agence nationale de la recherche, référence ANR-09-BLAN-0360-02, centre Edgar Morin, IAAC, Paris).

27. Recherche interdisciplinaire sur la culture alimentaire à l'épreuve de la migration sur les populations rurales, urbaines et immigrées du Maroc et du Mali (projet Alimi financé par l'Agence nationale de la recherche, centre Edgar Morin, IAAC, Paris; UMR Moisa, Montpellier; CERTOP, Toulouse; UMR Nutripass, Montpellier). 
De la même manière, au Mali, le questionnaire a été adapté au contexte. Ainsi, la mesure des affects pour chaque séquence alimentaire a finalement été abandonnée, car cette partie d'enquête s'avérait très longue et pénible, et que, d'un point de vue conceptuel, une mesure commune pour l'ensemble des terrains d'étude (trois échantillons de population étaient concernés) était très controversée. Quelles émotions mesurer? Comment sélectionner celles se rapportant à l'alimentation et s'assurer qu'elles pouvaient avoir un sens (et le même) pour les différentes populations? Comment les traduire? Une mesure de la satisfaction alimentaire vécue pour chaque séquence alimentaire a donc été privilégiée, complétée par des questions ouvertes pour expliquer le niveau de satisfaction déclaré, et pour révéler ce qui aurait permis de «gagner un point» de satisfaction.

Pour compléter les questionnaires à Rapa, nous avons aidé dans bon nombre de cas les répondants à remplir leur questionnaire, assisté par deux personnes maîtrisant parfaitement à la fois les langues française, tahitienne et rapa, après avoir administré à ces dernières personnes le questionnaire et les avoir formées à le remplir. Il apparaît donc nécessaire d'adapter les questionnaires utilisés au type de population interrogé, en le simplifiant notablement dans le cas de populations rurales et/ou à faible niveau d'éducation, quitte à rendre moins aisées les comparaisons internationales. Une autre solution est de passer par des enquêteurs qui interrogent et remplissent directement le questionnaire pour les enquêtés. C'est cette solution qui a été privilégiée au Mali, où l'enquête était réalisée dans des populations avec un fort taux d'analphabétisme.

\section{Apports et limites des outils}

Outre les éléments généraux portant sur l'organisation des activités quotidiennes et leur niveau de bien-être ressenti, les apports du DRM concernent essentiellement le niveau relatif de bien-être ressenti au cours des activités alimentaires, en les différenciant en fonction de leur nature : activités de production alimentaire, d'approvisionnement et d'échange, de préparation préalable et de stockage, ainsi que de préparation culinaire et de consommation. Ainsi, il a été montré pour Rapa (Serra-Mallol, 2018) à la fois le rôle central des prestations sociales alimentaires dans la constitution d'un niveau de bien-être particulièrement élevé, et l'importance des interactions sociales dans le degré de bien-être éprouvé durant ces activités alimentaires. L'approvisionnement de groupe (par les travaux agricoles, la cueillette, la pêche etc.), la préparation collective, et la consommation partagée lors de festins communautaires (bien plus que la consommation quotidienne) figurent parmi les activités les plus appréciées en termes de différentiel positif d'affects (Difmax). Si les repas sont en général pris rapidement en semaine à Rapa (88 mn de temps de repas quotidien sur une semaine, contre $117 \mathrm{mn}$ en France métropolitaine et seulement 52 minutes aux États-Unis), l'activité de manger est considérée comme centrale, «focale», dans $75 \%$ des cas à Rapa, contre $56 \%$ des cas en France et seulement $30 \%$ des cas aux États-Unis, révélant l'importance en soi de l'activité alimentaire à Rapa.

$\mathrm{Au}$ Mali, la variable retenue comme indicateur de la satisfaction alimentaire vécue est la mesure qui porte sur la journée de la veille considérée dans son ensemble, c'està-dire la satisfaction alimentaire vécue globale, plutôt que la satisfaction alimentaire 
détaillée correspondant à la moyenne pondérée par le temps des niveaux de satisfaction alimentaire des épisodes de la veille. Un premier résultat porte sur le fait que plus les personnes se sont déclarées globalement satisfaites de leur alimentation de la veille, plus les évaluations de leurs séquences alimentaires sont élevées. Par ailleurs, l'enquête menée a permis de remettre en cause l'idée que la sécurité alimentaire est l'unique déterminant de la satisfaction alimentaire : l'absence d'insécurité alimentaire n'est ni une garantie, ni un prérequis de la satisfaction alimentaire (Lebrun, 2013).

À Rapa, les incompréhensions, et sans doute bon nombre de non-réponses, portent souvent sur la traduction imparfaite d'un affect du français aux langues tahitienne et rapa. Par exemple, le sentiment de «stress» ne se traduit pas exactement dans ces deux dernières langues. Il faut donc proposer des mots vernaculaires, certes précis, mais qui ne traduisent qu'imparfaitement à eux seuls ces affects en français. Des notions préformées utilisées peuvent être mal comprises ou équivoques, comme la notion de «travail» ou d'«emploi». Ainsi, dans les réponses aux activités quotidiennes, quand il s'agissait de codifier l'activité décrite, les activités «travail de subsistance», «pêche», «artisanat», «cueillette», ou encore le «nourrissage des animaux domestiques» n'étaient pas codées dans la catégorie «travail», mais en tant qu'activités distinctes. De la même façon, à la question «quelle est votre situation professionnelle», plusieurs personnes se sont classées dans la catégorie «sans emploi», alors que leurs journées tout au long de la semaine étaient dédiées à ces différentes activités, conformément à la distinction tahitienne entre travail de la terre et travail salarié. L'autoproduction est valorisée à Rapa, mais tout en maintenant la distinction entre ces deux acceptions : la notion «d'emploi» est ainsi strictement associée à la seconde, la première étant vue comme une "activité», sinon un «loisir» (dont l'équivalent n'existe pas dans les langues tahitienne et rapa). D'un autre côté, ce qui peut être a priori considéré comme un «loisir», la lecture par exemple, peut être perçu différemment au travers des pratiques locales : la lecture n'est pas envisagée comme un loisir par les Rapa, mais comme une nécessité faisant partie du travail de réflexion biblique, et souvent limité à la lecture de la seule Bible. De la même façon, passer du temps à préparer ses ustensiles de pêche n'est pas considéré non plus comme un loisir, mais comme un travail préalable à la pêche proprement dite, elle non plus n'étant pas tenue pour un loisir, mais comme une activité, certes source de plaisirs, mais qui permet de nourrir sa famille et de donner à son entourage en s'inscrivant ainsi dans les réseaux locaux d'échange.

Dans le même ordre d'idées, la formation des enquêteurs pour l'administration du remplissage du questionnaire au Mali a révélé une incompréhension de l'échelle de mesure retenue pour l'évaluation de la satisfaction. Celle-ci, composée de cinq smileys présentés dans un ordre supposé universel, portait à confusion car la lecture culturelle de ces icônes s'est avérée totalement différente de celle envisagée. Ainsi, par exemple, le smiley «le plus heureux» dans notre lecture pouvait apparaître comme «neutre» pour certains, car souriant et les sourcils levés, celui-ci semblait «peu concerné» par la situation. L'échelle de smileys a donc été remplacée par une échelle sous forme de diagramme, plus simple à comprendre d'après les tests.

Une réflexion est également nécessaire sur les types de réponse apportés : le niveau de notation est soumis à l'influence de la culture sur l'évaluation des affects en sous-évaluant ou au contraire surévaluant certains affects en fonction de ce qui 
est localement valorisé. Il est ainsi avéré que certains affects sont normativement «inacceptables» pour certaines cultures (le stress à Rapa), et d'autres «socialement valorisés », tel que le fait d'être heureux à Rapa, ce qui explique le niveau très élevé de satisfaction et de bien-être subjectif atteint à Rapa et en particulier sur les activités alimentaires, vécues non seulement comme vectrices de lien social à travers des activités engageantes, mais comme garantes de la pérennité culturelle autour du «faire ensemble» et de la maîtrise collective des ressources. De même, il a été constaté au Mali des niveaux de satisfaction alimentaire vécue déclarés très élevés, «inattendus» au regard des situations d'insécurité nutritionnelle objectives mesurées par un certain nombre d'indicateurs «classiques»: IMC (indice de masse corporelle), indice de diversité, échelle de l'accès mesurant l'insécurité alimentaire des ménages des personnes enquêtées. Après avoir réalisé un certain nombre de tests pour s'assurer que les résultats étaient fiables (c'est-à-dire que la méthode d'évaluation était bien comprise et rendait compte de réponses qui n'étaient ni infondées, ni aléatoires), nous envisageons que ces résultats soient en partie dus à un «biais culturel », qu'il s'agisse d'un biais d'optimisme, d'un biais de représentation sociale, ou d'une influence des croyances religieuses par exemple.

Du fait de ses présupposés normatifs, un instrument de mesure trop structuré empêche la prise en compte des variabilités et des interprétations locales. Il devrait systématiquement être mis en œuvre dans le cadre d'une recherche et d'un éventail méthodologique plus larges, de type ethnologique, l'incluant et lui donnant sa signification dans son contexte local. Ainsi, les réponses aux questions qualitatives sur les raisons de la satisfaction déclarée et les moyens de l'augmenter pour chaque séquence nous ont permis d'aller plus loin dans la compréhension de ces résultats au Mali, en révélant par des analyses économétriques (régressions logistiques) que les niveaux d'insécurité nutritionnelle objective des individus n'étaient que peu déterminants dans leur satisfaction alimentaire. Au contraire, comme nous l'avions envisagé, toutes les autres dimensions (sociale, hédonique ou identitaire) de l'alimentation peuvent compter dans le jugement que les individus portent sur leur vécu alimentaire, et la hiérarchie de ces dimensions dépend des contextes/environnements des individus comme de leurs préférences personnelles. Il n'y a ainsi pas nécessairement de supériorité de la fonction biologique, même en situation de vulnérabilité nutritionnelle. De la même façon, le terrain ethnologique de sept mois dans le cadre duquel a été soumis le $D R M$ à Rapa a permis, par le biais d'entretiens individuels et de groupe, ainsi que d'observations participantes ${ }^{28}$ quotidiennes, d'éclairer les réponses au $D R M$ et de replacer le fait alimentaire dans son contexte plus général, en fonction des normes sociales et valeurs locales, et du rôle central qu'il revêt dans les activités quotidiennes, les enjeux politiques et les représentations collectives des Rapa.

De ce fait, nous défendons une articulation entre approche quantifiée et approche qualitative du bien-être alimentaire subjectif. Cette articulation nous semble indispensable pour intégrer l'analyse des résultats bruts provenant des outils de mesure du bien-être alimentaire subjectif dans une démarche plus large de type compréhensif. Les données quantitatives recueillies peuvent ainsi être contextualisées et interprétées en tenant compte des normes et valeurs dominantes, et du cadre de

28. C'est-à-dire où l'observateur est impliqué dans la vie et les activités du groupe observé (Serra-Mallol, 2012). 
pensée spécifique de la communauté étudiée. Il s'agit, en somme, d'un exercice d'équilibre épistémologique entre, d'un côté, la meilleure prise en compte possible de la dimension émique (comme définie dans l'introduction de ce manuel) des phénomènes étudiés et, de l'autre, le «calibrage» des outils de quantification du bien-être alimentaire subjectif, qui est la garantie de la comparabilité des résultats obtenus dans des sociétés variées.

Pour conclure ce chapitre, les travaux en sciences sociales sur le bonheur et le bien-être se sont multipliés ces dernières décennies, sans pour autant en poser une définition équivoque ni des indicateurs définitifs, et leur application à l'alimentation est très récente. L'approche par la notion de bien-être alimentaire subjectif paraît la plus fructueuse par sa prise en compte de la perception du bien-être en actes au quotidien et de la dimension autocentrée de l'évaluation.

Au-delà de son intérêt heuristique, la mesure du bien-être alimentaire subjectif permet de rendre apparent le cadre normatif du mangeur dans l'évaluation de son alimentation, les «cadres de l'expérience», et les critères ou déterminants qui la fondent, ainsi que la place et le rôle du fait alimentaire dans les activités quotidiennes et la vie individuelle comme sociale. Complémentaire d'indicateurs «objectifs» traduisant imparfaitement le vécu alimentaire, ces outils remettent le mangeur au centre de l'évaluation, dans une démarche phénoménologique de prise en compte de la façon dont il vit, perçoit et juge lui-même les actes de son quotidien alimentaire.

Deux approches principales ont été présentées : une approche s'inscrivant dans une démarche spécifique de détermination des niveaux de bien-être alimentaire subjectif déclarés et son lien relatif avec le degré d'insécurité alimentaire; et une approche plus globale du bien-être alimentaire subjectif et de ses déterminants, et son importance dans la perception du bien-être général. Dans les deux cas, l'application des outils quantitatifs d'évaluation du bien-être alimentaire subjectif s'est accompagnée d'approches qualitatives et compréhensives pour leur donner du sens, à travers la mise en œuvre de protocoles d'entretiens semi-directifs individuels ou collectifs, sinon de démarches ethnologiques de longue durée sur le terrain.

\section{Références}

Barr J. T., Schumacher G. E., 2003. The need for a nutrition-related quality-of-life measure. Journal of the American Dietetic Association, 103 (2), 177-180. https://doi.org/10.1053/jada.2003.50058

Corle D. K., Sharbaugh C., Mateski D. J., Coyne T., Paskette E. D., Cahill J., Daston C., Lanza E., Schatzkin A., The PTT Study Group, 2001. Self-rated quality of life measures. Effect of change to a low-fat, high-fiber, fruit and vegetable enriched diet. Annals of Behavioural Medicine, 23 (3), 198-207. https://doi.org/10.1207/S15324796ABM2303_7

Diener E., 1984. Subjective well-being. Psychological Bulletin, 95 (3), 542-575. [En ligne] https:// ssrn.com/abstract $=2162125$ (dernière consultation le 25/08/2021).

Diener E., Emmons R. A., Larsen R. J., Griffin S., 1985. The Satisfaction With Life Scale. Journal of Personality Assessment, 49 (1), 71-75. https://doi.org/10.1207/s15327752jpa4901_13

Easterlin R. A., 1974. Does Economic growth improve the human lot? Some empirical evidence, in David P. A., Reder M. W., Nations and Households in Economic Growth: Esays in honor of Moses Abramovitz, New York, Academic Press, 89-125. 
Frongillo E. A., Nanama S., 2006. Development and validation of an experience-based measure of household food insecurity within and across seasons in northern Burkina Faso. Journal of Nutrition, 136 (5), 1409-1419. https://doi.org/10.1093/jn/136.5.1409S

Grunert K. G., Dean M., Raats M. M., Asger N. N., Lumbers M., 2007. Food in Later Life Team. A measure of satisfaction with food-related life. Appetite, 49 (2), 486-493.

https://doi.org/10.1016/j.appet.2007.03.010

Guyatt G. H., Feeny D. H., Patrick D. L., 1993. Measuring Health related to Quality Life. Annals of Internal Medicine, 118 (8), 622-629. https://doi.org/10.7326/0003-4819-118-8-199304150-00009

Jackson J. A., Kinn S., Dalgarno P., 2005. Patient-centred outcomes in dietary research. Journal of Human Nutrition and Dietetics, 18 (2), 83-92. https://doi.org/ 10.1111/j.1365-277X.2004.00581.x

Kahneman D., Krueger A. B., Schkade D. A., Schwarz N., Stone A. A, 2004. A Survey Method for Characterizing Daily Life Experience: The Day Reconstruction Method. Science, 306 (5702), 17761780. https://doi.org/10.1126/science.1103572

Kahneman D., Schkade D. A., Fischler C., Krueger A. B., Krilla A., 2010. The Structure of WellBeing in Two Cities: Life Satisfaction and Experienced Happiness in Colombus, Ohio; and Rennes, France, in Diener E., Kahneman D., Helliwell J., International Differences in Well-Being, Oxford, Oxford University Press, 16-33.

Lebrun M., 2013. L'économie du bonheur face à l'insécurité nutritionnelle : des Maliens ruraux, urbains et migrants évaluent leur situation alimentaire, Thèse de doctorat en économie et gestion, Montpellier, Montpellier SupAgro, 409 p. [En ligne] https:/www.supagro.fr/theses/extranet/13-0013_Lebrun. pdf (dernière consultation le25/08/2021).

Serra-Mallol C., 2012. Observation participante, in Poulain J.-P., Dictionnaire des cultures alimentaires, Paris, PUF, 960-965.

Serra-Mallol C., 2018. Monetary income, public funds, subsistence consumption: the three components of the food supply in French Polynesia. A comparative study of Tahiti and Rapa Iti islands. Review of Agricultural, Food and Environmental Studies, 99 (1), 37-55.

https://doi.org/10.1007/s41130-017-0057-2

Stiglitz J. E., Sen A., Fitoussi J.-P., 2009a. Richesse des nations et bien-être des individus, Paris, Odile Jacob, $326 \mathrm{p}$.

Stiglitz J. E., Sen A., Fitoussi J.-P., 2009b. Vers de nouveaux systèmes de mesure, Paris, Odile Jacob, $432 \mathrm{p}$.

Veenhoven R., 1995. The Cross-National Pattern of Happiness: Test of Predictions Implied in Three Theories of Happiness. Social Indicators Research 34 (1), 33-68. https://doi.org/10.1007/BF01078967 


\section{Partie 2 \\ Suivre les mangeurs et les aliments à la trace}





\title{
Chapitre 6 \\ L'ethnocomptabilité : observer, compter et comprendre ce à quoi tiennent les mangeurs
}

\author{
MARGALIDA MULET PASCUAL ${ }^{a}$ \\ a CEMS, EHESS, Paris, France ; ADEMA, Universitat de les Illes Balears; Odela, Université de Barcelone, \\ Espagne.
}

L'ethnocomptabilité ambitionne de saisir les significations et évaluations que les mangeurs associent à leurs propres pratiques alimentaires. Elle conjugue approche ethnographique (observations, échanges informels, immersion dans le quotidien des enquêtés) et travail d'évaluation à toutes les étapes de l'acte alimentaire (pesée des aliments, réalisation de bilans de consommations). Tout au long de l'étude, les enquêtés sont activement impliqués dans la production des connaissances.

Compter ce qui compte pour les gens : tel est le point de départ de l'enquête d'ethnocomptabilité. Elle s'inscrit dans une démarche d'anthropologie de l'évaluation, définie par une interrogation simple, vue de l'intérieur d'un groupe et de ses proches, individuellement et en commun : qu'est-ce qui compte dans la vie? À quoi tiennent les gens? Et à quoi voit-on qu'ils y tiennent?

Les questions peuvent paraître toutes simples, mais elles mettent en cause les habitudes de pensée les plus enracinées. En effet, pour entrer dans les manières réelles de compter, il ne faut pas confondre la mesure et l'évaluation. Or, dans les sciences économiques et sociales, les deux sont généralement confondues : la mesure par les prix est supposée exprimer directement une sorte de valeur économique objective instituée socialement. Il en résulte une occultation des évaluations subjectives. Tout se passe comme si «les évaluations économiques relev[ai]ent de la mesure monétaire et le reste de la vie sociale demeur[ait] dans une pluralité de valeurs » (Cottereau, 2016). Ce cloisonnement est ce que met en lumière la méthode ethnocomptable par le biais d'une ethnographie des évaluations. En décrivant méthodiquement les façons d'évaluer, cette démarche ethnographique s'écarte d'une définition purement économique de la valeur et s'attache à redéployer sur des bases descriptives ce que peut signifier l'idée de bien-être, prise au sens littéral. Dans cette démarche, le cloisonnement entre faits économiques et faits d'un autre ordre, qui serait celui du social, n'est plus tenable : les faits économiques sont considérés comme une variété de faits sociaux. 
La démarche s'inspire des monographies budgétaires publiées dans Les ouvriers européens (Le Play, 1885) et Les ouvriers des deux mondes (1848-1930), réalisées par un collectif d'enquêteurs nommé Société internationale d'études pratiques d'économie sociale (1857-1885) et animé par Frédéric Le Play.

«Si leurs anciens tableaux de comptes ne sont plus compris aujourd'hui, c'est que leur apparente minutie empirique recouvre un refus absolu de l'économie politique classique, refus qui se traduit par une élaboration alternative avec observation directe des phénomènes et ajout de rubriques originales. Cette élaboration comporte une sorte de comptabilité contextuelle, une mesure scientifique in situ qui n'accepte pas d'éliminer les évaluations en usage, qu'elles donnent lieu ou non à des transactions marchandes. En termes plus politiques de l'époque, leur ambition, reprise ici, est de remplacer "la richesse des nations" par "le bien-être des populations" (Zaccaï-Reyners, 2015)».

Revendiquant cet héritage, le livre d'Alain Cottereau et Mokhtar Mohatar Marzok, Une famille andalouse. Ethnocomptabilité d'une économie invisible, fonde la méthode ethnocomptable. Cette ethnographie porte sur une famille d'origine marocaine vivant en Andalousie (Espagne) et disposant de ce fait d'un champ d'expérience permettant la comparaison entre les deux rives de la Méditerranée : Mohammed, Fatima et leurs quatre enfants âgés de 5 à 13 ans. L'enquête se propose de rendre visible une économie «invisible» : invisible au regard des institutions et statistiques officielles, mais évidemment bien concrète aux yeux des milieux concernés. La formule d'enquête est dite multiple et se pratique à deux. M. Mohatar Marzok était installé dans la famille, avec laquelle il entretient un lien de parenté. Il parle les deux langues utilisées par la famille, rifain et espagnol. Chaque soir, il contactait A. Cottereau pendant deux heures, afin de rédiger avec lui des notes destinées à être publiées dans un journal.

«C'est dans cet exercice de description au jour le jour qu'opère l'intérêt d'une sorte d'observation à deux, même si, pour cette phase-là, un seul est sur place : car le débriefing puis l'écriture confrontent des perceptions différentes des choses. La familiarité amène des compréhensions spontanées que la distance réinterroge et, à l'inverse, la non-familiarité amène des étonnements et des questions sur des choses qui seraient passées inaperçues. Nous procédons ainsi à des premières séries de relevés exhaustifs, emplois du temps remplis pour chaque membre de la famille, à compléter au besoin le lendemain : nutrition, entrées et sorties de biens et de services, scènes de vie dans la famille et au travail» (Cottereau et Mohatar Marzok, 2012).

Après observation et collecte des données, l'ethnocomptabilité passe par un échange de réflexions entre les enquêteurs et les enquêtés, échange qui permet un autre regard.

«L'analyse revient après coup sur les opérations dégagées et ce, avec les intéressés eux-mêmes. Cette étape élargit le propos, sans quitter pour autant son ancrage dans le quotidien de la famille [...]. Il permet des mises en perspectives politiques, culturelles, économiques synchroniquement et diachroniquement plus étendues» (Zaccaï-Reyners, 2015).

Les enquêteurs se sont ainsi rendus ensemble sur place pour présenter une première version de leurs résultats aux membres de la famille et ainsi pouvoir réviser, critiquer et compléter le budget avec ces derniers. 
Le séminaire «Pratiques d'enquête et sens de la réalité sociale» animé par Alain Cottereau et Stéphane Baciocchi à l'École des hautes études en sciences sociales (EHESS) de 2008 à 2021 a prolongé ces réflexions méthodologiques et a permis de lancer d'autres enquêtes d'ethnocomptabilité. De ce séminaire est sorti un numéro spécial intitulé «De quoi vivent les pauvres? » dans la Revue des politiques sociales et familiales (2016), présentant des enquêtes d'ethnocomptabilité menées sur différents terrains : France, Jordanie, Espagne et Cuba.

\section{Appliquer l'ethnocomptabilité au domaine alimentaire}

Contrairement à d'autres disciplines comme la nutrition, l'ethnocomptabilité, à l'instar de l'anthropologie sociale, ne propose pas d'étudier l'alimentation d'un point de vue supposé objectif. C'est plutôt une neutralité axiologique qui est recherchée, afin de restituer la cohérence interne des habitudes alimentaires au sein d'un foyer, considérées comme des «effets de logiques culturelles» (Contreras et Gracia, 2005). Seule la compréhension de cette cohérence, au-delà des contradictions qu'elle abrite, permet de rendre compte de l'entrelacement d'éléments aussi disparates que les problématiques d'approvisionnement et leur résolution, la gestion de l'héritage historique des savoir-faire culinaires, l'évolution des rituels de table, l'invention de succédanés pour survivre à la pénurie, ou encore la débrouille pour établir malgré tout un certain équilibre dans les menus.

Se demander «à quoi tiennent les gens?» permet de saisir les significations et la pertinence que les acteurs attachent aux pratiques et consommations alimentaires. Comme le remarquent Cottereau et Mohatar Marzok dans leur monographie, seule une observation directe, c'est-à-dire en participant comme convive aux repas, permet «l'observation des goûts». Cette démarche rend visible les préférences alimentaires et permet d'analyser les expressions de satisfaction, de comprendre les raisons des appréciations en contexte, d'observer la valeur symbolique donnée à certains aliments.

«Plutôt que de jouer aux devinettes à partir de statistiques de consommation, comme si le fait d'acheter exprimait automatiquement une préférence, l'enquête porte sur les expressions visibles de satisfaction et permet de faire commenter le pourquoi des appréciations» (Cottereau et Mohatar Marzok, 2012, p. 274).

L'approche ethnocomptable, par son étude minutieuse de tout ce qui est bu et mangé, part du même type d'exigence que les enquêtes d'écologie : établir des bilans complets sur des périmètres limités. À partir de ces inventaires écologiques exhaustifs de l'alimentation, toutes les dimensions de l'art de se nourrir peuvent être abordées et décrites de l'intérieur. L'ethnocomptabilité propose dans ce cas un test interne de validité propre aux données d'enquête, par confrontation et analyse de concordance des deux sources produites par l'enquêteur et les enquêtés : un bilan complet des entrées par achats, trocs ou cadeaux; et un bilan complet des consommations d'après l'observation directe des repas sur la même période. Ce travail de mise en cohérence est très instructif et permet de se rendre compte que toute enquête par questionnaire, par remplissage de carnets à domicile ou par entretiens aboutit généralement à des problèmes d'estimation des denrées (sur ou sousestimation), quand bien même les interlocuteurs pensent être sincères et réalistes. 
Chaque phase de confrontation des deux sources, par mise en tableau des données recueillies, puis par discussion des résultats avec les interlocuteurs, aboutit souvent à une importante actualisation des données communiquées spontanément et à un repérage, durant les repas, de disponibilités non identifiées lors de l'enquête sur les seules entrées. Les bilans de consommation sont faits à partir de relevés alimentaires complets (achats et consommations) sur des périodes de 15 jours consécutifs, durées analogues aux minimas que préconisent les nutritionnistes pour évaluer la qualité d'un régime. Le fait de mettre à disposition une balance de précision aux enquêtés leur permet de peser avec exactitude les produits consommés. De surcroît, cet objet suscite leur curiosité, elle les intègre davantage au dispositif d'enquête et symbolise, selon eux, la démarche scientifique. Si ce mode d'enquête s'avère précis, il faut toutefois assumer le fait qu'il peut y avoir des manquements à la pesée. Une telle collecte collaborative, jointe à la prise de notes systématique de l'enquêteur qui sera ensuite révisée par les enquêtés, permet de construire des tableaux nutritionnels. Ces derniers peuvent alors être confrontés à des statistiques globales et constituent ainsi des ressources essentielles. Bien entendu, les données récupérées à ce niveau micro-social ne sont pas directement comparables à celles d'un niveau plus macrosocial. Si l'approche ethnocomptable permet de collecter des observations de grande précision, la pratiquer à grande échelle nécessiterait de nombreux enquêteurs et donc un budget très conséquent.

Accompagner les acteurs durant les achats et l'approvisionnement permet par ailleurs de conduire une anthropologie de l'évaluation : relever les motifs d'achat, les options entre produits et sources d'approvisionnements, l'appréciation des prix au moment des transactions, les arbitrages entre possibilités ouvertes sur le moment, les décisions de composition des menus durant les instants de détermination repérés par l'enquête. Ce type d'observation permet de «dévoiler un paysage limité de possibilités en examinant les transactions effectuées et celles qui auraient été envisageables, de faire apparaître les questions pratiques qui conduisent à certaines orientations dans le monde plutôt qu'à d'autres, compte tenu des possibilités d'action qui s'ouvrent [aux enquêtés]» (Le Méner, 2016). L'approche ethnocomptable de l'alimentation permet d'observer de près - autant dans des économies fragiles que dans des économies plus stables - l'activation et la force des réseaux de solidarité, ainsi que les enjeux et avantages que ces relations mettent en œuvre. Ce sont ces réseaux qui permettent, selon le cas, de déceler les produits manquants ou d'en trouver d'autres à meilleur prix. La solidarité est ainsi concrétisée et comptabilisée comme source d'approvisionnement. La contribution du marché «noir» ou de l'autoproduction alimentaire, ressources généralement invisibles dans les recueils statistiques, est ainsi révélée. Cette approche intime permet de sortir du simple rapprochement entre la «consommation» et les «revenus» et d'inclure des observations et évaluations à toutes les étapes de l'acte alimentaire (production, approvisionnement, cuisine et consommation).

\section{\# Aspects éthiques et déontologiques de la méthode}

L'enquête ethnographique, très exigeante sur le plan de l'observation, peut impliquer des remarques et questions gênantes pour les enquêtés. De ce fait, l'instauration progressive d'une relation de confiance est indispensable au bon déroulement de la 
recherche. Dans le cas de l'approche ethnocomptable, le fait de (re)faire les comptes et les budgets des groupes domestiques étudiés nécessite une exigence supplémentaire. Parler d'argent, montrer de manière exhaustive les stratégies pour «joindre les deux bouts» et assurer malgré tout l'alimentation de la famille est, surtout dans les contextes les moins favorisés, très intrusif. L'obstacle peut toutefois être surmonté du fait que les acteurs étudiés - les familles prises dans leur ensemble - jouent généralement le jeu, c'est-à-dire participent activement à la co-construction de l'enquête. Cette co-construction opère à deux niveaux. D'abord, dans le recueil même des données alimentaires : le fait d'avoir des cahiers d'alimentation sur la table, à portée de toute la famille, et que chacun puisse les lire et les compléter donne un point de transparence importante, car l'information est partagée. Les rôles d'informateur et de preneur de notes deviennent interchangeables. Jour après jour, la relation d'enquête se transforme en travail coopératif : les enquêtés, souvent impatients de découvrir les résultats, en viennent à noter eux-mêmes les aliments consommés dans le cahier, leur prix et leur provenance, leur poids, les entrées de nouveaux produits à la maison, sans oublier ceux reçus en cadeaux. Ensuite, les enquêtés sont invités à lire des articles académiques basés sur des données empiriques, ce qui leur permet de saisir le système de notation et d'information, et ce afin de susciter la discussion et, le cas échéant, d'affiner les résultats. De cette manière, les enquêtés s'approprient aussi de ce qu'il importe de révéler.

L'approche ethnocomptable permet par ailleurs de comparer l'alimentation entre différentes périodes et différents ménages. Tantôt des moments d'intimité avec quelques enquêtés, en tête à tête, s'avèrent propices pour partager des perceptions ou vécus plus personnels, tantôt les réunions de famille et d'amis se prêtent à des discussions collectives, où les questions soulevées peuvent susciter des dynamiques d'échange et des repositionnements (Becker, 2003).

\section{W Une approche transdisciplinaire}

L'ethnocomptabilité est une méthode transdisciplinaire. Elle a été définie comme une enquête de «science sociale» au singulier, car elle a l'ambition de regrouper et de faire dialoguer différentes composantes de cet ensemble disciplinaire : anthropologie, économie, sociologie, philosophie et science politique. Ainsi, l'anthropologie et la sociologie sont essentielles pour le travail de terrain, et notamment l'observation participante; l'économie pour les relevés systématiques de comptes au sein des groupes domestiques; la philosophie pour l'analyse du quotidien; enfin, la science politique permet de ne pas figer dans une signification définitive les significations instables, mais au contraire de faire ressortir les processus qui les agitent.

Nous l'avons vu, l'approche ethnocomptable emprunte concrètement aux techniques d'enquête de l'ethnographie, ce qui inclut l'étude des réseaux sociaux et le recueil de récits de vie. Une attention particulière est accordée aux différents points de vue des acteurs - et leur dynamique - à propos d'une même situation. C'est là l'un des avantages du suivi ethnographique au long cours (Cefaï, 2003). De surcroît, elle évite le recueil arbitraire des faits et rend pertinentes certaines absences. En cela, l'ethnocomptabilité apparaît complémentaire d'autres méthodes et disciplines, telles que l'épidémiologie et les sciences de la nutrition. Car même si les techniques 
de recueil de données statistiques ou nutritionnelles peuvent être bien codifiées et contrôlées, il reste un manque de connaissances à l'échelle micro-sociale que seule peut combler l'observation anthropologique directe. Il s'agit effectivement de saisir les charges émotionnelles et les évaluations morales du fait alimentaire à différents niveaux, qu'il s'agisse de l'acte d'achat, de la cuisine ou de la consommation. C'est pourquoi de plus en plus de nutritionnistes font appel aux sciences sociales afin d'appréhender les comportements alimentaires à une échelle plus fine. C'est justement ce niveau d'analyse micro-social et situé qui peut aider à améliorer et à articuler des méthodes d'analyse macro-sociales et basées sur une raison statistique, comme l'épidémiologie nutritionnelle. Enfin, en participant aux repas familiaux, l'enquêteur peut comprendre et relever les charges émotionnelles et les évaluations morales. Autant d'éléments qui, selon la nutritionniste Monique Romon (2012), «influencent la perception par le sujet de son alimentation, et donc le rapport qu'il en fait».

\section{" Une étude de cas : la débrouille alimentaire à Cuba ${ }^{29}$}

Dans cette recherche, je me suis attachée à décrire les logiques culturelles et politiques qui traversent l'alimentation sous tous ses aspects : de l'approvisionnement à l'acte de manger, dans leurs dimensions à la fois matérielles et idéelles. Linvestigation a été menée au sein des différents ménages de la famille Vázquez, une famille étendue et multi-située, c'est-à-dire établie sur plusieurs sites. La famille Vázquez a été prise à la fois comme base d'observation de la société et comme objet d'enquête. L'ethnographie par immersion a été échelonnée sur sept années (20072013). La principale implantation des ménages est à La Havane (Cuba); une autre est en milieu rural, à Monte, dans l'est de l'île de Cuba, d'où est originaire la famille; une troisième est située aux États-Unis, à Miami. Les membres de ces ménages voyagent ou migrent d'un site à l'autre. S'installer en milieu rural fut, pour moi, une étape importante de la recherche : la connaissance de deux environnements sociaux très différents, mais en contact permanent, a permis de saisir nombre de références internes à l'île. De surcroît, les rencontres ultérieures avec la famille de Monte en déplacement à La Havane ont approfondi les récits de vie et ont permis un suivi ethnographique des trajectoires biographiques. Toutefois, l'enquête multi-site n'a été complète qu'après une période d'observation participante de deux mois au sein de la famille Vázquez à Miami. Là-bas, j'ai été confrontée à des difficultés de nature différente. Dans la vie américaine, une grande partie du temps est absorbée par le

29. Ce travail est tiré d'une thèse de doctorat en anthropologie sociale (Mulet Pascual, 2016). Voici quelques éléments explicatifs de la situation politique et alimentaire de Cuba au moment de l'enquête (2007-2013). Cuba a vécu la sortie de Fidel Castro du pouvoir en 2008. Léconomie de faveur, alors implantée par le Venezuela vis-à-vis de Cuba, permettait une certaine souplesse économique du régime. De surcroît, l'instauration de l'ALBA (Alianza Bolivariana para los Pueblos de Nuestra América), en tant qu'institution commerciale et coopérative entre pays d'Amérique latine, permettait de trouver sur l'île des produits alimentaires de toute sorte et provenant de pays amis, tels que la Bolivie, l'Équateur ou encore le Nicaragua. Des produits en provenance de Chine et d'Iran - autres «amis» du régime - étaient également disponibles. Les relations avec les États-Unis étaient très tendues lorsque George Bush était au pouvoir, puis se sont améliorées avec l'arrivée de Barack Obama (2009), lequel a levé les restrictions de voyage, ainsi que l'envoi d'argent et de courrier postal entre les deux pays. Les relations avec la Russie ont cessé avec la disparition de l'URSS en 1991. 
travail hors domicile et les déplacements, les distances à parcourir étant longues. De ce fait, la maison était souvent vide, à la différence de La Havane ou de Monte, où il y avait toujours des gens à demeure avec qui mener le travail d'enquête. Finalement, j'ai passé plusieurs week-ends sur place et recueilli des données moins détaillées qu'à Cuba, notamment sans pouvoir réaliser de suivi alimentaire.

Pour élargir l'ethnographie des économies domestiques et alimentaires à Cuba, sans toutefois pouvoir pousser aussi loin les inventaires exhaustifs que chez les Vázquez, j’ai analysé deux autres familles aux caractéristiques socio-économiques différentes : les Lopez, deux vieilles femmes à la retraite vivant seules dans un quartier populaire, et les Rodriguez, un couple et sa fille adolescente sans accès à des remesas (mandats) de l'extérieur.

Au début de cette recherche, une question s'imposait : s'agissant de familles en difficulté économique, se nourrissaient-elles bien ou mal au regard des normes nutritionnelles en vigueur? La gêne économique influençait-elle la nutrition? Les personnes étaient-elles satisfaites ou éprouvaient-elles un sentiment de privation? (Cottereau et Mohatar Marzok, 2012, p. 267).

\section{La «cuisine» de la pratique ethnocomptable}

En premier lieu, il s'est agi de trouver comment établir une relation de confiance avec les enquêtés, condition essentielle pour assurer la fiabilité des données. L'immersion s'est avérée être le cadre d'enquête le plus adéquat pour atteindre le vécu quotidien des familles étudiées et celui des Cubains en général. Elle a effectivement permis de confronter les pratiques aux récits; elle s'est déroulée en privé tout en permettant de rester constamment connectée à la vie publique; elle a immédiatement permis de tisser des liens avec les trois générations. Un élément d'apparence anecdotique pour la famille a été particulièrement significatif : j'avais apporté d'Europe une balance mécanique de cuisine pour peser les aliments. C'est à partir de la curiosité suscitée par cette balance que j'ai pu expliquer l'objectif de l'enquête et que j'ai alors demandé leur coopération (à commencer par la pesée des denrées alimentaires). La balance incarnait parfaitement l'idée d'une autoobservation scientifique.

L'enquête a procédé comme suit. Les entrées alimentaires et leurs usages ont été relevés exhaustivement par le biais d'une observation directe et d'entretiens lors des approvisionnements, d'achat et de préparation, puis complétés au besoin par des récits rétrospectifs. Les difficultés d'approvisionnement et d'assortiment des menus sont un domaine où se donne à voir l'art de la débrouille; là, le suivi ethnographique des personnes et des produits a été la méthode la plus efficace que j'ai trouvée. Concrètement, les ethnocomptabilités alimentaires ont été réalisées en procédant à des relevés alimentaires les plus complets possible sur des périodes de 15 jours consécutifs. J'ai relevé le prix des achats selon la quantité supposée du produit acheté (je dis «supposée», car la pesée des aliments à domicile a permis de révéler les écarts de pesée en faveur des vendeurs, une pratique courante sur l'île). Les relevés reflètent donc l'argent dépensé, mais sont susceptibles de surestimer quelque peu les quantités de produits achetés. Néanmoins, ils m’ont permis d'entrer plus avant dans la complexité du problème de l'approvisionnement et dans 
la périodisation des achats. Dans le tableau 6.1 sont présentés les menus quotidiens détaillés de la famille (petits-déjeuners, déjeuners, goûters et dîners). Ces menus ont été par la suite traduits en termes nutritionnels dans les catégories et unités de mesures suivantes : kilo/calories, protéines (en différenciant celles d'origine végétale et animale), glucides, lipides et sucres.

L'analyse détaillée des menus de la famille permet de conclure à une certaine homogénéisation des plats cuisinés, des gammes d'aliments utilisés et des types de cuisson pratiqués par rapport à ce qui était consommé avant l'établissement $\mathrm{du}$ rationnement. L'Enquête nationale de consommation et préférences alimentaires $^{30}$ (Porrata, 2009) indique des traits similaires dans les 14 provinces cubaines, confirmant un resserrement des pratiques alimentaires du pays. À Cuba, l'implantation de politiques alimentaire équitables et les différents épisodes de pénurie ont favorisé l'émergence de ce qu'on pourrait appeler un «mangeur singulier», qui correspond à un rétrécissement des styles alimentaires affectant toutes les dimensions de la vie culturelle.

Sur le plan nutritionnel, les familles observées ont un apport de glucides très élevé et un apport de lipides très faible, ainsi qu'une faible proportion de protéines au regard des recommandations de santé publique. Parmi les glucides figurent le sucre, produit très consommé dans une île historiquement productrice de cette denrée, et les hydrates de carbone, nutriments qui donnent une plus grande sensation de satiété. L'enquête, qui court sur plusieurs années, a aussi permis de comparer l'alimentation entre différentes périodes et ménages. Nous verrons qu'il en résulte une forte variabilité entre ménages proches et même entre périodes de la vie d'un même ménage. De surcroît, l'immersion longue a permis de constater l'existence d'une mémoire des pratiques alimentaires de la population sur plusieurs générations. Ainsi, des souvenirs demeurent : de copieux repas de Noël, la variété des boissons gazeuses disponible durant les années 1950 - et toujours disponibles à Miami sous les mêmes marques -, l'abondance des pêches au sirop ou de la viande en conserve provenant de l'Union soviétique durant les années 1980. Les familles n'ont pas oublié non plus que l'aide alimentaire de l'État avait été plus conséquente dans le passé, avec davantage de produits distribués dans le cadre du rationnement, davantage de variété dans les cantines ouvrières, et ceci à des prix beaucoup plus abordables. Ces souvenirs conditionnent la perception de l'alimentation aujourd'hui et la tragédie des restrictions s'exprime par des récits nostalgiques, mais aussi par des blagues sur l'alimentation, quotidiennes à Cuba. Comme le montre le tableau 6.2, sur le plan quantitatif, les ménages souffrent de pénurie alimentaire, davantage que ce à quoi l'on pourrait s'attendre si l'on se fiait aux statistiques internationales sur l'alimentation, à Cuba ainsi qu'aux rares statistiques officielles à l'échelle nationale.

Sur le plan quantitatif, toutes les familles analysées disposent d'apports énergétiques (kilocalories par personne) à des niveaux moyens correspondant à la moitié, voire au tiers, de ceux affichés par le gouvernement cubain (3288 kcal.). Ces chiffres sont très en dessous des niveaux acceptables recommandés par la FAO. Cela étant, certaines estimations nationales sont produites à partir

30. Encuesta nacional de consumo y preferencias alimentarias. 
Tableau 6.1. Exemples de menus de la famille Vázquez (2012).

\begin{tabular}{|c|c|c|c|}
\hline & Lundi & Mardi & Mercredi \\
\hline $\begin{array}{l}\text { Petit-déjeuner } \\
\text { (entre } 8 \text { h et } 10 \text { h) } \\
\text { (5 personnes) }\end{array}$ & $\begin{array}{l}1 \text { cafetière }(25 \mathrm{~g} \text { café }) \text {, } \\
\text { lait en poudre }(70 \mathrm{~g}), \\
\text { sucre }(30 \mathrm{~g}), \text { biscuits } \\
\text { au beurre }(40 \mathrm{~g}) \text {, } \\
\text { pain }(120 \mathrm{~g}), \text { huile }(5 \mathrm{~g}) \\
\text { et sel }(3 \mathrm{~g}), 1 \text { banane } \\
(275 \mathrm{~g}), \text { papaye }(150 \mathrm{~g}) \text {, } \\
1 \text { orange }(200 \mathrm{~g})\end{array}$ & $\begin{array}{l}1 \text { cafetière }(25 \mathrm{~g} \text { café }) \text {, } \\
\text { lait en poudre }(70 \mathrm{~g}), \\
\text { sucre }(30 \mathrm{~g}), \\
\text { pain }(200 \mathrm{~g}) \text { avec } \\
\text { sauce tomate }(50 \mathrm{~g}), \\
1 \text { banane }(80 \mathrm{~g}), \\
\text { papaye }(75 \mathrm{~g})\end{array}$ & $\begin{array}{l}1 \text { cafetière }(25 \mathrm{~g} \text { café }) \text {, } \\
\text { lait en poudre }(70 \mathrm{~g}), \\
\text { sucre }(30 \mathrm{~g}), \\
\text { pain }(200 \mathrm{~g}), \\
\text { sauce de tomate }(30 \mathrm{~g}) \\
1 \text { banane }(200 \mathrm{~g}), \\
\text { papaye }(75 \mathrm{~g})\end{array}$ \\
\hline $\begin{array}{l}\text { Déjeuner (entre } \\
12 \text { h et } 13 \text { h } 30 \text { ) } \\
\text { (5 personnes) }\end{array}$ & $\begin{array}{l}\text { Ragoût de } \\
\text { quimbombó : } \\
\text { quimbombó }(400 \mathrm{~g}) \text {, } \\
\text { oignon }(55 \mathrm{~g}), 3 \text { gousses } \\
\text { d'ail, poivron vert } \\
(50 \mathrm{~g}) \text {, carotte }(80 \mathrm{~g}) \text {, } \\
\text { citrouille }(175 \mathrm{~g}) \text {, } \\
\text { huile }(3 \mathrm{~g}) \text { et sel }(3 \mathrm{~g}) \text {. } \\
\text { Riz }(400 \mathrm{~g}) \text {, } \\
\text { viande de mouton } \\
(125 \mathrm{~g}), \text { huile }(2 \mathrm{~g}) \text {, } \\
\text { oignon }(30 \mathrm{~g}) \\
\text { Salade }(200 \mathrm{~g}) \text {, } \\
\text { tomate }(90 \mathrm{~g}) \text {, } \\
\text { oignon }(50 \mathrm{~g}) \text {, } \\
\text { huile d'olive }(4 \mathrm{~g}) \\
\text { et sel }(3 \mathrm{~g})\end{array}$ & $\begin{array}{l}\text { Riz }(600 \mathrm{~g}), \text { caguama } \\
\text { (tortue) }(375 \mathrm{~g}), \\
\text { poivron vert }(100 \mathrm{~g}), \\
\text { oignon }(110 \mathrm{~g}), \\
\text { huile soja }(8 \mathrm{~g}), \text { sel }(3 \mathrm{~g}) \\
\text { Bettes bouillies }(400 \mathrm{~g}) \\
\text { Salade }(350 \mathrm{~g}), \\
\text { tomate }(110 \mathrm{~g}), \\
\text { laitue }(105 \mathrm{~g}), \\
\text { betterave }(160 \mathrm{~g}), \\
\text { huile d'olive }(5 \mathrm{~g}) \\
\text { et sel }(3,5 \mathrm{~g})\end{array}$ & $\begin{array}{l}\text { Poivrons farcis : } \\
8 \text { poivrons verts } \\
(800 \mathrm{~g}) \text {, dinde hachée } \\
(230 \mathrm{~g}) \text {, ail }(5,5 \mathrm{~g}), \\
\text { coriandre }(0,60 \mathrm{~g}) \text {, } \\
\text { oignon }(160 \mathrm{~g}), \\
\text { tomate }(150 \mathrm{~g}), \\
\text { huile }(3 \mathrm{~g}) \text { et sel }(3 \mathrm{~g}) \text {, } \\
\text { poivre noir }(0,75 \mathrm{~g}) \text {, } \\
\text { piment doux }(0,5 \mathrm{~g}) \text {, } \\
\text { riz }(600 \mathrm{~g}) \\
\text { Bettes bouillies }(50 \mathrm{~g}) \text {, } \\
\text { huile }(2 \mathrm{~g}), \text { sel }(2 \mathrm{~g}) \\
\text { Salade : tomate } \\
(150 \mathrm{~g}), \text { carotte }(90 \mathrm{~g}), \\
\text { huile olive }(4 \mathrm{~g}) \\
\text { et sel }(2 \mathrm{~g}) \\
2 \text { bananes }(125 \mathrm{~g})\end{array}$ \\
\hline $\begin{array}{c}\text { Goûter (entre } \\
16 \text { h et } 17 \text { h } 30) \\
\text { (4 personnes) }\end{array}$ & $\begin{array}{l}\text { Petits gâteaux }(125 \mathrm{~g}) \text {, } \\
\text { jus de poire }(55 \mathrm{ml}) \\
\text { Thé noir }(250 \mathrm{~g}) \text {, } \\
\text { sucre }(30 \mathrm{~g}), \\
\text { ananas }(135 \mathrm{~g})\end{array}$ & $\begin{array}{l}\text { Gâteau : farine de maïs } \\
(200 \mathrm{~g}), \text { coco }(300 \mathrm{~g}) \text {, } \\
\text { lait concentré } \\
(380 \mathrm{~g}), \text { sucre }(50 \mathrm{~g}), \\
\text { beurre }(20 \mathrm{~g})\end{array}$ & $\begin{array}{l}\text { Biscuits sucrés }(150 \mathrm{~g}) \text {, } \\
\text { tablette de chocolat } \\
(50 \mathrm{~g}) \text {, glace fraise } \\
(100 \mathrm{~g}), 1 \text { cafetière } \\
(25 \mathrm{~g} \text { de café }) \\
\text { sucre }(30 \mathrm{~g})\end{array}$ \\
\hline $\begin{array}{c}\text { Dîner (entre } 20 \text { h } \\
\text { et } 21 \text { h) } \\
\text { (5 personnes) }\end{array}$ & $\begin{array}{l}\text { Salade froide : } \\
\text { pâtes }(200 \mathrm{~g}), 1 \text { boîte } \\
\text { de thon, ananas }(75 \mathrm{~g}) \\
\text { Mayonnaise }: 2 \text { œufs, } \\
\text { huile }(8 \mathrm{~g}), \text { ail }(5 \mathrm{~g}) \text {, } \\
\text { oignon }(50 \mathrm{~g}) \text {, sel }(4 \mathrm{~g}) \\
3 \text { paquets de biscuits } \\
(60 \mathrm{~g}) \\
\text { Pain }(100 \mathrm{~g}) \\
\text { Riz et quimbombó } \\
\text { de midi }+ \text { pommes } \\
\text { de terre }(300 \mathrm{~g})\end{array}$ & $\begin{array}{l}\text { Soupe : poulet }(125 \mathrm{~g}) \text {, } \\
\text { malangas }(500 \mathrm{~g}), \\
\text { citrouille }(100 \mathrm{~g}), \\
1 \text { banane verte }(260 \mathrm{~g}) \text {, } \\
\text { bette }(100 \mathrm{~g}) \text {, ail }(5 \mathrm{~g}) \text {, } \\
\text { oignon }(70 \mathrm{~g}), \\
\text { coriandre }(0,2 \mathrm{~g}) \text {, } \\
\text { sel }(4 \mathrm{~g}), \text { huile }(4 \mathrm{~g}) \text {, } \\
\text { yucca bouilli }(100 \mathrm{~g})\end{array}$ & $\begin{array}{l}1 \text { paquet } \\
\text { de spaghetti }(400 \mathrm{~g}) \text {, } \\
\text { sauce tomate }(220 \mathrm{~g}) \text {, } \\
\text { fromage râpé }(100 \mathrm{~g}) \text {, } \\
\text { origan }(1 \mathrm{~g}) \\
\text { Soupe : viande } \\
\text { de mouton }(450 \mathrm{~g}) \text {, } \\
\text { malanga }(475 \mathrm{~g}) \text {, } \\
\text { citrouille }(75 \mathrm{~g}) \text {, banane } \\
\text { verte }(275 \mathrm{~g}), 4 \text { gousses } \\
\text { d'ail, vermicelles }(30 \mathrm{~g}) \text {, } \\
\text { huile }(3 \mathrm{~g}) \text {, sel }(3 \mathrm{~g}) \\
\text { Salade : tomate }(150 \mathrm{~g}) \text {, } \\
\text { bette }(150 \mathrm{~g}) \text {, } \\
\text { huile }(3 \mathrm{~g}) \text {, } \\
\text { sel }(2 \mathrm{~g})\end{array}$ \\
\hline
\end{tabular}


d'extrapolations économiques, comme c'est le cas dans les trois études portant sur la consommation alimentaire et réalisées par l'ONE (Bureau national des statistiques) du gouvernement cubain en 2004, 2005 et 2006. Dans ces études, ni le contexte ni la méthodologie utilisée pour le recueil des données ne sont définis, ce qui suscite des doutes quant à leur fiabilité.

Tableau 6.2. Consommations moyennes quotidiennes (en kilocalories par personne). Comparaison de nos données avec les moyennes statistiques nationales publiées par l'État cubain et la Food and Agriculture Organization (FAO).

\begin{tabular}{ccccc}
\hline Année & \multicolumn{1}{c}{$\begin{array}{c}\text { Familles } \\
\text { enquêtées }\end{array}$} & $\begin{array}{c}\text { Moyennes } \\
\text { des enquêtes directes }\end{array}$ & $\begin{array}{c}\text { Données } \\
\text { de l'État cubain }\end{array}$ & $\begin{array}{c}\text { Donnés } \\
\text { de la FAO } \mathbf{~}^{\mathbf{2}}\end{array}$ \\
\hline $\mathbf{2 0 0 3}$ & - & - & 2936,0 & 3246,0 \\
\hline $\mathbf{2 0 0 4}$ & - & - & 3226,5 & 3346,0 \\
\hline $\mathbf{2 0 0 5}$ & - & - & 3245,5 & 3254,0 \\
\hline $\mathbf{2 0 0 6}$ & - & 1344,01 & 3268,4 & 3260,0 \\
\hline $\mathbf{2 0 0 7}$ & $\begin{array}{l}\text { Famille Vázquez } \\
\text { (La Havane) }\end{array}$ & 1244,23 & $3288^{3}$ & 3251,0 \\
\hline $\mathbf{2 0 1 0}$ & $\begin{array}{l}\text { Famille Vázquez } \\
\text { (La Havane) }\end{array}$ & 1589,94 & - & 3159,0 \\
& $\begin{array}{l}\text { Famille López } \\
\text { Famille Vázquez } \\
\text { (Monte) }\end{array}$ & 1195,47 & & 3277,0 \\
\hline $\mathbf{2 0 1 2}$ & $\begin{array}{l}\text { Famille Vázquez } \\
\text { (La Havane) }\end{array}$ & 1431,53 & & \\
& $\begin{array}{l}\text { Famille López } \\
\text { Famille Rodríguez }\end{array}$ & 1451,02 & & \\
\hline
\end{tabular}

${ }^{1}$ ONE (Oficina Nacional de Estadística) de Cuba, 2013, non publié. Précisons que les données les plus récentes fournies par Cuba datent de la période 2003-2006, ce qui rend la comparaison impossible avec les nôtres (recueillies en 2007, 2010, puis 2012). Nous pouvons toutefois envisager l'important décalage entre les deux sources.

${ }^{2}$ Disponibilités alimentaires selon la FAO : http://www.fao.org/faostat/en/\#data (dernière consultation le $08 / 09 / 2021$ ).

${ }^{3}$ Chiffres officiels cités dans Ramos Lauzurique, 2010.

\section{Adaptation de la méthode au contexte cubain}

À Cuba, pour s'approvisionner, il faut toujours anticiper et être vigilant. L'approvisionnement est chronophage, il entraîne des heures de queue et oblige à s'insérer en permanence dans des systèmes d'entraide et de faveurs. C'est un cercle vicieux : l'insertion nécessaire dans ces réseaux augmente l'anxiété quotidienne. De ce fait, la méthode d'enquête s'est adaptée à la réalité cubaine en établissant des distinctions selon les différents circuits d'approvisionnement, ce qui a certes complexifié encore les tableaux de relevés, mais cela a permis de saisir ces circuits et leurs places respectives du point de vue des utilisateurs. Le tableau 6.3 récapitule les circuits empruntés, la fréquence et la simultanéité des visites selon les jours de la semaine, ainsi que les dépenses faites à chaque fois. 


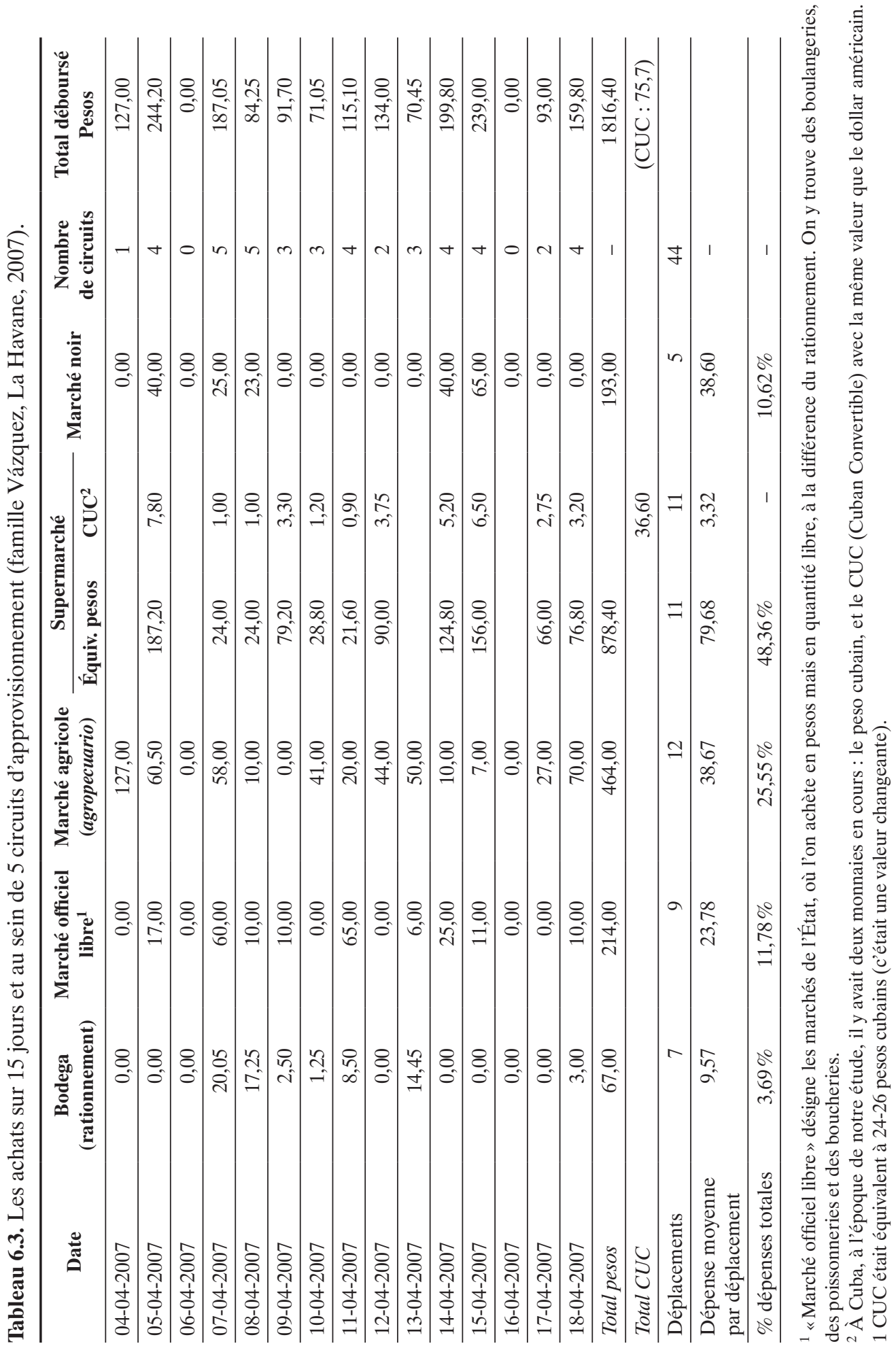




\section{Encadré 6.1 : Évaluations des enquêtés à propos de l'alimentation à Cuba}

\section{Les achats expriment la liberté des choix}

Juana, grand-mère de la famille Vázquez (6 octobre 2010, La Havane) :

«Ici, rien n'est sûr et du fait qu'il y a peu de protéines, il faut s'assurer au minimum des œufs. Aujourd'hui, on m'a proposé des œufs au marché noir après plus d'un mois sans en avoir mangé et je les ai achetés même s'ils étaient chers par peur d'en manquer. Peut-être que demain, il n'y en aura plus au marché noir, et à la bodega ce n'est pas sûr. Et alors, qu'est-ce que je fais?»

\section{Le rapport qualité-prix est le plus important quand on achète}

Juana, en regardant un sac avec des morceaux de poulet surgelé (15 octobre 2010, La Havane) :

«C'est cher, mais c'est mieux que d'autres produits car ceux-ci sont sans graisse et pas comme le poulet du rationnement, où il n'y a que la peau. Ainsi, ce sac sert pour deux repas complets de toute la famille.»

On remarque ici que la perception que Juana a d'un produit bon marché passe par son exigence que le produit «rinda» («rende», ou «soit rentable») c'est-àdire qu'il serve pour plusieurs repas.

\section{On paie selon la quantité que l'on achète}

Journal de terrain (26 janvier 2012, La Havane) : Juana est allée acheter des petits poivrons au marché agricole. La mesure est une boîte de conserve de $500 \mathrm{~g}$ remplie de poivrons, à 6 pesos. Quand la vendeuse est allée peser, Juana s'est rendu compte que dans la boîte il y en avait une autre plus petite. De cette manière, elle vendait une moindre quantité de produit pour le même prix.

Le rationnement alimentaire correspond à une quantité fixe distribuée par mois Journal de terrain (avril 2007, La Havane) : lorsque nous avons pesé les 5 livres de sucre que Juana avait rapportées de la bodega, nous avons vu qu'il en manquait un peu plus d'une livre : il n'y en avait que 3,93 livres. De plus, l'arrivée des produits sur les points de distribution est aléatoire et instable, ce qui ne permet pas une planification alimentaire.

Journal de terrain (29 septembre 2010, La Havane) : Juana est allée chercher le poulet du mois, c'est-à-dire que l'avant-dernier jour du mois, le poulet du rationnement n'était pas encore parvenu aux familles.

\section{Le rationnement apporte le panier de base à toutes les familles}

Journal de terrain (2007, La Havane) : le riz du mois d'avril était terminé le 13 du mois. Chaque jour, les Vázquez consomment environ $800 \mathrm{~g}$ de riz, l'aliment de base. Juana doit acheter chaque mois dix livres de riz au marché noir.

Les frijoles (haricots secs) : le 19 avril, le quota était fini. La famille a dû acheter pour finir le mois entre dix et quinze livres de frijoles au marché libre (non rationné) à huit pesos la livre.

Le quota d'huile qu'ils reçoivent est de $25 \mathrm{cl}$ par personne soit 1,25 1 par mois pour les cinq. Cette quantité est consommée en huit jours. 
La somme dépensée chaque mois à la bodega est de loin la dépense la plus faible de la famille Vázquez. Cette situation est due aux bas prix des produits sur ce marché, subventionnés et rationnés par l'État. L’importance des dépenses sur les autres marchés reflète en même temps l'extrême insuffisance de l'approvisionnement dans le système de rationnement et la nécessité de se fournir en dehors de la canasta básica (panier de base) du plan.

Les chiffres du gouvernement cubain sur l'importance des différentes sources de distribution dans l'approvisionnement des familles divergent grandement par rapport à mes observations. Certes, les données officielles tiennent compte de l'alimentation publique (restaurants d'État, cantines ouvrières) et de la consommation dite sociale (dans des centres d'éducation, de santé publique et dans les centres sportifs). Dans mes budgets, je ne leur ai pas consacré de compte à part, car ni la cantine ouvrière ni l'alimentation pendant un séjour à l'hôpital n'ont réduit les achats de nourriture du budget familial, car, même en prenant leur déjeuner sur le lieu de travail, les membres de la famille venaient très souvent manger à la maison en raison de la mauvaise qualité de la nourriture proposée. De même, presque aucun des repas proposés à l'hôpital n'a été consommé en entier en raison de la préférence donnée aux repas préparés à la maison. En revanche, les données officielles ne tiennent pas compte d'un marché très important dans le quotidien des familles cubaines : le marché noir (mercado negro), invisible dans les rapports et les statistiques. Néanmoins, les données officielles font une place à l' «autoconsommation» estimée (autoconsumo), ce qui a un sens à la campagne, mais n'en a guère en ville; dans le cas des observations à La Havane, l'autoconsommation au sens statistique officiel est nulle. La méthode ethnocomptable a ainsi permis de prendre de la distance avec certaines données et discours du gouvernement cubain (tout comme avec certains des postulats de la théorie économique néoclassique). L'encadré 6.1 présente quelques évidences remises en question.

Le but de cette enquête n'était pas de poser de façon définitive des significations et valeurs associées au fait alimentaire. Au contraire, il était de montrer, dans un contexte donné, leur instabilité et le rôle des facteurs émotionnels et moraux dans leur recomposition. L'ethnocomptabilité permet de saisir finement ces processus de redéfinition et peut alors être utile pour la mise en place de politiques publiques (alimentaires, de santé publique...), ainsi que pour des projets de développement ou d'action sociale visant à améliorer le bien-être des citoyens.

\section{W Références}

Becker H., 2003. Inférence et preuve en observation participante. Fiabilité des données et validité des hypothèses, in Cefaï D. (éd), L'enquête de terrain, Paris, La Découverte, MAUSS, 624 p. (coll. Recherches/MAUSS).

Contreras J., Gracia M., 2005. Alimentación y cultura. Perspectivas antropológicas, Barcelona, Editorial Arial, 505 p.

Céfaï D., 2003. L'enquête de terrain, Paris, La Découverte, 624 p.

Cottereau A., Mohatar Marzok M., 2012. Une famille andalouse. Ethnocomptabilité d'une économie invisible, Paris, éditions Bouchene, 354 p. 
Cottereau A., 2016. Ne pas confondre la mesure et l'évaluation : aspects de l'ethnocomptabilité. Dossier : Joindre les deux bouts. Enquêtes d'ethnocomptabilité. Revue des politiques sociales et familiales, 123, 11-26. https://doi.org/10.3406/caf.2017.3179

Le Méner E., 2016. Introduction. Dossier : Joindre les deux bouts. Enquêtes d'ethnocomptabilité. Revue des politiques sociales et familiales, 123, 3-5. [En ligne] https://www.persee.fr/doc/caf_24314501_2017_num_123_1_3176 (dernière consultation, 01/09/2021).

Le Play F., 1885. Les ouvriers européens : étude sur les travaux, la vie domestique et la condition morale des populations ouvrières de l'Europe, et leurs relations avec les autres classes, précédé d'un exposé de la méthode d'observation, Paris, Imprimerie Impériale, 6 vol.

Mulet Pascual M. 2016. "Resolver» : un art cubain de la débrouille. La gestion du quotidien des Vázquez, une famille transnationale dans la Cuba des années 2000, Thèse de doctorat en anthropologie sociale et ethnologie, Paris, EHESS, 613 p.

Porrata C., 2009. Consumo y preferencias alimentarias de la población cubana con 15 y más años de edad. Revista Cubana de Alimentación y Nutrición, 19 (1), 87-105. [En ligne] http://www.revicubalimentanut.sld.cu/Vol_19_1/RCAN_Vol_19_1_Pages_87_105.htm (dernière consultation, 01/09/2021).

Ramos Lauzurique A., 2010. El dilema de la libreta de racionamiento. Revista Hispano Cubana, 37, 75-79.

Romon M., 2012. Enquêtes nutritionnelles, in Poulain J.-P. (éd.), Dictionnaire des cultures alimentaires, Paris, Presses universitaires de France, 470-475.

Société internationale d'études pratiques d'économie sociale, 1857-1885. Les ouvriers des deux mondes, Paris, réed. partielle 1983, Paris, à l'enseigne de l'arbre verdoyant.

Zaccaï-Reyners N., 2015. Pour une science sociale de l'évaluation. Une famille andalouse. Ethnocomptabilité d'une économie invisible. SociologieS. https://doi.org/10.4000/sociologies.4897

\section{Remerciements}

J'aimerais remercier Alain Cottereau pour sa relecture et ses suggestions, ainsi qu'Olivier Lepiller et Tristan Fournier pour le suivi éditorial. 


\title{
Chapitre 7 \\ Photovoice : une méthode participative pour identifier les environnements alimentaires du point de vue des habitants
}

\author{
CAROLYN I. Auma ${ }^{a}$, Michelle HoldSWORTH, \\ ET REBECCA PRADEILLES
}

\footnotetext{
a School of Food Science and Nutrition, University of Leeds, Leeds, United Kingdom.

b UMR MoISA, université Montpellier, CIHEAM-IAMM, CIRAD, INRAE, Institut Agro, IRD, Montpellier, France.

${ }^{c}$ School of Sport, Exercise and Health Sciences, Loughborough University, Loughborough, United Kingdom.
}

La méthode Photovoice mobilise un groupe de participants pour réaliser, commenter, puis exposer des photographies illustrant un thème ou un problème vécu par ce groupe. Cette démarche participative et orientée vers l'action permet l'expression de points de vue propres au groupe et fournit matière à des actions de plaidoyer (telle que l'exposition photographique).

Photovoice, anciennement nommée «photo novella», est une méthode de recherche qualitative, s'appuyant sur la participation d'un collectif ou d'une communauté (Community Based Participatory Research, CBPR), et s'inscrivant dans un projet de changement social à plus ou moins long terme. Le terme Photovoice a été utilisé pour la première fois au début des années 1990. La méthode consiste à demander à un groupe de participants de réaliser des photographies pour présenter leur propre communauté et les changements qu'ils souhaiteraient y apporter (Wang et Burris, 1994).

À ses débuts, Photovoice a été mobilisée par divers courants (féminisme, réalisme, autonomisation par l'éducation, photographie documentaire) avec trois objectifs principaux (Wang et Burris, 1994; 1997) : permettre à des individus de porter un regard réflexif sur leur propre communauté en leur permettant de réaliser un reportage photographique sur celle-ci; susciter un dialogue critique au sein de cette communauté et un diagnostic de ses forces et aspirations, en commençant un débat autour des photographies réalisées; et enfin créer une dynamique collective et déclencher une réflexion spécifique au sein des dirigeants communautaires et des décideurs politiques autour d'objectifs de changement social, au moyen d'exposition de ces photographies (Wang et Burris, 1997; Wang, 1999). 
À l'origine, Photovoice est une méthode de photo-élicitation (c'est-à-dire une technique utilisant des images pour susciter des récits); elle donne la possibilité à des groupes d'individus de décrire leur réalité, d'exprimer la façon dont ils vivent les problèmes parfois complexes auxquels ils ont à faire face (Catalani et Minkler, 2010; Nykiforuk et al., 2011). C'est une forme de documentaire photographique, dont l'une des premières applications a porté sur l'accès aux ressources et les problèmes de santé des femmes de la province rurale du Yunnan en Chine (Wang et Burris, 1997).

\section{W Les étapes de la méthode Photovoice}

Wang a décrit pour la première fois le «protocole Photovoice» en neuf étapes (Wang, 1999). La première étape consiste à identifier la communauté cible au sein de laquelle va être appliquée la méthode; cette étape est généralement réalisée par un membre de l'équipe de recherche.

Ensuite, avec l'aide des leaders de la communauté identifiée, des participants sont sélectionnés, éventuellement sur des critères spécifiques liés à la thématique de recherche, tels que l'âge ou le sexe (étape 2).

Puis, une première réunion de présentation du projet est organisée avec les participants : les objectifs généraux de la recherche et de Photovoice leur sont exposés et une formation de base à la photographie leur est dispensée (lorsque les participants ne possèdent pas déjà ces compétences) (étape 3).

Les participants et les chercheurs discutent également des questions d'éthique liées à l'usage de photographies et du nécessaire «consentement éclairé pour les personnes et leur environnement immédiat représentés dans les photographies » (étape 4).

Après discussion de l'objectif général de la recherche, une question plus spécifique est arrêtée, que les participants devront illustrer par des photographies (étape 5).

Puis, les participants réalisent chacun leur reportage sur une période déterminée (de quelques jours à plusieurs semaines) (étape 6).

À l'étape suivante (étape 7), les participants sont à nouveau réunis pour présenter leurs reportages et discuter des messages que chacun a souhaité illustrer par ses photographies. L'animation des discussions est conduite à l'aide de deux méthodes couramment utilisées : la méthode SHOWeD : que voyez-vous (See) ici? Que se passe-t-il sur cette photographie (Happening)? En quoi cela se rapporte-t-il à nos (Our) vies? Pourquoi (Why) cette situation (problèmes, forces et faiblesses) ? Que pouvons-nous faire $(D o)$ ?; ou la méthode PHOTO : pourriez-vous parler ou décrire votre photo (Photo)? Que se passe-t-il (Happening) sur votre photo? Pourquoi avezvous pris une photo de $(O f)$ ceci?; Que nous dit (Tell) cette photographie et en quoi illustre-elle des possibilités (Opportunities) de changements?

Ensuite, les données collectées (c'est-à-dire les photographies avec leurs légendes mettant en évidence ce que les participants ont voulu montrer et les récits qu'elles ont permis de faire émerger au sein du groupe de participants-reporters) sont partagées avec les autres membres de la communauté, et les dirigeants communautaires locaux ou les décideurs politiques à l'occasion d'une exposition (étape 8). 
Photovoice n'est pas seulement une «nouvelle méthode attrayante» de collecte de données qualitatives. Photovoice est une autre façon de faire de la recherche en donnant, comme son nom l'indique, «plus de voix aux sans-voix». L'objectif est de donner aux participants la possibilité de s'exprimer à travers leurs photographies et leur exposition à un public plus large. C'est de cette possibilité nouvelle d'expression qu'est attendu un changement social (étape 9).

Dans ce chapitre, nous nous concentrons essentiellement sur les étapes 1 à 8 .

Photovoice est une méthode participative $(C B P R)$, dans laquelle les niveaux de participation peuvent varier. Dans les premières applications, les étapes initiales (définition des thèmes et des objectifs de la recherche) étaient conduites par l'équipe de recherche, les membres de la communauté n'étant mobilisés que dans les étapes suivantes pour la réalisation des reportages, et leur mise en discussion. Par la suite, la participation des membres de la communauté s'est progressivement étendue (Johnston, 2016). Actuellement, divers niveaux de participation peuvent être observés; depuis un engagement des participants limité à la réalisation des reportages et à leur discussion, jusqu'à un niveau d'engagement élevé incluant une implication dans la conception de la recherche comme dans la diffusion et l'utilisation des résultats.

Une participation élevée est plus probable dans les communautés où une relation est déjà établie entre les chercheurs et la communauté (Johnston, 2016) et lorsque les effectifs de participants sont relativement faibles. Bien que l'objectif soit généralement d'obtenir des niveaux élevés de participation, un équilibre est à trouver entre les contraintes de ressources - coût et temps - et les avantages potentiels que les participants et les communautés pourraient tirer d'une participation accrue au projet (Wang et Burris, 1997; Johnston, 2016).

\section{Avantages et limites de la méthode Photovoice}

Photovoice est un outil très adaptable, qui peut être donc être utilisé pour étudier un large éventail de sujets (Wang et Burris, 1997), incluant notamment la santé et l'alimentation. De plus, Photovoice est une méthode participative, qui place les parties prenantes (les participants) au centre de la démarche de recherche et leur permet de contrôler les processus et de s'en approprier les résultats (Wang et Burris, 1997; Johnston, 2016).

La méthode s'inscrit dans une démarche de recherche-action (voir chapitre 13) reposant sur une approche globale et approfondie des processus étudiés. En confiant des appareils photographiques aux participants, c'est leur «point de vue» sur leur communauté et le «cadrage» de leurs propres problèmes qui sont privilégiés, plus précisément que ne pourraient en rendre compte des personnes extérieures à ces communautés (Wang et Burris, 1994; 1997; Wang, 1999).

Ceci est particulièrement important lorsque l'on travaille avec des groupes d'individus dont le statut social est peu ou moins reconnu, tels que les femmes, les habitants des zones rurales, les groupes défavorisés, ou les personnes socialement stigmatisées, notamment pour des raisons de santé. De plus, l'utilisation d'un appareil 
photographique peut être mise à la portée de tous. Photovoice peut s'adresser à des personnes non alphabétisées (Wang et Burris, 1994), comme cela est souvent le cas dans les zones rurales de nombreux pays à revenus faibles et intermédiaires.

Photovoice présente des atouts supplémentaires par rapport aux autres méthodes d'enquête qualitatives. Tout d'abord, l'opportunité qui est offerte aux participants d'apprendre à utiliser un appareil photographique et de réaliser un reportage peut constituer un attrait et une motivation supplémentaires, notamment dans le cas de photographes novices (Wang et Burris, 1994). Photovoice permet aux participants d'exprimer un ressenti par la photographie qu'ils ne pourraient pas verbaliser par des méthodes plus classiques. En outre, les photographies réalisées vont permettre aux participants de garder une trace durable de leur collaboration avec les chercheurs, et leur fournissent un outil de plaidoyer qu'ils pourront mobiliser de façon autonome dans d'éventuelles luttes sociales ultérieures (Wang et Burris, 1994; 1997; Johnston, 2016).

Dans le domaine spécifique de l'alimentation, la méthode, en plaçant les participants au centre du processus de recherche, apporte une compréhension approfondie des comportements alimentaires et de leurs déterminants du point de vue des participants eux-mêmes (c'est donc une démarche émique plutôt qu'étique). Les photographies réalisées peuvent rendre compte, par exemple, de la représentation que les participants ont de leurs propres régimes et pratiques alimentaires, des différents aliments qu'ils consomment, de la façon de les combiner et de les préparer, offrant ainsi un support, par la discussion de ces photographies, à une exploration approfondie des facteurs à la base de ces comportements. Par exemple, lors d'une étude en Ouganda, de façon tout à fait inattendue pour les chercheurs, une participante a choisi d'illustrer les contraintes matérielles qui influencent les comportements alimentaires de sa communauté par la photographie d'une route. Par cette photographie, elle souhaitait illustrer le rôle de la relation qu'elle entretenait avec le vendeur chez qui elle s'approvisionnait régulièrement en produits frais. Cette photographie, dans un raccourci saisissant, illustre les motivations réelles qui ont conduit la participante à choisir ce lieu d'approvisionnement (accessibilité et proximité).

Même si cette méthode présente de très nombreux avantages dans le cadre de recherches qualitatives visant une approche globale et approfondie, elle présente également, comme toute méthode, ses propres limites.

Photovoice est une méthode qualitative, par conséquent elle ne peut répondre à des questions de recherche visant à établir des corrélations entre comportements alimentaires, nutrition et santé.

La méthode est potentiellement porteuse de biais. Par exemple, les reportages réalisés par les participants ne visent pas nécessairement à reproduire comment ils voient leur communauté ou leur place dans la communauté, mais peuvent servir à diffuser l'image qu'ils aimeraient en donner. Cela peut être particulièrement le cas lorsqu'il s'agit de traiter de sujets sensibles et éventuellement stigmatisants. De plus, le besoin pour les participants d'obtenir le consentement des personnes photographiées peut les conduire à limiter les sujets de leurs photographies.

Les photographies prises peuvent s'inscrire dans une démarche personnelle du participant, qui peut les utiliser pour présenter sa place dans la communauté, son 
rapport aux normes sociales de son groupe, éventuellement son souhait de s'en démarquer, ou de se présenter proche du groupe auquel il souhaiterait appartenir. Il est important de percevoir ces effets liés aux poids des normes et aux rôles des interactions sociales.

Les participants peuvent se sentir obligés de prendre des photographies, ou peuvent penser qu'ils le «doivent» aux chercheurs, puisqu'ils se sont engagés dans le projet. De plus, si les photographies peuvent être faciles à collecter, en plus des données de discussion de groupe, leur ensemble présente un vaste corpus de données, qui peut être difficile à analyser (Wang et Burris, 1994).

Enfin, il est important de considérer les implications éthiques et déontologiques spécifiques à la démarche Photovoice et qui sont détaillées dans la section suivante (voir «Aspects éthiques, risques et règles de sécurité de la méthode Photovoice»).

\section{Aspects éthiques, risques et règles de sécurité de la méthode Photovoice}

Dès le début du processus, la question de la propriété des photographies prises se pose : appartiennent-elles aux participants ou aux chercheurs? Sur le plan éthique, Photovoice postule que les photographies appartiennent à chacun des participants parce qu'elles sont le produit de sa créativité et constituent la parole que le participant souhaite partager avec le chercheur (Wang et Redwood-Jones, 2001). Afin qu'il puisse utiliser les photographies pour sa recherche, le chercheur doit donc obtenir l'accord écrit des participants via un «formulaire d'acceptation et de décharge» détaillant les activités de recherche pour lesquelles ces photographies vont être utilisées, autorisant leur utilisation à des fins de recherche, et reconnaissant la propriété de l'auteur de la photographie. Le non-respect de cette consigne constitue une violation du droit d'auteur et un vol de propriété.

Photovoice est aussi une méthode éventuellement intrusive, car les participants sont invités à photographier la réalité de leur vie quotidienne et de leur communauté. Lorsqu'il s'agit d'un sujet sensible, ils peuvent se sentir gênés d'exposer ainsi leur situation, et peuvent voir ces photographies comme un douloureux rappel de leur réalité.

Les participants peuvent aussi douter des véritables intentions poursuivies par les chercheurs à travers ces photographies et sont susceptibles, pour cette raison, de refuser de participer au projet Photovoice ou limiter les photographies que le chercheur peut utiliser.

Par ailleurs, les participants ne doivent pas prendre des photographies sur lesquelles des personnes /ou leur environnement matériel sont identifiables sans leur autorisation expresse. Une session de formation est donc organisée en début du projet, au cours de laquelle les participants sont sensibilisés aux questions éthiques liées à l'usage de photographies et à l'importance d'obtenir le consentement écrit des personnes impliquées (Wang et Redwood-Jones, 2001). Des exemplaires d'un formulaire de consentement leur sont distribués. Ce formulaire présente le photographe et le projet de recherche auquel il participe, ainsi 
que les utilisations envisagées pour les photographies prises. Sans l'obtention du consentement des personnes et des propriétaires des biens apparaissant sur les photographies, celles-ci ne pourront pas être utilisées dans le cadre du projet (Wang et Redwood-Jones, 2001).

En outre, les participants s'exposent à quelques risques. Dans certains endroits, les appareils photos qui leur sont confiés peuvent attirer sur eux des tentatives de vols avec éventuellement agressions physiques. Ils peuvent aussi être tentés de se mettre en danger dans le seul but d'obtenir une «bonne photographie». Ces risques doivent être abordés avec les participants avant qu'ils ne démarrent leur reportage. Enfin, lorsque Photovoice s'inscrit explicitement dans un objectif interventionnel, le risque est que le changement obtenu ne corresponde pas exactement aux attentes des participants. Il est donc nécessaire d'exposer très clairement aux participants comment les résultats de la recherche seront diffusés (et utilisés) auprès d'eux-mêmes, de leur communauté et au-delà dans la société.

Enfin, les chercheurs ne doivent pas utiliser des photographies qui donneraient une image dévalorisante des participants, ou de leur communauté, notamment s'ils étaient tentés de donner un caractère «sensationnel» à leur travail de recherche.

Les considérations éthiques soulevées par l'utilisation de Photovoice sont résumées dans l'encadré 7.1. Elles doivent être discutées entre les chercheurs et les participants dès le début du projet Photovoice et peuvent être rediscutées tout au long du déroulement du projet si des clarifications s'avèrent nécessaires.

\section{Encadré 7.1. Les considérations éthiques liées à la méthode Photovoice.}

Il est important d'obtenir l'accord écrit (le consentement éclairé) de tous les participants à l'étude Photovoice, quel que soit leur âge. Cela doit être la première étape de leur participation à Photovoice.

Afin qu'il puisse utiliser les photographies pour sa recherche, le chercheur doit obtenir l'accord écrit des participants via un «formulaire d'acceptation et de décharge» détaillant les activités de recherche pour lesquelles ces photographies vont être utilisées, autorisant leur utilisation à des fins de recherche, et reconnaissant la propriété de l'auteur de la photographie.

Tous les participants doivent être conscients qu'il est indispensable de recueillir le consentement éclairé des personnes et des propriétaires des biens représentés sur les photographies. Les participants doivent recevoir les formulaires de consentement qu'ils pourront utiliser à cet effet, avant le démarrage des reportages. Ces formulaires doivent présenter le participant, le projet de recherche, l'objectif du reportage photographique, et l'usage qui sera fait des photographies. Faute d'obtenir ce consentement, ces photographies ne pourront pas être utilisées dans le cadre du projet, ni diffusées dans le cadre d'activités de «lobbying» ou plaidoyer.

Les participants doivent être conscients du nécessaire respect de la volonté des personnes de se faire photographier (ou non). Ils doivent également être respectueux des individus, des lieux et des choses qu'ils saisissent par leurs images, quel que soit leur niveau de familiarité avec ces personnes. Le chercheur doit s'assurer que les participants n'utilisent pas les photographies pour déprécier l'image de leur communauté. 
Comme les considérations éthiques, les risques potentiels (encadré 7.2) devront être discutés avec les participants dès le début du projet. Idéalement, cette discussion peut être conduite pendant l'étape de formation Photovoice et, comme pour les considérations éthiques, pourra être renouvelée tout au long du déroulement du projet.

Enfin, il est utile d'insister sur les consignes de sécurité à suivre, dès le début du projet (encadré 7.3). Ces consignes peuvent être discutées lors de la réunion entre chercheurs et participants, avant le début des prises de photographies. Dans le cas où un mode d'emploi est fourni aux participants, celui-ci pourra inclure les consignes de sécurité à titre de rappel.

\section{Encadré 7.2. Les risques potentiels associés à la méthode Photovoice.}

Les participants peuvent être stressés émotionnellement ou anxieux à l'idée d'interagir avec les personnes photographiées et de mettre en images des problèmes qu'ils vivent peut-être péniblement. Ce problème est commun à de nombreuses autres techniques de photo-élicitation et à des méthodes qualitatives plus classiques. Il est attendu une forte implication des communautés collaborant à Photovoice. De ce fait, il est possible que la mise en discussion des problèmes de la communauté et des dynamiques sous-jacentes, en particulier lorsqu'il s'agit de questions conflictuelles/litigieuses, contribue à attiser des conflits.

Il existe des risques financiers lorsque les participants ne peuvent travailler du fait du temps consacré à leur participation au projet.

Les participants doivent être informés des risques potentiels pour leur sécurité, liés aux communautés ou zones dans lesquelles ils sont susceptibles de réaliser leurs photographies.

Les participants à Photovoice peuvent aussi être victimes d'intimidation et de jugements négatifs de la part des membres de leur propre communauté qui n'ont pas été choisis pour participer au projet.

\section{Encadré 7.3. Consignes de sécurité par les utilisateurs de la méthode Photovoice.}

Votre sécurité personnelle est une priorité. Aucune photographie ne vaut la peine de vous mettre en danger.

Portez toujours un badge à votre nom ou ayez une pièce d'identité avec vous lorsque vous prenez des photos.

Restez toujours conscient de votre environnement. Par exemple, ne vous tenez pas au milieu de la route pour photographier, en particulier s'il s'agit d'une zone très fréquentée où la circulation est dense.

N'allez pas là où vous n'iriez pas habituellement et ne faites pas ce que vous ne feriez pas habituellement. Si vous vous rendez dans des zones que vous ne connaissez pas ou peu, faites-vous accompagner d'un ami ou d'une personne de confiance.

Si vous êtes agressé(e) lorsque vous prenez des photos, restez calme et ne résistez pas. Si vos agresseurs veulent prendre votre appareil photo, ne vous y opposez pas. Lappareil photo ne vaut pas la peine de mettre votre vie en danger.

Si vous avez des doutes ou des questions, adressez-vous au chercheur (ou aux assistants de terrain). 


\section{" Étude des environnements alimentaires en Afrique selon la méthode Photovoice}

Photovoice a récemment gagné en popularité dans des contextes divers et auprès des populations variées (Wang et Burris, 1997; Johnston, 2016) grâce à ce qu'elle peut apporter à tous les acteurs impliqués (participants, chercheurs, autres membres des communautés, dirigeants communautaires et décideurs politiques), comparée à d'autres méthodes plus classiques d'enquête qualitative. En pratique, elle a été utilisée pour traiter un large éventail de sujets (Johnston, 2016), par exemple la douleur chronique chez l'adulte, le vieillissement dans les établissements pour personnes âgées, la santé mentale des adolescents et plus récemment l'environnement alimentaire, et ceci essentiellement dans des pays à revenus élevés. L'application de cette méthode dans les pays à revenus faibles et intermédiaires reste limitée à quelques exemples, comme celui de l'évaluation de la vulnérabilité de la santé des communautés autochtones aux changements climatiques dans les zones rurales du sud-ouest de l'Ouganda (BerrangFord et al., 2012) ou encore l'étude des facteurs affectant l'accès aux services de santé maternelle dans la région rurale centrale de l'Ouganda (Musoke et al., 2015).

Les applications pratiques de Photovoice sont présentées ici dans le cadre de deux projets de recherche ayant mobilisé des niveaux de ressources variables (en termes de temps, moyens financiers et humains). Le premier projet porte sur les facteurs déterminant les pratiques alimentaires des habitants (hommes et des femmes âgés de 13 ans et plus) des zones urbaines défavorisées, au Kenya ( $\mathrm{n}=48$ participants $)$ et au Ghana ( $\mathrm{n}=96$ participants). Le second projet, conduit dans le cadre d'une recherche doctorale, traite des comportements alimentaires des femmes ougandaises, rurales et urbaines, en âge de procréer (FAP) ( $\mathrm{n}=18$ participantes) (Auma et al., 2020). La mise en œuvre concrète de Photovoice, les mesures prises pour l'adapter au contexte et les difficultés rencontrées dans sa mise en œuvre sont résumées dans les sections suivantes.

\section{Étape 1 : identification des communautés et publics cibles}

Dans les trois pays (Kenya, Ghana, Ouganda), les communautés ciblées pour la collecte des données ont été sélectionnées sur la base d'une analyse des données déjà disponibles et en consultant les chercheurs ayant déjà une expérience de partenariats avec les communautés locales dans ces pays. Sur la base des informations déjà disponibles, le thème de recherche a été défini suite à un pré-diagnostic des comportements alimentaires. Les communautés participantes n'ont donc pas été consultées préalablement. Au Ghana et au Kenya, le public cible pour la diffusion des résultats comprenait des membres des communautés et des acteurs de niveau local et national. En Ouganda, l'organisation d'une exposition photographique n'a pas été possible pour des raisons pratiques (recherche doctorale limitée en temps et en ressources).

\section{Étape 2 : sélection des participants à l'étude}

En Ouganda, les participants des deux sites d'étude (rural et urbain) ont été sélectionnés avec l'aide des dirigeants communautaires (agents de santé communautaires) à partir de critères établis tels que le genre (femmes) et l'âge (FAP). Les difficultés 
de recrutement des participants (et non la sélection proprement dite) ont surtout été liées à la méfiance des participants urbains quant à la manière dont les photographies seraient utilisées, si bien que le nombre de participants prenant des photographies a été moins élevé que souhaité. Les participants des zones rurales n'ont pas posé ce genre de difficultés. Comme en Ouganda, les participants kenyans et ghanéens ont été sélectionnés avec l'aide des dirigeants communautaires à partir de critères préétablis tels que le genre, l'âge, la situation professionnelle, la catégorie d'indice de masse corporelle (IMC) et la situation en matière de grossesse ou d'allaitement des FAP.

\section{Étape 3 : session de formation et d'échange proposée aux participants}

En Ouganda, une formation à Photovoice a été offerte aux participants ruraux et urbains. Dans la zone rurale ougandaise, cette formation a pris la forme d'une unique séance de groupe de plus d'une heure à laquelle ont assisté tous les participants. Dans les sites urbains d'étude des trois pays (Ghana, Kenya et Ouganda), elle a été menée individuellement. Les restrictions en matière de temps et de ressources ont rendu impossible la réunion de tous les participants urbains pour une séance groupée de formation à la méthode, comme celle proposée à leurs homologues ruraux. Un manuel de formation à Photovoice a été préparé pour orienter la réunion dans les deux contextes, rural et urbain, et traduit dans les langues locales.

\section{Étape 4 : consentement/assentiment éclairé}

Tous les participants à l'étude, en Ouganda, au Kenya et au Ghana, ont donné leur consentement éclairé et un assentiment éclairé a été obtenu pour les participants de moins de 18 ans. En plus des formulaires de consentement éclairé, des formulaires supplémentaires de consentement ont été préparés pour les participants relativement au «consentement éclairé pour les personnes et propriétés privées représentées dans les photographies", ainsi qu'un «formulaire d'acceptation et de décharge» autorisant le chercheur à utiliser les photographies des participants. Les participants ruraux ont évoqué des difficultés à obtenir le consentement des membres de leurs foyers avant de les photographier. Par exemple, le fait de demander le consentement des maris pouvait être perçu comme irrespectueux. Pour surmonter ce problème, certains participants ont choisi de prendre des photos ne permettant pas d'identifier les membres de leurs foyers, ce qui a rendu inutile l'obtention d'un consentement.

\section{Étape 5 : proposition du thème de recherche}

Dans les trois pays (Ghana, Kenya et Ouganda), le thème de recherche a été défini avant la réunion initiale avec les participants, après une analyse documentaire menée pour identifier les problèmes de la population cible.

\section{Étape 6 : reportage photographique}

En Ouganda, il a été demandé aux participants de prendre cinq photographies illustrant cinq thèmes (à savoir : ce que signifie pour eux la nourriture; où ils se procurent 
leur nourriture; avec qui ils mangent; où ils mangent et comment ils préparent les aliments). Ils pouvaient aussi prendre des photos supplémentaires s'ils pensaient que cela éclairerait davantage leurs récits photographiques. Chaque participant a reçu un guide (photographique) résumant les objectifs du projet et les cinq thèmes qu'il fallait illustrer avec les photographies, ainsi que les considérations éthiques et les risques éventuels. Au Kenya et au Ghana, les cinq thèmes que devaient couvrir les photographies des participants étaient les suivants : quelque chose dans votre environnement immédiat qui influe sur ce que vous mangez; quelqu'un dans votre environnement immédiat qui influe sur ce que vous mangez; un endroit où vous mangez; quelque chose qui vous permet de manger sainement et quelque chose qui vous empêche de manger sainement. Dans ces deux projets reposant sur la méthode Photovoice (Ouganda, Kenya, Ghana), les reportages ont été réalisés sur une semaine. L'équipe de recherche a donc été assez directive (contrairement à d'autres projets, où les thèmes proposés aux participants sont plus larges).

\section{Étape 7 : analyse des photographies}

En Ouganda, au Kenya et au Ghana, les photographies ont été analysées au cours d'entretiens approfondis semi-structurés plutôt que dans des discussions de groupe Photovoice. L'alimentation étant un sujet très chargé émotionnellement et révélant un important attachement socioculturel dans le cadre de cette étude, il a semblé que les participants se sentiraient sans doute plus à l'aise d'analyser les photographies, (qui représentaient des fenêtres ouvertes sur leurs vies) en face-à-face avec le chercheur plutôt qu'en groupe. L'analyse en groupe des questions portant sur les comportements alimentaires et des facteurs les influençant auraient pu susciter des sentiments de gêne ou une stigmatisation, d'autant plus que les participants venaient de communautés dont les membres sont très proches. En outre, il aurait été difficile de réunir les participants ougandais urbains dans un même lieu pour analyser les photographies.

\section{Étape 8 : diffusion des résultats}

\section{Diffusion par la médiatisation des résultats de la recherche}

Diverses stratégies de diffusion ont été employées en Ouganda, au Kenya et au Ghana. Dans ces deux derniers pays, une exposition photographique illustrant les déterminants des pratiques alimentaires a été organisée dans un lieu public de chacune des villes participantes (Ho, Accra et Nairobi) en vue de sensibiliser les communautés et les médias aux facteurs déterminant la consommation d'aliments et boissons denses en calories et pauvres en nutriments. Un large éventail d'acteurs a visité ces expositions : membres des communautés locales, ONG, représentants des institutions gouvernementales (ministères de la Santé, de l'Alimentation et de l'Agriculture), des services sanitaires et des gouvernements locaux, et médias (y compris des radios publiques et privées, la presse écrite et les chaînes de télévision). Au Ghana et au Kenya, les résultats ont aussi été diffusés aux acteurs nationaux par le biais de réunions de mobilisation des parties prenantes, organisées à la fin du projet et durant lesquelles étaient distribués des 
fascicules avec les photographies réalisées. L'objectif était de stimuler le débat sur les types de problèmes rencontrés dans leur vie quotidienne par les membres des communautés pour manger sainement. Des propositions d'interventions et de politiques visant à résoudre ces problèmes ont été présentées par l'équipe de recherche et débattues avec les décideurs politiques. En Ouganda, parce que l'étude a été réalisée dans le cadre d'un projet de doctorat, les fonds ont manqué pour assurer la diffusion des résultats immédiatement après la collecte et l'analyse des données. Depuis, un plan de diffusion visant une mobilisation publique plus importante a toutefois été formulé.

\section{Diffusion par des publications scientifiques}

Les résultats des études des trois pays sont également en cours de diffusion dans le milieu universitaire (Auma et al., 2020; Pradeilles et al., à paraître). À cet effet, les données ont été analysées au moyen d'une liste de codes thématiques permettant d'étiqueter les données collectées. L'approche adoptée pour le développement du schéma de codage et de l'analyse subséquente était à la fois théorique, puisqu'elle utilisait des thèmes préexistants compilés à partir d'un modèle socio-écologique antérieur de comportements alimentaires (Story et al., 2008) et fondée sur les données (c'est-à-dire sur les thèmes émergeant des données pendant l'enquête). Le modèle socio-écologique illustre des facteurs multiples au niveau individuel et environnemental (réseaux sociaux, environnement physique et macro-environnement) qui influent directement ou indirectement sur ce que les gens consomment (Story et al., 2008). Pour le projet de doctorat en Ouganda, par exemple, les codes issus des données utilisés dans l'analyse des environnements physiques des participants comprenaient entre autres des mots ou expressions tels que «frigo », «cuisinière », «potager familial », «près», «loin», «lieu de travail», «argent». Une fois tous les récits et photographies des participants encodés, les codes ont été regroupés sous plusieurs sous-thèmes et thèmes. Par exemple, les codes «près», «loin» ont été regroupés sous le sous-thème «accès physique aux établissements alimentaires», les codes «frigo », «cuisinière», «potager familial» sous le sous-thème «disponibilités alimentaires du ménage » et les codes «argent», «coût » sous le sous-thème «accès financier ». Les sous-thèmes «accès financier », «frelatage des aliments», «accès physique aux établissements alimentaires», «type d'aliments disponibles» (dans le foyer ou la communauté/le quartier), «type d'établissement alimentaire» ont alors été collectivement regroupés sous le thème général «environnement physique».

À partir du récit du participant, la figure 7.1, par exemple, a d'abord été encodée en utilisant le mot «près». Ensuite, cette même photo a été encodée sous le sous-thème «accès physique aux établissements alimentaires » et sous le thème «environnement physique».

Dans la figure 7.2, par contre, la photographie a été encodée en utilisant les mots «argent», «coût», «matériel», qui ont ensuite été regroupés sous le sous-thème «accès financier», puis sous le thème «environnement physique», parce que le coût et la disponibilité étaient propres au contexte dans lequel les participants étaient placés et reflétaient ainsi la dynamique de «l'environnement physique»-que ce soit le foyer, l'école ou le quartier dans lequel ils vivaient. 


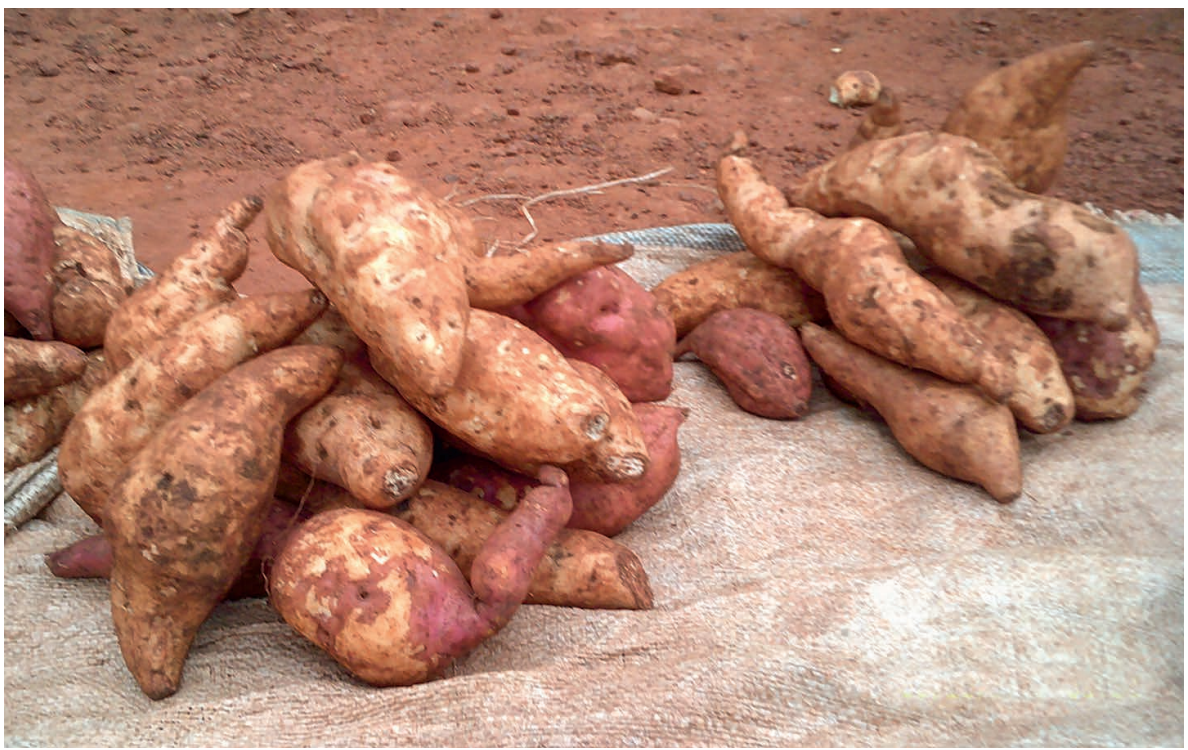

Figure 7.1. Étal de patates douces sur un marché (participant 17$)^{31}$.

«Les patates douces sont l'aliment qui, pour nous, est le plus facilement disponible à proximité, et donc c'est ce que nous mangeons habituellement. De plus, à l'étal où nous achetons notre nourriture, c'est l'aliment qui est proposé. Ils ne vendent pas d'autres produits, comme du riz. Tout ce qu'elle a, ce sont des patates douces et du matooke» (participant 17, résident rural, catégorie d'âge 15-17 ans).

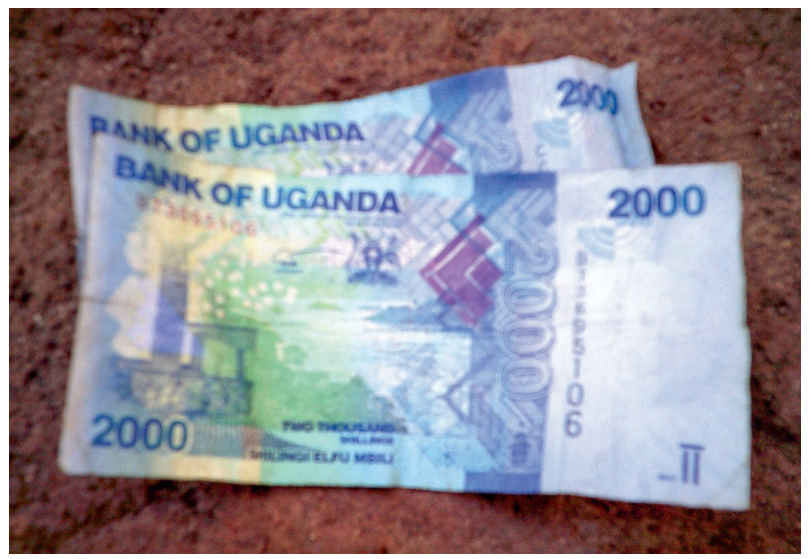

Figure 7.2. Billets de banque ougandais (participant 11).

«Si j'ai de l'argent, je mange "ma nourriture". Sinon, je mange juste pour la satisfaction ou comment dire... le facteur de satisfaction» (participant 11, résident rural, catégorie d'âge 18-34 ans).

Dans le dernier exemple (figure 7.3), la photographie a d'abord été encodée en utilisant les mots «potager familial», «agriculture urbaine», «matériel». Ces mots ont été regroupés sous le sous-thème «disponibilités alimentaires du ménage» et ensuite sous le même thème «environnement physique».

31. Les images présentées sont des photos prises par les participants à l'enquête. 


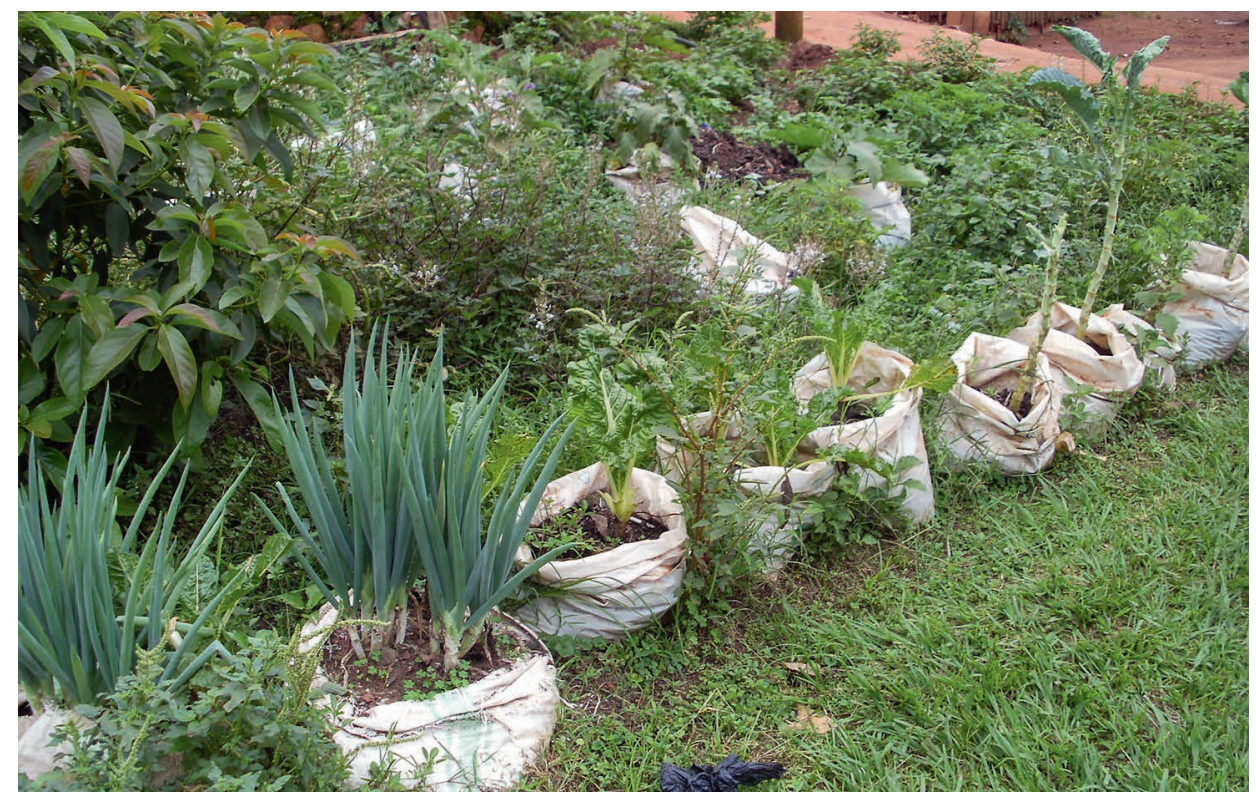

Figure 7.3. Potager familial en milieu urbain (participant 5).

«Ce sont des légumes verts... des oignons. Nous utilisons toujours les feuilles des oignons. Il y a le nakatti ici, et le dodo là... Là, c'est le sukuma wiki et l'autre... j'ai oublié son nom. Il y a tout là... Lavoine est là, dans ce petit jardin $k a$ que vous voyez» (participant 5, résident urbain, catégorie d'âge 35-49 ans).

Après l'encodage, les liens entre thèmes et sous-thèmes ont été examinés parallèlement aux photos.

\section{Étape 9 : dynamique du changement social enclenché}

Il est trop tôt pour relever les impacts de ces projets sur tous les sites d'étude concernés (Kenya, Ghana et Ouganda), car les travaux sur le terrain se sont terminés récemment, en 2019. Les résultats ayant été diffusés aux communautés et décideurs politiques au Ghana et au Kenya, il est attendu que le projet mobilise les politiques et les décideurs pour lever les obstacles et retenir des solutions pour permettre à ces communautés à faibles revenus d'accéder à une alimentation saine.

Pour conclure ce chapitre, Photovoice peut être utilement mobilisée pour comprendre le rôle des environnements alimentaires sur les comportements nutritionnels; elle va au-delà des approches classiques, qui visent généralement à corréler ces comportements à des caractéristiques «intrinsèques» individuelles. Elle permet de se concentrer sur la façon qu'ont les individus d'appréhender leur environnement et d'interagir avec lui. Par ailleurs, un autre aspect de Photovoice a été révélé par sa mise en œuvre au Ghana. Alors que le projet visait à étudier les problèmes nutritionnels liés à l'environnement alimentaire dans les villes ghanéennes, les participants ont mis en avant à travers leur récit d'autres questions de sécurité alimentaire qu'ils jugeaient prioritaires. En donnant la parole aux membres des communautés et en 
leur permettant d'exprimer ce qui influence leur alimentation, le processus a permis de recadrer l'objet central du projet initial des chercheurs. Cela révèle le fossé qui peut parfois exister entre les préoccupations des acteurs du développement, des chercheurs (ici, des spécialistes de la nutrition) et des citoyens. Ces observations sur la sécurité alimentaire permettent d'orienter les interventions et les politiques des décideurs. Les images produites avec Photovoice pourraient constituer, au-delà des mots, un puissant levier de mobilisation en ce sens.

\section{Références}

Auma C. I., Pradeilles R., Blake M. K. B., Musoke D., Holdsworth M., 2020. Factors influencing dietary practices in a transitioning food environment: a cross-sectional exploration of four dietary typologies among rural and urban Ugandan women using Photovoice. Nutrition Journal, 19 (127), 1-15. https://doi.org/10.1186/s12937-020-00634-9

Berrang-Ford L., Dingle K., Ford J. D., Lee C., Lwasa S., Namanya D. B., Henderson J., Llanos A., Carcamo C., Edge V., 2012. Vulnerability of Indigenous Health to Climate Change: A Case Study of Uganda's Batwa Pygmies. Social Science \& Medicine, 75 (6), 1067-1077.

https://doi.org/10.1016/j.socscimed.2012.04.016

Catalani C., Minkler M., 2010. Photovoice: A Review of the Literature in Health and Public Health. Health Education \& Behaviour, 37 (3), 424-451. https://doi.org/10.1177/1090198109342084

Johnston G., 2016. Champions for Social Change: Photovoice Ethics in Practice and "False Hopes" for Policy and Social Change. Global Public Health, 11 (5-6), 799-811.

https://doi.org/10.1080/17441692.2016.1170176

Musoke D., Ekirapa-Kiracho E., Ndejjo R., George A., 2015. Using Photovoice to Examine Community Level Barriers Affecting Maternal Health in Rural Wakiso District, Uganda. Reproductive Health Matters, 23 (45), 136-147. https://doi.org/10.1016/j.rhm.2015.06.011

Nykiforuk C. I. J., Vallianatos H., Nieuwendyk L. M., 2011. Photovoice as a Method for Revealing Community Perceptions of the Built and Social Environment. International Journal of Qualitative Methods, 10 (2), 103-124. https://doi.org/10.1177/160940691101000201

Pradeilles R., Irache-Ezpeleta A., Njeri M., Holdsworth M., Laar A., Zotor F., Muthuri S., KimaniMurage E., Graham F., Green M., Osei-Kwasi H., Tandoh A., Klomegah S., Nathaniel C., Bohr M., Griffiths P., 2021. Urban physical food environments drive dietary behaviours in Ghana and Kenya: a Photovoice study. Health and Place, 71. https://doi.org/10.1016/j.healthplace.2021.102647

Story M., Kaphingst K. M., Robinson-O'Brien R., Glanz K., 2008. Creating Healthy Food and Eating Environments: Policy and Environmental Approaches. Annual Review of Public Health, 29 (1), 253-272. https://doi.org/10.1146/annurev.publhealth.29.020907.090926

Wang C. C., Burris M. A., 1994. Empowerment through Photo Novella: Portraits of Participation. Health Education Quarterly, 21 (2), 171-186. https://doi.org/10.1177/109019819402100204

Wang C. C., Burris M. A., 1997. Photovoice: Concept, Methodology, and Use for Participatory Needs Assessment.HealthEducation \& Behaviour, 24(3),369-387.https://doi.org/10.1177/109019819702400309

Wang C. C., 1999. Photovoice: A Participatory Action Research Strategy Applied to Women's Health. Journal of Women's Health, 8 (2), 185-192. https://doi.org/10.1089/jwh.1999.8.185

Wang C. C., Redwood-Jones Y. A., 2001. Photovoice Ethics: Perspectives from Flint Photovoice. Health Education \& Behaviour, 28 (5), 560-572. https://doi.org/10.1177/109019810102800504 


\title{
Chapitre 8 \\ Les narrations quantifiées : une méthode qui combine entretien et analyse statistique des dynamiques biographiques
}

\author{
GrÉGORI AKERMANN ${ }^{a}$ et PAUL COEURQUETIN ${ }^{b}$
}

a INRAE, UMR Innovation, 34060 Montpellier, France.

${ }^{\mathrm{b}}$ Université de Toulouse Jean-Jaurès, LISST - CNRS, France.

Cette méthode mixte, qualitative et quantitative, vise à comprendre l'engagement des mangeurs dans des pratiques alimentaires en procédant à une description fine des étapes et des séquences d'accès à des ressources. Elle consiste d'abord à recueillir des données qualitatives issues d'entretiens semi-directifs auprès de personnes concernées par les pratiques alimentaires étudiées, puis à rédiger un récit détaillé qui fera enfin l'objet d'une codification et d'une analyse statistique, ce qui permet de comparer et de construire une typologie des parcours d'engagement.

La méthode des narrations quantifiées trouve son ancrage théorique dans la sociologie des réseaux sociaux. Celle-ci s'intéresse aux relations sociales, aux liens qui s'établissent entre des personnes, des organisations ou des groupes, ainsi qu'aux réseaux que constituent ces relations (Degenne et Forsé, 1994). Elle peut s'intéresser aux «réseaux sociaux numériques» ou aux «médias sociaux» tels que Twitter ou Facebook, en posant des questions spécifiques sur la nature des liens médiés par les dispositifs numériques ou encore en mobilisant ses outils mathématiques pour étudier la structure des réseaux formés par les liens entre les utilisateurs de tel ou tel outil, mais elle ne doit pas être confondue avec une sociologie du numérique, qui s'intéressera quant à elle aux usages ou aux identités numériques. La sociologie des réseaux sociaux peut-être découpée en trois grands ensembles de recherches, qui approchent les réseaux et les relations de manières différentes. Un premier ensemble comprend les études de réseaux complets, qui examinent la structure des liens unissant un ensemble défini d'acteurs (tous les membres d'une entreprise ou toutes les entreprises d'un secteur par exemple). Les analyses de réseaux complets sont souvent centrées sur la question du pouvoir (qui se trouve dans la meilleure position?) ou sur la cohésion (le réseau est-il plus ou moins fragmenté ?). Un deuxième ensemble se compose des études de réseaux personnels, qui enquêtent sur un échantillon classique 
de personnes afin de reconstituer, pour chaque enquêté, les relations directes que celui-ci entretient avec d'autres personnes, et les relations que les personnes de son entourage entretiennent entre elles. Les déterminants de la structure des relations et des réseaux peuvent ensuite être recherchés, de même que leur évolution dans le temps en fonction d'événements biographiques. Enfin, le troisième ensemble inclut l'étude des chaînes relationnelles, qui cherche quant à elle à suivre les ressources circulant au travers des relations dans des réseaux non délimités. Certaines études se centrent sur les problématiques de la diffusion - afin de connaître la taille théorique d'un réseau (étude du petit monde) - lorsque d'autres cherchent à comprendre les processus d'accès aux ressources (accès à l'emploi, à des contrats, à des informations...).

La méthode des «narrations quantifiées » s'inscrit dans ce troisième groupe d'études, qui cherchent à comprendre le rôle des relations sociales et des dispositifs dans des processus «entrepreneuriaux», c'est-à-dire visant à transformer l'état du monde au moyen d'une suite d'activités (Chauvin et al., 2014). Elle a été élaborée et expérimentée pour la première fois dans une recherche sur les relations sciences-industrie menée par Michel Grossetti et Marie-Pierre Bès. La méthode a par la suite été reprise et adaptée pour étudier la création de start-up, l'économie informelle au Burkina Faso, la création d'entreprises accompagnées, l'accès à l'emploi, les inventeurs autonomes, la création d'entreprises en sciences humaines et sociales. Elle a enfin été formalisée dans deux articles méthodologiques (Grossetti 2011; Grossetti et al., 2011) et a récemment fait l'objet d'une adaptation pour l'étude des trajectoires de personnes malades de longue durée (Akermann et al., 2018) et enfin pour l'étude des trajectoires d'éviction du gluten (Akermann et Coeurquetin, à paraître).

La méthode des narrations quantifiées est une méthode mixte qui combine un recueil de données qualitatives par entretiens semi-directifs auprès de plusieurs personnes concernées par l'activité étudiée, un recueil de données secondaires (documents, fichiers numériques...), la rédaction d'un récit par le chercheur qui peut être envoyé aux enquêtés, une étape de codification des récits et d'analyse statistique d'une base de données des séquences d'accès à des ressources. Elle a pour objectif de comprendre un processus social en procédant à une description fine des étapes et des séquences d'accès à des ressources (par exemple, une information, un conseil, un matériel, une prestation de service, un financement, un soutien émotionnel ou instrumental) qui ont orienté l'action des personnes. Elle a été conçue pour étudier la problématique de l'encastrement de l'activité (en particulier économique) dans les réseaux de relations sociales (Granovetter, 1985). En donnant la possibilité de calculer, à plusieurs étapes, la part des relations sociales activées pour mener à bien une activité économique, elle a permis de mettre au jour les processus d'autonomisation progressive des activités visà-vis des relations sociales dont elles émergent (Grossetti et Barthe, 2008).

\section{A Appréhender ce que font les mangeurs et les contextes de leurs actions}

Comme beaucoup d'autres méthodes utilisées dans les sciences sociales, la méthode des narrations quantifiées n'a pas été conçue pour étudier spécifiquement l'alimentation et les mangeurs. En tant que méthode d'analyse des processus sociaux, elle 
permet - après quelques adaptations - d'étudier des processus qui concernent les pratiques alimentaires des mangeurs ou des pratiques collectives, comme l'approvisionnement au sein de groupements d'achats. Positionnée à l'échelle individuelle, elle s'avère ainsi très utile pour analyser les changements de comportements alimentaires, en particulier lorsque les consommateurs adoptent une posture active, c'est-à-dire lorsqu'ils mettent en œuvre une succession d'activités pour changer leur alimentation, la manière de s'approvisionner ou de cuisiner. Entrée dans une consommation «zéro déchet», «locavore», dans de nouvelles formes d'approvisionnement collectif ou collaboratif, une production «autonome», une mise en pratique d'un régime alimentaire sans gluten, sans additifs, crudivore, comprenant des aliments lactofermentés, nécessitant de rechercher des produits alimentaires rares, etc. : la méthode des narrations quantifiées permet d'analyser les trajectoires individuelles de consommateurs étant entrés, volontairement ou non (pour raisons médicales par exemple), dans une phase active de modification de leurs pratiques alimentaires, voire de bifurcation alimentaire. Positionnée à une échelle collective, la méthode est tout à fait adaptée à l'étude de changements collectifs (par exemple familiaux) ou à l'étude de la structuration de groupes de consommateurs entreprenant collectivement la création d'une activité de production (jardin collectif...) ou de distribution (groupement d'achats, Amap [Association pour le maintien d'une agriculture paysanne], etc.). Il s'agit alors d'analyser la manière dont une activité à vocation de production ou de consommation s'ancre dans des systèmes d'acteurs territoriaux et dans les réseaux qui entourent les mangeurs-entrepreneurs ${ }^{32}$.

Guidé par l'enquêteur, l'enquêté est invité à raconter l'histoire de son changement de pratique ou de la création d'une activité collective en retraçant toutes les étapes qui l'ont conduit d'un état initial à un état final dans lequel son régime ou ses pratiques alimentaires ont été modifiés et se sont stabilisés. Dans les récits, qui s'étendent d'une durée de quelques mois à quelques années, guidés par les relances précises de l'enquêteur, les personnes sont invitées à indiquer les supports de leurs actions : informations sur l'alimentation en général ou sur un produit, un matériel, un lieu de vente en particulier, les conseils culinaires ou de santé reçus, les nouveaux matériels, nouveaux produits, nouveaux dispositifs de qualification ou de vente, nouvelles manières de cuisiner les denrées qui s'offrent à lui. Chaque information, chaque conseil, chaque produit ou matériel, signalés par la personne dans son récit, sont considérés comme une «ressource», autrement dit un support de l'action, qui circule au travers d'un mode d'accès, qui peut-être une relation interpersonnelle, un professionnel, un organisme, une institution, un espace marchand, un média, etc. La méthode permet d'éviter l'écueil d'une individualisation des processus qui ne s'attacherait qu'à la description des compétences individuelles de la personne, voire d'une psychologisation du processus, qui prendrait pour objet les liens entre l'activité menée et les traits de personnalité. L'analyse des ressources mobilisées fait nécessairement apparaître les collectifs, les institutions, les dispositifs, les réseaux et les autrui significatifs qui prennent place dans le processus de création d'activités ou de changement de pratiques. La question centrale à laquelle la méthode permet de répondre est donc : quels sont les ingrédients de l'action?

32. Nous appelons «mangeur-entrepreneur» un individu cherchant à modifier l'état de ses pratiques alimentaires ou l'état du système alimentaire dans lequel il est inséré, c'est-à-dire entrant dans une bifurcation alimentaire active, individuelle ou collective. 
L'analyse des modes d'accès montre la diversité des acteurs individuels et collectifs, met en lumière les prescripteurs et plus largement les mondes sociaux qui influencent ou soutiennent les représentations et les pratiques à différentes étapes de la trajectoire individuelle ou collective. La diversité des relations signalées dans les récits, la modification des types de relation, de la fréquence, de la force des liens, la création de nouvelles relations pendant le parcours, ouvrent aussi la possibilité d'entrer finement dans l'analyse des dynamiques relationnelles qui accompagnent les changements de pratiques alimentaires. Changer de pratiques alimentaires, est-ce également changer d'entourage, et inversement?

En croisant l'analyse des moments d'acquisition et des modes d'accès aux ressources, il est possible d'identifier des dynamiques d'autonomisation ou de dépendance, dans le temps, vis-à-vis de certains mondes (monde médical, marchand, numérique...) et de certains cercles relationnels (familial, amical, professionnel...). Le monde médical influence-t-il les consommateurs tout le long de leur trajectoire ? À quelles étapes de la trajectoire les réseaux sociaux numériques, les membres de la famille, les espaces marchands influencent-ils le plus les consommateurs? Quels sont les cercles relationnels qui accompagnent les changements de pratiques alimentaires ou la création d'un groupement d'achats de consommateurs? Quels sont les outils, les méthodes et les recettes qui accompagnent un changement de pratiques culinaires?

Lorsque l'on positionne l'analyse à l'échelle d'un échantillon de plusieurs dizaines d'individus, il est possible d'identifier les récurrences de certaines trajectoires, d'expliquer certaines dynamiques par la position sociale des personnes, par la structuration des espaces dans lesquels les acteurs se situent. Les personnes plus jeunes ou plus âgées utilisent-elles les mêmes types de ressources pour guider leurs actions? Les consommateurs urbains et ruraux s'appuient-ils sur les mêmes ressources, les mêmes types de lieux de vente, les mêmes types de professionnels (monde médical, du sport, du bien-être...), les mêmes savoirs et les mêmes outils?

Lorsque les trajectoires étudiées se situent dans le même contexte historique, géographique ou social, il arrive souvent de repérer des ressources partagées par plusieurs personnes ou par des collectifs ayant des trajectoires pourtant différentes (un livre cité par plusieurs personnes, un médecin, une association, un lieu de vente, un matériel, un producteur...). Si les trajectoires se retrouvent interconnectées au travers de ces ressources partagées, ce sont aussi des réseaux qui se dessinent et qui font apparaître des intermédiaires. La présence ou l'absence de ces acteurs centraux peut devenir un indicateur du niveau de structuration, voire d'institutionnalisation d'un mouvement collectif de changement de pratiques alimentaires, de réappropriation de son alimentation. Les personnes qui se mettent à consommer sans gluten s'appuient-elles sur les mêmes rationalités diffusées par les mêmes ouvrages, les mêmes blogs, les mêmes groupes sur les réseaux sociaux? Qui sont les promoteurs du «zéro déchet», quels savoirs diffusent-ils et ont-ils un impact sur les pratiques des consommateurs? Quelles institutions soutiennent la création de potagers collectifs ou de groupements d'achats citoyens?

La méthode des narrations quantifiées s'adapte très bien à l'étude d'acteurs individuels ou de collectifs en situation d'entreprendre une activité liée à l'alimentation (production, distribution, approvisionnement) ou un changement de pratique alimentaire (régime alimentaire spécifique, mode d'approvisionnement, de conservation, 
manière particulière de cuisiner) sur une période délimitée dans le temps. Elle se prête également très bien à l'analyse de périodes bornées (de quelques mois à quelques années), ponctuées d'événements qui créent des incertitudes, qui remodèlent les représentations, qui modifient les contextes d'action et qui ont pour conséquences d'activer des séquences d'accès à des ressources. Sans événement dans les trajectoires de consommation des acteurs, sans l'intervention d'acteurs identifiables, sans apparition d'innovations, les récits sont plus flous : les personnes manquent de repères biographiques et temporels pour engager et dérouler le récit.

Du fait qu'elle fait appel à la mémoire des acteurs, la méthode des narrations quantifiées est en revanche peu adaptée à l'étude de changements latents, moins conscients, dans lesquels les acteurs restent passifs. À moins de se positionner sur une échelle très courte (de l'ordre de la journée), la méthode est donc peu adaptée à l'étude des périodes de consommations routinières pendant lesquelles les changements sont marginaux. La méthode oriente volontairement les récits sur les supports de l'action plutôt que sur les rationalités des acteurs. Si demander aux personnes de porter leur attention aux éléments exogènes qui orientent leurs pratiques ne les empêche pas d'aborder, pendant l'entretien, leurs théories, leurs rationalités et leurs représentations, il s'agit d'une méthode moins centrée sur ce que les acteurs pensent que sur ce que les acteurs font et le contexte de leurs actions.

\section{W Aspects éthiques et déontologiques}

La méthode des «narrations quantifiées» ne pose pas de questions éthiques, juridiques ou déontologiques supplémentaires à celle de la collecte de données qualitatives (par entretiens) et quantitatives (par questionnaires) en sciences humaines et sociales. Appliquée aux trajectoires individuelles en matière de consommation et d'alimentation, une certaine éthique de la recherche à l'égard du recueil des informations et de leur traitement est à encourager compte tenu de la proximité du fait alimentaire avec des dimensions intimes, médicales et religieuses notamment. Appliquée aux mêmes domaines, mais à l'analyse de trajectoires collectives et d'organisation - où les vecteurs de ressources peuvent être les mêmes pour un grand nombre d'acteurs, ou géographiquement proches, ou encore en concurrence -, la vigilance sur les données devient alors encore plus essentielle et s'étend jusqu'à la restitution aux enquêtés.

Ainsi, de manière spécifique à cette méthode d'enquête (quelle qu'en soit l'échelle), mais de façon commune à l'analyse des réseaux sociaux, les bases de données que les chercheurs exploitent contiennent d'importantes quantités d'informations sur d'autres individus que les personnes interrogées (liens entre deux personnes, types de relations, profession, proximité géographique, etc.). En raison de cette démultiplication des données à caractère personnel et des possibilités d'identification par croisement qui s'en trouvent augmentées, ces bases de données imposent un besoin plus prégnant de conformité vis-à-vis du règlement général de protection des données (RGPD) en ce qui concerne l'anonymisation des données et leur sécurité d'un bout à l'autre du travail d'enquête, d'analyse et donc d'information des publics enquêtés, de partage des données entre chercheurs, de valorisation scientifique et d'archivage informatique. 


\section{" Une méthode applicable à des échelles variées et utilisées par différentes disciplines}

La méthode des narrations quantifiées peut être utilisée à des échelles variées. Elle s'adapte à l'analyse de trajectoires individuelles, de trajectoires collectives (familiales, groupe de citoyens), de trajectoires d'organisation (Amap, association, épicerie...) ou de trajectoires de projets multipartenariaux. Elle peut être mise en œuvre à des échelles temporelles courtes (de l'ordre de la journée) ou longues (plusieurs années) et peut même être utilisée de manière longitudinale. Il s'agit d'une méthode mixte, couplant recueil de données qualitatives et analyse statistique, et elle permet de faire de l'analyse de chaînes relationnelles et, dans certaines conditions, de l'analyse de réseaux. Les données qualitatives issues des entretiens laissent aux enquêtés la possibilité de s'exprimer sur le sens que prennent leurs actions. La rédaction du récit formalise la structure des trajectoires individuelles ou collectives et met en lumière les étapes et les points de bifurcations. Les analyses statistiques de la base de données mettent à distance les individus pour dévoiler les récurrences dans les processus, des déterminants sociodémographiques, géographiques ou institutionnels. Les analyses de réseaux mettent au jour des acteurs centraux, des intermédiaires, la structure d'un système d'acteurs ou des chaînes de relations.

Bien qu'issue de la sociologie, la méthode est d'ores et déjà utilisée par des économistes et des géographes qui la mettent en œuvre pour étudier le rôle de la proximité géographique dans la mise en ouvre et la réussite de projets innovants, l'efficacité des dispositifs institutionnels dédiés à leur accompagnement, ainsi que l'impact du contexte territorial (métropoles, villes moyennes, espace rural...). De cette façon, en se focalisant sur les «ressources» (cognitives, économiques, instrumentales, émotionnelles), la méthode peut intéresser toutes les disciplines du champ des sciences humaines. Cette méthode pourrait venir éclairer les analyses en nutrition et santé publique sur les trajectoires de changement de régime alimentaire : bifurcation vers un régime végane, végétarien, flexitarien, etc.

\section{Un cas d'application à la pratique des régimes «sans gluten»}

Pour illustrer un cas d'utilisation de la méthode des «narrations quantifiées » pour l'analyse de changements alimentaires, nous présentons une enquête réalisée durant l'été 2018, menée auprès de personnes se déclarant «sensibles au gluten non coeliaques » (SGNC). Cette enquête a été conduite dans le cadre du projet «Gluten, mythe ou réalité ?»: une recherche pluridisciplinaire visant à caractériser la potentielle meilleure digestibilité de certains produits céréaliers au regard de plusieurs facteurs (variétés, systèmes, procédés de transformation et de fabrication) ${ }^{33}$. En vue

33. Le projet «Gluten, mythe ou réalité ? » est soutenu par la Fondation de France, la Fondation Édouard et Geneviève Buffard et par la Fondation Daniel et Nina Carasso. Kristel Moinet (animatrice du Biocivam de l'Aude) et Dominique Desclaux (chercheuse de l'INRAE) coordonnent ce projet en collaboration avec plusieurs équipes de l'INRAE et de nombreux acteurs de terrain : agriculteurs, transformateurs, acteurs du conseil agricole et médecins. 
d'apporter un éclairage sur les motivations, les pratiques d'achats, les habitudes alimentaires de ces personnes, mais surtout les ressources qui constituent des points d'appui pour leur transition vers un régime restrictif, le travail sociologique mené s'est positionné en faveur d'une étude des trajectoires médicales et de consommation. C'est justement en envisageant le parcours de ces personnes comme une succession d'étapes - les amenant progressivement (ou rapidement) du diagnostic à l'éviction, puis vers la consommation de ces produits céréaliers considérés comme plus digestes - que nous avons fait le choix d'étudier les aspects sociologiques du «devenir », puis du «être» sensible au gluten.

La méthode combine un recueil de données par entretiens semi-directifs et une méthode de codification des récits : il s'agit donc, dans un premier temps, de construire un récit à partir d'entretiens avec les personnes se déclarant SGNC. Ces entretiens sont à trame biographique (Bertaux, 1997) et insistent, à l'aide de relances systématiques, sur le recueil d'éléments qui concernent l'accès aux ressources. Ces relances précises sont nécessaires pour identifier le plus finement possible les types de ressources, les moyens mobilisés pour accéder à la ressource (chaîne de relations ou formes de médiations) et, le cas échéant, le type de relation entre les individus qui interviennent dans cette chaîne.

Dans le cas de ce projet de recherche, il s'agissait de retracer l'itinéraire des personnes sensibles au gluten comme un processus continu de séquences d'accès à des informations, des soins, des tests, etc. - depuis la survenue de leurs symptômes jusqu'à la résolution de leurs problèmes - en recueillant des informations sur l'ensemble des ressources servant de support à l'action (recommandation d'arrêt du gluten, recommandation d'un médecin, informations sur les produits céréaliers, sur l'alimentation industrielle ou artisanale, etc.), ainsi que leurs vecteurs (les médecins, les personnes proches, les livres, les sites Internet, etc.).

Concrètement, nous proposions aux enquêtés de raconter ce qui les avait conduits à retirer le gluten de leur alimentation. Nous cherchions alors à les guider dans les différentes périodes à la fois empiriques et conceptuelles qui nous servaient ainsi de trame pour la conduite de l'entretien (errance diagnostique, diagnostic, régime sans gluten strict, dilettante ou sélectif). À ce sujet, les premiers entretiens à teneur exploratoire se sont avérés utiles pour bien repérer les différentes étapes, les ressources qui y sont obtenues et ce qu'elles induisent sur le reste du parcours de soins ou, de manière plus générale, sur les processus de changements alimentaires qui peuvent se prêter à cette méthodologie d'enquête.

Une fois l'entretien réalisé, le chercheur est amené à en faire un compte rendu sur la base de ses notes et de ses enregistrements audios. Ce compte rendu peut être enrichi par des entretiens avec d'autres personnes concernées par le processus étudié (membre de la famille, producteurs, médecins...) et par la consultation de documents (trace numérique de l'activité, ticket de caisse, carte de visite, note d'agenda...). L'objectif est ici de rédiger un récit chronologique, relativement épuré, mais aussi le plus précis possible. Le récit peut alors être éventuellement envoyé aux enquêtés rencontrés en cas de doute sur certaines informations, pour validation, voire ajouts. Ensuite, le chercheur doit repérer dans le récit les séquences d'accès aux ressources (tableau 8.1). Pour chaque séquence - qui prend la forme de quelques lignes descriptives dans le récit -, le chercheur code un certain nombre 
de variables, telles que le mode d'accès, le type de ressource, le type de relation ou de médiation, et la date de mobilisation (en années, voire en mois). Une fois le récit codé, il est possible de procéder à une analyse quantitative des séquences pour étudier les hypothèses formulées.

Tableau 8.1. Exemples de séquences d'accès à des ressources et données relatives.

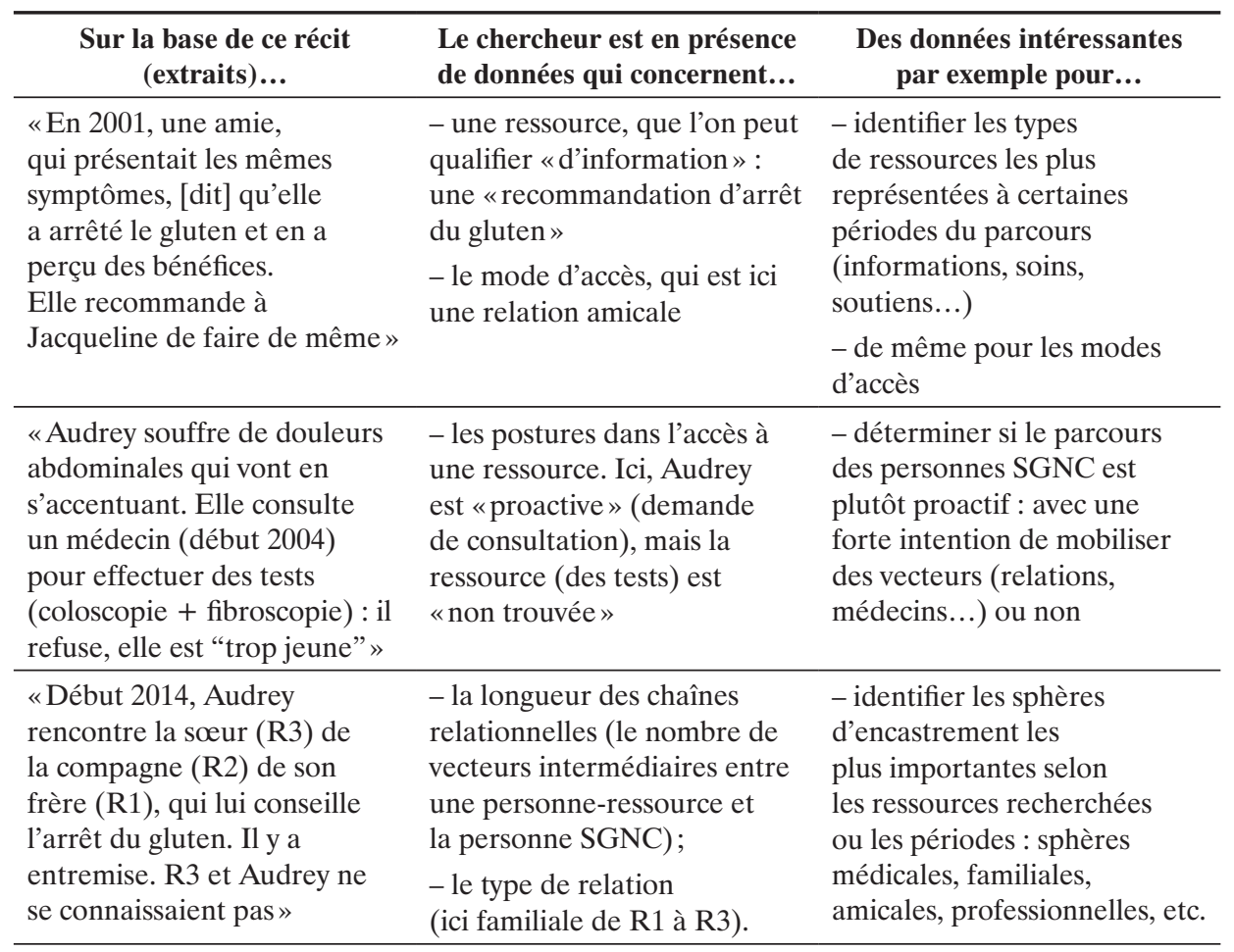

D’une certaine façon, le choix des données à recueillir, à coder et à traiter statistiquement reste dépendant des hypothèses de recherche et cette méthodologie possède une certaine adaptabilité en la matière. Si la question des effets de l'homophilie de genre intéresse le chercheur, il est possible de recueillir et de prendre en compte, pour chacune des séquences, le genre des personnes vectrices de ressources. S'il s'agit d'étudier l'effet de la force du lien sur l'impact de la séquence dans la trajectoire, il est possible de caractériser finement les relations vectrices de ressources (ancienneté, fréquence, type de relation, polyvalence...) afin de tenter d'expliquer pourquoi certaines ressources impactent plus que d'autres.

Une fois les étapes précédentes réalisées, le chercheur peut alors alimenter un tableau (tableau 8.2). Du point de vue de son architecture, chaque ligne correspond à une seule et unique séquence et débute donc par un identifiant. Le reste des informations est renseigné à la suite, dans les colonnes de gauche à droite. Les lignes se suivent de manière chronologique et permettent de détailler, étape après étape, le parcours de la personne et les variables d'intérêt. Une fois l'ensemble de ces séquences répertoriées, la prochaine ligne est celle d'un autre parcours, d'une autre personne. 
Tableau 8.2. Données de contextualisation des séquences.

\begin{tabular}{|c|c|c|c|c|c|c|c|c|}
\hline 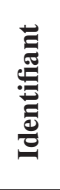 & 总 & 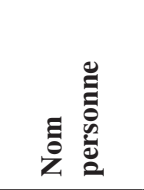 & 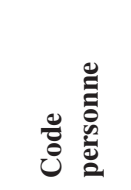 & 总 & 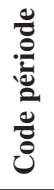 & 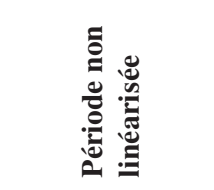 & 递 & 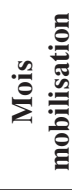 \\
\hline 184 & 1 & Arnaud & RF002 & Antécédents & 1 & Antécédent & 2008 & 1 \\
\hline 185 & 2 & Arnaud & RF002 & Antécédents & 1 & Antécédent & 2009 & 1 \\
\hline 186 & 3 & Arnaud & RF002 & $\begin{array}{c}\text { Errance } \\
\text { de diagnostic }\end{array}$ & 2 & $\begin{array}{c}\text { Errance } \\
\text { de diagnostic }\end{array}$ & 2014 & 1 \\
\hline 188 & 4 & Arnaud & RF002 & Diagnostic & 3 & $\begin{array}{c}\text { Errance } \\
\text { de diagnostic }\end{array}$ & 2014 & 7 \\
\hline 189 & 5 & Arnaud & RF002 & RSG prospère & 5 & $\begin{array}{c}\text { Errance } \\
\text { de diagnostic }\end{array}$ & 2014 & 7 \\
\hline 191 & 6 & Arnaud & RF002 & RSG prospère & 5 & $\begin{array}{c}\text { Errance } \\
\text { de diagnostic }\end{array}$ & 2015 & 2 \\
\hline 192 & 7 & Arnaud & RF002 & RSG prospère & 5 & $\begin{array}{c}\text { Errance } \\
\text { de diagnostic }\end{array}$ & 2015 & 6 \\
\hline 193 & 8 & Arnaud & RF002 & RSG prospère & 5 & $\begin{array}{c}\text { Errance } \\
\text { de diagnostic }\end{array}$ & 2016 & 8 \\
\hline 197 & 9 & Arnaud & RF002 & $\begin{array}{c}\text { Consommation } \\
\text { de blé }\end{array}$ & 6 & $\begin{array}{c}\text { Consommation } \\
\text { de blé }\end{array}$ & 2017 & 4 \\
\hline 198 & 10 & Arnaud & RF002 & $\begin{array}{l}\text { Consommation } \\
\text { de blé }\end{array}$ & 6 & $\begin{array}{c}\text { Consommation } \\
\text { de blé }\end{array}$ & 2017 & 6 \\
\hline 199 & 1 & Lauriane & BDN004 & $\begin{array}{c}\text { Errance } \\
\text { de diagnostic }\end{array}$ & 2 & Antécédents & 2010 & 1 \\
\hline
\end{tabular}

$\mathrm{RSG}=$ régime sans gluten.

Dans les tableaux tableaux 8.3 et 8.4 , le parcours d'Arnaud est détaillé séquence après séquence ${ }^{34}$. On notera qu'une variable peut être précisée au besoin en construisant des sous-catégories : les ressources de type «Informations» comprennent dans notre tableau la sous-catégorie du «conseil d'arrêt du gluten» (distincte du «diagnostic médical») qui peut elle-même être précisée selon que le conseil provient d'une personne exerçant un métier dans le médical ou non. Il s'agit ici encore d'un travail de codage qui est à arbitrer en fonction des traitements envisagés : quelle profondeur voulue ou nécessaire? Ces variables sont renseignées dans le tableau avec des valeurs numériques et accompagnées d'un libellé ${ }^{35}$; un dictionnaire des variables sur une autre feuille peut être utile en cas de collaborations sur le fichier, comme c'est le cas lorsque plusieurs enquêteurs doivent renseigner chacun leurs propres entretiens. Laisser un champ libre destiné aux commentaires ou à l'explicitation de la séquence en fin de ligne (dernière colonne) est aussi un moyen d'assurer une relecture de contrôle rapide d'un récit et de sa transformation en données codées.

34. Dans un souci de lisibilité, seules quelques séquences ont été conservées. Le parcours d'Arnaud compte normalement 18 séquences, soit 18 lignes.

35. Seuls les libellés ont été reproduits dans les tableaux. 
Tableau 8.3. Données de caractérisation des ressources.

\begin{tabular}{|c|c|c|c|c|}
\hline Identifiant & Type de ressource (01) & (02) & Posture & Nom d'accès \\
\hline 184 & Diagnostic médical & Information & Non trouvée & $\begin{array}{l}\text { Son médecin } \\
\text { généraliste (ville) }\end{array}$ \\
\hline 185 & Diagnostic médical & Information & Non trouvée & $\begin{array}{l}\text { Son médecin } \\
\text { généraliste (ville) }\end{array}$ \\
\hline 186 & Informations/médecin & Information & Reçue & $\begin{array}{l}\text { Un médecin } \\
\text { des intolérances } \\
\text { alimentaires }\end{array}$ \\
\hline 188 & Diagnostic médical & Information & Reçue & Laboratoire B. \\
\hline 189 & $\begin{array}{l}\text { Informations/ } \\
\text { variétés anciennes }\end{array}$ & Information & Reçue & $\begin{array}{l}\text { Un médecin } \\
\text { des intolérances } \\
\text { alimentaires }\end{array}$ \\
\hline 191 & Offre marchande & Information & Proactive & Un artisan boulanger \\
\hline 192 & Diagnostic médical & Information & Proactive & Laboratoire B. \\
\hline 193 & Informations /médecin & Information & Proactive & Laboratoire B. \\
\hline 197 & Découverte tolérance & $\begin{array}{l}\text { Savoirs } \\
\text { expérientiels }\end{array}$ & Proactive & Lartisan boulanger \\
\hline 198 & $\begin{array}{l}\text { Informations/ } \\
\text { variétés anciennes }\end{array}$ & Information & Proactive & $\begin{array}{l}\text { Le livre de l'artisan } \\
\text { boulanger }\end{array}$ \\
\hline 199 & $\begin{array}{l}\text { Informations/ } \\
\text { alimentation en général }\end{array}$ & Information & Proactive & Le livre du Dr Delabos \\
\hline
\end{tabular}

Tableau 8.4. Données de caractérisation des accès aux ressources.

\begin{tabular}{|c|c|c|c|c|c|}
\hline Identifiant & $\begin{array}{c}\text { Catégorie } \\
\text { d'accès }\end{array}$ & Type d'accès & Type médiation NR & $\begin{array}{c}\text { Type } \\
\text { relation }\end{array}$ & $\begin{array}{l}\text { Longueur } \\
\text { chaîne }\end{array}$ \\
\hline 184 & Médicale & Médiation NR & Personnel médical & & \\
\hline 185 & Médicale & Médiation NR & Personnel médical & & \\
\hline 186 & Médicale & Médiation NR & Personnel médical & & \\
\hline 188 & Médicale & Médiation NR & Personnel médical & & \\
\hline 189 & Médicale & Médiation NR & Personnel médical & & \\
\hline 191 & Non médicale & Médiation NR & Dispositif marchand & & \\
\hline 192 & Médicale & Médiation NR & Personnel médical & & \\
\hline 193 & Médicale & Médiation NR & Personnel médical & & \\
\hline 197 & Non médicale & Médiation NR & Dispositif marchand & & \\
\hline 198 & Non médicale & Relation & & Amicale & 1 \\
\hline 199 & Médicale & Médiation NR & Livre & & \\
\hline
\end{tabular}

$\mathrm{NR}=$ non relationnelle. 
En procédant ainsi, quelques difficultés peuvent toutefois émerger. Elles proviennent généralement du recueil des données en lui-même, ce qui nécessite de bien définir les informations pertinentes à collecter préalablement à l'étape des entretiens. Elles peuvent aussi résulter de biais de mémoire qui peuvent avoir une incidence sur les capacités des personnes interrogées à préciser certaines années ou mois d'accès à une ressource pour les parcours les plus anciens ou les plus mouvementés; ou de manière plus générale sur la non-évocation d'une séquence, d'une entremise, d'un accès à une ressource, etc. En dehors des éléments susmentionnés, le parcours de certains ne se prête pas vraiment à la codification (teneur exploratoire de l'entretien; parcours très courts avec enchaînements rapides vers l'adoption d'un régime sans gluten...) ou la rend difficile : c'est le cas notamment pour les parcours avec des diagnostics successifs, des périodes de retour à la consommation de céréales contenant du gluten, puis un énième diagnostic, qui imposent des règles de codage. Sur quel critère linéariser le parcours? En ce qui nous concerne, nous avons considéré que les séquences qui créaient un précédent (un changement d'une période à une autre) inscrivaient le reste des séquences dans cette période jusqu'à une prochaine séquence «charnière». Pour le dire autrement, une confirmation diagnostique (un gluten challenge ${ }^{36}$ par exemple) au cours d'une période de «régime sans gluten prospère ${ }^{37}$ ne conduisait pas à comptabiliser cette ressource comme relevant de la période du «diagnostic» si cette étape avait déjà été franchie auparavant. Au vu de ces éléments, notre terrain nous a permis de coder le parcours de 31 personnes sur 38 rencontrées, avec une moyenne de séquences par personne de 11 (minimum $=4$; maximum $=25$ ) pour un tableau comprenant 346 séquences (en ligne) et 32 variables (en colonne).

Le fichier peut ensuite s'exporter dans un logiciel statistique et des traitements peuvent être menés. Dans notre cas, à l'aide de SPSS (Statistical Package for the Social Sciences), nous avons entre autres été en mesure de :

- savoir combien de séquences d'accès à des informations ont fait l'objet d'une posture proactive ou passive tout au long des parcours. On sait ainsi que les ressources obtenues durant les parcours sont majoritairement recherchées de manière active par les personnes : $80 \%$ des séquences relèvent de cette posture ( $20 \%$ des ressources sont obtenues de manière passive);

- savoir quels sont les types de ressources acquises pendant les trajectoires en profitant du découpage en périodes pour identifier celles qui sont majoritairement obtenues au moment de la transition vers une alimentation sans gluten par exemple. En ce qui concerne cette période de changements alimentaires, ce sont les sites Internet

36. Réintroduction médicalement assistée du gluten comme mesure de confirmation du diagnostic de la SGNC: en raison de l'absence de biomarqueurs, le gluten challenge permet d'observer la réapparition des symptômes et de statuer sur la sensibilité. Dans les faits, cette pratique est très rare $(n=2 / 38)$ et il s'agit le plus souvent d'expériences personnelles de réintroduction intentionnelles ou accidentelles (Akermann et Coeurquetin, à paraître).

37. Le «régime sans gluten prospère» se définit comme la période durant laquelle la personne entreprend et parvient à supprimer complètement les aliments sources de gluten de son alimentation quotidienne. Ce terme s'oppose au «régime sans gluten intermittent» qui consiste pour la personne SGNC à limiter sa consommation de produits contenant du gluten sans qu'une éviction stricte ne soit envisagée. Cette période met au jour les nombreuses difficultés liées à la perspective, à la mise en place et au maintien d'un régime restrictif au long cours. Le régime sans gluten intermittent peut-être un préalable à l'adoption d'un régime prospère. 
et autres médias qui deviennent les vecteurs non relationnels les plus importants pour soutenir les débuts parfois difficiles des personnes SGNC dans leur éviction : $80 \%$ de l'usage des médias se situe dans cette période et permettent d'obtenir des idées de recettes, des informations sur les produits alimentaires adaptés, du soutien, etc.; - savoir quels types de médiations sont les plus pourvoyeurs de ressources selon les étapes afin de quantifier l'importance des différents «mondes» (médical, marchand, numérique...) et des cercles sociaux (familial, amical, professionnel...) dans le cas de la SGNC, de son diagnostic et des changements alimentaires qu'elle occasionne. De manière générale, alors que le monde médical est surreprésenté au moment de l'errance diagnostique et du diagnostic, la période de mise en place d'un régime sans gluten ou du basculement vers les produits céréaliers artisanaux sollicitent beaucoup plus les relations familiales, amicales, les médias et les espaces marchands;

- savoir quand interviennent les professionnels de santé, combien d'entre eux sont en moyenne consultés avant le diagnostic et si ces derniers appartiennent plutôt au monde médical conventionnel ou alternatif. On peut dire à ce sujet que $25 \%$ des personnes interrogées ont rencontré au minimum un professionnel de santé durant la période de l'errance diagnostique : la moyenne se situant à 2,7 praticiens, toute orientation confondue (maximum $=6$ ). Les ressources sont en grande majorité des «informations » et proviennent, pour $55 \%$ d'entre elles, de praticiens de médecine conventionnelle et $24 \%$ de médecines alternatives ${ }^{38}$.

En somme, les traitements statistiques permettent donc de mettre en lumière et généralisent les processus de changements alimentaires chez les personnes SGNC, les ressources qui équipent les mangeurs dans leurs démarches, en considérant aussi la place des prescripteurs (Golley et al., 2015). Après une partie parfois longue de préparation des données (production d'un récit, codage, construction d'une base de données), des résultats sont très vite obtenus à l'aide de statistiques courantes (fréquences et tableaux croisés). Pour compléter ces résultats, les entretiens sont aussi des sources précieuses d'information, qui peuvent être traitées à l'aide d'une analyse thématique. Pour ce qui est de ce projet, ces analyses ont porté sur les postures à l'égard des institutions médicales; sur les perceptions corporelles comme une ressource diagnostique dans un contexte d'incertitude clinique; ou encore sur les comportements et pratiques à l'occasion des prises alimentaires commensales (stratégies d'évitement et de compensation), etc. L'analyse qualitative a aussi permis de mieux comprendre ce qui se joue lorsque les personnes SGNC deviennent prescriptrices à leur tour. La méthode des narrations quantifiées peut alors se trouver enrichie si besoin d'une approche bidirectionnelle en envisageant aussi la personne SGNC comme émettrice de ressources. Un premier objectif pourrait être celui de porter son attention sur les ressources allant des autres vers soi, pour ensuite aborder ce basculement du statut de la personne SGNC : de récepteur à celui d'émetteur. Enfin, de manière générale, les deux approches se révèlent complémentaires : certaines analyses statistiques peuvent être illustrées et mieux comprises à l'aide des analyses qualitatives compréhensives et ces dernières peuvent être généralisées à l'aide des premières.

38. Dans le cadre de cette recherche, les «praticiens alternatifs» regroupent les professionnels de santé qui ne sont pas docteurs en médecine (ostéopathes, acupuncteurs, naturopathes, énergéticiens...) et les professionnels diplômés de médecine exerçant des approches non conventionnelles (homéopathes, kinéostéopathe utilisant la méthode Poyet par exemple). 


\section{W De la méthode au contexte d'utilisation : ajuster les relances au terrain étudié}

Au regard de notre propre utilisation des «narrations quantifiées » dans le cadre du projet «Gluten, mythe ou réalité ?», cette méthode ne semble pas nécessiter une adaptation aux publics enquêtés plus importante que celle qui peut être demandée lors des enquêtes de terrain par entretiens sociologiques semi-directifs à trame biographique : la rencontre et l'échange entre l'enquêteur et l'enquêté restent en substance les mêmes. Il faut peut-être noter la propension de certains enquêtés (souvent les plus diplômés) à orienter le récit sur leurs opinions ou leurs motivations plutôt que sur des éléments très factuels et qui leur paraissent parfois anodins, voire inutiles, tels que les dates, les types de relations, le nom d'un site Internet ou d'un magasin. L'enquêteur doit alors constamment rediriger l'entretien sur l'action, et réussir à obtenir toutes les informations nécessaires au codage du récit au moyen de relances précises. Pour autant, après quelques minutes d'entretiens, les enquêtés comprennent l'intérêt systématique du chercheur pour «les détails » et ont tendance à apporter par eux-mêmes les précisions attendues.

La principale adaptation de la méthode au contexte vient de la nécessité d'ajuster les relances aux terrains étudiés. Sur certains terrains, la majorité des trajectoires sont ponctuées de séquences similaires ou de phases de recherche de certains types de ressources spécifiques qu'il convient de découvrir au moyen d'entretiens exploratoires, afin de pouvoir procéder, dans la phase d'entretiens semi-directifs, à des relances systématiques. Par exemple, dans le cas de la consommation sans gluten, il peut être utile de savoir que certaines personnes recherchent du soutien moral auprès de leur entourage proche pour systématiser des relances visant à découvrir la présence ou l'absence de ce type de ressource dans les trajectoires des personnes. Dans le cas d'étude de création d'association (Amap, groupement d'achats...), certaines étapes sont inévitables, comme la rédaction des statuts. Le chercheur doit donc avoir en tête ces «points de passage obligés » afin d'encourager l'enquêté à les aborder au cours de l'entretien, lorsqu'il ne le fait pas de lui-même. On rejoint ici les problématiques liées à la conduite d'entretiens semidirectifs qui doivent trouver leur équilibre entre la systématisation des relances et les phases de non-directivité.

La partie analytique quant à elle n'est pas concernée par ces besoins d'adaptation, si ce n'est qu'il paraît nécessaire d'ajuster les catégories des variables selon les cas rencontrés : suffisamment spécifiques pour accueillir l'hétérogénéité des parcours de vie et suffisamment génériques pour obtenir des résultats généralisables.

On notera également que cette méthode nécessite des entretiens approfondis et, selon les objets de recherche, de faire se remémorer des événements parfois désagréables ou intimes aux personnes rencontrées. Dans le cas des femmes et des hommes se déclarant sensibles au gluten non coliaques, les parcours parfois très médicalisés, la proactivité de leurs démarches, puis la résolution de leurs problèmes, la thématisation scientifique de ce désordre longtemps déconsidéré et la conduite de certains entretiens par téléphone ont contribué à ce que les enquêtés échangent avec nous sans difficulté notable. 
La méthode des narrations quantifiées est une méthode mixte d'analyse, qui permet après quelques adaptations d'analyser finement les trajectoires individuelles ou collectives de mangeurs qui entreprennent des changements actifs dans leurs pratiques alimentaires. Elle est susceptible d'intéresser les chercheurs qui souhaitent comprendre les processus de bifurcation alimentaire en se décentrant des rationalités des personnes pour donner plus de place aux contextes d'actions. Comprendre le rôle des relations, des dispositifs, et plus largement des appuis sur lesquels repose la mise en œuvre des pratiques alimentaires constitue une piste de développement pour les chercheurs qui souhaitent analyser la manière dont les pratiques sont adoptées et se diffusent dans certains contextes sociaux et territoriaux.

\section{"Références}

Akermann G., Barthe J.-F., Defossez A., 2018. Dynamique des réseaux personnels à l'épreuve des maladies graves et de longue durée. Temporalités, 7. https://doi.org/10.4000/temporalites.4016

Akermann G., Coeurquetin P., à paraître. Consommer sans gluten : trajectoires individuelles d'éviction, in Goulet F., Vinck D. (éd.), Faire sans, faire avec moins : les nouveaux horizons de l'innovation, Paris, Presses des Mines.

Bertaux D., 1997. Les récits de vie : perspective ethnosociologique, Paris, Nathan (Collection 128, Sociologie), $128 \mathrm{p}$.

Chauvin P.-M., Grossetti M., Zalio P.-P. (éd.), 2014. Dictionnaire sociologique de l'entrepreneuriat, Paris, Les Presses de Sciences Po (Collection Domaine Gouvernances), 640 p.

Degenne A., Forsé M., 1994. Les réseaux sociaux : une analyse structurale en sociologie, Paris, Armand Colin (Collection U, série Sociologie), 263 p.

Golley S., Corsini N., Topping D., Morell M., Mohr P., 2015. Motivations for avoiding wheat consumption in Australia: results from a population survey. Public Health Nutrition, 18 (3), 490-499. https://doi.org/10.1017/s1368980014000652

Granovetter M., 1985. Economic Action and Social Structure: The Problem of Embeddedness. Amercian Journal of Sociology, 91 (3), 481-510. https://doi.org/10.1086/228311.

Grossetti M., Barthe J.-F., 2008. Dynamique des réseaux interpersonnels et des organisations dans les entreprises. Revue française de sociologie, 49 (3), 582-612. https://doi.org/10.3917/rfs.493.0585

Grossetti M., 2011. Les narrations quantifiées : une méthode mixte pour étudier des processus sociaux. Terrain \& Travaux, 19 (2), 161-182. https://doi.org/10.3917/tt.019.0161

Grossetti M., Barthe J.-F., Chauvac N., 2011. Studying Relational Chains from Narrative Material. Bulletin de méthodologie sociologique, 110 (1), 11-25. https://doi.org/10.1177/0759106311399553 


\title{
Chapitre 9 \\ L'(auto-)biographie : une démarche compréhensive pour accéder à la subjectivité des mangeurs
}

\author{
AMANDINE RoCHEDY ${ }^{a}$ ET TRISTAN FOURNIER ${ }^{b}$ \\ ${ }^{a}$ Université de Toulouse Jean Jaurès - ISTHIA, CERTOP - CNRS, France. \\ ${ }^{\mathrm{b}}$ CNRS - IRIS, EHESS, Paris, France.
}

\begin{abstract}
Cette méthode qualitative s'appuie sur un matériau d'enquête intégralement produit par les enquêtés eux-mêmes, qu'il s'agisse de l'étude de documents personnels de seconde main (biographie) ou d'un travail d'écriture sur soi commandité par l'enquêteur (autobiographie). Dans les deux cas, l'objectif consiste à appréhender ces écrits comme des produits sociaux. Le but n'est pas tant de viser l'exactitude des faits que de reconstruire l'expérience alimentaire telle qu'elle a été vécue et qu'elle est racontée.
\end{abstract}

«Je situe l'origine de mon intérêt pour les documents personnels dans une longue lettre ramassée un jour de pluie dans une allée derrière ma maison : une lettre écrite par une jeune fille qui suivait un enseignement à l'hôpital, adressée à son père et concernant les relations et disputes dans la famille. Je ressentis alors que l'on apprendrait beaucoup si l'on disposait d'un nombre important de lettres de cette sorte». C'est souvent par cette anecdote, relatée par William Isaac Thomas dans Le paysan polonais (Thomas et Znaniecki, 1998), que l'on situe les premiers intérêts sociologiques pour le travail (auto-)biographique. Nous sommes au début du $\mathrm{xx}^{\mathrm{e}}$ siècle et la sociologie a encore besoin de faire ses preuves en tant que discipline scientifique. Elle est alors dominée par la tradition durkheimienne, qui implique un travail d'objectivation des faits sociaux : l'enquêteur est au premier plan et son rôle, qu'il s'agisse d'élaboration d'hypothèses, d'analyses statistiques ou de relances durant les entretiens, est central. Thomas, qui deviendra l'une des figures de l'école de Chicago, propose au contraire de partir de la subjectivité des individus et d'en constituer une grille de lecture du social. Ayant obtenu des crédits d'une fondation privée pour entreprendre une enquête sur les modes de vie des communautés d'immigrés à Chicago - la démographie de la ville a littéralement explosé en quelques décennies -, il va travailler à partir d'un corpus composé de lettres, de témoignages et de biographies, écrits par des migrants polonais. Malgré le fait qu'il s'agisse de documents de seconde main, il démontrera l'intérêt de cette méthode en 
arguant le fait qu'il n'aurait probablement jamais accédé à un matériau aussi riche par des méthodes plus classiques. De cette vaste enquête sera tiré «le théorème de Thomas », sorte de prophétie autoréalisatrice qui rend compte du fait que les comportements des individus s'expliquent par leur perception de la réalité et non par la réalité elle-même : «si les hommes définissent des situations comme réelles, alors elles sont réelles dans leurs conséquences».

\section{W Institutionnalisation de la méthode}

Cette tradition de recherche sur les récits de vie va s'étoffer tout au long du $\mathrm{xx}^{\mathrm{e}}$ siècle, puis s'institutionnaliser, dans la littérature anglo-saxonne, sous le terme «auto/biography». La distinction entre «auto» et «biography» provient du fait que la méthode ambitionne d'embrasser deux outils distincts, mais similaires d'un point de vue épistémologique, le matériau étant intégralement produit par l'enquêté. La biographie renvoie à l'étude de documents personnels de seconde main que le sociologue a identifiés comme potentiellement porteurs de sens (pensons aux lettres des migrants polonais étudiées par Thomas et Znaniecki); l'autobiographie implique qu'un travail d'écriture sur soi ait été commandité au préalable par le sociologue, et ce, qu'il soit écrit par l'enquêté ou par le sociologue lui-même (il était par exemple demandé aux futurs étudiants du département de sociologie de l'école de Chicago de rédiger leur propre autobiographie de manière à aiguiser leur réflexivité sur le monde social). Dans les deux cas, l'objectif consiste à appréhender ces écrits comme des produits sociaux. D'un point de vue institutionnel, c'est à la suite d'une conférence organisée en 1992 à l'université de Manchester et intitulée «Sociology, biography and autobiography» qu'un groupe de recherche «Auto/Biography» sera officiellement créé à la British Sociological Association. L'année suivante, un numéro rassemblant dix contributions sur l'(auto-)biographie comme méthode d'enquête sera publié dans la très légitimante revue Sociology.

En France, la méthode va être mobilisée en sciences sociales à partir des années 19701980. Elle est alors adossée aux travaux de l'école de Chicago et s'inspire également de ceux menés dans d'autres disciplines, notamment l'histoire et la littérature. En sociologie, deux voies d'institutionnalisation de la méthode peuvent être repérées. La première est la publication en 1986 d'un numéro spécial de la revue Actes de la recherche en sciences sociales dédié à la méthode biographique et dirigé par Pierre Bourdieu. Ce numéro rassemble treize publications, dont certaines émanent de chercheurs renommés comme Howard S. Becker. L'audience est importante, d'autant que le numéro alimentera un vif débat (nous l'abordons plus loin dans la section «Intérêts et limites»). La seconde voie d'institutionnalisation est la publication, quelques années plus tard, de deux manuels méthodologiques. D’abord La méthode biographique de Jean Peneff (1990), qui s'appuie à la fois sur les travaux réalisés par les chercheurs de l'école de Chicago, que l'auteur a rencontrés et longuement interrogés lors de séjours aux États-Unis, et sur des enquêtes de terrain qu'il a lui-même réalisées auprès d'industriels algériens, d'instituteurs ruraux et de militants syndicalistes ouvriers. Pour Peneff, l'objectif n'est pas tant de reconstruire la mémoire des enquêtés que de recueillir des informations précises sur leur environnement social. Il privilégie les autobiographies, c’est-à-dire les « récits travaillés et construits 
selon un schéma préétabli, d'une longueur conséquente avec des précisions et une chronologie suivie, récits obtenus à la suite d'un effort de recherche entrepris avec l'aide d'un sociologue ou l'initiative du narrateur, qui entend faire un document démonstratif» (Peneff, 1990, p. 102), aux histoires de vie qu'il considère comme des entretiens libres sans contrôle. Plus tard, en 1997, Daniel Bertaux publie Le récit de vie, un manuel classique bien connu des étudiants en sociologie, qui va contribuer à légitimer l'entrée par les discours des enquêtés, mais, dans le même temps, diluer la méthode (auto-)biographique dans ce qu'il nomme plus largement les récits de vie. C'est probablement pour cette raison que le terme même d'(auto-)biographie est bien moins usité dans les sciences sociales françaises qu'anglo-saxonnes.

Enfin, il est à noter un regain d'intérêt et de recours à la méthode (auto-)biographique depuis quelques années, et ce pour deux raisons concordantes. D'une part, se développent des espaces où les individus peuvent, au quotidien, se raconter facilement et librement (les réseaux sociaux en étant la parfaite incarnation). Ce tournant va favoriser l'émergence de la «nethnographie», une méthode d'enquête qui analyse les actes communicationnels des membres d'une communauté virtuelle. D'autre part, la montée en charge des formes de réflexivité (notamment alimentaire) et des processus d'individuation dans les sociétés contemporaines renforce considérablement l'entreprise (auto-)biographique : être attentif à soi et se raconter deviennent des actes banals, qui permettent la formulation d' «égo-récits» via des pages Internet, des forums de discussion, des séances chez le psychologue ou avec un coach. Comme stipulé dans le récent ouvrage Parler de soi du Collectif B. (2020), «l'approche biographique est au cœur du renouvellement des sciences sociales».

\section{W Intérêts et limites}

Les promoteurs de la méthode (auto-)biographique arguent du fait qu'il s'agit d'une démarche compréhensive pertinente pour accéder à la subjectivité des individus et ainsi saisir les stéréotypes en cours et le poids des normes sociales sur les discours. Effectivement, l'objectif n'est pas tant de viser l'exactitude des faits que de reconstruire la réalité telle que les enquêtés la perçoivent ou la relatent : leur réalité. Dans le cas de la biographie, les enquêtés ne savent a priori pas qu'ils participent à une enquête de sciences sociales (les immigrés polonais de Chicago n'imaginaient pas, en les écrivant, que leurs lettres personnelles seraient plus tard analysées par des sociologues). Le matériau collecté est de seconde main et l'enjeu pour l'enquêteur consiste à resituer le contexte sociohistorique dans lequel il a été produit. Dans le cas de l'autobiographie, l'enquêté sait qu'il est sollicité pour une enquête de sciences sociales. Le matériau est de première main, la demande de l'enquêteur peut alors être guidée ou quelque peu orientée (par exemple : "pourriez-vous raconter, par écrit, la relation que vous avez avec l'alimentation depuis votre enfance?»). Toutefois, l'objectif reste d'influencer le moins possible le «travail» de l'enquêté. C'est lui qui produit véritablement les données de l'enquête et ceci revêt deux avantages considérables. Le premier est d'ordre épistémologique : une très grande liberté lui est accordée, qu'il s'agisse de contenu (l'enquêteur n'est pas présent pour cadrer, contrôler ou relancer le propos), de temporalité (le travail d'écriture peut être réalisé sur une période plus ou moins longue, il peut être interrompu, puis 
repris) ou d'influences (contrairement à une situation plus classique d'entretien, ici l'enquêteur est absent et l'enquêté peut alors solliciter, au besoin, des personnes de son entourage susceptibles de l'aider à se remémorer les événements du récit). Le rôle de l'enquêteur est volontairement minimisé et la posture est dite non hiérarchique. En effet, les promoteurs de la méthode (auto-)biographique insistent sur le fait que l'enquêteur peut perturber la mise en récit, notamment lorsqu'il ne partage pas les mêmes attributs sociaux que l'enquêté (en termes de classe, de race, de genre). Le biais de désirabilité sociale - le fait de produire un discours que l'on pense être celui attendu par l'enquêteur - est le principal risque d'une relation asymétrique. L'objectif est donc de laisser carte blanche à l'enquêté, avec une perspective émancipatoire revendiquée, notamment d'un point de vue féministe : écrire sur soi et reconstruire sa trajectoire de vie peut permettre de développer une réflexivité critique quant à sa propre condition sociale et inciter le dialogue avec d'autres membres de sa communauté. Le second avantage de la méthode (auto-) biographique est d'ordre plus pragmatique : elle permet à la fois un gain de temps (puisque ce sont les enquêtés qui produisent le matériau, une partie de l'enquête se fait en quelque sorte d'elle-même) et de moyens (comme l'enquête ne se fait pas de visu, il n'est pas nécessaire à l'enquêteur de se déplacer, les interactions pouvant alors se réaliser à distance par courrier électronique).

En revanche, la méthode a également suscité des critiques, la première étant liée à l'exercice lui-même : écrire, et qui plus est se raconter par l'écriture, est une pratique qui requiert du temps libre et des compétences. La consigne même peut donc exercer une certaine violence symbolique lorsqu'elle est proposée à des personnes qui ne sont pas coutumières de ce mode d'expression et qui pourraient avoir le sentiment de ne pas savoir exprimer clairement leurs idées, de ne pas pouvoir écrire sans faire de fautes d'orthographe ou de syntaxe, bref d'être jugées négativement. Ce possible biais de sélection sociale doit être considéré.

Plus largement, la méthode a fait l'objet d'importants débats en sciences sociales. En 1986, Pierre Bourdieu coordonne un numéro intitulé «L'illusion biographique » dans Actes de la recherche en sciences sociales, revue qu'il dirige alors. Il y développe une critique en deux principaux points. D'abord à l'égard du matériau collecté dans le cadre d'enquêtes (auto-)biographiques, matériau qui se prêterait selon lui à une «sociologie du soupçon»: comment ne pas douter de la véracité des propos tenus par les enquêtés? En quoi ne seraient-ils pas le fruit de mensonges ou, du moins, le produit d'une déformation ou d'une dissimulation plus ou moins consciente de la réalité? Entre omission et sélectivité, il pointe la complexité de la relation entre vie vécue et vie écrite. Il attire ainsi l'attention sur l'illusion d'une identité reconstruite, d'un lissage ou d'une homogénéisation de parcours, d'une volonté de donner du sens a posteriori à des événements qui n'en avaient pas nécessairement au moment vécu. C'est ensuite la méthode en elle-même qui lui pose problème, notamment le fait que ce type d'enquête se fasse quasiment en l'absence d'enquêteur. Comme il s'agit d'un exercice narratif, c'est la perception individuelle des événements qui prime. Or l'intérêt même de l'enquête de sciences sociales réside, selon lui, dans la mise en perspective de ce point de vue individuel avec un point de vue plus collectif où s'expriment les structures sociales. Autrement dit, l'enquêté si sincère soit-il, ne saurait se substituer à l'enquêteur, dont le métier est justement d'articuler les niveaux 
micro et macro, de resituer les propos et logiques exprimés par un individu dans des mécanismes sociaux plus larges, le plus souvent inaccessibles à l'enquêté. Ces critiques alimenteront un vif débat, y compris au sein même de ce numéro d'Actes de la recherche en sciences sociales, car certains des chercheurs sollicités par Bourdieu - Mickael Pollack en tête - travaillaient à partir de cette méthode et n'avaient alors pas imaginé contribuer à une réflexion critique sur le sujet. Mais, comme l'a souligné bien plus récemment Nathalie Heinich (2010), à ce propos, Bourdieu s'est peut-être enferré dans «l'illusion d'une illusion » pour des raisons autres qu'épistémologiques. Elle relate ainsi sa prise en marche tardive du train biographique - d'autres travaillaient déjà à partir de cette méthode depuis longtemps - ce qui pourrait expliquer cette critique exacerbée en vue de «marquer le territoire». Elle insiste aussi sur la méfiance de Bourdieu à l'égard de la «cure analytique», lui qui œuvrait pour la sociologie et souhaitait donc se démarquer de toute entreprise psychanalytique. Il mobilisera toutefois ladite méthode quelques années plus tard dans La misère $d u$ monde. À l'évidence, la méthode (auto-)biographique a opposé et oppose toujours les tenants d'une sociologie déterministe, surtout attentive au poids des structures sociales, à ceux d'une sociologie qui s'intéresse plutôt aux capacités d'agir et de réflexivité des individus.

\section{- Application au domaine alimentaire}

Si de plus en plus d'enquêtes de sciences sociales intègrent l'approche (auto-)biographique, cette dernière pose des questions particulières à celles et ceux intéressés par l'étude de l'alimentation.

D'abord, le fait que la méthode s'adresse explicitement à des personnes ayant accepté d'écrire à propos d'elles et de leur alimentation n'est pas anodin. Cet aspect induit une sélection sociale de la population d'enquête. La réflexivité alimentaire est effectivement socialement située : même si les plus vulnérables ne sont pas prêts à manger n'importe quoi, on sait par de nombreuses enquêtes que c'est en haut de l'échelle sociale que l'on considère le plus l'impact potentiel de l'alimentation sur l'état de santé, le corps ou l'environnement. L'alimentation est une problématique du long terme, qui enferme les plus démunis dans l'urgence du quotidien et invite les plus aisés à se projeter dans le futur. Faire appel à cette réflexivité conduit donc à s'adresser, même implicitement, à des personnes dotées de capitaux socioculturels importants. Il convient donc, pour les chercheurs intéressés par cette méthode, de considérer ce possible biais de sélection sociale ${ }^{39}$, tout comme les aspects éthiques de son utilisation.

Ensuite, l'(auto-)biographie se révèle être à rebours d'une tradition d'enquêtes dont l'objectif est de reconstruire le plus fidèlement possible les pratiques alimentaires pour ainsi approcher au plus près la réalité des comportements. Car, à moins de confier une caméra ou un appareil photo aux enquêtés ou alors de poster un

39. Ce biais est surtout à considérer pour des enquêtes autobiographiques réalisées en population générale. Rappelons toutefois que la méthode biographique a été développée à Chicago afin de saisir les subjectivités de paysans et d'ouvriers immigrés, et ainsi contrebalancer les rapports officiels qui décrivaient trop grossièrement les modes de vie de ces populations discriminées. 
enquêteur chez eux - protocole critiquable scientifiquement et condamnable éthiquement, sujet magnifiquement traité dans le savoureux film Kitchen stories sorti en 2003 -, les sociologues doivent le plus souvent se contenter de reconstruire les pratiques alimentaires de leurs enquêtés, par oral et/ou par écrit. Le rappel des 24 heures, un outil classique des sciences de la nutrition, qui a depuis été importé et actualisé dans le domaine des sciences sociales (Bossard et al., 2010), se révèle assez efficace pour objectiver les pratiques alimentaires des individus. De surcroît, il permet aux sociologues d'établir un dialogue avec d'autres disciplines - nutrition et épidémiologie notamment -, qui travaillent à une échelle où l'exactitude des consommations alimentaires est cruciale. La méthode (auto-)biographique considère, elle, que le recueil des normes alimentaires est tout aussi important que celui des pratiques réelles : elle vise justement à prendre au sérieux les propos des enquêtés car, même distants de la réalité, ils s'avèrent être particulièrement porteurs de sens. En cela, elle joue moins le jeu de l'interdisciplinarité, mais permet en revanche d'aller plus loin dans l'analyse sociologique des processus de socialisation, de moralisation des conduites et de performativité des discours, des modes, des normes. Pour ce faire, elle peut être utilisée en complément d'autres méthodes (observations, entretiens, questionnaires, etc.) ou avec le support d'outils (photo ou vidéo notamment), mais aussi de manière plus autonome et indépendante, comme le démontrent les deux exemples d'enquête qui suivent.

\section{W Deux exemples d'autobiographies alimentaires}

\section{Les intermittents du bio}

Dans son enquête, Claire Lamine (2008) s’intéresse aux «mangeurs bio intermittents », définis comme des individus qui achètent à fréquence irrégulière et de façon variable des produits biologiques. Plus largement, elle appréhende - dans une perspective compréhensive - la pluralité et la variabilité des pratiques et des choix alimentaires dans la société contemporaine et ce, en lien avec les crises alimentaires. L'auteure utilise l'approche des trajectoires alimentaires afin d'identifier pourquoi et comment les mangeurs adoptent le «bio» dans certaines de leurs pratiques. Autrement dit, l'étude identifie ce qui fait continuité ou rupture dans ces trajectoires, avec une attention accordée aux déclencheurs de changement. Dans la démarche d'enquête, les autobiographies alimentaires ont été recueillies auprès des enquêtés (15 autobiographies alimentaires obtenues auprès des 22 mangeurs interrogés) afin qu'ils «se livrent à la narration libre de leurs histoires de vie dans leur lien à la nourriture» (Lamine, 2008, p. 30). La consigne de départ était brève et aucun découpage temporel n'était fait. Seules quelques thématiques, comme les souvenirs d'enfance ou les préférences actuelles, étaient mentionnées. Après quelques essais, l'auteure ne faisait plus mention du thème des risques alimentaires dans la consigne de départ afin de ne pas influencer les enquêtés sur cette thématique et au contraire les laisser libres d'en parler ou non. Cette méthode «restitue la façon dont les mangeurs retracent eux-mêmes leurs parcours et reconstruisent leur structure narrative et leurs catégorisations, avec davantage d'autonomie par rapport aux questions du chercheur» (Lamine, 2008, p. 60). Même si, dans les textes collectés, les enquêtés 
mettent en exergue plus facilement les pratiques valorisées que les pratiques réelles, des outils complémentaires vont être utilisés après l'autobiographie alimentaire : entretiens compréhensifs ${ }^{40}$ et, pour certains d'entre eux, observations de séquences d'achat, de préparation et de consommation de repas.

Lamine souligne la diversité des styles et des contenus des autobiographies alimentaires. À travers la liberté de catégorisation, les autobiographies alimentaires mettent en exergue les rapports à la nourriture. Le rapport sensoriel est particulièrement raconté, «vraisemblablement parce que le corps à corps alimentaire est plus facilement écrit dans une forme proche du journal intime que livré dans une discussion de vive voix à un interlocuteur qui éventuellement juge » (Lamine, 2008, p. 69). Au-delà, les textes révèlent les rapports pragmatiques (avoir prise ou non sur son alimentation) et les rapports sociaux et familiaux (opposition entre individuel et collectif) à l'alimentation. Même si les individus ne s'expriment pas à travers une chronologie, comme il est d'usage dans le récit de vie, ils donnent à voir des étapes importantes dans leur cycle de vie et racontent un ou des épisode(s) de leur expérience vécue, qui s'entrecroise(nt) ou s'ajoute(nt) aux autres. L'analyse de ce que les mangeurs racontent eux-mêmes à propos de leurs choix permet ainsi d'identifier des catégories de trajectoires d'adoption des produits «bio» en rendant «visibles les opérations perceptuelles, cognitives et axiologiques autrement repliées dans des routines et des doctrines» (Lamine, 2008, p. 10), comme le souligne Francis Chateauraynaud dans la préface de l'ouvrage.

\section{Gender, class and food}

À partir des récits alimentaires autobiographiques de 75 hommes et femmes, Parsons (2015) analyse les pratiques et représentations alimentaires britanniques contemporaines. Pour tenter de comprendre comment le genre et la classe se nouent autour de l'alimentation, l'auteure a mobilisé une approche constructiviste dite «grounded theory». L'accent est davantage mis sur la narration que sur les pratiques alimentaires réelles. Ce qui intéresse Julie M. Parsons, c'est la façon dont les individus racontent leur histoire et leurs souvenirs alimentaires et comment ils les rendent publics. L'étude consiste donc en une analyse des normes sociales à l'intersection de l'alimentation, du genre et de la classe. Concrètement, elle a invité des participants potentiels, identifiés sur Internet ou via son réseau social élargi, à écrire leur autobiographie alimentaire, puis à lui renvoyer à une adresse électronique créée pour l'occasion ${ }^{41}$. La consigne était volontairement vague pour laisser le plus de liberté possible aux enquêtés, y compris quant aux modalités d'écriture et de rendu (on écrit ce que l'on veut, comme on veut, quand on veut, avec qui l'on veut). Le choix de cette méthode - contact virtuel, liberté d'écriture, entretien asynchronique - est justifié par l'auteure en ce qu'elle représenterait une approche

40. S'inspirant de l'anthropologie, l'entretien compréhensif consiste à considérer les interviewés comme des informateurs de manière à saisir leur réalité et ainsi comprendre les processus sociaux. Pour cela, la conduite de l'entretien doit s'approcher du cadre d'une conversation qui mêle écoute, empathie, relances, prise de distance et analyse critique. L'enquêteur est équipé d'un guide d'entretien qui ne consiste pas en une série de questions précises, ce qui l'enfermerait dans son raisonnement, mais en des thèmes de discussion à aborder et qui sont reliés à des hypothèses de recherche.

41. ourfoodstories@email.com 
émancipatrice et non hiérarchique de la recherche qui permettrait aux répondants d'y participer selon leurs propres termes et modalités. Une fois les textes recueillis, une relance était parfois effectuée par email pour préciser certains éléments. Une analyse thématique classique était ensuite réalisée. Parsons précise qu'à ce stade, c'est la temporalité qui représente le principal enjeu : reconstruire des souvenirs d'enfants avec des yeux d'adultes. Les souvenirs sont en effet souvent racontés sous le mode de l'extraordinaire. De surcroît, ce sont surtout les événements perçus comme extraordinaires qui sont relatés dans un travail autobiographique. Elle donne ainsi l'exemple d'un homme de 51 ans qui décrit avec engouement et précision les «énormes petits-déjeuners du dimanche préparés par son père avec du bacon, des œufs, des tomates, des champignons et du pain grillé, pris sur la terrasse en été avec vue sur le jardin $»^{42}$ (Parsons, 2015, p. 29). Que ce souvenir ait été idéalisé ou non, là n'est pas la question : ce qui intéresse l'auteure est de repérer les formes de socialisation alimentaire et les stéréotypes, notamment de genre et de classe, qui y sont associés. Ici, les souvenirs que cet homme relate à propos des expériences culinaires de son père sont de l'ordre de l'épicurisme, du spectaculaire et de l'extraordinaire. Il en va à l'inverse des repas du quotidien préparés par les mères, soit qu'ils brillent par leur absence au sein des récits, soit qu'ils soient associés à des discussions alors vécues comme ennuyeuses à propos de nutrition ou de manières de table. Les récits donnent ainsi à voir une division du travail genrée dans la sphère domestique : la nourriture comme travail pour les femmes avec des préoccupations de care, la nourriture comme plaisir pour les hommes avec des dimensions ludiques et récréatives.

\section{" Un exemple de biographie alimentaire}

Écrire la vie, celle des autres ou la sienne, n'est pas nouveau et s'avère même de plus en plus répandu, y compris dans le cas de l'autisme. Les années 1990, avec l'élargissement des troubles autistiques et la visibilité des personnes autistes sans déficience, voient une diffusion spectaculaire des témoignages, notamment dans les pays anglo-saxons. Ce mouvement, plus tardif en France, émerge avec les publications du très médiatisé Josef Schovanec (philosophe, écrivain et militant pour la dignité des personnes avec autisme). Ces ouvrages représentent un matériau riche pour saisir les spécificités des troubles autistiques, confirmer des pistes de recherche et apporter un regard nouveau sur le phénomène. Les témoignages rédigés par des parents d'enfants avec autisme vont également se révéler être une précieuse source d'information. En complément des ouvrages spécialisés (médicaux, psychologiques, psychiatriques, etc.), ils fournissent une aide conséquente et éclairent effectivement le quotidien et la diversité des expériences familiales.

Dans le cadre d'une thèse de doctorat en sociologie, qui portait sur les socialisations alimentaires d'enfants présentant des troubles autistiques (Rochedy, 2017), nous avons procédé à l'analyse de biographies de parents d'enfants avec autisme. Ceci a complété des entretiens semi-directifs réalisés avec des mères et des pères. Le choix de cette méthodologie s'explique par plusieurs raisons. Tout d'abord, elle a

42. Traduction effectuée par nos soins. 
permis de répondre à une critique régulièrement formulée par les professionnels de l'autisme lors de restitutions des données de la phase qualitative. Selon eux, la grille d'entretien portant principalement sur les faits alimentaires, il était «normal» que les parents s'attardent et considèrent ces aspects ${ }^{43}$. Alors que, toujours selon eux, d'autres dimensions difficiles du quotidien devaient être privilégiées, notamment celles relatives aux critères d'identification de l'autisme ${ }^{44}$, l'analyse biographique a permis de recueillir des discours sans intervention du chercheur à propos des pratiques alimentaires de leur enfant et de l'incidence sur celles de leur famille. Ensuite, les témoignages de parents ont été privilégiés à d'autres sources, tels que les livres écrits par des personnes avec autisme ou les films et séries télévisées consacrés à cette cause, où c'est une image essentiellement réduite à l'autisme sans déficience intellectuelle qui est véhiculée : le fameux Asperger, un génie érudit «seulement» handicapé du point de vue de ses interactions sociales. L'objectif, au contraire, était de sonder la diversité des formes et des expériences de l'autisme. Enfin, les témoignages de parents s'étant multipliés depuis les années 2000, ils offraient une grille de lecture très diversifiée du vécu familial de l'autisme, un corpus conséquent et original donc $\mathrm{c}^{45}$.

Cette recherche documentaire a débuté dans les bases de données électroniques «classiques»: Francis, PubMed et Web of science. Les résultats n'ont toutefois pas été concluants du fait d'une littérature scientifique peu étoffée dans ce domaine. C'est plutôt la base de données du centre Ressources autisme MidiPyrénées qui nous a permis de repérer les ouvrages rédigés par des parents, ainsi que certains moteurs de recherche plus généralistes ${ }^{46}$. Notre recherche s'est focalisée sur les témoignages publiés en français par des parents entre 2008 et 2013. Ont été exclus de notre base de données les témoignages publiés par la fratrie des enfants avec autisme ou par les grands-parents, les récits à deux voix en dehors de ceux écrits par les seuls parents (par exemple, parent et enfant avec autisme ou parent et professionnel de santé), les guides de parents et les bandes dessinées ${ }^{47}$. L'objectif était de se concentrer uniquement sur les récits d'expériences vécues par l'entourage nourricier parental.

43. La problématique alimentaire est très souvent associée aux troubles autistiques. Néanmoins, selon les études, la fréquence des "problèmes alimentaires» est hétérogène du fait de l'absence de définition de cette notion et de la pluralité des disciplines qui s'intéressent à cette question (Rochedy, 2017). Il peut s'agir de la sélectivité alimentaire (consommation d'une petite variété d'aliments, choix répétitif, préférences marquées, refus d'aliments nouveaux, etc.), de sensibilité sensorielle, de problèmes de comportements sociaux à table ou encore de problèmes plus spécifiques (problèmes de mastication, mérycisme, dysphagie, etc.).

44. Il s'agit des critères d'évaluation de diagnostic qui composent la dyade autistique dans le DSM-5 : déficits de la communication et des interactions sociales, caractère restreint et répétitif des comportements, des intérêts.

45. Bien sûr, nous rappelons que nous avons conscience d'une limite de l'utilisation de ce type d'outil de seconde main. Lécriture d'un témoignage demande des capacités de rédaction, du temps et par conséquent ce corpus n'est pas représentatif de l'ensemble de la population des parents ayant un enfant présentant des troubles autistiques.

46. Les principaux mots-clefs étaient : «autisme», «troubles envahissants du développement», «troubles du spectre autistique», «témoignages», «livres», «parents».

47. Les guides et les bandes dessinés publiés par les parents ont été écartés car la nature des données fournies ne permettait pas «de comparer finement des itinéraires proches» (Peneff, 1990, p. 80) 
Au total, 29 ouvrages ont été retenus. Ils ont été publiés par des éditeurs tels qu'Albin Michel ou Robert Laffont, ce qui confirme la démocratisation du phénomène. Ces histoires se déroulent principalement en France, mais aussi dans d'autres pays et par conséquent d'autres cultures alimentaires sont présentes : Québec, États-Unis, Norvège, Italie, Grande-Bretagne et Pays-Bas. Les témoignages sont écrits en majorité par les mères, le reste étant pour moitié par les pères, et pour moitié par les deux parents. L'ensemble porte sur 28 familles $^{48}$ dont les situations familiales sont diversifiées et le nombre d'enfants oscille entre 1 et 3 . Au total, 33 enfants avec autisme sont présents dans ces témoignages; il s'agit majoritairement de garçons, répartition conforme aux données de prévalence. Les enfants présentent des formes très variées du spectre autistique, mais le diagnostic précis n'est pas toujours clairement énoncé. L'une des limites de l'utilisation de la biographie comme méthode d'enquête réside justement dans la difficulté, voire l'impossibilité de contacter les personnes pour recueillir des informations manquantes ou incomplètes.

Une fois identifiés, les 29 ouvrages ont été analysés avec une grille de lecture thématique diachronique et synchronique. Ces deux approches ont permis d'appréhender les faits alimentaires des enfants avec autisme dans leur complexité. En effet, il s'agissait de mieux décrire et comprendre la socialisation alimentaire de ces enfants, de la naissance à l'adolescence, en analysant simultanément la construction du répertoire alimentaire, les évolutions des apprentissages sociaux des manières de table et la gestion quotidienne de l'alimentation par l'entourage nourricier. La diversité des profils et des expériences vécues a permis d'éclairer certains aspects peu abordés dans la littérature scientifique et d'enrichir les thématiques abordées lors des entretiens semi-directifs. L'analyse thématique a révélé des temporalités hétérogènes, d'une part quant aux trajectoires alimentaires des enfants, d'autre part quant au travail parental effectué. En effet, certains ont construit leur récit entre «l'avant» et «l'après » diagnostic, quand d'autres relatent leurs expériences parentales sur une plus longue période, de la naissance de leur(s) enfant(s) jusqu'au moment de l'écriture de l'ouvrage. Par conséquent, plusieurs mouvements d'intensification et de transformation de la réflexivité alimentaire ont pu être identifiés. En reconstruisant leur propre histoire de vie, ainsi que celle de leur enfant avec autisme, les parents donnent sens aux difficultés rencontrées et transforment une expérience individuelle en expérience collective.

Cette méthode comporte toutefois des biais. D'une part, les récits parentaux sont parfois lacunaires : les caractéristiques sociodémographiques des enquêtés ne sont pas systématiquement renseignées et les contextes ne sont pas toujours précisément décrits. Par exemple, les parents ne mentionnent pas toujours l'âge des enfants quand ils racontent un événement, ils ne décrivent pas nécessairement la composition des repas ni la présence et le rôle des convives. D'autre part, les données permettant d'étudier la socialisation alimentaire des enfants avec autisme portaient essentiellement sur les temps alimentaires familiaux. Il aurait pourtant été utile de recueillir des éléments permettant de comprendre ce qu'il se passait dans d'autres univers de socialisation comme la crèche ou l'école. Il nous semble donc que cet outil, si pertinent soit-il à mobiliser, trouve son utilité et sa légitimité en complément d'autres méthodes d'enquête.

48. Une famille a publié deux livres : le premier (2008) a été publié en couple alors que le second (2012) l'a été seulement par le père. 


\section{Quel futur pour la méthode lauto-Jbiographique?}

Il nous semble utile, en guise de conclusion, d'envisager le futur de la méthode (auto-)biographique. De fait, de nombreuses innovations techniques voient le jour et pourraient en reconfigurer la forme et la pratique. Il s'agit par exemple de la reconnaissance vocale, qui permet de retranscrire aisément des récits oraux; du mode vidéo des smartphones, grâce auquel les individus se mettent en scène; ou encore de certaines applications mobiles pour établir des témoignages photographiés de tranches de vie. Ces diverses innovations remettent potentiellement en cause l'existence même de la méthode, incarnant le fait que l'entreprise (auto-) biographique opère de plus en plus quotidiennement par le biais de ces médias, au détriment de l'écriture. Même si l'on ne se raconte probablement pas de la même manière à l'oral qu'à l'écrit, il serait toutefois délétère, nous semble-t-il, de ne pas considérer les avantages que peuvent apporter ces outils. D'une part, ils pourraient contribuer à démocratiser quelque peu la méthode, notamment en la rendant plus accessible à des personnes issues de catégories sociales où l'écriture peut constituer une violence symbolique. D'autre part, ils pourraient aussi compléter la phase d'écriture, voire s'y substituer et ainsi constituer un support utile à la réalisation d'entretiens biographiques.

\section{W Références}

Bertaux D., 1997. Le récit de vie, Paris, Nathan, 127 p. (coll. 128).

Bossard C., Escalon H., Julia C., Poulain J.-P., Beck F., 2010. Mode de recueil et catégorisation des aliments : constances et évolutions méthodologiques, in Escalon H., Bossard C., Beck F. (éd.), Baromètre Santé Nutrition 2008, Bobigny, Inpes, 65-74.

Bourdieu P., 1986. Lillusion biographique. Actes de la recherche en sciences sociales, 62-63, 69-72. https://doi.org/10.3406/arss.1986.2317

Collectif B., 2020. Parler de soi. Méthodes biographiques en sciences sociales. Paris, Éditions de l'EHESS, 314 p. (coll. En temps \& lieux).

Heinich N., 2010. Pour en finir avec l'«illusion biographique». L'Homme, 3, 421-430.

https://doi.org/10.4000/lhomme.22560

Lamine C., 2008. Les intermittents du bio : pour une sociologie pragmatique des choix alimentaires émergents, Paris, Éditions de la Maison des Sciences de l'Homme/Quæ, 344 p. (coll. Natures sociales).

Parsons J. M., 2015. Gender, class and food. Families, bodies and health, Basingstoke, Palgrave Macmillan, 195 p. (coll. Family and Intimate Life).

Peneff J., 1990. La méthode biographique, Paris, Armand Colin, 144 p. (coll. U).

Rochedy A., 2017. Autismes et socialisations alimentaires. Particularités alimentaires des enfants avec un trouble du spectre de l'autisme et ajustements parentaux pour y faire face, Thèse de sociologie, Toulouse, Université de Toulouse Jean-Jaurès, 720 p.

Thomas W. I., Znaniecki F., 1998 [1918-1920]. Le paysan polonais en Europe et Amérique. Récit de vie d'un migrant, Paris, Nathan, 446 p. (coll. Essais \& Recherches). 



\title{
Chapitre 10 \\ La méthode follow-the-thing : pour une biographie sociale et spatiale des aliments
}

\author{
MICHAËL BRUCKERT ${ }^{a}$ \\ a CIRAD, UMR Innovation, Ha Noi, Viêt Nam ; Innovation, université de Montpellier, CIRAD, INRAE, \\ Institut Agro, Montpellier, France.
}

Ce chapitre expose une méthodologie d'investigation des faits alimentaires développée par la géographie humaine anglophone, l'approche dite follow-the-thing, qui vise à suivre les aliments dans l'espace afin d'en proposer des biographies sociales et spatiales. Cette approche rend visible les acteurs impliqués dans la circulation des aliments et caractérise leurs différents rôles. Elle permet aussi d'explorer les imaginaires géographiques des mangeurs, les représentations que ceux-ci ont de l'origine des aliments et l'impact de ces connaissances sur leur rapport à ces aliments.

Pendant longtemps, la géographie humaine s'est surtout intéressée à l'alimentation par le biais de l'étude de la production, des paysages agricoles et des terroirs. Ce n'est qu'à partir de la deuxième moitié $\mathrm{du} \mathrm{Xx}^{\mathrm{e}}$ siècle que la discipline se tourne davantage vers la question des pratiques alimentaires. En France, Max Sorre s’intéresse aux régimes alimentaires dans les années 1950. Aux États-Unis, Frederick Simoons travaille sur les proscriptions alimentaires dans les années 1960. Dans cette approche géoculturelle, les pratiques alimentaires sont cartographiées et des facteurs explicatifs de ces pratiques sont identifiés. Pour autant, les géographes ont tardé à proposer une méthodologie robuste pour l'études des comportements alimentaires. Si, en France, l'approche culturelle en géographie se tourne vers une étude de la gastronomie et des «cultures alimentaires » (Fumey, 2010) à partir des années 1990, c'est dans le monde de la géographie anglophone qu'à la même époque la consommation alimentaire quotidienne devient un sujet d'investigation, sous l'influence notamment d'un tournant culturel qui donne la part belle aux représentations et aux significations dans l'étude du champ social. Paradoxalement, cette approche dite culturelle, telle qu'elle se développe dans le monde académique anglophone, est beaucoup plus politique et sociale que son pendant français, à résonance plus patrimoniale.

Dans les années 1980, sous l'influence de ce tournant culturel, se développe dans le monde anglophone (et principalement en Grande-Bretagne) une géographie de la consommation cherchant à élargir le champ d'investigation de la géographie 
économique au-delà de la simple sphère de production. C'est dans ce contexte que des géographes vont proposer des modalités d'étude du caractère spatial de la consommation alimentaire. L'espace n'est alors plus uniquement considéré comme un support physique neutre sur lequel se déploieraient des filières d'approvisionnement régies par des contraintes politiques et économiques, mais aussi comme une ressource matérielle et idéelle pour les mangeurs et les autres acteurs du système alimentaire.

Une première approche développée par David Bell et Gill Valentine s’intéresse plus spécifiquement au cadre géographique de la consommation alimentaire. Dans leur ouvrage Consuming geographies (1997), les deux géographes britanniques proposent une lecture multi-scalaire de la consommation. En prenant en compte les échelles du corps, du foyer, de la communauté, de la ville, de la nation et du monde, ainsi que les multiples articulations entre ces échelles, ils se concentrent sur les configurations spatiales et les itinéraires sociaux qui sont établis à travers les usages des aliments.

\section{W Lapproche follow-the-thing}

Une deuxième approche proposée aux débuts des années 2000 s'intéresse plus aux aliments qu'aux mangeurs. Cette méthodologie suggère de suivre les aliments, de les accompagner dans leur cheminement à travers l'espace et d'en proposer une sorte de géobiographie. Elle est théorisée sous l'expression follow-the-thing et s'applique par la suite à l'étude des objets non alimentaires. Un ouvrage fondateur a fortement inspiré les concepteurs de cette approche : The social life of things, dirigé par Arjun Appadurai en 1986. Dans son chapitre introductif (Appadurai, 1986), l'anthropologue s'appuie sur les travaux de Karl Marx (notamment la théorie du fétichisme de la marchandise), de Georg Simmel (théorie de la valeur et de l'argent), de Jean Baudrillard (sur les objets et la consommation comme une manipulation de signes) ou de Mary Douglas et Baron Isherwood (sur les marchandises et la consommation) pour proposer une théorie sur la circulation des marchandises dans la société. Pour Appadurai, les marchandises n'ont pas de signification en soi : ce sont les humains qui leur attribuent des significations particulières par leurs transactions. Dans la mesure où les «choses-en-mouvement» (things in motion) peuvent «éclairer leur contexte humain et social» (Appadurai, 1986, p. 5), il propose comme méthodologie d'enquête de «suivre les choses elles-mêmes, car leurs significations sont inscrites dans leurs formes, dans leurs usages, dans leurs trajectoires» (ibid.).

Dans les années 1990, Ian Cook, un géographe britannique, cherche à concilier l'approche d'Appadurai à une approche plus classique d'étude de filières (commodity chains) telle qu'elle est couramment pratiquée en géographie. Avec son collègue Phil Crang, ils proposent de s'intéresser à la «vie sociospatiale» des aliments ou, plus spécifiquement, de comprendre comment les flux et les réseaux globaux d'aliments, de personnes et de savoirs culinaires s'articulent dans des lieux et des situations particulières (Cook et Crang, 1996). Cook et ses collègues proposent ensuite d'acquérir une «compréhension biographique et géographique des aliments» (Cook et al., 1998, p. 162). Documenter les vies des aliments permet de mettre en lumière les relations dialectiques qui unissent l'alimentation et l'espace : nos choix alimentaires sont affectés par des pratiques situées à distance de notre expérience directe et, en 
retour, ces choix ont des effets sur des lieux situés au-delà de la sphère domestique de consommation. Dans une référence explicite à Appadurai, Peter Jackson propose quant à lui de «tracer une géographie sociale des choses» (Jackson, 1999, p. 104), et notamment des aliments. Paul Robbins fait de même, affirmant que «des processus sociaux, politiques et culturels investissent les objets de significations [...] à mesure que l'échange les déplace dans et hors de situations socialement et politiquement définies » (Robbins, 1999, p. 401).

On notera que cette approche se retrouve déjà sous une certaine forme dans l'ouvrage majeur de Sydney Mintz, Sweetness and Power (Mintz, 1985; traduction française: La douceur et le pouvoir). Dans son étude de l'histoire du sucre, l'anthropologue considère que production et consommation sont étroitement liées, et que les transformations dans l'usage de cet aliment ont entraîné des changements dans les significations qui lui sont attribuées. Néanmoins, dans son ouvrage, Mintz fait œuvre d'historien et «suit» le sucre de façon virtuelle, à partir d'archives et de sources secondaires.

À la fin des années 1990, la méthodologie de «suivre les choses» n'est pas encore théorisée. Si certains géographes l'emploient, ce n'est que dans les années 2000 que Cook publie des articles consacrés à cette méthodologie à proprement parler : d'abord à son application (Cook, 2004), puis à sa théorisation (Cook et al. $\left.{ }^{49}, 2006\right)$.

\section{Premières esquisses et mise en application}

Dans son article de 2004, intitulé «Follow the Thing: Papaya», Cook s'appuie ouvertement sur l'approche marxiste en géographie (développée notamment par David Harvey), laquelle suggère de «dé-fétichiser les marchandises » (Cook, 2004, p. 642) : quand il arrive dans l'assiette du consommateur, le produit est accompagné de significations qui masquent les véritables conditions socio-économiques qui ont présidé à sa production. Les valeurs conférées aux aliments sont posées comme naturelles par les mangeurs, alors que ce sont les humains qui leur donnent ces valeurs par leur travail : les rapports sociaux de production sont occultés dans la relation des mangeurs à leurs aliments. Pour lever le voile sur ce fétiche, Cook suit l'itinéraire qu'empruntent des papayes produites en Jamaïque et expédiées dans les supermarchés britanniques. Il interroge des cultivateurs, des contremaîtres, des acheteurs, des empaqueteurs, des importateurs et des mangeurs pour documenter une biographie sociale et spatiale des papayes. Il croise cette biographie à des considérations sur l'économie politique de la papaye, son histoire, ses trajectoires (routes) mondiales, sa consommation et sa fétichisation par la publicité.

Son article se veut ouvertement novateur : le ton est non académique et le récit brise la linéarité de la chaîne d'approvisionnement, proposant une collection de vignettes plus qu'une argumentation méthodique. En exposant la façon dont le commerce mondial de la papaye est «enchevêtré dans des processus économiques, politiques, sociaux, culturels et agricoles» (Cook, 2004, p. 642), l'article vise également à provoquer des questionnements moraux et éthiques sur la connexion entre producteurs et consommateurs.

49. Ian Cook signe tous ses articles «Cook et al.» : c'est une façon pour lui de dire qu'il n'est jamais seul dans l'écriture d'un article. 


\section{Une théorisation plus systématique}

En 2006, dans un article intitulé «Geographies of Food: Following», Cook expose plus longuement l'ancrage théorique de cette approche. D'après lui, en «suivant les aliments» et en «racontant des histoires avec eux» (Cook et al., 2006, p. 657), les chercheurs peuvent mettre en lumière à la fois les pratiques des acteurs du système alimentaire, la façon dont différentes significations sont conférées à un aliment par différents acteurs et en différents lieux au sein de ce système, ainsi que les relations entre ces pratiques et ces significations. Les valeurs attribuées aux aliments revêtent ainsi une forme de «dynamique spatiale» (Coles, 2013, p. 259).

Cette méthodologie répond à un questionnement très présent dans la géographie humaine anglophone à cette époque : comment associer dans l'analyse les aspects économiques et les aspects culturels des marchandises? Le postulat de départ est que l'économique et le culturel sont mutuellement constitués : les pratiques, catégories et valeurs économiques sont informées par des représentations et des significations culturelles particulières et, inversement, les représentations culturelles sont affectées par les pratiques et valeurs économiques (Freidberg, 2004, p. 9).

D’une façon générale, la démarche follow-the-thing combine une approche d'économie politique, qui décrit les relations sociales de production et l'extraction de la plus-value tout au long de la chaîne d'approvisionnement, et une approche culturelle qui se veut poststructuraliste. Selon cette dernière approche, les pratiques et les énoncés des acteurs sociaux (et plus spécifiquement des consommateurs) ne sont ni préétablis par des structures socialement constituées, ni directement déterminés par l'organisation de la production : ils sont toujours soumis à l'interprétation, à la négociation et à l'expérience des acteurs individuels. Plus spécifiquement, il s'agit pour Cook de concilier deux approches : une démarche marxiste et la théorie de l'acteur-réseau (actor-network theory). La première considère classiquement que la production produit «l'objet de la consommation, mais aussi le mode de consommation» (Marx 1993, p. 44). La seconde, alors très en vogue dans la géographie anglophone, rejette le dualisme entre «nature» et «société» pour proposer d'étudier la façon dont des entités humaines et non humaines s'agencent en réseaux (Latour, 1996). Ce faisant, elle vise à conférer une certaine forme de capacité d'action (agency) aux objets étudiés - par exemple, pour la papaye et le papayer, nous dit Cook (Cook et al., 2006, p. 650), le fait de changer le sexe de sa fleur, de sécréter des enzymes, de s'abîmer, etc. Par ailleurs, la théorie de l'acteur-réseau s'intéresse aux intermédiaires non humains (contrats, régulations, conventions, etc.) qui relient les acteurs et permettent à un acteur d'agir à distance sur un autre acteur.

Cook rappelle que l'enjeu de cette approche est également d'ordre éthique et politique : «suivre les choses» doit permettre de mettre en lumière les différentes formes d'exploitation économique dans les systèmes alimentaires et de penser aux conditions d'émergence de réseaux d'approvisionnement alternatifs qui reconnecteraient les différents acteurs et remédieraient à la fragmentation des savoirs géographiques. Dans cette reformulation méthodologique, et pour répondre à certaines critiques, Cook précise que le fétiche qui enveloppe la marchandise et masque les relations de production ne doit pas tant être levé dans une opération 
quasiment épiphanique de dévoilement et d'accès à la chose même, mais qu'il doit plutôt être saisi, affronté, explicité. L'enjeu est alors d'opposer des récits alternatifs aux récits officiels, lesquels mettent en lumière certains lieux et certaines pratiques (par exemple le vert pâturage où paissent les bovins), tout en en masquant volontairement d'autres (par exemple l'abattoir).

Par ailleurs, «suivre les choses» doit non seulement permettre de gagner en connaissance, mais également en empathie et en compréhension pour celles et ceux dont la vie est liée à la nourriture que nous achetons et mangeons, nous dit Cook (Cook et al., 2006, p. 660). Il conclut son article en appelant de ses vœux l'émergence d'études de l'alimentation (food studies) qui soient radicales et post-disciplinaires.

Cette méthodologie a donné lieu à un site Internet ${ }^{50}$, animé par Ian Cook et ses étudiants. L'objectif de ce site, qui recense des travaux (mémoire d'étudiants, documentaires, articles de recherche, livres, etc.) mettant en œuvre cette approche d'une façon plus ou moins revendiquée, est clairement à visée éthique et pédagogique : il s'agit de montrer les ingrédients cachés des produits de consommation courante et d'exposer leurs conditions de production.

\section{$\leadsto$ À la croisée des aliments, des lieux et des mangeurs}

L'aliment est central dans cette approche. Néanmoins, le mangeur n'est pas négligé : l'acte de consommation est envisagé du point de vue d'un produit, dont les significations sont construites et négociées tout au long du système alimentaire. Ces significations ne sont ni des attributs naturels ou intrinsèques qui y seraient matériellement fixés par le processus de production, ni le produit d'une culture englobante qui viendrait de l'extérieur se plaquer sur des objets. Au contraire, elles sont produites dans différents lieux en relation, et elles circulent grâce à plusieurs processus et à diverses pratiques. À mi-chemin entre matérialisme ${ }^{51}$ et culturalisme $^{52}$, cette approche permet d'explorer les imaginaires géographiques des mangeurs, les représentations que ceux-ci ont de l'origine des aliments, et l'impact de ces connaissances sur leur rapport à ces aliments. Cette méthodologie permet donc une approche dialectique des rapports et des transactions matériels et sémiotiques qui relient les différentes composantes des systèmes alimentaires (production, transformation, distribution, consommation, régulation, etc.).

Cette méthodologie met aussi en lumière les rapports de pouvoir et de domination qui président à l'attribution de significations. Plus les systèmes d'approvisionnement sont étendus, plus nombreuses sont les opportunités de négociation et de confrontation au sujet de ces significations. Cette approche peut notamment éclairer les changements brutaux de significations qui peuvent survenir quand un aliment passe d'une main à une autre, ou plus généralement les différentes significations que producteurs, transformateurs, distributeurs et mangeurs attribuent au même produit.

50. http://www.followthethings.com/ (dernière consultation le 28/08/2021).

51. Approche épistémologique qui s'intéresse avant tout à l'aspect matériel des objets étudiés.

52. Approche épistémologique qui s'intéresse aux objets étudiés avant tout à travers les symboles et les discours qui y sont associés. 
Méthode lancée par des géographes, l'approche follow-the-thing interroge également les liens entre les lieux, les corps des mangeurs et les aliments. Par l'alimentation, les lieux ne sont pas uniquement connectés de façon linéaire. Pour le géographe Benjamin Coles, un aliment contient toujours une part du lieu dans lequel il a été produit et dans lequel il est consommé : les lieux sont «encastrés» (embedded) et «incarnés» (embodied) dans la substance même des aliments (Coles, 2013, p. 256). Ces lieux sont définis et produits par une interaction entre des caractéristiques matérielles, sociales et discursives, mais également par des interconnexions entre différents lieux. Ingérer un aliment, c'est ingérer une part de ces lieux, au sens matériel, mais également au sens discursif. En d'autres termes, manger un produit, c'est manger sa géographie - définie comme un «agencement de lieux» (Coles, 2013, p. 257). L'approche followthe-thing permet donc d'examiner la façon dont différents lieux s'incarnent dans les aliments et dont ils sont ensuite matériellement et symboliquement ingérés par les mangeurs. Les «géographies incarnées du café» qu'expose Coles, en s'appuyant ouvertement sur la méthode follow-the-thing, sont éclairantes à cet égard.

Cette méthodologie comporte donc deux dimensions : une dimension ontologique - en affirmant que les aliments sont attachés à de multiples lieux eux-mêmes en relation, et qu'ils prennent leur sens en lien avec ces lieux - et une dimension épistémologique - en considérant que c'est en suivant les aliments dans l'espace et en enquêtant dans ces multiples lieux que les rapports des mangeurs à ces aliments peuvent être éclairés.

\section{" Une ethnographie multi-site}

La mise en œuvre de la méthodologie n'est pas documentée de façon très détaillée dans la littérature. Principalement, le protocole d'enquête consiste en une ethnographie multi-site - donc par une approche résolument qualitative. Cook considère que le caractère multi-site est approprié pour déchiffrer la mondialisation des systèmes alimentaires, mais également que seule l'observation participante permet de comprendre les vies des producteurs, des transformateurs, des intermédiaires et des mangeurs. Il va cependant plus loin, appelant les investigateurs à une forte réflexivité dans leurs pratiques : dans une forme de narration auto-ethnographique (Cook et al., 2006, p. 660), les émotions suscitées par les découvertes faites, ainsi que la façon dont ces découvertes altèrent les propres connaissances et convictions de l'enquêteur, doivent être exposées. Cook donne peu de détails au sujet de la méthodologie d'enquête à proprement parler. Les différents auteurs qui ont mis en œuvre cette méthode favorisent les approches qualitatives et participatives, donnant la part belle aux histoires de vie des personnes interrogées. À l'échelle des mangeurs, les principales questions portent sur leurs imaginaires géographiques, sur leurs connaissances quant aux origines et modes de production des aliments, ou sur l'influence de ces connaissances sur leurs modes de consommation. À l'évidence, l'ethnographie de terrain doit être complétée par une recherche documentaire concernant les caractéristiques matérielles des aliments étudiés, la structuration des réseaux d'approvisionnement et leur évolution historique, les textes législatifs et réglementaires régissant leurs échanges et usages, les discours médiatiques ou publicitaires les évoquant, les éventuels débats de société à leur sujet, etc. Les sources de première main et de seconde main se complètent. 


\section{- Limites et points de vigilance}

Ciblée sur un aliment particulier et sur sa trajectoire sociospatiale, cette méthodologie ne permet pas de rendre compte de l'intégralité des pratiques des mangeurs : elle éclaire le rapport à un aliment spécifique qui a été suivi dans l'espace, mais pas le régime alimentaire d'un mangeur au sens large et le sens qu'il donne à son alimentation.

Par ailleurs, l'emploi de la notion marxiste de «fétichisme de la marchandise» a pu être considéré comme trop simpliste par certains auteurs. Premièrement, le fétiche attaché à une marchandise n'est pas le produit d'une intention de masquer sa réalité, mais plutôt l'effet inévitable du système d'échange marchand (Goss, 2004). Pour les marxistes, il serait bien illusoire de penser pouvoir s'en débarrasser en le mettant en lumière : seule l'abolition du mode de production capitaliste pourrait venir à bout de ce fétichisme. Deuxièmement, l'idée qu'il suffirait de dévoiler les conditions de production d'un aliment pour changer le comportement d'achat peut être considérée comme une approche moralisatrice, élitiste, voire comme une légitimation d'une économie néolibérale de marché dans laquelle le consommateur serait un acteur qui agirait de façon rationnelle s'il était correctement informé.

L'ethnographie multi-site, sur laquelle s'appuie l'approche follow-the-thing, est en elle-même une méthodologie qu'il faudrait exposer en détail. Principalement, les auteurs qui emploient l'approche follow-the-thing font référence à l'article fondateur de Marcus (1995), lequel considérait dans les années 1990 l'ethnographie multi-site comme une méthode interdisciplinaire appropriée pour prendre en compte l'articulation du local et du global dans les faits sociaux. Plus récemment, le sociologue Jean-Pierre Hassoun a proposé l'expression d' «observation circulante ( Hassoun, 2020, p. 130) afin de mieux prendre en compte la circulation des objets, des personnes et des représentations entre les différents lieux étudiés.

Finalement, l'approche follow-the-thing peut apparaître un peu dépassée dans les années 2020. Née à une époque où les systèmes alimentaires étaient encore en voie d'être mondialisés, elle n'est plus forcément appropriée à l'extrême diversité des réseaux d'approvisionnement actuels : elle est difficile à adapter à des aliments composés et ultra-transformés, ou dont les trajectoires spatiales sont trop complexes, fragmentées, ramifiées, changeantes, ou ponctuées par des ruptures (Hulme, 2017). Par ailleurs, elle ne paraît plus novatrice, tant se sont développés depuis lors les documentaires télévisés et articles de presse rendant visibles les coulisses de la mondialisation, les applications informatiques permettant d'obtenir des informations sur les aliments achetés ou les emballages de produits équitables arborant des visages de producteurs supposément émancipés et heureux. Néanmoins, cette inflation dans les informations communiquées par les entreprises agroalimentaires peut être considérée comme un nouveau régime de fétichisation des produits : l'approche follow-the-thing retrouverait alors tout son intérêt pour dévoiler les zones d'ombre de cette prétendue mise en lumière opérée par la communication.

\section{W Aspects éthiques et déontologiques}

Cette approche ne pose pas de question déontologique particulière, hormis celles habituellement posées par l'enquête ethnographique. Une vigilance particulière doit être observée à l'égard des informations personnelles recueillies lors des entretiens 
avec les différents acteurs (producteurs, consommateurs, etc.), ainsi que des informations confidentielles (secret industriel, etc.) qui pourraient être transmises, comme cela est parfois le cas dans l'étude des systèmes alimentaires. Comme toute démarche de recherche en sciences humaines, elle est soumise aux législations en vigueur dans l'espace où les enquêtes sont conduites et les données traitées (le règlement général sur la protection des données, ou RGPD, dans l'Union européenne par exemple).

\section{" Un positionnement multidisciplinaire}

En développant l'approche follow-the-thing pour l'étude de l'alimentation, la géographie approfondit la vocation systémique et le positionnement multidisciplinaire qu'elle a souvent revendiqués. Cette approche permet d'aller à l'encontre d'une conception linéaire d'une chaîne ou d'une filière d'approvisionnement dont la consommation ne serait que le point final. Elle ne détache pas les mangeurs du système qui permet de produire, transformer et acheminer des aliments, mais qui permet également, sur un mode plus discursif, de faire circuler les informations, les énoncés et les significations relatifs à ces aliments.

L'approche permet donc de dépasser à la fois l'opposition entre production et consommation dans l'étude de l'alimentation, mais également la dichotomie entre le matériel et le discursif, entre les pratiques et les significations. C'est donc une méthodologie holistique qui s'appuie sur une ontologie fondamentalement relationnelle : les différentes composantes des systèmes alimentaires doivent être étudiées ensemble, et les aspects matériels ne peuvent être disjoints des aspects sémiotiques. Plus largement, cette démarche semble appropriée pour appréhender les changements ayant cours dans les systèmes alimentaires. Elle permet d'intégrer dans l'analyse la prise en compte des processus écologiques, des infrastructures matérielles, des réseaux économiques, des acteurs politiques et des dispositifs symboliques qui, tous ensemble, contribuent à définir et à réguler la présence, la circulation, la visibilité, les statuts et les multiples propriétés prêtées aux différents aliments.

À l'évidence, cette démarche n'est pas purement géographique. Réduire le fait alimentaire à une simple question de localisation des aliments reviendrait à une forme de réductionnisme spatial qui nierait la substance des faits sociaux. La méthode suppose donc de mobiliser parallèlement d'autres outils des sciences sociales, et notamment la démarche socio-ethnologique : elle seule permet de rendre compte des pratiques observées, des discours entendus, et ainsi d'éclairer les pratiques alimentaires et de les interpréter comme de véritables faits sociaux sous-tendus par des logiques qui leur sont propres. Ainsi, cette méthode est assez peu technique et reste large dans sa définition : elle peut être mobilisée par toute personne ayant des connaissances générales en sciences sociales. En soi, elle est fondamentalement multidisciplinaire.

\section{" La viande en Inde : un exemple de biographie spatiale d'un aliment}

La mise en œuvre de la méthodologie consiste principalement à documenter les pratiques et les représentations des acteurs sur différents lieux du système alimentaire, en s'intéressant notamment aux changements qui affectent les significations attribuées 
à l'aliment étudié quand celui-ci est échangé et circule à travers l'espace. C'est cette méthode que j'ai appliquée lors d'une recherche doctorale qui portait sur les pratiques et les significations relatives à la viande dans l'État du Tamil Nadu (Inde du Sud), et dont les résultats ont ensuite été publiés dans un ouvrage (Bruckert, 2018).

L'objet de cette recherche était d'éclairer les reconfigurations des statuts symboliques et juridiques des différentes viandes (poulet, bœuf, viande caprine et viande ovine) dans un contexte de mondialisation, d'urbanisation, de montée en puissance du nationalisme hindou et d'émergence de questions écologiques et sanitaires. Principalement, j'ai cherché à mettre en lumière des circuits spécifiques de la viande, avec leurs modalités sociotechniques propres, et à interroger la façon dont les significations attribuées à la viande sont façonnées, contestées et reconfigurées tout au long de ces circuits. L'hypothèse de départ était que les significations attribuées par les mangeurs aux différentes viandes ne sont pas uniquement façonnées dans l'espace de consommation, mais également tout au long des circuits d'approvisionnement : les acteurs qui élèvent les bêtes, les échangent, les abattent, découpent les carcasses, vendent la viande, la servent au restaurant, mais aussi ceux qui rédigent les réglementations, produisent des expertises, font circuler des informations ou militent dans des groupes religieux, environnementaux et de protection animale - tous ces acteurs façonnent les significations de la viande, en lien ou non avec les attentes et les imaginaires des mangeurs. Tout au long des circuits, les demandes des mangeurs induisent des façons de travailler particulières et, en retour, les contraintes et les choix des acteurs des filières façonnent des pratiques de consommation spécifiques.

Dans cette recherche, la méthode employée était directement inspirée de l'approche follow-the-thing. Il s'agissait dans un premier temps d'interroger les différentes trajectoires de la viande dans l'espace social : où la viande est-elle située ? Où va-t-elle ? Où ne va-t-elle pas? Quelles sont les pratiques et les significations relatives à la viande en chacun des lieux de ces circuits et comment varient-elles? Une ethnographie multi-site a permis une étude des pratiques et des discours tout au long des circuits. Les lieux d'étude identifiés mettaient en jeu un gradient d'urbanité afin d'étudier les différentes étapes des circuits dans des contextes plus ou moins urbanisés. L'étude de terrain a duré environ un an, divisée en séjours de plusieurs mois. Mon point de départ était l'étude des boucheries urbaines, à la fois parce que leur accès était relativement aisé, mais aussi parce que le boucher est un médiateur : les boucheries sont le lieu crucial où se rencontrent la production et la consommation, où les mangeurs, les revendeurs et les législateurs négocient les significations attribuées à la viande. Des boucheries, mon chemin m'a conduit en amont de la chaîne aux abattoirs périurbains, et en aval aux restaurateurs et aux mangeurs. J'ai réalisé des entretiens semi-directifs avec plus de quatre-vingts mangeurs en tâchant de faire varier six critères principaux : le sexe, l'âge, la religion, la caste, la classe sociale et le lieu d'habitation. Les mangeurs ont été interrogés sur leur régime alimentaire, leur consommation de viande, leurs pratiques d'approvisionnement, l'influence du lieu (à la maison ou à l'extérieur) sur la consommation carnée, leurs représentations de la viande et leurs connaissances des circuits d'approvisionnement. Puis je suis parti au point de départ de la chaîne, en étudiant le travail des éleveurs à la lumière du produit final de cet élevage, à savoir la viande. De là, j'ai accompagné les animaux dans les marchés aux bestiaux et sur les routes les menant aux abattoirs et boucheries. Des experts, chercheurs, vétérinaires, responsables politiques, représentants 
professionnels et autres militants ont également été interrogés. Constamment, des allers-retours ont été effectués entre les différents lieux, notamment pour mieux saisir les connexions et les circulations entre ces différents espaces. La principale limite de cette approche était qu'elle risquait d'isoler la viande des autres produits alimentaires et de négliger le fait que les circuits d'approvisionnement et les comportements alimentaires sont un ensemble qui présente une certaine cohérence. Il a donc fallu questionner les mangeurs rencontrés sur leur alimentation de façon plus large, par-delà la simple question de la viande.

Les observations et les entretiens effectués en suivant les viandes ont mis en lumière les pratiques des acteurs et les logiques qui sous-tendent ces pratiques. Éleveurs, bouchers, abatteurs, marchands et restaurateurs ont été interrogés sur leurs pratiques et sur celles des mangeurs. Cette méthode a notamment permis d'identifier ce qui, dans les pratiques professionnelles, relève de contraintes techniques ou économiques et ce qui relève de pratiques culturelles ou éthiques. Par exemple, dans une optique d'anthropologie des techniques, l'étude des modalités de découpe de la viande dans les boucheries (conservation ou non du gras et de la peau, bris des os, désarticulation de la carcasse, etc.) a permis d'éclairer certaines pratiques et représentations des consommateurs et réciproquement. Ainsi, le drainage du sang, observé lors de l'abattage, a pu être considéré, en fonction des acteurs, comme une nécessaire conformité à une prescription religieuse, comme une garantie de sécurité sanitaire ou encore comme un gage de palatabilité. De même, la préférence exprimée par certains mangeurs pour la viande bovine ou bubaline a été questionnée au regard des contraintes économiques, agronomiques, ou juridiques qui pèsent sur l'élevage et l'abattage des bovins et des bubalins. Dans ce cas-là, il apparaissait que ce sont des conceptions d'ordre religieux plus que matériel ou économique qui déterminent les statuts symboliques et juridiques des viandes et des animaux. De façon peut-être plus éloquente, la distinction spatiale constatée dans les abattoirs entre une zone dédiée à l'abattage des bovinés et une zone dédiée à l'abattage des ovins et caprinés pouvait être associée à une nécessité de spécialisation technique et économique des activités, mais elle apparaissait principalement comme la traduction matérielle et spatiale d'un cloisonnement et d'une hiérarchisation entre deux types de viandes dont les statuts sont majoritairement jugés comme inégaux.

Le cas du poulet a été étudié en détail. Les mangeurs ont été questionnés sur leur perception de la chair de poulet et des risques liés à sa consommation, sur les processus d'activation de la confiance (visibilité des activités d'élevage et d'abattage, certifications, normes industrielles, etc.), sur leur connaissance des conditions de production, etc. J'ai suivi les poulets depuis les hangars d'élevage jusqu'aux réfrigérateurs des supermarchés en passant par les marchés de gros. Des entretiens ont été réalisés avec le patron d'une entreprise d'élevage avicole intégrée, des ouvriers saisonniers travaillant dans les exploitations, des bouchers artisanaux, des grossistes, des responsables de supermarchés et de magasins haut de gamme. Les supports de communication de l'industrie avicole et les articles de presse relatifs au poulet ont été étudiés. Cette méthodologie a permis de dessiner une biographie sociale et spatiale du poulet au Tamil Nadu et de documenter la façon dont la modification des conditions d'élevage et de transformation des viandes affectait ou non les représentations et les pratiques des mangeurs. Elle a mis en lumière le processus de désanimalisation qui affecte l'ensemble du circuit d'approvisionnement en poulet de chair : 
le caractère vivant des poulets est nié par l'intensification des pratiques d'élevage, tandis que l'abattage industriel et la vente de chairs découpées et congelées tendent à masquer l'origine animale de cette viande. Cette désanimalisation rend la chair de poulet plus acceptable pour de nombreux hindous; elle concourt à l'augmentation de sa consommation, mais elle génère également chez les mangeurs un sentiment de perte, une forme de nostalgie pour la chair ferme, goûteuse et considérée comme plus fortifiante des poulets de race locale élevés en plein air (Bruckert, 2021).

Par-delà la distinction entre différents types de viandes, la mise en œuvre de l'approche follow-the-thing m'a permis d'établir une typologie entre trois types de circuits de la viande qui répondent à des logiques spécifiques : un circuit vernaculaire, un circuit artisanal et un circuit de masse. Pour chacun de ces circuits, des caractéristiques ont été identifiées et mises en relation : l'extension spatiale, la division des tâches, les modalités d'élevage, la légitimation de la mise à mort, les logiques de partage de la carcasse, les usages culinaires et modalités de consommation et de classification des viandes, mais également des aspects plus généraux comme le rapport au monde animal que ces circuits reflètent et perpétuent, les connaissances que les mangeurs ont de ces circuits, et leur politisation par les partisans du nationalisme hindou ou leur régime de visibilité (tableau 10.1).

Tableau 10.1. Configurations sociospatiales des circuits de la viande en Inde (repris de Bruckert, 2018, p. 331-333).

\begin{tabular}{|c|c|c|c|c|}
\hline & & Circuit vernaculaire & Circuit artisanal & Circuit de masse \\
\hline \multirow{7}{*}{ 苋 } & $\begin{array}{c}\text { Animaux } \\
\text { emblématiques }\end{array}$ & Bouc, coq & Petit ruminant, poulet & Poulet de chair \\
\hline & $\begin{array}{c}\text { Rapport } \\
\text { au monde animal }\end{array}$ & $\begin{array}{l}\text { Continuité, proximité, } \\
\text { singularisation }\end{array}$ & $\begin{array}{l}\text { Discontinuité, } \\
\text { marchandisation }\end{array}$ & $\begin{array}{l}\text { Discontinuité, } \\
\text { chosification, } \\
\text { mythification? }\end{array}$ \\
\hline & Maîtrise du circuit & Groupe social/familial & Marchands, grossistes & Industriels \\
\hline & $\begin{array}{l}\text { Connaissances } \\
\text { du circuit par } \\
\text { les mangeurs }\end{array}$ & Très bonnes & Bonnes à moyennes & Faibles \\
\hline & $\begin{array}{c}\text { Origines } \\
\text { de l'imaginaire } \\
\text { lié au circuit }\end{array}$ & $\begin{array}{l}\text { Expérience, } \\
\text { connaissance directe }\end{array}$ & $\begin{array}{l}\text { Récits, souvenirs, } \\
\text { circulations }\end{array}$ & $\begin{array}{l}\text { Système industriel, } \\
\text { médias, champ politique }\end{array}$ \\
\hline & Politisation & Faible & Forte & Forte \\
\hline & $\begin{array}{l}\text { Localisation } \\
\text { et visibilité }\end{array}$ & Visibilité locale & Éloignement & $\begin{array}{l}\text { Invisibilisation et } \\
\text { banalisation }\end{array}$ \\
\hline \multirow{3}{*}{ 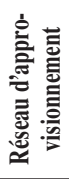 } & Extension spatiale & Faible & Moyenne à longue & Moyenne à très longue \\
\hline & $\begin{array}{l}\text { Modèle } \\
\text { technique }\end{array}$ & $\begin{array}{l}\text { Domestique } \\
\text { et extensif }\end{array}$ & Extensif et intensif & Intensif et industrialisé \\
\hline & Division des tâches & Faible & Moyenne & Importante \\
\hline \multirow{3}{*}{ 总 } & $\begin{array}{l}\text { Propriétaires } \\
\text { des animaux }\end{array}$ & Particuliers & Éleveurs & Entreprises privées \\
\hline & $\begin{array}{l}\text { Modalités } \\
\text { d'élevage }\end{array}$ & Diversifié, extensif & $\begin{array}{l}\text { Spécialisé, semi- } \\
\text { intensif, intensif }\end{array}$ & Professionnel, intensif \\
\hline & Relations de travail & Parenté & Caste, lignée & Salariat \\
\hline
\end{tabular}


Tableau 10.1. (suite)

\begin{tabular}{|c|c|c|c|c|}
\hline & & Circuit vernaculaire & Circuit artisanal & Circuit de masse \\
\hline \multirow{4}{*}{ 怤 } & $\begin{array}{l}\text { Légitimation } \\
\text { de l'abattage }\end{array}$ & Sacrifice, ritualisation & Ritualisation (halal) & Hygiène, halal? \\
\hline & Usage du sang & Offrande aux divinités & Évacuation & $\begin{array}{l}\text { Valorisation comme } \\
\text { sous-produit }\end{array}$ \\
\hline & Mise à mort & Exposée & Éloignée & Masquée \\
\hline & Viande de bœuf & Animaux «tombés» & Animaux de réforme & Bufflons engraissés? \\
\hline \multirow{2}{*}{ 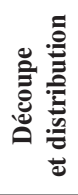 } & Logique de partage & Sociale, oblatoire & Économique & Économique \\
\hline & $\begin{array}{c}\text { Découpe de la } \\
\text { carcasse et } \\
\text { propriétés physiques } \\
\text { de la viande }\end{array}$ & $\begin{array}{l}\text { Carcasse fendue, bris } \\
\text { des os, viande fraîche }\end{array}$ & $\begin{array}{l}\text { Carcasse fendue, } \\
\text { morceaux, viande } \\
\text { fraîche }\end{array}$ & $\begin{array}{l}\text { Morceaux désossés, } \\
\text { prêts à cuire, prêts à } \\
\text { consommer, congelés, } \\
\text { réfrigérés }\end{array}$ \\
\hline \multirow{9}{*}{ 氠 } & $\begin{array}{c}\text { Modalité } \\
\text { de consommation }\end{array}$ & $\begin{array}{l}\text { Sacrificielle, } \\
\text { cérémonielle }\end{array}$ & $\begin{array}{l}\text { Cérémonielle, } \\
\text { banalisée }\end{array}$ & Banalisée \\
\hline & Usages culinaires & Ragoût & Ragoût, friture, pilaf & $\begin{array}{l}\text { Multiplicité des recettes, } \\
\text { répertoires extérieurs }\end{array}$ \\
\hline & $\begin{array}{c}\text { Lieux } \\
\text { de consommation }\end{array}$ & $\begin{array}{l}\text { Domicile, espace rural } \\
\text { extérieur }\end{array}$ & Domicile, restaurant & Domicile, restaurant \\
\hline & $\begin{array}{c}\text { Fréquence } \\
\text { de consommation }\end{array}$ & Mensuelle & Hebdomadaire & Quotidienne? \\
\hline & $\begin{array}{l}\text { Logiques présidant } \\
\text { à la consommation }\end{array}$ & $\begin{array}{l}\text { Sacrée, sociale (caste), } \\
\text { diététique (puissance } \\
\text { génésique) }\end{array}$ & $\begin{array}{l}\text { Sociale (caste et } \\
\text { classe), diététique } \\
\text { (force), gustative }\end{array}$ & $\begin{array}{l}\text { Sociale (classe), } \\
\text { hygiénique, diététique } \\
\text { (muscle), gustative }\end{array}$ \\
\hline & $\begin{array}{c}\text { Restrictions } \\
\text { pesant sur } \\
\text { la consommation }\end{array}$ & $\begin{array}{l}\text { Culturelle, rituelle, } \\
\text { économique }\end{array}$ & $\begin{array}{l}\text { Culturelle, rituelle, } \\
\text { économique, médicale }\end{array}$ & $\begin{array}{l}\text { Médicale, } \\
\text { éthique, politique, } \\
\text { environnementale? }\end{array}$ \\
\hline & $\begin{array}{l}\text { Classification } \\
\text { des viandes }\end{array}$ & Pur/impur; chaud/froid & $\begin{array}{l}\text { Pur/impur; chaud/ } \\
\text { froid; gras/maigre; } \\
\text { cher/bon marché }\end{array}$ & $\begin{array}{l}\text { Gras/maigre; rouge/ } \\
\text { blanc? }\end{array}$ \\
\hline & $\begin{array}{c}\text { Identification } \\
\text { de l'animal dans la } \\
\text { viande }\end{array}$ & $\begin{array}{l}\text { Recherchée, chair } \\
\text { valorisée }\end{array}$ & Ambivalente & $\begin{array}{l}\text { Rejetée, chair travestie } \\
\text { (protéines, etc.) }\end{array}$ \\
\hline & Statut des abats & Valorisés & $\begin{array}{l}\text { Différenciés } \\
\text { socialement }\end{array}$ & Dévalorisés \\
\hline
\end{tabular}

\section{" Une méthode «tout-terrain»?}

La méthode étant définie très largement, elle est adaptable à de nombreuses situations de terrain. À l'évidence, un premier enjeu est de choisir les lieux où les entretiens et observations seront menés. Le contexte ne permet pas toujours un accès aisé aux lieux qui semblent pourtant les plus idoines pour éclairer les pratiques et les significations relatives à certains aliments. Un site peut être physiquement inaccessible (pour cause d'éloignement par exemple), ou son accès peut être interdit. Il faut être prêt à renoncer à la visite de certains lieux, en cherchant néanmoins à obtenir des informations par des sources secondaires. Dans le cas du terrain indien décrit plus haut, l'accès aux abattoirs municipaux a été plusieurs fois négocié avec les autorités 
locales. Les portes m'ont finalement été ouvertes à condition toutefois de ne pas y prendre de photos. En revanche, il ne m'a pas été possible de faire des observations dans une usine de transformation de produits de volaille. Les fermes avicoles ont quant à elles pu être visitées grâce à des contacts dans la population locale.

Parfois, le parcours de certains aliments est trop complexe pour pouvoir entreprendre des recherches dans tous les lieux traversés. Il faut alors sélectionner ceux pour lesquels on pose l'hypothèse qu'ils contribuent plus que d'autres à déterminer les significations finales qui seront attribuées à l'aliment concerné. Ainsi, en lien avec les limites exposées plus haut, un produit comme des légumes vendus en circuit court ne sera pas étudié de la même façon qu'une bouteille de soda.

Quoi qu'il en soit, l'enquêteur appliquant cette méthodologie doit être ouvert à une forme de sérendipité : il faut se laisser guider par les personnes rencontrées, qui peuvent attirer l'attention ou donner l'accès à des lieux qui n'avaient pas été envisagés jusque-là comme dignes d'intérêt. Il est illusoire de penser pouvoir accorder la même importance à tous les lieux. Pour des raisons épistémologiques, mais aussi pratiques, certains contextes peuvent faire l'objet d'une enquête plus approfondie, laquelle peut alors se rapprocher de l'enquête ethnographique de longue durée. Les autres lieux sont alors visités secondairement et leur étude vient compléter et éclairer les données recueillies dans le principal lieu d'enquête. Dans le travail sur la viande en Inde, les abattoirs et les boucheries ont constitué les principaux lieux d'investigation, car il m'a semblé que c'était là que se jouaient en grande partie les conflits autour des statuts accordés aux différentes viandes. Les pratiques d'élevage ont été étudiées plus secondairement, et toujours en lien avec la question des significations accordées aux différentes viandes.

Rien de surprenant au demeurant à ce qu'une méthode qui s'intéresse à l'effet du contexte sociospatial sur les aliments accepte d'être elle-même façonnée par les différents contextes d'enquête.

\section{"Références}

Appadurai A., 1986. Introduction: Commodities and the Politics of Value, in Appadurai A. (éd.), The Social Life of Things: Commodities in Cultural Perspective, Cambridge, Cambridge University Press, 3-63.

Bell D., Valentine G., 1997. Consuming Geographies: We Are Where We Eat, Londres, New York, Routledge, $248 \mathrm{p}$.

Bruckert M., 2018. La chair, les hommes et les dieux. La viande en Inde, Paris, CNRS éditions, 408 p. Bruckert M., 2021. Chicken Politics: Agrifood Capitalism, Anxious Bodies, and the New Meanings of Chicken Meat in India. Gastronomica: The Journal of Critical Food Studies, 21 (2), 33-46.

https://doi.org/10.1525/gfc.2021.21.2.33

Coles B., 2013. Ingesting Places: Embodied Geographies of Coffee, in Abbots E.-J., Lavis A. (éd.), Why We Eat, How We Eat: Contemporary Encounters between Foods and Bodies, Farnham, Burlington, Ashgate Publishing, 255-270. https://doi.org/10.4324/9781315547190

Cook I., 2004. Follow the Thing: Papaya. Antipode, 36 (4), 642-664.

https://doi.org/10.1111/j.1467-8330.2004.00441.x.

Cook I. et al., 2006. Geographies of food: Following. Progress in Human Geography, 30 (5), 655-666. [En ligne] https://journals.sagepub.com/doi/pdf/10.1177/0309132506070183 (dernière consultation le $28 / 08 / 2021)$. 
Cook I., Crang P., 1996. The World on a Plate. Culinary Culture, Displacement and Geographical Knowledges.Journal of Material Culture, 1 (2), 131-153. https://doi.org/10.1177/135918359600100201

Cook I., Crang P., Thorpe M., 1998. Biographies and geographies: consumer understandings of the origins of foods. British Food Journal, 100 (3), 162-167. https://doi.org/10.1108/00070709810207522 Freidberg S., 2004. French Beans and Food Scares: Culture and Commerce in an Anxious Age, New York, Oxford University Press, 282 p.

Fumey G., 2010. Manger local, manger global. L'alimentation géographique, Paris, CNRS Éditions, $160 \mathrm{p}$.

Goss J., 2004. Geography of Consumption I. Progress in Human Geography, 28 (3), 369-380. https://doi.org/10.1191/0309132504ph486pr

Hassoun J.-P., 2020. Les choses matérielles (les objets, les nourritures) ont-elles un «statut social» qui en quelque sorte les dépasse ? Revue française de science politique, 70 (1), 129-133.

https://doi.org/10.3917/rfsp.701.0121

Hulme A., 2017. Following the (Unfollowable) Thing: Methodological Considerations in the Era of High Globalisation. Cultural Geographies, 24(1), 157-160. https://oi.org/10.1177/1474474016647370

Jackson P., 1999. Commodity Cultures: The Traffic in Things. Transactions of the Institute of British Geographers, 24 (1), 95-108. http://www.jstor.org/stable/623343 (dernière consultation le 28/08/2021).

Latour B., 1996. On actor-network theory: A few clarifications. Soziale Welt, 47 (4), p. 369-381. [En ligne] www.jstor.org/stable/40878163 (dernière consultation le 28/08/2021).

Marcus G., 1995. Ethnography in/of the World System: The Emergence of Multi-Sited Ethnography. Annual Review of Anthropology, 24 (1), 95-117. https://doi.org/10.1146/annurev.an.24.100195.000523 Marx K., 1993. Le capital, livre 1, Paris, PUF, 940 p. (coll. Quadrige).

Mintz S., 1985. Sweetness and Power: The Place of Sugar in Modern History, New York, Viking, 274 p.

Robbins P., 1999. Meat Matters. Cultural Politics along the Commodity Chain in India. Cultural Geographies, 6 (4), 399-423. https://doi.org/10.1177/096746089900600402 


\title{
Chapitre 11 \\ Les enquêtes sociologiques auprès des jeunes mangeurs : enjeux méthodologiques et épistémologiques
}

\author{
AnNe Dupura et Géraldine Comoretto ${ }^{b}$
}

${ }^{a}$ Université de Toulouse Jean Jaurès - ISTHIA, CERTOP - CNRS, France.

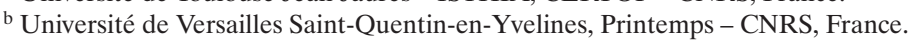

Comme l'alimentation, l'enfance est un objet dont la sociologie s'est emparée tardivement. Le regard porté sur les socialisations est riche d'enseignement pour comprendre la construction sociale des normes et pratiques alimentaires. Ce chapitre présente les enjeux spécifiques à l'enquête sociologique quantitative et qualitative auprès de jeunes mangeurs. Des cas d'applications tirés de recherches des autrices illustrent le propos.

Penser épistémologiquement et méthodologiquement l'enfance, les enfants, en sociologie, n'a pas toujours été une évidence. Le statut spécifique de l'enfance conduit-il à repenser les catégories conceptuelles classiques et les types d'investigation méthodologique de la sociologie? Cette question préalable a guidé la construction de ce chapitre.

Avec le développement, depuis une vingtaine d'années, d'un encadrement normatif de l'alimentation axé sur la médicalisation ${ }^{53}$, la recherche a de plus en plus porté son regard sur divers publics ciblés par les programmes. Parmi eux, les enfants et les adolescents. Ces fronts de recherche ont contribué au développement du dialogue entre les disciplines. Si l'enfance a été l'objet par excellence de la psychologie, c'est de l'anthropologie que provient l'essentiel des appareillages méthodologiques qualitatifs utilisés par les sociologues sur le terrain. Létude des pratiques et consommations alimentaires enfantines ne fait pas appel à une méthode d'enquête à proprement parler, mais plutôt à une palette de méthodologies, dont il serait difficile de rendre compte ici. Le point de vue défendu dans ce chapitre consiste à se saisir

53. Nous pensons notamment au PNNS (programme national nutrition santé) (2001-2005, 2006-2010, 2011-2015, 2019-2023) et au Guide Nutrition des enfants et des adolescents pour tous les parents (2004, réédité en 2019). 
de la spécificité enfantine en méthodologie d'enquêtes alimentaires, précisément parce qu'elle pose un certain nombre de questions de recherche aux sociologues et de défis dans la conception des méthodes.

La première partie présente une synthèse des débats relatifs à la place des enfants dans les enquêtes pour en pointer les enjeux épistémologiques et méthodologiques. Cette partie rend compte du dépassement de l'invisibilité enfantine dans les travaux sociologiques pour en fixer quelques cadres théoriques issus de la sociologie de l'enfance et de la socialisation. Elle interroge également ce que le contexte favorable à la montée de l'éthique de la recherche et de la protection des données personnelles fait à ces débats, en insistant sur la formalisation des données et la standardisation déontologique que ce contexte produit. Enfin, elle évoque ce que la sociologie, ses postures épistémologiques et méthodologiques, peut apporter aux disciplines connexes.

La seconde partie s'intéresse à la pratique méthodologique permettant de saisir le(s) point(s) de vue de l'enfant. Deux exemples tirés de terrains de recherche issus de nos travaux sont proposés. Le premier, depuis la sociologie de l'alimentation, montre comment il est possible d'adapter les études quantitatives du fait alimentaire qui sont conduites auprès des adultes pour pallier l'invisibilité statistique des enfants. Le second, depuis la sociologie de l'enfance, est une ethnographie au long cours inspirée de l'anthropologie, sur les repas et petits repas scolaires, mêlée à des entretiens entre pairs, et entre enfants et parents.

\section{H La prise en compte du point de vue des enfants en débat}

\section{Enfance et alimentation : genèse de deux «petits objets» de la sociologie}

L'enfance partage avec l'objet alimentation une imbrication du biologique, du psychologique et du sociologique mettant à mal l'autonomie du social chère au fondateur de la sociologie en France, Émile Durkheim, héritage expliquant les difficultés à y porter un regard sociologique.

En France, la structuration de deux champs de recherche sociologique sur l'alimentation et l'enfance ne se produit pas sans débat (Poulain, 2017; Sirota, 2019; Lahire, 2019), tandis que, sur la scène européenne et internationale, les dissensions sont moins vives. L'émergence de la sociologie de l'enfance comme celle de la sociologie de l'alimentation s'enracine dans deux mouvements distincts et complémentaires. Le premier correspond à une tradition anglo-saxonne, les childhood studies et les food studies, qui privilégient des approches pluridisciplinaires centrées sur l'objet. Le second s'inscrit dans une tradition française, ancrée disciplinairement, qui favorise les tensions paradigmatiques sur ces deux objets à l'intérieur de la sociologie.

Entre ces deux mouvements, les débats opposent schématiquement, d'une part, des recherches préoccupées de dialogue entre les disciplines (par exemple, pour l'alimentation, entre la nutrition, l'épidémiologie et le monde médical; pour l'enfance, entre la psychologie et la pédiatrie); et, d'autre part, des recherches qui tentent 
d'appréhender les points de vue de, sur et par l'enfant/l'alimentation, en considérant l'objet étudié à la fois comme un lieu de lecture d'un monde social dans lequel il s'insère et comme un lieu où il est possible d'appréhender le social en construction.

Si ces deux mouvements sont en tension, l'accès de l'enfance et de l'alimentation au statut d'objets sociologiques légitimes depuis une vingtaine d'années favorise aujourd'hui des postures d'échange dépassant ces clivages.

\section{Une pluralité de dispositifs pour investiguer l'alimentation enfantine}

Les appareillages méthodologiques pour étudier spécifiquement les publics enfantins s'avèrent d'une grande diversité. La pratique sociologique, plus ouverte que par le passé, est moins attachée à des postures paradigmatiques et aux techniques d'enquêtes privilégiées par celles-ci. Les sociologues manient plus volontiers qu'auparavant un grand nombre d'outils. Ils participent ainsi à produire un point de vue des/sur les enfants/l'alimentation depuis la mobilisation d'approches qualitatives largement répandues dans les usages. Ils permettent ainsi de décrire des pratiques et d'analyser le sens qui leur est donné selon leurs contextes de production comme à maintenir l'invisibilisation statistique des enfants et de leurs points de vue, y compris dans les enquêtes alimentaires, les parents étant interrogés pour répondre aux questions sur l'alimentation de leur enfant. Si l'intérêt pour les pratiques quotidiennes en contexte va croissant, il autorise l'émergence de cette troisième voie, laquelle favorise le dialogue entre les approches à l'intérieur de la sociologie et entre les disciplines. Elle met au jour les articulations possibles entre capacités d'agir et déterminismes sociaux, différenciations sociales et pensée développementale suivant différents stades.

Le choix de la méthodologie de recherche doit s'appuyer sur la définition explicite de ce qu'est un enfant, en tenant compte de son âge, de son statut d'enfant, des modèles d'enfance et de la manière dont ces différents aspects sont construits socialement dans le contexte de l'étude. Deux références méthodologiques s'imposent ici. La première présente les points nécessaires à appréhender pour concevoir un appareillage méthodologique en adoptant une focale infanto-centrée (Danic et al., 2006). Grâce à une réflexion sur la construction des âges de l'enfant et sur la relation enquêté/enquêteur, cet ouvrage permet de dépasser les impensés de la recherche et les stéréotypes qui leur sont parfois associés (par exemple, l'enfant perroquet, l'enfant naïf, l'enfant immature, l'enfant menteur). Cet ouvrage souligne comment chaque chercheur travaillant sur cette population doit s'attacher à définir ce que signifie l'enfance. Ce faisant, il contribue à la réflexion scientifique sur les découpages des âges de la vie. Le déni des modèles d'enfance construits historiquement, le primat d'un modèle abstrait, universel, ethnocentré de l'enfant, pensé depuis la psychologie et donnant l'illusion d'une certaine homogénéité de l'enfance, les âges, pensés comme des réalités naturelles et non façonnées socialement et culturellement, sont autant d'ouvertures réflexives apportées par cet ouvrage.

Une seconde référence propose des dispositifs concrets pour la mise en œuvre d'enquêtes alimentaires chez les enfants en s'appuyant sur le registre ludique auquel les auteurs les associent (Lalanne et Tibère, 2012). S'inspirant davantage du format «guide méthodologique» par l'introduction de conseils puisés dans 
les méthodes visuelles (dessins, images) et ludiques (jeux), cet article permet une entrée concrète et stimulante sur une conception ludique de la relation aux enfants dans la pratique du terrain.

\section{La place des enfants selon les contextes}

Sonder la place des enfants en contextes, c'est questionner l'engagement du sociologue dans le travail de terrain, plus particulièrement ici, en relation avec les enquêtés. Enquêter auprès des enfants c'est, dans le contexte occidental, se heurter à des problèmes déontologiques relatifs au recueil de la parole d'enfants mineurs qui implique des consentements éclairés des enfants et des adultes formalisés par des autorisations parentales voire institutionnelles (écoles ou associations sportives par exemple, voire institutions de tutelle pour les enfants placés à l'assistance publique). Ce contexte de recueil de données oriente plutôt les recherches vers le milieu familial, les autres milieux sociaux étant des terrains plus compliqués à négocier pour le chercheur. Cependant, comme nous le verrons, les terrains extra-familiaux, plus particulièrement dans le contexte scolaire, sont envisageables.

Au cours de la collecte, la présence d'au moins un adulte à proximité ou la mise en œuvre de dispositifs permettant de recueillir la parole et les gestes des enfants (enquête dans des espaces ouverts, vitrés et non clos) facilitent l'accès au terrain dans le respect des règles de déontologie de la pratique sociologique, même si le chercheur doit être attentif à ce que l'éducateur et les effets que sa présence produit sur les échanges ne prennent pas le dessus. Dans la pratique sociologique il s'agit également d'instaurer la confiance auprès des parents (qui peuvent craindre le jugement du chercheur sur leurs pratiques éducatives) et auprès des enfants pour que ces derniers comprennent pourquoi l'adulte vient apprendre d'eux (ce qui s'apparente à une inversion de la relation verticale au savoir et de dépendance avec les adultes).

Léthique de la recherche est liée aux conditions de réalisation des enquêtes comme aux interactions que les chercheurs nouent avec les personnes étudiées. Ces questions d'éthique sont aujourd'hui devenues incontournables, y compris en sciences sociales. Un règlement européen sur la protection des données personnelles (RGPD) régule désormais la conformité juridique et potentiellement éthique des recherches; les grandes institutions de recherche se sont dotées de comités éthiques et les revues exigent de plus en plus souvent des preuves de conformité. Cette mise en conformité des protocoles de collecte implique de définir en amont les méthodes de recueil de la parole des enfants et de l'observation de leurs pratiques, pour les faire valider. Cette évaluation participe ainsi, incidemment, à privilégier des formats hypothéticodéductifs au détriment des approches inductives ou inspirées de la grounded-theory. Ainsi, la production scientifique s'en trouve transformée. En obligeant les chercheurs à énoncer l'ensemble des critères, domaines et variables étudiées, ce cadrage facilite néanmoins, selon nous, l'imposition d'une troisième voie dans les recherches en alimentation chez l'enfant. Il pousse les chercheurs à anticiper les conditions d'un dialogue possible ou les réponses aux exigences des évaluateurs (des projets ou articles soumis) dont la discipline ou la visée paradigmatique peut varier de la leur, plutôt qu'à s'y adapter a posteriori. 


\section{Apports de la méthode pour les disciplines connexes}

Questionner les apports de la méthode pour les disciplines connexes revient ici à discuter des apports d'une discipline, la sociologie. «Pour définir la sociologie, il convient de partir à la fois de son objet et de sa pratique. Son objet renvoie à l'homme social ou à l'homme socialisé. [...] Il ne peut y avoir de savoir sociologique indépendamment des techniques mises en œuvre pour y accéder. Autrement dit, le sociologue ne se contente pas d'avoir des idées sur la société. Il réalise des enquêtes et entend mettre les théories générales à l'épreuve de la réalité empirique qu'il observe et interprète. Ses conclusions résultent d'un processus maîtrisé d'administration de la preuve (Paugam, 2012, p. 1-2).

La rigueur scientifique, l'engagement sur le terrain et la visée particulièrement critique et réflexive qui construisent l'expérience du sociologue dans ses enquêtes sont des apports fondamentaux de la discipline pour les domaines connexes. Le débat engagé par André Turmel en sociologie de l'enfance avec la psychologie du développement illustre parfaitement cet apport concernant la normalisation de l'enfance. Cette dernière résulte des mesures physiques et psychiques imposées aux enfants, lesquelles sont pensées en termes maturationnistes, et fixent des étapes de développement contribuant à organiser socialement la croissance, l'avancée en âge et le corps en transformation (Turmel, 2008). Appliqué à l'alimentation, cet apport a été remobilisé pour dialoguer, avec les psychologues du goût et les nutritionnistes, de la notion de néophobie alimentaire, largement répandue dans ces disciplines, laquelle est pensée en processus maturationniste, développemental et universel (Dupuy et al., 2021).

\section{- La place des enfants en pratique}

Les techniques d'enquête sociologique permettent d'étudier les comportements alimentaires des enfants. Nous présenterons deux exemples d'enquêtes au sein de deux instances de socialisation primaire différentes : le milieu familial et le milieu scolaire. Chaque enquête combine plusieurs méthodes d'investigation auprès des jeunes mangeurs.

\section{L'enquête statistique}

Accéder au point de vue des enfants tout en prenant en considération l'influence de leur environnement implique de combiner plusieurs méthodes d'enquête. Ce premier exemple est celui d'une recherche sur la place du plaisir dans la socialisation des enfants et des adolescents (Dupuy, 2013).

Des entretiens individuels, des discussions de groupes, des observations ont été menés dans des familles et des écoles dans l'objectif de combiner les points de vue et de révéler les processus de différenciations sociales dans la construction des dispositions au plaisir alimentaire au sein de la famille et parmi les amis. Cette approche, inspirée du travail du sociologue Bernard Lahire, a permis de mettre au jour la diversité des dispositions au plaisir, leurs origines sociales et la manière dont ces dispositions pouvaient être actualisées ou inhibées selon les contextes sociaux. Pour croiser les points de vue, des entretiens collectifs avec plusieurs membres d'une même fratrie ou d'un même groupe de pairs, ainsi que d'autres réunissant des 
enfants, leurs parents et grands-parents ont également été réalisés. Simultanément, une enquête quantitative a été conduite auprès de 2528 individus, dont 1002 âgés de 7 à 17 ans. Cette enquête par questionnaire est originale en ce qu'elle a consisté à interroger directement des enfants et adolescents, contrairement aux usages classiques des enquêtes d'épidémiologie nutritionnelle, qui interroge généralement les parents au sujet de l'alimentation de leurs enfants ${ }^{54}$.

\section{Spécificités de l'enquête par questionnaire auprès de jeunes mangeurs}

Cette enquête quantitative avait des particularités. Tout d'abord, les durées des questionnaires ont été conçues différemment pour les enfants (30 minutes) et les adultes (40 minutes). Ensuite, l'enquête a été construite de manière à garder la trace des «unités familiales» des jeunes mangeurs $(n=1002)$ et de leurs parents $(n=624)$, eux aussi questionnés. La population enfantine interrogée était entre autres composée de 374 fratries. Les questions ont été posées par des enquêteurs au sein des foyers, en interrogeant séparément enfant(s) et parent(s), comme frères et sœurs. Enfin, elle a été réalisée en face-à-face, au domicile des enquêtés, dans cinq grandes régions de la France métropolitaine.

L'interrogation en face-à-face et au domicile des enquêtés est probablement la méthode la plus fiable et la plus riche car elle offre la possibilité de creuser certaines questions sur le mode de l'entretien semi-directif (e.g. la reconstruction des journées alimentaires avec des outils d'anamnèse) et parce qu'elle rend envisageable des questionnements plus complexes. Cependant, ce type de dispositif est très coûteux, du fait du déplacement in situ de nombreux enquêteurs sur un large territoire. Il est aussi chronophage, car il s'appuie sur des questionnaires différenciés et implique une importante formation des enquêteurs : les manuels d'enquête et les sessions de formation sont impératifs (annexe 1). Cette préparation permet aux enquêteurs de s'approprier les objectifs des questions, de les adapter au jeune public par un travail de traduction, le recours au tutoiement et de comprendre la nécessité de ne pas imposer son point de vue. Pour les enquêteurs, la complexité assez inhabituelle de cette enquête, ainsi que sa finalité «recherche» ont été globalement perçues comme gratifiantes, valorisant leur rôle. La formation des enquêteurs comportait deux points centraux : la mise en garde contre toute forme de jugement de valeur face aux réponses apportées; la préparation à ne pas anticiper et à ne pas proposer de réponses dans les questions ouvertes. Face à des enfants, dans une relation adulte-enfant forcément dissymétrique, il leur était ainsi demandé de plutôt accompagner les réponses, parfois hésitantes, en s'appuyant sur des procédés habituels dans les techniques d'entretiens semi-directifs telles que les relances «mot à mot» des propos des enfants.

\section{Le principe de standardisation à l'œuvre dans l'enquête par questionnaire}

Le principe de standardisation est au fondement de cette méthodologie comparative et causale, laquelle vise à restituer des différences sociales selon l'âge, le sexe

54. Ce sont le cas par exemple pour les enquêtes Inca et Nutrinet. 
ou encore la position sociale. En général, ce principe prend effet sur la forme du questionnaire (homogénéisation des conditions de passation, consignes, indications, recommandations, ordres des items) comme sur le fond (types de questions et modalités de réponses). Cette exigence de standardisation pose problème lorsqu'on s'adresse à un public aussi hétérogène que des enfants et des adultes, le questionnaire des enfants devant répondre à une exigence de similarité pour comparer les réponses données par des enquêtés différents tout en étant adapté à certaines particularités mentionnées précédemment.

Ce travail d'homogénéisation des questionnaires ayant pour objectif de favoriser des comparaisons inter- et intragénérationnelles sur tous les items (les enfants avec leur parent; les enfants entre eux dans le cadre des fratries), ainsi que des comparaisons dynamiques sur les comportements alimentaires (ce corpus ajouté aux données des enquêtes antérieures menées par Poulain, 2001), la standardisation a été opérée à deux niveaux :

- en général avec l'adoption d'une posture infanto-centrée pour les formulations des trois questionnaires;

- et plus spécifiquement, pour le questionnaire «enfant», avec la suppression de questions lorsqu'elles étaient non comprises ou pour répondre à un impératif (scientifique concernant l'attention, et économique) de réduction dans la durée de passation, puis avec l'usage du tutoiement par l'enquêteur dans la formulation des questions (annexe 1).

Pour se conformer à ce principe de standardisation, le travail de construction du questionnaire s'est effectué par le repérage et la sélection de questions dans des enquêtes antérieures relatives aux comportements alimentaires d'adultes ${ }^{55}$ ou aux styles éducatifs dans l'alimentation qu'il a fallu transposer en des questions compréhensibles pour des enfants, ainsi que par l'élaboration de questions originales relatives au plaisir alimentaire. Ce travail a été possible dans sa correspondance étroite avec l'enquête qualitative et des phases de tests des diverses versions du questionnaire auprès des publics concernés, notamment pour les choix des mots afin de limiter les éventuelles différences d'interprétation des questions et des réponses. Cette phase de test a permis d'abandonner l'adossement des questions relatives à la composition des repas à une banque de dessins d'items (entrée, plat garni, dessert par exemple) prévus initialement dans le dispositif de collecte, laquelle générait des effets d'imposition dans les réponses des enfants.

\section{L'enquête ethnographique}

Ce second exemple d'enquête auprès de jeunes publics est celui d'une recherche menée sur les aspects sociaux des repas entre enfants à l'école élémentaire (Comoretto, 2015), qui s'appuyait sur un dispositif méthodologique complexe empruntant des outils à la sociologie, l'ethnologie et l'anthropologie.

L'enquête s'est déroulée sur deux années scolaires dans trois écoles élémentaires de la région parisienne. Les établissements scolaires ont été choisis de façon à varier les

55. Ont donc été collectées sur ce même modèle (Poulain, 2001) des données de représentations, d'opinions, de comportements déclarés ou de comportements reconstruits. Des questions spécifiques à notre recherche se sont ajoutées, sur le thème du plaisir et sur celui des styles éducatifs. 
milieux sociaux. Des entretiens semi-directifs ont été conduits au domicile familial auprès de parents. Les surveillants, animateurs socioculturels et agents de restauration qui encadrent les élèves lors des repas ont également été interrogés. Mais la partie du protocole méthodologique qui nous intéresse dans ce chapitre est celle des investigations menées exclusivement avec et auprès des enfants, dans le cadre périscolaire des repas à la cantine et du goûter à l'étude du soir. L'enjeu est ici de mettre en lumière ce choix de placer les enfants au cœur du dispositif et de les faire participer de manière active à l'enquête.

La particularité de cette recherche tient au fait que nous souhaitions étudier les pratiques alimentaires des enfants à l'école sans pour autant nous intéresser directement à ce qu'ils mangent. Nos observations reposaient sur le caractère social des prises alimentaires en cadre scolaire et ce qu'elles nous apprennent de la transmission de dispositions sociales et du rôle des pairs dans la construction sociale des enfants.

Pour observer les sociabilités enfantines au prisme des repas à l'école, le choix de réaliser une enquête qualitative, résolument inscrite dans une démarche ethnographique et empirique, s'est vite imposé. Comment envisager autrement que par la présence récurrente du chercheur sur le terrain l'observation des pratiques alimentaires des élèves, répartis par tablées dans la cantine ou par petits groupes dans la cour de récréation et évoluant au rythme des amitiés et des inimitiés enfantines? Nous nous sommes immergée dans ce quotidien enfantin en nous efforçant de nous rendre le plus souvent et le plus régulièrement possible dans les écoles lors des repas.

Deux postures ont été adoptées pour nous approcher au plus près des interactions enfantines. La première, inspirée de ce que William Corsaro (1997) nomme «reactive method», consiste en un maintien à distance de l'enquêtrice lors des premiers jours d'observation, à la cantine comme lors du goûter, afin d'offrir la possibilité aux enfants d'être seuls décideurs du moment auquel ils allaient (ou non) entrer en contact avec l'observateur. La seconde approche renvoie à la notion de «least-adult child» développée par Nancy Mandell (1988). C'est en passant du temps auprès des enfants, en partageant leurs activités régulièrement et en leur accordant de l'intérêt que les diverses barrières qui séparent les enfants et l'adulte pourront s'abaisser. C'est en se montrant le «moins adulte possible», en adoptant les pratiques des enfants sans essayer ni de les diriger, ni de les contrôler, que le chercheur parvient à pénétrer pleinement l'univers enfantin. Pour notre part, nous avons fait tantôt le choix de l'observation à distance, tantôt celui de l'observation participante, en respectant néanmoins les limites imposées par les encadrants (en ne jouant pas avec les aliments à la cantine par exemple).

\section{Les observations à la cantine}

Que l'on observe à distance ou que l'on opte pour une observation participante, la cantine scolaire constitue un terrain de recherche difficile à appréhender tant il est dense en termes d'interactions. Nous avons réalisé 200 heures d'observations dans les restaurants scolaires et partagé 65 repas à table avec les élèves. Pendant une partie du service, nous restions mobile dans le restaurant scolaire afin de varier les postes d'observation : d'une salle à l'autre, d'une table à l'autre, d'une interaction à l'autre. Pendant cette heure d'observation, nous prenions des notes 
dans notre journal de terrain tout en maintenant nos échanges avec les enfants et le personnel de la restauration scolaire. Il nous semblait difficile d'appliquer une grille d'observation stricte, dans la mesure où il n'est pas un jour où il se passe la même chose que la veille ou le lendemain, mais aussi car cela aurait impliqué de restreindre la focale sur un groupe d'acteurs ou un type de pratiques. Malgré tout, nous nous sommes attachée à dégager des constantes dans notre prise de notes dès les premiers jours d'observation tel que le placement à table des élèves selon leur âge et leur niveau (tableau 11.1).

Tableau 11.1. Exemple de recension du placement à table $(\mathrm{T})$ des élèves à la cantine.

\begin{tabular}{|c|c|c|c|c|c|c|c|}
\hline \multicolumn{8}{|c|}{$\mathbf{1}^{\mathrm{er}}$ service } \\
\hline & Filles & Garçons & & & Filles & Garçons & \\
\hline T1 & 9 & 0 & + animatrice & T8 & 5 & 4 & + animatrice \\
\hline $\mathbf{T} 2$ & 3 & 6 & + animatrice & T9 & 2 & 8 & \\
\hline $\mathbf{T 3}$ & 3 & 6 & & T10 & 0 & 0 & \\
\hline T4 & 5 & 4 & + enquêtrice & T11 & 0 & 0 & \\
\hline T5 & 0 & 0 & & T12 & 7 & 1 & \\
\hline T6 & 3 & 6 & & T13 & 4 & 6 & \\
\hline T7 & 0 & 0 & & T14 & 4 & 4 & + animatrice \\
\hline & & Filles $=45$ & & Garç & $s=45$ & & Total $=90$ \\
\hline
\end{tabular}

Dans les écoles proposant un service à table, nous passions la deuxième heure à table avec les enfants, nous partagions le même repas qu'eux, passant ainsi 45 minutes à table avec un groupe restreint d'élèves. Ce rôle plus participatif implique un travail de mémorisation, puis de retranscription des observations puisqu'il contraint le chercheur à délaisser, le temps du repas, son journal de terrain afin de prendre pleinement part aux interactions de table avec les enfants. Une fois le service fini, nous prenions soin de retranscrire au plus vite les informations recueillies.

\section{Les échanges de goûter à l'étude}

L'observation d'une prise alimentaire comme le goûter à l'étude constitue un défi ethnographique pour le chercheur. En effet, les goûters se déroulent lors de l'étude surveillée, où les écoliers n'ont de contraintes physiques que celles qu'ils s'imposent, contrairement à ce qui se passe lors du déjeuner à la cantine. La prise alimentaire se déroulant le plus souvent dans la cour de récréation, les élèves peuvent décider de prendre leur goûter où ils le souhaitent et comme ils le souhaitent : assis, en marchant, en courant, en jouant. Ainsi, suivre entre cinquante et cent enfants en une demi-heure dans le vaste espace d'une cour de récréation nécessite une certaine rigueur méthodologique.

$\mathrm{Si}$, lors des premières séances, nous nous sommes contentée d'observer la façon dont se déroulait la prise alimentaire en nous déplaçant au hasard dans la cour de récréation, il nous a rapidement fallu définir plus précisément ce sur quoi nous souhaitions enquêter - les transactions de goûter - et mettre en place une méthode minutieuse 
de collecte des données. Les recensions de goûter ont consisté à repérer les élèves présents le plus régulièrement à l'étude, soit trois ou quatre fois par semaine, afin de noter, lors de chaque séance d'observation, la composition exacte de leur goûter après échanges (tableau 11.2).

Tableau 11.2 Exemple de recension de goûter.

\begin{tabular}{|c|c|}
\hline & Grille des goûters recensés de Clothilde, CM2, école de milieu supérieur \\
\hline Jour 6 & 1 pomme - 2 Savane \\
\hline Jour 7 & 1 pomme -2 Prince \\
\hline Jour 10 & 1 Pom'potes bio -5 mini-BN chocolat \\
\hline Jour 11 & $\begin{array}{l}1 \text { Pom'potes bio }-1 \text { barre chocolatée de Madeleine } \\
1 \text { mini-tartelette fraise }-1 / 2 \text { mini-cake fraise de Léo }\end{array}$ \\
\hline Jour 12 & $\begin{array}{l}2 \text { prunes }-6 \text { mini-BN chocolat }-1 \text { faux Petit écolier - } 1 / 2 \text { Pépito brownito de Léo } \\
1 \text { Petit Couur de Mathilde }\end{array}$ \\
\hline Jour 13 & 2 prunes -5 mini-BN chocolat $-1 / 2$ Pépito de Madeleine \\
\hline Jour 14 & $\begin{array}{l}\text { Pain maison farine bio }+ \text { Nutella } \\
2 \text { carrés de Kinder Bueno white de Victorien } \\
1 \text { beignet nature de Madeleine }\end{array}$ \\
\hline Jour 15 & 1 Pom'potes bio - Pain bio + Nutella bio -1 gâteau à la fraise bio \\
\hline Jour 16 & $\begin{array}{l}1 \text { Pom'potes bio }-3 \text { tranches de pain de mie bio }+ \text { nutella bio } \\
1 / 2 \text { Prince chocolat d'Audrey }-1 \text { Carambar de Madeleine } \\
1 \text { quille de Mammouth de Madeleine (reprise dans la poubelle) }\end{array}$ \\
\hline Jour 17 & $\begin{array}{l}1 \text { Pom'potes bio } \\
3 \text { tranches de pain de mie complet bio sans croûte }+ \text { pâte à tartiner bio } \\
3 \text { bâtonnets }+ \text { Nutella de Madeleine }\end{array}$ \\
\hline Jour 18 & 1 clémentine bio -3 crêpes bio -1 bonbon d'Oriane \\
\hline Jour 19 & 1 Pom'potes bio -1 morceau de baguette bio -6 mini-BN chocolat \\
\hline Jour 20 & Raisin noir bio -6 mini-BN chocolat \\
\hline Jour 21 & $\begin{array}{l}\text { Pain fait maison }-1 \text { Kinder surprise }-1 \text { crêpe Whaou chocolat } \\
1 \text { pomme (non mangée) }\end{array}$ \\
\hline Jour 22 & $\begin{array}{l}1 \text { Pom'potes bio - Pain fait maison }-1 \text { Kinder surprise } \\
1 \text { Napolitain de Madeleine }-1 \text { bonbon de Madeleine }\end{array}$ \\
\hline Jour 23 & 1 Pom'potes bio -1 crêpe Whaou chocolat -2 mini-BN chocolat \\
\hline Jour 24 & 1 Pom'potes bio - 1 crêpe Whaou chocolat \\
\hline
\end{tabular}

Les prénoms des enfants cités dans le tableau sont des camarades de Clothilde ayant partagé avec elle une partie de leur propre goûter. Ces données qualitatives ont ensuite fait l'objet d'un traitement statistique afin, d'une part, de qualifier et de quantifier la composition des goûters selon l'origine sociale des élèves; d'autre part, d'analyser les transactions non marchandes opérées dans la cour de récréation et de représenter graphiquement les réseaux de transactions. Ce passage d'un matériau qualitatif recueilli par observation à un usage quantitatif a suscité quelques obstacles lors du traitement et de l'analyse des données. La principale difficulté réside dans l'instabilité des bases de données. Bien que nous ayons pris le soin de 
sélectionner un échantillon d'élèves à suivre prioritairement, tous n'étaient pas systématiquement présents : nous obtenons donc des taux de présence lors des recensions très variables d'un élève à l'autre. Il nous a fallu tenir compte de ce biais dans l'analyse des transactions de goûters.

\section{Une méthodologie adaptée aux enfants}

Enquêter sur l'alimentation des enfants en cadre scolaire nécessite donc de définir comment observer, participer, retranscrire, compter et analyser les comportements enfantins autour de l'acte alimentaire. Il s'agit également d'instaurer des méthodes d'enquête adaptées au jeune public lorsque le terrain l'impose. L'approche ethnographique permet à la commensalité et à la convivialité qui émanent du partage des repas avec les élèves d'abaisser les frontières entre l'adulte et les enfants, et de s'approcher aux plus près des sociabilités enfantines.

Selon les écoles et les interlocuteurs, nous avons endossé une pluralité de rôles aux yeux des enquêtés. Les élèves nous ont tantôt perçue comme une étudiante, une inspectrice, une animatrice ou une «dame de cantine». Si le statut de chercheur peut parler à certains enfants, celui de sociologue leur est inconnu. En choisissant de laisser les enfants venir vers nous, ils ont pu s'imaginer toutes sortes de scénarios en nous voyant prendre des notes dans notre journal de terrain. Les élèves des trois écoles n'ont pas réagi de la même façon à notre présence : ceux qui se sont le plus rapprochés de l'enquêtrice sont ceux avec qui la distance sociale et culturelle était la moins grande, à savoir les enfants issus des classes moyennes et supérieures. De ce point de vue, enquêter auprès d'enfances diverses suscite les mêmes problématiques pour le chercheur qu'une enquête auprès d'adultes issus de milieux sociaux distincts et nécessite d'adapter la relation d'enquête au public rencontré.

Paradoxalement, après avoir pris le temps de gagner la confiance des enfants, il nous a parfois fallu instaurer des barrières et apprendre à ne pas être trop proche des élèves, à diversifier les relations d'enquête lorsque la facilité nous poussait à aller vers ceux qui étaient le plus en demande d'interaction. Nous avons donc pris garde à ne pas toujours déjeuner avec les mêmes enfants, à demander aux garçons de nous accueillir à leur table et à interagir avec ceux qui se montraient les plus réticents. L'interconnaissance et l'aisance sur un terrain peuvent constituer tant un atout qu'un handicap pour le chercheur s'il n'opère pas un travail de distanciation suffisant, d'autant plus peut-être dans le cadre d'une relation avec de "petits enquêtés », avec lesquels la dimension affective est forte.

Pour conclure ce chapitre, les étapes d'élaboration entreprises pour la collecte de données font partie intégrante du processus de recherche. Malheureusement elles sont trop souvent absentes des écrits finaux qui tendent à faire disparaître les procédés mis en œuvre et les tâtonnements du chercheur au profit d'analyses abouties et lissées dans la restitution finale. Les restituer dans une analyse méthodologique participe pleinement en retour à un travail de mise à distance sur ses propres données et sur la manière dont on se les procure, puisque les cheminements suivis sont rarement en ligne droite. Ils font l'objet d'allers et retours entre la collecte, les lectures, le terrain et les questions principales de recherche. La non-évidence des 
enquêtes alimentaires auprès des enfants, pour toutes les raisons évoquées succinctement dans ce chapitre, invitent (si ce n'est contraignent) les chercheurs à combiner étroitement analyses méthodologiques et analyses sociologiques dans leurs raisonnements scientifiques.

\section{" Références}

Corsaro W., 1997. The Sociology of Childhood, Londres, Sage, 456 p. (coll. Sociology for a New Century Series).

Comoretto G., 2015. Manger entre pairs à l'école. Synchronisme et complémentarité des processus de socialisation, Thèse de doctorat de sociologie, Université de Versailles Saint-Quentin-en-Yvelines, 596 p. [En ligne] https://tel.archives-ouvertes.fr/tel-01486529/document (dernière consultation le 28/08/2021).

Danic I., Delalande J., Rayou P., 2006. Enquêter auprès d'enfants et de jeunes : objets, méthodes et terrains de recherche en sciences sociales, Rennes, Presses universitaires de Rennes, $216 \mathrm{p}$.

Dupuy A., 2013. Plaisirs alimentaires, socialisation des enfants et des adolescents, Rennes, Presses universitaires de Rennes, Presses universitaires François Rabelais de Tours, 475 p. (coll. Tables des hommes).

Dupuy A., Nicklaus S., Schwartz C., Goirand S., Tibère L., 2021. Young Children's Learning about Hunger and Satiety through the Lens of the Norms of Those Who Feed Them. Social Sciences, 10 (8), p. 292. https://doi.org/10.3390/socsci10080292

Lahire B. (éd.), 2019. Enfances de classe. De l'inégalité parmi les enfants, Paris, Seuil, 1232 p.

Lalanne M., Tibère L., 2012. Enquête alimentaire auprès des enfants (méthodologie), in Poulain J.-P. (éd.), Dictionnaire des cultures alimentaires, Paris, Presses universitaires de France, 461-470 (coll. Quadrige).

Mandell N., 1988. The least-adult role in studying children. Journal of Contemporary Ethnography, 16 (4), 463-467. https://doi.org/10.1177/0891241688164002

Paugam S., 2021. Introduction - L'enquête sociologique en vingt leçons, in Paugam S. (éd.), L'enquête sociologique, Paris, Presses universitaires de France, 1-4 (coll. Quadrige).

Poulain J.-P., 2001. Manger aujourd'hui : attitudes, normes et pratiques, Toulouse, Privat, 235 p.

Poulain J.-P., 2017. Socio-anthropologie du «fait alimentaire» ou food Studies. Les deux chemins d'une thématisation scientifique. L'année sociologique, 67 (1), 23-46.

Sirota R., 2019. Positions et dispositions de la sociologie de l'enfance. Retour sur le processus de socialisation, in Depoilly S., Kakpo S., La différenciation sociale des enfants. Enquêter sur et dans les familles, Saint Denis, Presses universitaires de Vincennes, 27-52 (coll. Culture et Société).

Turmel A., 2008. Une sociologie historique de l'enfance. Pensée du développement, catégorisation et visualisation graphique, Laval, Presses de l'université Laval, 366 p. (coll. Sociologie contemporaine). 


\section{Annexe 1 : extrait du Manuel de l'enquêteur pour le questionnaire enfant : la reconstruction de la journée alimentaire de la veille (tiré de Dupuy, 2013)}

Partie 3 : les pratiques alimentaires

La reconstruction des journées alimentaires demande une attention particulière :

il faut aider l'interviewé à se remémorer ses consommations de la veille, en tenant compte du fait qu'il peut en oublier, voire en omettre volontairement si elles lui semblent culpabilisantes.

Des phrases du type «dans l'après-midi, ou dans la soirée, vous n'avez rien pris?» sont efficaces si l'enquêteur montre qu'il ne juge pas (qu'il reste neutre).

Il faut faire attention également à ne pas influencer et parasiter les réponses en gardant une certaine distance et en n'émettant pas d'avis ou d'exemples personnels.

Il faut inscrire ce que dit l'interviewé (en le faisant re-formuler s'il n'est pas clair) et pas une interprétation de ce qu'il dit.

\begin{tabular}{|c|c|c|c|}
\hline $\begin{array}{l}\text { Reconstruction } \\
\text { des journées } \\
\text { alimentaires } \\
\text { D'hier matin } \\
\text { à hier soir }\end{array}$ & Q7 & $\begin{array}{l}\text { Nous allons maintenant nous } \\
\text { intéresser à ce que tu as mangé } \\
\text { durant la journée d'hier (d'hier } \\
\text { matin à hier soir). Je vais te } \\
\text { demander de me décrire tout ce } \\
\text { que tu as bu et mangé depuis ta } \\
\text { première prise d'hier matin jusqu'à } \\
\text { la dernière. }\end{array}$ & 1 tableau par prise \\
\hline $\begin{array}{c}\text { Heure } \\
\text { de la prise }\end{array}$ & & $\begin{array}{l}\text { À quelle heure a eu lieu ta } \\
\text { première prise, solide ou liquide, } \\
\text { de la journée d'hier? }\end{array}$ & $\begin{array}{l}\text { Noter l'heure de la prise } n^{\circ} 1 \text {. S'il s'agit } \\
\text { d'un verre d'eau, laisser parler pour } \\
\text { aider l'enquêté à se repérer, mais ne pas } \\
\text { noter (l'eau n'est pas considérée comme } \\
\text { une prise alimentaire dans le cadre } \\
\text { de cette étude) }\end{array}$ \\
\hline $\begin{array}{c}\text { Contenu } \\
\text { de la prise }\end{array}$ & & De quoi s'agissait-il? Décris-le... & $\begin{array}{l}\text { Noter un aliment par case } \\
\text { Ne pas noter l'eau }\end{array}$ \\
\hline $\begin{array}{l}\text { Définition } \\
\text { par l'interviewé } \\
\text { de cette prise }\end{array}$ & & $\begin{array}{l}\text { Comment appelles-tu cette } \\
\text { consommation? C'était quoi } \\
\text { pour toi? }\end{array}$ & $\begin{array}{l}\text { C'est à l'interviewé de dire s'il s'agissait } \\
\text { d'un petit-déjeuner, d'un déjeuner ou } \\
\text { d'un dîner, ou d'une prise hors repas } \\
\text { Cocher la modalité correspondante... }\end{array}$ \\
\hline $\begin{array}{l}\text { Contexte social } \\
\text { de consommation }\end{array}$ & & $\begin{array}{l}\text { Pour cette consommation, as-tu } \\
\text { mangé seul ou en compagnie } \\
\text { de quelqu'un? Avec qui? }\end{array}$ & $\begin{array}{l}\text { Cocher la modalité correspondante. } \\
\text { Si en compagnie, préciser si enfants } \\
\text { ou/et adultes }\end{array}$ \\
\hline $\begin{array}{l}\text { Lieu de } \\
\text { consommation : } \\
\text { hors domicile }\end{array}$ & & $\begin{array}{l}\text { Où as-tu consommé cet (ces) } \\
\text { aliment(s)? Si l'interviewé a acheté } \\
\text { dans un snack et a mangé en } \\
\text { marchant, sans s'asseoir, cochez } \\
\text { « dans la rue». }\end{array}$ & Cocher la modalité correspondante \\
\hline $\begin{array}{l}\text { Consommations } \\
\text { à domicile }\end{array}$ & & $\begin{array}{l}\text { Pour les consommations faites à } \\
\text { la maison : tu étais debout ou assis } \\
\text { etc.? }\end{array}$ & $\begin{array}{l}\text { Cocher les modalités correspondantes. } \\
\text { Si la personne interrogée est assise et } \\
\text { dans la cuisine, cocher les } 2 \text { modalités. } \\
\text { Identifier les prises qui accompagnent } \\
\text { la préparation du repas } \\
\text { Notez en clair la durée déclarée de } \\
\text { la prise. }\end{array}$ \\
\hline $\begin{array}{l}\text { Prise } n^{0} 2 \\
\text { et ainsi de suite }\end{array}$ & & $\begin{array}{l}\text { Puis passer à la prise suivante en } \\
\text { aidant l'enquêté à se remémorer sa } \\
\text { journée de la veille. Et après, tu te } \\
\text { souviens? }\end{array}$ & Noter dans le tableau 2 \\
\hline
\end{tabular}


Ce dispositif d'enquête s'appuie sur un outil de remémoration des diverses prises alimentaires structurant la journée pour des populations adultes (Poulain, 2001). En passant en revue, pour chaque prise, un ensemble de variables pour assister l'anamnèse, ce procédé d'investigation permet d'obtenir des pratiques alimentaires reconstruites a posteriori plus précises que les pratiques déclarées sans l'assistance de l'enquêteur. 


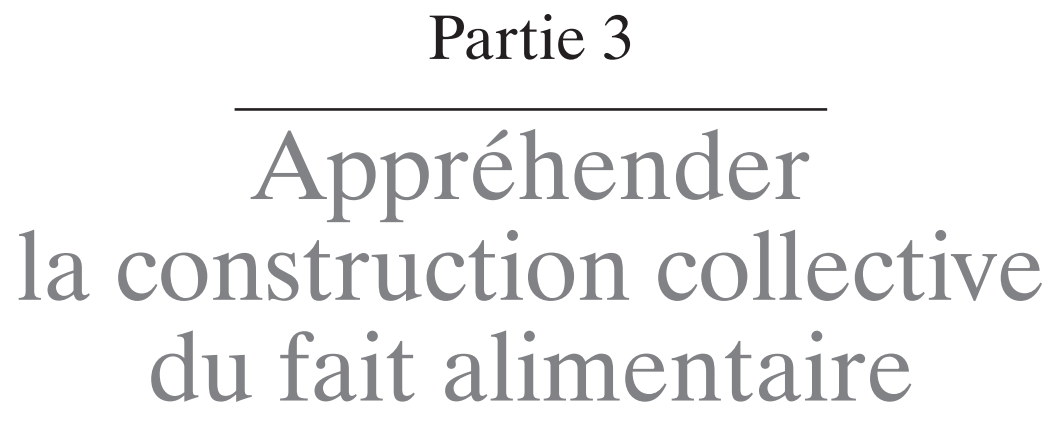





\title{
Urbal : une évaluation participative et qualitative de la durabilité des innovations alimentaires
}

\author{
OLIVIER LEPILLER ${ }^{a}$ É LODIE VALETTE ${ }^{b}$ ALISON BLAY-PALMER, \\ DENIS SAUTIER ${ }^{d}$, MICHAËL BRUCKERT ${ }^{e}$, MARLÈnE PERIGNON $f$, \\ NGUYEN Thi TAN LOC ${ }^{g}$, NGUYEN THI SAUg, \\ OPHÉLIE ROUDELLE ${ }^{h}$ et AMÉLIE WOOD ${ }^{a, i}$
}

\begin{abstract}
a CIRAD, UMR MoISA, F-34398, Montpellier, France; MoISA, université Montpellier, CIHEAM-IAMM, CIRAD, INRAE, Institut Agro, IRD, Montpellier, France.

b CIRAD, UMR Art-Dev, F-34398, Montpellier, France; Art-Dev, université Montpellier, CIRAD, CNRS, université Perpignan, université Paul Valéry, Montpellier, France.

${ }^{c}$ Wilfrid Laurier University, Laurier Centre for Sustainable Food Systems, Waterloo, Canada.

${ }^{\mathrm{d}}$ Innovation, Université Montpellier; CIRAD, INRAE, Institut Agro, Montpellier, France.

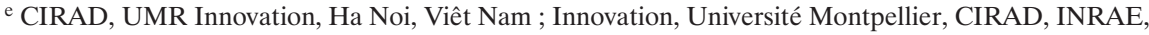
Institut Agro, Montpellier, France.

${ }^{\mathrm{f}}$ MoISA, université Montpellier, CIHEAM-IAMM, CIRAD, INRAE, Institut Agro, IRD, Montpellier, France. g Fruit and Vegetable Research Institute, Ha Noi, Viêt Nam.

h Chaire Unesco Alimentations du monde, université Montpellier, CIRAD, Institut Agro, Montpellier, France.

i LISIS, université Gustave Eiffel, CIRAD, Champs-sur-Marne, France.
\end{abstract}

La méthode Urbal s'intéresse aux impacts des activités que les innovations sociales œuvrant pour une alimentation plus durable mettent en place. Dans le but d'accompagner la transition vers des systèmes alimentaires plus durables, elle propose un suivi-évaluation qualitatif, basée sur la notion de chemins d'impacts. Elle s'appuie sur une démarche participative à laquelle la diversité des acteurs concernés, y compris les mangeurs, est associée pour apporter leur connaissance et leur expérience des impacts.

Les systèmes et régimes alimentaires qui dominent dans les zones les plus riches de la planète ont de nombreuses conséquences négatives, tant sur le plan environnemental que sanitaire, économique, social ou politique. Ils soulèvent des questions de durabilité, dans un contexte de croissance démographique mondiale (Esnouf et al., 2011). Les systèmes alimentaires urbains, qui concentrent les besoins, mais peu les moyens de production, soulèvent des questions particulièrement critiques. Des grandes villes de tous les continents ont signé en 2015 le Milan Urban Food Policy Pact (MUFPP) pour affronter ces questions, partager les solutions expérimentées par les unes et les autres, et se fixer des objectifs communs en termes de durabilité. En effet, l'idée selon laquelle bon nombre des solutions pour des systèmes alimentaires plus 
durables se situeraient à un échelon local ou régional est aujourd'hui fortement défendue. Les villes fourmillent ainsi d'initiatives et d'expériences, dont la contribution à la transformation des systèmes alimentaires pose question. Comment évaluer les impacts de ces innovations et comment accompagner leur changement d'échelle et la transition vers une plus grande durabilité (Geels et Schot, 2007)?

C'est la question à laquelle est dédiée Urbal ${ }^{56}$, qui propose une méthode d'évaluation des impacts des innovations sociales urbaines sur la durabilité des systèmes alimentaires. Cette méthode, développée et testée depuis 2018 dans une quinzaine de cas d'étude sur quatre continents ${ }^{57}$ (Valette et al., 2020), s'adresse aux acteurs de la transition (acteurs des innovations, bailleurs, décideurs politiques).

L'objectif de la démarche n'est pas de se focaliser sur l'atteinte ou non d'objectifs prédéfinis, ni de quantifier ses effets à moyen et long terme : au lieu de chercher à caractériser ce qui a ou n'a pas fonctionné, l'on se concentre plutôt sur les raisons de la réussite ou de l'échec, et sur les processus du changement. Les relations causales précises entre ce que font les innovations et les changements qu'elles produisent restent en effet souvent implicites. Le but est de les expliciter en dépliant les chaînes causales qui conduisent des activités aux changements de long terme. Pour cela, il est nécessaire de distinguer les effets directs (produits ou outputs), de moyen terme (résultats ou outcomes) et de plus long terme (impacts), tout en identifiant les conditions du passage d'une étape à l'autre. Les effets positifs et négatifs, visés ou imprévus sont considérés. Des conditions de réussite nécessaires ou facilitatrices, des obstacles ou des freins peuvent alors être identifiés. Urbal appartient de ce fait à la famille des approches de la «théorie du changement» (Mayne, 2011). La méthode ImpresS développée au Cirad pour documenter les impacts des programmes de recherche et de développement auxquels l'institut participe est une source d'inspiration (Blundo Canto et al., 2020).

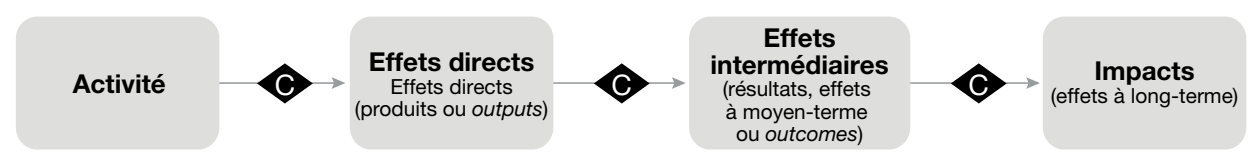

C = conditions de réussite nécessaires ou facilitatrices pour le passage
à l'étape suivante / leviers, freins, points de vigilance, de blocage...

Figure 12.1. Représentation d'un chemin d'impact.

La dimension participative ${ }^{58}$ s'appuie sur l'intelligence collective. Concrètement, cela prend la forme d'ateliers multi-acteurs. L'idée générale est que la diversité des

56. Le projet Urbal ( ${ }^{\circ}$ FC 2015/2440; n FDNC Ellgt 00063479) est financé par le programme Thought for Food Initiative d'Agropolis Fondation (via le programme Investissements d'avenir, ANR10-LABX-0001-01), la Fondazione Cariplo et la Daniel and Nina Carasso Foundation.

57. Voir le site www.urbalfood.org (dernière consultation le 30/08/2021).

58. Cousins et Earl (1992) ont proposé de définir l'évaluation participative comme une «recherche en sciences sociales appliquées, qui implique un partenariat entre des professionnels formés à l'évaluation et des décideurs ayant un rapport pratique à l'objet évalué, des membres de son organisation possédant des responsabilités, ainsi que d'autres personnes ayant un intérêt vital dans le programme de l'objet évalué » (p. 399, notre traduction). Parmi ces dernières, nous incluons les mangeurs, bénéficiaires finaux des innovations étudiées dans Urbal. 
regards et des expériences des acteurs concernés par l'innovation, ainsi que leur confrontation, permettent d'appréhender une gamme d'impacts étendue. Le défi est de réussir à faciliter l'expression des points de vue des acteurs qui ont le plus de difficultés à prendre la parole.

La durabilité des systèmes alimentaires est considérée sous un large spectre de dimensions : environnementale, économique, socioculturelle, politique et sanitaire. Considérer les impacts des innovations sous cet angle multidimensionnel peut conduire à mettre au jour des synergies, mais aussi des contradictions entre dimensions. Par exemple, l'approvisionnement auprès de producteurs locaux, qui est bénéfique à l'économie du territoire et favorise l'insertion socioprofessionnelle de ces derniers, peut s'avérer négatif sur le plan environnemental s'il implique une logistique mal optimisée. Ce type d'observations permet d'adapter, en connaissance de cause, la prise de décision quant aux compromis à adopter et à la hiérarchisation des priorités.

La méthode se veut économe et suffisamment souple pour pouvoir s'adapter à différents cas d'innovations. Celles-ci sont, par définition, des organisations nouvelles, dont l'existence peut être fragile, vacillante. Leurs porteurs dédient beaucoup de leur énergie à pérenniser leur existence. Ils disposent de peu de temps, de moyens financiers, voire de compétences à consacrer à une réflexion sur leurs impacts, étant plutôt centrés sur le cheminement vers leurs objectifs. Le suivi-évaluation des impacts, tel qu'il est couramment pratiqué, est le plus souvent quantitatif, ce qui implique de choisir, voire de construire des indicateurs, une métrique, et de collecter des séries de données. Par exemple, les 17 objectifs de développement durable adoptés par l'Organisation des Nations unies en 2015 ont été traduits en plus de 230 indicateurs. Ce genre d'approche est impraticable pour la plupart des innovations, sauf peut-être par celles qui sont portées par des institutions puissantes. Urbal offre une alternative, sans pour autant l'exclure, à l'approche quantifiée. Le suivi-évaluation qualitatif en termes de chemins d'impacts peut en effet s'articuler à une évaluation quantitative en aidant à identifier les indicateurs à prioriser et en appuyant cette priorisation sur la pertinence sociale.

Le guide méthodologique Urbal sera mis en ligne à disposition de toutes et tous sous licence Creative Commons. La méthode sera ainsi librement appropriable par des porteurs d'innovations, par des décideurs politiques, par des bailleurs cherchant à soutenir des innovations, ou par des chercheurs. Des conditions minimales de bonne utilisation seront précisées (par exemple, l'invitation d'une diversité minimale de parties prenantes lors des ateliers ou la certification que des impacts négatifs n'ont pas été invisibilisés dans les comptes rendus). L'utilisation d'Urbal peut avoir plusieurs objectifs : informer la conduite d'une innovation et la prise de décision quant à son soutien, la promouvoir ou encore préparer une évaluation quantifiée des impacts. Le caractère ouvert ne relève pas que d'un choix politique : il est aussi pragmatique, au service du changement d'échelle des innovations.

Dans ce chapitre, nous présenterons dans un premier temps la méthode, puis nous aborderons ses atouts et limites, ses implications juridiques et éthiques et son articulation avec une approche holistique de l'alimentation. Dans la dernière section, nous exposerons deux cas d'application pour montrer comment la méthode a été adaptée aux contraintes du terrain pour favoriser la participation des mangeurs au suivi-évaluation. 


\section{" Les trois étapes de la méthode Urbal}

La méthode s'organise en trois étapes successives.

La première est dédiée à la caractérisation de l'innovation à partir de ses activités innovantes. Elle se base sur l'analyse de la documentation disponible, une revue de la littérature relative au type d'innovation étudiée et des entretiens avec des personnesressources (porteurs de l'innovation, bénéficiaires ou usagers par exemple). Cette première étape permet de produire un chronogramme de l'innovation retraçant son existence, une carte de ses acteurs et une carte de ses activités.

La seconde étape consiste à organiser un atelier participatif réunissant des acteurs-clés de l'innovation (porteurs, membres, acteurs impliqués dans les activités innovantes, bénéficiaires, usagers, soutiens, acteurs du système alimentaire connecté à l'innovation comme des fournisseurs ou producteurs, voire acteurs d'innovations similaires ou alliées, etc.). L'atelier a pour objectif une cartographie cognitive des chemins d'impacts d'une sélection d'activités caractéristiques de l'innovation. Les participants, orientés par un facilitateur, répondent à la question très simple : que fait l'innovation, concrètement, et qu'est-ce que cela change, a changé, ou peut changer? Le périmètre des participants est déterminé au cas par cas et selon les contraintes de participation des uns et des autres, avec l'objectif général de réunir la plus grande diversité de points de vue sur l'innovation et d'expériences de ses activités. Des experts, spécialistes de plusieurs dimensions de la durabilité des systèmes alimentaires, sont également invités à participer. Il est demandé à ces derniers de se comporter moins en dépositaires d'un savoir surplombant qu'en participants vigilants, aidant à l'explicitation détaillée des chemins d'impacts, à la prise de parole de tous et aux traitements des impacts les moins spontanément abordés. Les personnes en charge de l'organisation doivent être formées à l'animation d'ateliers participatifs et veiller à la logistique de l'atelier (invitations, location et aménagement de la salle, achat et mise à disposition du matériel, sans oublier l'organisation d'un moment de convivialité $)^{59}$.

La phase d'analyse du matériau collecté lors de l'atelier est cruciale. En sortie d'atelier, ce matériau est brut. Généralement, il prend la forme de grandes feuilles A0 sur lesquelles les résultats des discussions ont été représentés. Des enregistrements sonores et des notes peuvent le compléter. Mais, à ce stade, on est encore loin de représentations graphiques propres, détaillées et facilement lisibles des chemins d'impacts. Lors de la phase d'analyse et de mise en forme graphique, les personnes en charge peuvent décider de se cantonner à ce qui a été dit durant l'atelier en le détaillant (par exemple, en explicitant des étapes causales ou des conditions de réussite non formulées) ou décider d'enrichir les chemins d'impacts identifiés en se basant sur la littérature scientifique. Il est aussi possible de garder la trace de qui a dit quoi et a identifié quels impacts. De ce point de vue, il n’y a pas de fétichisation du caractère participatif de la méthode.

\footnotetext{
59. Les méthodes participatives sont très diverses, tout comme leurs contextes d'application et les questions qu'elles traitent. Les publications qui les présentent et les formations qui les enseignent le sont tout autant. Les publications synthétiques manquent. La fondation Roi Baudouin a cependant édité un document de synthèse en accès libre, orienté vers la pratique, qui nous paraît une bonne porte d'entrée dans l'approche participative (Slocum et al., 2006). Un état des lieux de la recherche participative en France a été publié en 2013 par la fondation Sciences citoyennes (Storup et al., 2013). Voir également le chapitre 13 dans ce livre.
} 
La troisième étape vise à restituer les résultats produits à l'issue de l'étape précédente et à les discuter. Cette étape peut prendre des formes variées. Il peut s'agir d'un atelier participatif réunissant les mêmes participants que lors de l'atelier de l'étape précédente, avec éventuellement des participants supplémentaires. Cela peut aussi prendre la forme d'une réunion plus courte et moins participative, mais ménageant tout de même un temps de discussion, notamment pour laisser de l'espace à l'expression des points de vue sur la pertinence des résultats produits et la formulation des chemins d'impacts. La forme à donner à cette troisième étape dépend finalement de la fonction que le commanditaire souhaite privilégier : s'agit-il avant tout d'informer la prise de décision collective relative au fonctionnement et à la gouvernance de l'innovation? De la faire connaître et de la promouvoir, voire de susciter des soutiens politiques ou financiers? De construire ou de solidifier un réseau d'initiatives au sein d'un territoire? De préparer une évaluation quantifiée des impacts et d'identifier leurs indicateurs?

\section{Atouts et limites d'Urbal}

\section{Atouts}

La méthode est conçue pour répondre à une question relativement simple et pratique : quels sont les impacts des innovations alimentaires visant la durabilité ? Cette question découle d'un besoin de suivi et d'anticipation sur les conséquences des activités. Urbal permet de produire un suivi-évaluation in itinere de cette action, capable de saisir des changements ex post, lorsqu'ils sont déjà advenus et observables, ou ex ante, lorsqu'ils sont poursuivis, envisageables ou potentiels. Il est important de distinguer les changements advenus de ceux qui pourraient advenir, dans la mesure où les uns et les autres n'impliquent pas les mêmes ajustements en termes d'action et de décision : approfondissement ou changement d'orientation d'un côté, anticipation pour favoriser ou éviter de l'autre.

Les connaissances produites par Urbal ont vocation à être partagées. Ce partage favorise l'échange d'expériences positives ou négatives, qui est utile, par exemple, à des innovations plus récentes s'inspirant d'autres plus anciennes et plus installées. La philosophie générale, traduite dans le choix d'une propriété intellectuelle Creative Commons, est celle qui préside aux communs intellectuels : «ces ressources immatérielles liées aux savoirs et aux procédures, partagent des caractéristiques similaires avec les ressources communes naturelles telles que les problèmes de passager clandestin, les difficultés d'exclusion, ou encore le risque de dégradation de la ressource selon l'usage qui en est fait. Mais ces ressources ont également des propriétés uniques. Sous certains aspects, elles sont non rivales, dans la mesure où leur construction repose sur des savoirs dont l'usage par un bénéficiaire ne prive pas d'autres utilisateurs [...]. Le gouvernement de ces ressources a comme objectif davantage leur amélioration et leur extension que leur préservation [...]. Leur dégradation est liée à leur non-adaptation aux usages, ce qui pose la question de leur renouvellement» (Lemeilleur et Allaire, 2018, p. 9).

L'objectif est de participer à la construction d'une culture commune autour des solutions pratiques pour des systèmes alimentaires plus durables. Il est aussi de 
produire des connaissances utilisables dans le cadre des différentes formes de changement d'échelle (Lepiller et Valette, 2021). À travers le partage d'expérience, Urbal peut être utilisé au service de la réplication ou l'essaimage (scaling out). Les connaissances qui alimentent la conduite des innovations ou leur accompagnement politique et financier favorisent leur institutionnalisation (scaling up). Via leur diffusion, ces connaissances permettent aussi d'imprégner la culture commune et de faire valoir des idées nouvelles (scaling deep). L'utilisation d'Urbal dans plusieurs cas d'innovations au sein d'un territoire peut aussi favoriser la mise en réseau et le maillage territorial des initiatives.

La dimension participative de la méthode permet de prendre une distance critique vis-à-vis des objectifs des porteurs d'innovations et de s'extraire de la pensée votive (wishful thinking) qui peut les caractériser en favorisant l'expression d'autres impacts que ceux attendus et poursuivis : les impacts surprenants, négatifs ou contradictoires avec d'autres ont ainsi plus de chance d'être mis en lumière. La participation assure une certaine pertinence sociale au suivi-évaluation, puisque les impacts exprimés sont ceux qui comptent pour les participants. À ce titre, la participation des experts est importante. Ces derniers sont invités à jouer un rôle auxiliaire dans la formulation des impacts et chemins d'impacts, ainsi que dans l'apport d'informations utiles à la discussion et dans la formulation éventuelle d'impacts qui ne ressortiraient pas spontanément des discussions.

\section{Limites}

Les limites classiques des méthodes participatives sont aussi celles de la méthode. Il peut s'avérer difficile de faire participer des parties prenantes, pourtant concernées par l'innovation, qui ne se jugent pas légitimes à prendre la parole. Parfois, ce sont les compétences mêmes de certains acteurs qui empêchent la participation, ou obligent à en repenser les formes. Comment, par exemple, faire participer de jeunes enfants, ou des personnes non alphabétisées, à des ateliers?

La libre expression des participants à un atelier peut aussi être entravée par leurs attachements institutionnels, comme lorsqu'ils sont tenus par un devoir de réserve, ou que leur hiérarchie leur interdit de participer. Dans ce type de situation, la préparation, la prise de contact et l'explication des objectifs en amont de l'atelier apparaissent cruciales pour rassurer les acteurs peu disposés à participer. Le dispositif participatif doit aussi être pensé pour contenir l'enthousiasme de certains acteurs, comme les porteurs d'innovation qui, animés par l'engagement dans leur action, peuvent occuper beaucoup de place lors des ateliers. Il doit aussi tenir compte des asymétries sociales (dominant/dominé, supérieur/subordonné, etc.) Les rôles des experts invités et des facilitateurs des ateliers doivent être pensés en lien avec cette problématique.

Une autre limite se situe dans la réception de ses résultats, plus précisément de leur légitimité. Ces résultats, qualitatifs, ne sont pas mis en mesures métriques. Les indicateurs chiffrés apparaissent souvent comme les plus légitimes, car jugés plus «objectifs». Or, la valeur des résultats d'Urbal se mesure plus à l'aune de leur pertinence sociale et de leur pouvoir explicatif de la causalité des impacts qu'à celle d'une objectivité métrée. Pour faire face à ce défi de légitimité, l'équipe du projet 
travaille au développement d'outils de communication graphique simples et efficaces, favorisant une intelligence rapide de la variété des chemins d'impacts dans les différentes dimensions de la durabilité. Un autre argument en faveur de la légitimité des résultats qualitatifs d'Urbal réside dans la possibilité d'articuler ceux-ci avec une évaluation quantitative, l'application de la méthode constituant dans ce cas une étape préparatoire, socialement pertinente, à la quantification des impacts.

Un dernier ensemble de limites est lié à la volonté de faire d'Urbal une méthode économe en ressources, en temps et en argent. Bien que différentes tailles de voilure soient prévues selon les utilisations possibles, l'objectif est que l'ensemble du processus soit réalisable dans le format minimal d'une mission de trois mois, par exemple via un étudiant en stage de master, ou un chargé de mission. Cette exigence d'économie implique des arbitrages. Le temps de l'atelier participatif peut s'avérer difficile à organiser en fonction des contraintes d'agenda, de mobilité, d'intérêt ou de liberté de parole des participants. Il doit donc être choyé pour en tirer le plus d'informations possible quant aux chemins d'impacts. Il est possible d'identifier au préalable les activités innovantes dont les impacts seront discutés. Selon les innovations, les activités peuvent être nombreuses, mais en aborder plus d'une dizaine au cours d'un atelier de quatre heures apparaît difficile. Il convient alors de choisir les activités les plus emblématiques de l'innovation, ou les plus intéressantes pour telle ou telle dimension de la durabilité, en fonction de telle ou telle question.

Le choix de focaliser ou non sur certaines des dimensions en particulier constitue un second arbitrage, en tension avec l'ambition multidimensionnelle de la méthode. La nature même des innovations peut justifier d'explorer plus en profondeur certaines dimensions plutôt que d'autres. Par exemple, une épicerie solidaire fonctionnant comme un dispositif d'insertion de personnes en situation de précarité peut justifier qu'un temps plus long soit accordé à la discussion collective sur les dimensions économiques, socioculturelles et politiques.

Enfin, un troisième arbitrage porte sur le niveau de détail relatif aux chemins d'impacts attendu en fin d'atelier. Si l'on cherche à couvrir le plus large ensemble possible d'impacts des activités, il s'avère difficile de rentrer dans le détail des chemins d'impacts. Dans ce cas, le matériau collecté en sortie d'atelier s'intéresse plus aux impacts qu'à leurs chemins. Ces derniers peuvent ensuite être formulés et cartographiés dans le détail lors de la phase d'analyse post-atelier, pour être ensuite soumis à la discussion et à la validation des participants dans le cadre de l'atelier de la troisième étape. Mais le choix peut aussi être fait de préparer certains chemins d'impacts en amont de l'atelier participatif de la seconde étape pour les discuter et les valider dans ce cadre, et de n'aborder que plus superficiellement les autres impacts jugés moins cruciaux pour l'innovation ou la question principale qui guide l'évaluation.

\section{Implications juridiques et éthiques d'Urbal}

Comme toute enquête scientifique impliquant le recueil de discours, la méthode est soumise au respect des lois en vigueur dans le lieu où elle est mise en pratique. En Europe, il s'agit du règlement général sur la protection des données (RGPD), qui nécessite de faire une demande préalable d'autorisation du protocole 
d'enquête et du plan de traitement des données. Selon le niveau de précision voulu, il n'est pas forcément nécessaire de garder la trace de qui a dit quoi lors des ateliers, donc l'attribution de discours à des acteurs précis, anonymisés ou non, n'est pas une obligation. L'anonymisation, d'ailleurs, peut s'avérer illusoire dans certains cas. La mention d'un poste, par exemple celui de directeur de la restauration scolaire d'une ville, peut suffire à identifier une personne sans même préciser son nom. Il est important d'en informer au préalable les participants aux ateliers et d'obtenir leur accord.

Au niveau éthique, la mise en œuvre d'Urbal se doit de favoriser la prise de parole des acteurs qui y accèdent le plus difficilement. Lorsque ces acteurs, qu'ils soient en situation de domination, de subordination, d'empêchement par la hiérarchie, de manque de compétences ou d'auto-exclusion, ne peuvent participer à l'atelier pour une raison ou une autre, il est alors possible d'envisager de recueillir leur parole par d'autres moyens, pour se prémunir des effets de captation illégitime de la parole par d'autres, lors de l'atelier.

\section{W Urbal et l'approche holistique de la durabilité des systèmes alimentaires}

L'approche holistique de la durabilité des systèmes alimentaires est au fondement de la méthode, qui cherche à identifier des chemins d'impacts dans ses différentes dimensions. Malgré tout, et comme nous l'avons évoqué, des choix peuvent être faits, par souci d'efficacité, pour focaliser l'enquête plus spécifiquement sur telle ou telle dimension, tel ou tel ensemble d'impacts. En pratique, l'approche holistique se matérialise dans l'organisation des ateliers, via l'invitation d'une diversité d'acteurs aux intérêts et aux points de vue différents sur l'innovation, et via l'invitation d'experts en différentes dimensions de la durabilité des systèmes alimentaires. Si l'ensemble des dimensions de la durabilité n'est pas nécessairement représenté par des experts, il importe que plusieurs d'entre elles le soient, en invitant par exemple un expert en nutrition, un autre en économie, un troisième autour des enjeux sociaux. Cela implique de faire participer des experts de différentes disciplines.

Les résultats obtenus pour une innovation donnée conduisent à étiqueter les différents impacts des activités selon les dimensions de la durabilité. Cela permet de visualiser les dimensions qui entretiennent des affinités. Un impact, par exemple, peut se situer à la fois dans l'ordre économique et socioculturel, comme lorsque la contractualisation avec un producteur de proximité lui permet de gagner en compétences professionnelles, d'augmenter ses revenus sur le long terme et de s'insérer socialement dans un collectif. L'attribution des impacts aux différentes dimensions permet aussi de visualiser les éventuelles contradictions entre elles. Par exemple, une mesure favorable à l'environnement peut s'avérer néfaste en matière de santé nutritionnelle, comme lorsque des produits végétaux alternatifs à des aliments carnés s'avèrent de plus faible qualité nutritionnelle. La visualisation de ces synergies et contradictions alimente la prise de décision et rétablit la dimension politique des innovations et de leur quête de durabilité, au sens 
où les choix, donc la hiérarchisation des priorités, les arbitrages et les abandons, éventuellement temporaires, sont rendus manifestes. En cela, Urbal est au service d'une vision pragmatique, plutôt qu'idéalisée, de la durabilité : une vision qui privilégie les itinéraires de changement choisis en connaissance de cause grâce à l'intelligence collective.

\section{Adaptations de la méthode pour impliquer les mangeurs dans l'évaluation}

Les innovations sociales étudiées pour développer Urbal ont été choisies pour offrir une variété de situations. Citons, entre autres :

- des innovations permises par les nouvelles technologies (par exemple la commande en ligne de produits alimentaires de qualités spécifiques répondant à des enjeux de durabilité à Hanoï);

- des innovations portées par des collectifs engagés (par exemple une épicerie solidaire à Paris; un supermarché coopératif à Montpellier; un système participatif de garantie de la qualité agroécologique à Rabat);

- des innovations portées par des entrepreneurs de l'alimentation durable (par exemple une production de tortillas de maïs de variétés indigènes cultivées par des producteurs locaux et transformées selon un procédé traditionnel à Mexico City; un site de production aquaponique de produits végétaux et de poissons vendus localement à Berlin);

- des innovations portées par des institutions (par exemple un programme d'amélioration de la restauration scolaire à Montpellier; un conseil citoyen de l'alimentation à Baltimore; un district alimentaire qui vise à promouvoir l'approvisionnement et l'agriculture locaux à Milan; une stratégie de lutte contre l'insécurité alimentaire au Cap).

Nous nous centrerons sur deux cas d'application pour illustrer la manière dont les mangeurs sont associés à la démarche de suivi-évaluation et montrer leurs apports au processus.

Le premier s'est déroulé à Hanoï, capitale du Vietnam. Il s'intéressait non pas à une innovation aux contours bien définis d'un point de vue institutionnel, mais plutôt à une innovation d'usage liée aux nouvelles technologies de communication : l'utilisation des réseaux sociaux (Facebook ou Zalo, un réseau vietnamien très utilisé, notamment) pour commander des produits alimentaires de qualités spécifiques répondant à des enjeux de durabilité. Dans un contexte vietnamien marqué par un développement rapide des supermarchés et des filières alimentaires industrialisées depuis le début des années 2000, le commerce de ces aliments de qualité plus durable est relativement récent. Le développement de leur commerce en ligne - distinct du e-commerce de masse de la grande distribution -, l'est encore plus. Le concept même de durabilité est d'ailleurs difficilement traduisible et d'un usage peu commun en vietnamien. En conséquence, l'équipe de recherche a dû faire le choix de la désignation des produits qui pouvaient répondre à ce qualificatif de durable. Il s'agissait de produits alimentaires bruts (fruits, légumes, viandes, fruits de mer, miel) ou peu transformés (pâtisseries, épices, salaisons) présentant des qualités 
mieux-disantes par rapport à leurs équivalents issus des filières industrialisées : avec des méthodes de cultures ou d'élevage limitant l'utilisation des intrants chimiques de synthèse (labellisés ou non), fabriqués selon des procédés traditionnels limitant l'utilisation d'additifs, marqués par une indication d'origine et une identité culturelle spécifique, et souvent vendus directement par le producteur ou par un intermédiaire unique. Des qualités organoleptiques supérieures sont généralement associées à ces différentes qualités.

Un enjeu important de l'atelier participatif organisé en décembre 2019 était de permettre à des mangeurs d'apporter leur point de vue quant aux impacts des activités suivantes : commander en ligne, payer en ligne, publier des commentaires, poser des questions au vendeur. Dans le contexte du Vietnam, un pays marqué par une culture politique particulièrement dirigiste, avec un exercice du pouvoir très vertical et un grand respect pour l'ordre hiérarchique, cette participation n'allait pas de soi. Les mangeurs invités devaient pouvoir se sentir légitimes à discuter avec des experts (en agronomie et environnement, indications géographiques et économie agricole), un fonctionnaire du contrôle de la qualité des aliments et des journalistes. Il fallait tout d'abord, pour cela, qu'ils acceptent l'invitation et se rendent à l'atelier. Les chercheurs vietnamiens impliqués dans le projet se sont chargés du recrutement de plusieurs mangeurs et de la prise de contact en respectant les usages, ce qui est notamment passé par l'envoi d'un formulaire de participation imprimé aux mangeurs invités, qui ont également été prévenus qu'un cadeau leur serait offert à l'issue de l'atelier (en l'espèce, des produits alimentaires, comme des oranges produites par un des vendeurs participants). Ils furent aussi invités à partager un repas au restaurant à l'issue de l'atelier. Durant l'atelier, l'un des chercheurs partenaires utilisa ses talents pour l'animation. Maniant l'humour, il insista sur l'idée que l'expérience de chacun des participants avait une valeur pour le suivi-évaluation, et que les experts n'avaient pas été invités pour faire le partage du vrai du faux, mais pour nourrir les discussions, soulever des questions, apporter des précisions. Les partenaires vietnamiens, qui n'avaient jamais organisé d'atelier participatif selon un principe aussi horizontal, se montrèrent heureusement surpris de la participation active des mangeurs aux discussions. En termes de résultats, la participation des mangeurs permit de souligner la centralité de la question de la confiance autour des transactions marchandes en ligne. À ce sujet, les mangeurs pointèrent plusieurs points :

- l'importance des avis ou des expériences de proches dans la construction de la confiance, avant une première transaction avec un vendeur;

- l'importance d'une communication réactive des vendeurs pour répondre aux demandes d'information;

- l'importance des différents supports de communication qui permettent de se représenter l'origine et la fabrication des produits alimentaires, ainsi que leurs producteurs (vidéos, images...), dans une perspective de transparence et de reconnexion avec le monde agricole.

Le second cas d'application a mis en avant d'une autre manière la question de la participation des mangeurs. L'innovation étudiée était le programme d'amélioration de la restauration scolaire de la ville de Montpellier. Nous avons rapidement fait face à cette question : comment recueillir le point de vue des premiers concernés et principaux bénéficiaires, à savoir des enfants scolarisés en école primaire ? Il aurait 
été imaginable d'adapter le dispositif de l'atelier participatif à des enfants âgés d'une dizaine d'années, par exemple à travers des exercices ludiques. Mais il nous a semblé que cela aurait forcé à beaucoup simplifier le registre de la discussion générale, ce qui aurait conduit à perdre en niveau de détail dans l'identification des chemins d'impacts. Par ailleurs, la participation des enfants soulevait des questions juridiques (autorisation des parents, procédure de mise en conformité éthique, etc.), dont la résolution était peu compatible avec l'agenda du projet. Le choix a donc été fait de déroger partiellement à l'esprit participatif de l'atelier en n'y invitant pas d'enfants, mais en recueillant et en rapportant leurs points de vue durant l'atelier et en l'agrégeant à ceux des participants dans l'analyse post-atelier. Pour cela, une enquête qualitative a été conduite auprès d'écoliers dans plusieurs établissements scolaires et au sein du conseil municipal des enfants de la ville de Montpellier. Des entretiens collectifs et individuels, ainsi que des observations ethnographiques lors de temps du repas ont ainsi été réalisés.

Sans cette enquête, qui a permis d'accéder à l'expérience des enfants, certains impacts n'auraient pas pu être identifiés, ou l'auraient été moins précisément. D'une manière générale, la sensibilité des enfants pour les impacts environnementaux est apparue très prégnante, comme en témoignaient leurs jugements relatifs au tri des déchets via des tables de tri affichant les quantités ou à l'augmentation de la part d'aliments biologiques. Au sujet des tables de tri, un enfant enquêté a pointé un phénomène de socialisation inversée, suggérant un impact possible sur les pratiques familiales : «Maintenant, quand ma famille ne jette pas les trucs dans la bonne poubelle, je leur montre comment recycler, et maintenant ils savent». Au sein même du restaurant, la relation de coopération avec les agents de service permise par les tables a été pointée par une enfant comme une source de satisfaction, permettant de se sentir bien en apportant activement de l'aide et en étant félicité. Un autre enfant a néanmoins identifié un impact potentiellement négatif des tables de tri, en confiant le choc ressenti en se rendant compte du volume des déchets, ce qui suggère un besoin d'accompagnement des interprétations de l'activité.

Une autre activité du programme est apparue ambivalente : la mise en place de menus «alternatifs éco-citoyens » sur un rythme mensuel - depuis devenu bi-hebdomadaire. Des enfants ont souligné son caractère plus «écolo» dû à l'absence de viande, la possibilité de «se mettre dans la peau d'un végétarien » et de réaliser qu'on n'avait pas besoin de manger de la viande tous les jours, ou qu'il était possible de faire un repas complet en s'en passant. Un impact en termes d'inclusion sociale a été pointé par un enfant, pour qui ce menu permettait «aux sans-viande de manger comme nous». Les jours de service de ces menus, l'ensemble des enfants mangeaient en effet la même chose, puisque le menu «alternatif éco-citoyen» était compatible avec les trois options habituellement proposées (menus «standards», «sans porc», «sans viande»). Mais des craintes relatives à la satisfaction des apports nutritionnels (fer et protéines) ont aussi été exprimées, ainsi qu'un impact sur la satiété, chez un enfant disant ne plus pouvoir rien manger le jour où le menu «alternatif éco-citoyen » était servi parce qu'il n'aimait pas les légumes.

Enfin, le témoignage des enfants a permis de confirmer et de mieux comprendre les effets, qui avaient déjà été mesurés en termes de baisse des quantités gaspillées, de l'introduction d'un outil permettant la découpe rapide des fruits, notamment des 
pommes, directement à table. Le découpage rapide en quartiers facilitait le partage et permettait aux enfants qui appréhendaient de manger un fruit entier de ne s'en servir que quelques morceaux. L'aspect ludique de cet outil de découpe est apparu encourageant pour la consommation des fruits : «C'est bien car ça fait une fleur quand on coupe, c'est joli et rigolo ».

Pour conclure ce chapitre, pointons l'importance d'associer le plus possible les mangeurs au suivi-évaluation des innovations œuvrant pour une alimentation plus durable. Les intégrer dans le processus participatif enrichit la qualité de l'évaluation, puisqu'à travers l'expérience qu'ils en ont, ils possèdent une connaissance pratique des impacts des activités innovantes. Sur un plan plus politique, faire participer les mangeurs à l'évaluation s'inscrit dans une politisation de l'alimentation et contribue au projet d'une démocratie alimentaire (Paturel et Ndiaye, 2019), tout en favorisant l'émergence de connaissances communes et partagées sur les enjeux des pratiques alimentaires.

\section{" Références}

Barret D., Blundo Canto G., Dabat M.-H., Devaux-Spatarakis A., Faure G., Hainzelin E., Mathe S., Temple L., Toillier A., Triomphe B., 2017. Guide méthodologique ImpresS. Évaluation ex post des impacts de la recherche agronomique dans les pays du Sud, Montpellier, Cirad, 98 p.

https://doi.org/10.19182/agritrop/00005.

Blundo Canto G., de Romemont A., Hainzelin E., Faure G., Monier C., Triomphe B., Barret D., Vall E., 2020. ImpresS ex ante. Démarche pour co-construire ex ante les chemins d'impact de la recherche pour le développement. Guide méthodologique ImpresS ex ante (Deuxième version). Montpellier, Cirad, 76 p. https://doi.org/10.19182/agritrop/00142.

Cousins J. B., Earl L. M., 1992. The Case for Participatory Evaluation. Educational Evaluation and Policy Analysis, 14 (4), 397-418. https://doi.org/10.3102/01623737014004397

Esnouf C., Russel M., Bricas N. (éd.), 2011. Pour une alimentation durable : réflexion stratégique duALIne. Versaille, Quae, (coll. Matière à débattre et décider), 288 p.

Geels F. W., Schot J., 2007. Typology of sociotechnical transition pathways. Research Policy, 36 (3), 399-417. https://doi.org/10.1016/j.respol.2007.01.003

Lemeilleur S., Allaire G., 2018. Système participatif de garantie dans les labels du mouvement de l'agriculture biologique. Une réappropriation des communs intellectuels. Économie rurale. Agricultures, alimentations, territoires, 365, 7-27. https://doi.org/10.4000/economierurale.5813

Lepiller O., Valette E., 2021. Les innovations alimentaires urbaines face aux enjeux de changement d'échelle et d'inclusion sociale. So what? Policy Brief, 14, 1-4. [En ligne] https://www.chaireunescoadm.com/IMG/pdf/01-sowhat-14_2021-fr_10mars.pdf (dernière consultation le 30/08/2021).

Mayne J., 2011. Contribution Analysis: Addressing Cause and Effect, in Forss K., Marra M., Schwartz R. (éd.), Evaluating the Complex. Attribution, Contribution and Beyond, New Brunswick, Londres, Transaction Publishers, 53-96.

Paturel D., Ndiaye P., 2019. Démocratie alimentaire : de quoi parle-t-on? Montpellier, Chaire Unesco Alimentations du monde. [En ligne] https://www.chaireunesco-adm.com/Democratie-alimentairede-quoi-parle-t-on (dernière consultation le 30/08/2021).

Slocum N., Elliott J., Heesterbeek S., Lukensmeye C. J., 2006. Méthodes participatives : un guide pour l'utilisateur, Bruxelles, Fondation Roi Baudouin, 203 p. [En ligne] https://sites.uclouvain.be/ reso/opac_css/doc_num.php? explnum_id=14673 (dernière consultation le 30/08/2021). 
Storup B. (coord.), Millot G., Neubauer C., 2013. La recherche participative comme mode de production de savoirs. Un état des lieux des pratiques en France. Paris, Fondation Sciences citoyennes, 94 p. [En ligne] https://www.open-sciences-participatives.org/uploads/img/resources/5b194c8f780b3 FSC-recherche_participative-FdF-web.pdf(dernière consultation le 30/08/2021).

Valette E., Schreiber K., Conaré D., Bonomelli V., Blay-Palmer A., Bricas N., Sautier D., Lepiller O., 2020. An emerging user-led participatory methodology: Mapping impact pathways of urban food system sustainability innovations, in Blay-Palmer A., Conaré D., Meter K., Di Battista A., Johnston C. (éd.), Sustainable food system assessment: Lessons from global practice, Abington, Routledge, 19-41. https://doi.org/10.4324/9780429439896 



\title{
Chapitre 13 \\ La recherche-action : un processus d'analyse et de transformation sociale pour l'accès à l'alimentation durable
}

\author{
PAULINE SCHERER ${ }^{a}$
}

a LERIS (Laboratoire d'étude et de recherche sur l'intervention sociale), Montpellier, France.

La recherche-action est basée sur la participation et la coopération des habitants, des acteurs «de terrain» et des chercheurs pour produire collectivement des connaissances et des pratiques sociales. Appliquée aux enjeux d'accès à une alimentation durable en situation de précarité, la recherche-action s'avère un outil pertinent pour contribuer à l'émancipation des personnes invisibilisées.

Historiquement, la recherche-action émerge après la seconde guerre mondiale, avec l'idée que les sciences humaines et sociales peuvent jouer un rôle pour répondre aux questions sociétales. Son émergence se situe notamment aux États-Unis et en France. Aux États-Unis, avec la philosophie pragmatiste (William James et James Dewey) qui s'appuie sur le principe d'expérimentation (la valeur d'une théorie est fonction de son efficacité pratique) et avec la psychosociologie (Kurt Lewin), via des dispositifs expérimentaux d'observations et d'analyse comparative des comportements collectifs (notamment en termes d'habitudes alimentaires). En France, c'est au sortir de la guerre qu'elle émerge avec l'analyse institutionnelle, notamment avec Henri Desroche, sociologue, pédagogue et spécialiste des coopératives, qui fonde le bureau d'études coopératives et communautaires en 1953. Alexia Morvan (2013), dans son article de définition de la recherche-action, retrace cette histoire et montre la coexistence de deux modèles distincts. Pour elle, ce qui fonde l'unité des démarches de recherche-action est «qu'elles considèrent l'expérience, l'action (ou l'activité) comme source de connaissance et assument une posture d'engagement du chercheur dans la transformation de la réalité (ou d'efficacité pratique de la recherche)». Elle invite ensuite à différencier, au sein de la recherche-action, des démarches qui relèvent d'une recherche-action appliquée et d'autres qui relèvent d'une recherche-action participative (ou recherche impliquée). Les premières visent à produire un savoir utile dans l'action, sans nécessairement remettre en cause la différence de statut, au regard de la production des connaissances, entre le chercheur (avec son savoir théorique) et les autres participants (les praticiens avec leurs 
savoirs pratiques). Les secondes contestent, au contraire, la frontière entre théorie et pratique et remettent en cause le pouvoir du chercheur et son statut de seul producteur des savoirs légitimes. La recherche-action porte en elle la capacité d'agir sur les rapports sociaux (de classes, de genres, etc.) et sur les dynamiques de justice sociale et de démocratie. Elle possède ainsi une dimension fondamentalement politique, dont Alexia Morvan situe les origines, dans l' «analyse institutionnelle », un courant de recherche en sciences humaines et sociales, principalement en sociologie, qui s'est construit en France à partir des années 1960. Ce courant s'intéresse aux processus par lesquels les rapports de pouvoir et les règles de la vie collective sont normalisés au point qu'ils vont de soi et ne sont plus questionnés, mais aussi aux processus de changement et de prise de conscience de ces règles et rapports de pouvoir, dans une visée critique et émancipatrice. L'analyse institutionnelle est en effet née d'interventions sur des terrains de critiques sociales : «ces travaux ont en commun de créer des cadres de collaboration entre chercheurs et groupes concernés avec le souci que ces derniers fassent autorité sur le devenir des situations à partir des connaissances produites sur celles-ci [...] ce modèle défend l'implication des acteurs [dans le] processus de production des connaissances, mais aussi sa finalité de transformation sociale. Il s'accompagne de la reconnaissance de la fonction politique de toute recherche en sciences sociales» (Morvan, 2013). La recherche-action connaît aujourd'hui de multiples usages, mais «son utilisation reste suspecte dans les sciences humaines dites nobles du fait de l'implication reconnue du chercheur», élément auquel s'ajoute un questionnement sur la scientificité d'une recherche menée avec des praticiens et en partie par eux. L'implication du chercheur, doublée de la portée transformatrice de la recherche-action, soulève plusieurs enjeux quant aux risques d'instrumentalisation, d'asymétries de légitimité entre savoirs académiques et profanes, de co-production inégalitaire des savoirs et de répartition des «bénéfices» des connaissances produites. Ces enjeux doivent conduire à interroger en permanence, pour les ajuster, les manières de poser, de concevoir, d'animer, et de réaliser une recherche-action.

Dans le champ de la sociologie académique, la recherche-action possède des affinités avec la méthode d'intervention sociologique développée par Alain Touraine (1978) dans son ouvrage La voix et le regard. Cette méthode a introduit une posture du sociologue rompant «avec la posture traditionnelle et académique de la neutralité axiologique qui attend du sociologue qu'il se tienne en retrait et se contente d'enregistrer des opinions et des témoignages» (Cousin et Rui, 2010). Celle-ci renvoie cependant plus à l'étude des mouvements sociaux qu'à un objectif de transformation sociale, qui est l'une des perspectives de la recherche-action.

Selon Yves Bonny, si la recherche-action est une notion «vague, que ce soit quant au sens à donner au trait d'union ou quant à la conception même des termes mis en relation», c'est parce que, historiquement, ses «contextes d'émergence sont on ne peut plus variés : contextes académiques, mais aussi contextes militants, de management social, de formation, de développement personnel ou professionnel, de démarches participatives». Ainsi, la recherche-action "peut être abordée en tant que méthode scientifique par certains, associée ou non à des visées très directement opérationnelles, de type résolution de problème. Elle peut viser à formaliser, dans une perspective de transmission et de diffusion, des orientations d'action et d'organisation jugées pertinentes dans un milieu de pratique donné, tel que l'éducation 
populaire [...]. Elle peut s'inscrire plutôt dans une entreprise de conscientisation à l'égard de certaines formes d'oppression et de mobilisation politique, orientée vers le développement du pouvoir d'agir et la transformation sociale. Elle peut relever de perspectives stratégiques de type technocratique ou, au contraire, de dynamiques participatives et coopératives» (Bonny, 2014, p. 1). Bonny montre par ailleurs que la recherche-action peut être abordée de deux points de vue qui s'articulent : premièrement, une recherche à partir de l'action, c'est-à-dire prenant pour objet d'étude une pratique (professionnelle, militante, etc.) ou certaines caractéristiques d'un milieu de la pratique; deuxièmement, une recherche en vue de l'action, c'est-à-dire se donnant explicitement pour visée de produire des connaissances directement en prise sur des finalités pratiques. Les connaissances produites par la rechercheaction, connaissances «décontextualisables » et connaissances «actionnables », sont cumulables, mais peuvent entrer en tension. Celle-ci peut se révéler stimulante si elle est explicitée, sans quoi elle risque de générer conflits et malentendus dans le cadre de recherches partenariales.

La dimension participative et coopérative abordée par Bonny désigne la mise en relation et l'implication de l'ensemble des parties prenantes liées au sujet abordé, en premier lieu les «premiers concernés ». La notion de coopération entend rassembler différents éléments : la participation des premiers concernés, la mise en commun des ressources, l'implication du chercheur, le croisement des savoirs, la recherche de relations égalitaires et non concurrentielles entre les parties et les savoirs, la recherche de partage des bénéfices.

Le programme de recherche, dont deux cas d'application sont exposés dans ce chapitre, s'inscrit dans une recherche-action dite «coopérative», qui vise la co-production de connaissances, le renforcement des capacités et de la puissance d'agir, la transformation des rapports sociaux et le renforcement de la démocratie (Maurel, 2010). Par ailleurs, cette recherche-action s'attache à un principe d'expérimentation reposant sur l'idée que les sciences humaines ne se tiennent pas à distance des enjeux sociaux et politiques, en référence aux travaux du sociologue Pascal Nicolas-Le Strat. Selon lui, la sociologie «investit expressément [ces enjeux] en s'associant aux expérimentations dans lesquelles s'aventurent les collectifs en vue d'une transformation émancipatrice des formes de vie et d'activité » (NicolasLe Strat, 2013, p. 9). Dans une logique de résistance et de transformation sociale, il s'agit d'opposer au modèle majoritaire de la recherche des pratiques différentes, qui articulent les enjeux politiques avec les expériences de vie et d'activité, et redonnent de la valeur à l'expérience. Expérimenter, selon Nicolas-Le Strat, c'est introduire une perturbation dans une routine. Cette perturbation va réengager les acteurs et produire potentiellement une dynamique constituante, génératrice d'autres rapports sociaux. Ce qu'il nomme la «recherche en situation d'expérimentation [...] revêt une portée à la fois critique, en assumant autant que nécessaire son engagement oppositionnel, et contributive en apportant sa pierre à ces mobilisations collectives en quête et en conquête d'une plus grande autonomie de vie» (Nicolas-Le Strat, 2013, p. 10). La notion d'expérimentation renvoie donc plus à la manière dont les chercheurs et chercheuses sont en capacité de s'associer aux expérimentations sociales en y apportant leur contribution spécifique, qu'à des dispositifs expérimentaux d'observations et d'analyse tels que développés par Kurt Lewin, même si, comme nous le verrons, les deux peuvent parfois se mêler. 
Les exemples qui suivent montrent la manière dont la recherche-action peut être utilisée, en liaison avec des expérimentations, pour traiter le sujet de la précarité alimentaire, travailler le dépassement des dispositifs et pratiques d'aide alimentaire, questionner l'égalité d'accès à l'alimentation durable ${ }^{60}$ et la conciliation entre enjeux écologiques et de justice sociale. Elle est appréhendée en tant que démarche capable à la fois de produire des connaissances et des pratiques sociales, et de contribuer à des dynamiques d'émancipation et de transformation sociale.

\section{\# Recherche-action et accès à l'alimentation durable}

La recherche-action s'intéresse particulièrement à «des mondes invisibles ou peu reconnus» (Morvan, 2013) ou, pour le dire autrement, à des mondes subalternes ou des publics en situation de domination. C'est pourquoi elle est particulièrement développée et pertinente sur des questions qui touchent aux inégalités sociales d'accès à des aliments de qualité durable. En effet, ces aliments (par exemple biologiques ou issus de circuits courts) sont en général plus chers. Ils sont plus consommés par les catégories sociales les plus aisées et les plus instruites. Ainsi, ces aliments, pourtant réputés meilleurs pour l'environnement et pour la santé, sont difficilement accessibles pour les plus pauvres et ils sont quasiment absents de l'aide alimentaire.

Cette approche induit un questionnement sur les situations de précarité alimentaire, sur les pratiques alimentaires corrélées aux conditions de vie, sur les modes d'accès à l'alimentation, sur les modèles et dispositifs de solidarité et de protection sociale, sur la durabilité des systèmes alimentaires, et in fine sur le droit à l'alimentation et la démocratie alimentaire (Paturel et Ndiaye, 2020) qui s'articule aux enjeux de justice sociale (Fraser, 2014) et d'émancipation (Maurel, 2010). L'apport de la recherche-action sur les questions alimentaires concerne, dans les cas présentés dans ce chapitre, sa dimension politique. Elle vise à saisir des pratiques en mouvements, tout en cherchant à les outiller et potentiellement à les renforcer pour favoriser l'égalité d'accès à une alimentation durable. À cette fin, l'observation et la connaissance fine des pratiques alimentaires constituent un socle indispensable au processus de recherche-action.

Différents programmes de recherche-action se sont intéressés ces dernières années à la question de l'accès des personnes en situation de précarité à l'alimentation durable, s'inscrivant en creux dans une approche critique du système d'aide alimentaire sur différents plans : nutrition et qualité de l'alimentation, dignité et citoyenneté des personnes, durabilité des systèmes alimentaires et protection sociale des personnes vulnérables. De nombreuses expériences de recherche ont été conduites aux ÉtatsUnis autour de la justice alimentaire et de l'agriculture urbaine, ou au Québec autour

60. Par «alimentation durable», nous désignons une alimentation qui prend en compte des contraintes dont le respect permet d'assurer un accès pérenne à une alimentation de bonne qualité. Ces contraintes sont d'ordre environnemental, économique, socioculturel, sanitaire et politique. Une alimentation durable est une alimentation qui ne dégrade pas l'environnement, qui assure des revenus justes aux acteurs économiques, qui est adaptée aux identités et aux goûts des mangeurs, qui prend soin de la santé et sur laquelle les acteurs concernés peuvent exercer un pouvoir de décision. Laccès à une alimentation durable est un enjeu majeur des situations de précarité. Il soulève la question de la dignité, de l'égalité et du pouvoir d'agir. 
des systèmes alimentaires territoriaux et de la lutte contre la pauvreté. En Belgique, une recherche-action réalisée en «co-création», intitulée Solenprim ${ }^{61}$, a été conduite par des organisations d'aide alimentaire, une plateforme d'achats solidaire et la Fédération des services sociaux. En France, nous pouvons citer les travaux de l'unité de recherche Innovation, à Montpellier, autour des circuits-courts et de la cohésion sociale, ou de l'approvisionnement en fruits et légumes locaux des Restos du cour de l'Hérault (Paturel, Le Velly, 2014); la recherche-action Accessible, menée par le réseau national des Centres d'initiatives pour valoriser l'agriculture et le milieu rural (Civam) ${ }^{62}$; la recherche-action Formes urbaines et gouvernance alimentaire (Frugal) ${ }^{63}$, qui porte sur l'analyse des enjeux systémiques liés à l'approvisionnement alimentaire de métropoles du Grand Ouest français et de Rhône-Alpes.

Dans la suite de ce chapitre, nous présenterons une recherche-action menée par le Laboratoire d'étude et de recherche sur l'intervention sociale (Léris) ${ }^{64}$ et le réseau Initiatives pour une agriculture citoyenne et territoriale (InPACT) en Occitanie depuis 2019, intitulée «L'alimentation des personnes en situation de précarité : de l'assignation à l'émancipation. Co-construire des tiers-lieux de solidarité et de transition alimentaire».

Ce projet, conçu selon les principes de la recherche-action dite coopérative, s'intéresse simultanément à plusieurs dimensions de l'alimentation (sociale, environnementale, économique, politique notamment), et adopte une démarche de recherche «à partir de l'action» et «en vue de l'action» (Bonny, 2014). Il met l'accent sur les enjeux démocratiques de l'accès à l'alimentation (Scherer, 2018).

Sur le principe d'une recherche à partir de l'action, elle vise à observer, analyser et prendre en compte les dimensions suivantes, à l'échelle territoriale :

- les pratiques alimentaires des personnes en situation de précarité et à petit budget : modes et lieux d'approvisionnement, choix et possibilités de consommation (en lien avec les enjeux de budget, de santé, de culture...), fabrication des repas et équipement disponibles, socialisation autour de l'alimentation;

- les pratiques et dispositifs de solidarité alimentaire : pratiques professionnelles et bénévoles d'intervention sociale en lien avec l'accès à l'alimentation, types de produits proposés et modes d'approvisionnement des lieux de solidarité alimentaire, dispositifs publics et privés pour fournir et distribuer l'alimentation, pratiques d'entraide informelle;

- l'identification des politiques publiques alimentaires et d'action sociale, et leur potentiel rapprochement;

- l'identification des acteurs composant les systèmes alimentaires territorialisés $(\mathrm{SAT})^{65}$, notamment en termes de production.

61. Solidarité en primeur(s) : https://solenprimcom.files.wordpress.com (dernière consultation le 30/08/2021).

62. www.civam.org (dernière consultation le 30/08/2021).

63. https://projetfrugal.fr/ (dernière consultation le 30/08/2021).

64. Le Léris est une association loi 1901 qui regroupe des chercheurs en sciences sociales engagés dans des démarches de recherche-action et des travaux d'étude : www.leris.org (dernière consultation le 30/08/2021).

65. Un SAT est «ensemble de filières agroalimentaires répondant aux critères du développement durable, localisées dans un espace géographique de dimension régionale et coordonnées par une gouvernance territoriale» (Rastoin, 2016, p. 13). 
Cette recherche-action ne met pas l'accent sur une observation quantitative des pratiques sociales liées à l'alimentation. En cela, ses résultats ne sont pas généralisables à l'échelle d'une population plus grande selon un principe de représentativité statistique. Elle s'appuie plutôt sur les données existantes dans la littérature, qu'elle met en résonance avec les données qualitatives issues du terrain, ainsi qu'avec l'expression des personnes impliquées dans le processus et le partage de leurs expériences vécues et de leurs pratiques. Ce partage d'expériences constitue à la fois une base pour le processus d'action collective inhérent à la recherche-action coopérative et un socle de données pour le travail de recherche et la compréhension des processus de changement liés aux actions mises en place.

Sur le principe d'une recherche en vue de l'action, «c'est-à-dire se donnant explicitement pour visée de produire des connaissances directement en prise sur des finalités pratiques » (Bonny, 2014), elle vise à observer, analyser et outiller les mouvements ou changements suivants :

- la transformation des pratiques alimentaires vers plus de sécurité, de qualité, de durabilité;

- la progression de l'accès à l'alimentation durable pour «tous »;

- la transformation des pratiques de solidarité alimentaire vers des pratiques plus émancipatrices;

- la transformation des pratiques des acteurs du système alimentaire vers plus de coopérations territoriales au bénéfice de la durabilité;

- la politisation de l'alimentation et les dynamiques de démocratie alimentaire;

- la recherche d'articulation entre expériences des personnes concernées, initiatives citoyennes, acteurs associatifs et privés, et politiques publiques, dans une logique de co-construction de l'action publique.

Ces différentes questions sont travaillées à l'échelle des groupes engagés et des territoires d'expérimentations. Cette échelle «micro» ne renvoie pas à un manque d'envergure de la recherche. Au contraire, l'échelle «micro» permet d'accéder à toute l'intensité des changements de l'expérience produits par la recherche-action, ainsi qu'aux processus de multiplication et de dissémination. En cela, l'échelle «micro» permet d'opérer une «montée en latéralité», par opposition à une «montée en généralité » susceptible de se heurter «à des formes de théorisation ou d'idéologisation verticales et globalisantes» (Nicolas-Le Strat 2013). Les résultats d'un tel dispositif de recherche-action sont donc particulièrement situés, socialement parlant, sans pour autant que cela constitue un frein à leur valeur scientifique, qui dépendra surtout du dispositif de recherche mis en place.

\section{W Points de vigilance et limites de la recherche-action}

La recherche-action dite coopérative pose des questions de méthode et de posture du chercheur qui nécessitent un travail réflexif permanent. Tout d'abord, qui pose la question de départ? Et pourquoi? D'où émergent le questionnement de recherche et sa formulation, dans une dynamique qui concerne différentes parties prenantes : chercheurs, acteurs et personnes impliquées, personnes concernées, institution, etc.? Le risque est d'imposer une problématique «par le haut», parfois par effet d'opportunité, 
sans se poser la question de la manière dont les premiers concernés abordent le sujet, ce qui entrerait en contradiction avec l'esprit même de la recherche-action. Ce point de départ peut se révéler bloquant pour le processus de recherche s'il n'est pas explicité et discuté. L'enjeu est la co-construction d'une problématique à partir d'une thématique, via les contributions singulières des différentes parties prenantes, notamment les savoirs expérientiels, par exemple ceux des personnes en situation de précarité. Par ailleurs, la recherche-action repose sur les deux dynamiques distinctes à partir de et en vue de l'action, qui peuvent se trouver en tension, notamment par rapport au changement visé : jusqu'où le processus de recherche-action est-il porteur d'une intention de changement (au risque de l'instrumentalisation)? À qui appartient cette intention? Comment est-elle ou non partagée par les différentes parties prenantes? Comment s'articule-elle avec le processus de co-production des savoirs et des pratiques? Cela questionne la posture du chercheur et son périmètre d'intervention, dans un allerretour entre investissement dans l'action et distanciation vis-à-vis de l'action, ce qui implique un réajustement permanent du rôle du chercheur et son adaptation aux différents contextes. Le dispositif de recherche lui-même est questionné. Lorsque plusieurs chercheurs y participent, ils peuvent par exemple tenir chacun des rôles différents, les uns plus engagés dans l'action, les autres plus distanciés. La valeur scientifique des résultats est intimement liée à ces réajustements permanents, ainsi qu'à leur explicitation. Une autre question centrale est celle des statuts des parties prenantes réelles (participant physiquement à la recherche) et symboliques (qui n'y participent pas physiquement, mais qui sont convoqués par les participants) au sein de la recherche-action : quelle est, notamment, la capacité des uns et des autres, y compris les chercheurs, à réinterroger leurs savoirs, à identifier leurs apports et à reformuler les hypothèses tout au long du processus. Enfin, pour les «personnes concernées » participant à un processus de recherche-action, celui-ci peut se révéler très impliquant, voire «invasif», ce qui crée une asymétrie, notamment en termes de rémunération, vis-à-vis des autres parties prenantes. Le statut des «personnes concernées» est un point d'attention particulièrement important dans une recherche-action dite coopérative, puisqu'ils sont susceptibles d'être tour à tour bénéficiaires, acteurs, enquêtés et analystes, ce qui nécessite la mise en place de dispositifs permettant de supporter cette complexité et les changements de rôles. Se posera également la question de la répartition des bénéfices du dispositif : à qui «profite» le travail collectif? Qui en retire quoi? La question se pose autant sur le plan matériel que symbolique, politique et de reconnaissance (gains financiers, effet sur une trajectoire de vie, acquisition de nouvelles compétences, accès à un nouveau droit, un travail, reconnaissance sociale, évolution professionnelle, visibilité, etc.).

\section{- Contribution de la recherche-action à une approche holistique de l'alimentation}

La recherche-action dite coopérative étant fondamentalement ouverte à la multiplicité des rôles et des points de vue, elle l'est également à la pluridisciplinarité. Elle engage des processus de recherche à partir des personnes concernées et au sein de démarches d'expérimentations situées, susceptibles d'intéresser, de concerner et de recourir à différentes disciplines. L'accès pour tous à l'alimentation durable, par 
exemple, soulève des questions relatives aux freins économiques, aux conditions et pratiques de vie (logement, équipement, matériel, milieux de vie...), à la mobilité, aux paysages alimentaires de proximité, à l'accès à l'information (nutrition, saisonnalité, santé...), aux dimensions sociales et culturelles (niveau d'éducation, structure familiale, culture d'origine, normes et valeurs qui influent sur les goûts alimentaires...), aux compétences psycho-sociales (représentations, préférences...). Ces questions invitent, en fonction des ressources disponibles, à réunir sociologie, anthropologie, économie, géographie, nutrition, psycho-sociologie au sein de la recherche-action. De plus, les dispositifs de solidarité et de démocratie alimentaires, qui se situent à la croisée des initiatives citoyennes, de l'action associative et des politiques publiques, invitent à faire participer les sciences de gestion, les sciences économiques et les sciences politiques. Ainsi, de nombreux savoirs disciplinaires et leurs représentants peuvent être invités à participer à un processus de recherche-action sur l'accès à l'alimentation durable, ce processus permettant de produire des résultats appropriables par ces différentes disciplines. La dimension holistique s'impose en quelque sorte d'elle-même. Elle procède de l'objectif de co-production des savoirs avec les personnes concernées, dont l'expérience est elle-même multidimensionnelle, et non découpée par les disciplines : un dispositif de recherche-action bien conçu ne peut faire l'impasse sur la multi-dimensionnalité de ses questions et de son objet, et doit en tirer les conséquences en termes de pluridisciplinarité.

\section{" Exemples d'application}

La recherche-action «L'alimentation des personnes en situation de précarité : de l'assignation à l'émancipation. Co-construire des tiers-lieux de solidarité et de transition alimentaire», menée par le Léris et le réseau InPACT de 2019 à 2021 en Occitanie, s'est intéressé à six expérimentations collectives de dispositifs de solidarité alimentaire axés sur la qualité de l'alimentation et l'exercice de la citoyenneté. Ces dispositifs ont impliqué des personnes en situation de précarité, des bénévoles et des salariés d'associations. Un tiers-lieu alimentaire est défini comme un espace coopératif reposant sur le principe d'acteur-bénéficiaire et sur une mixité d'activités et de modes d'actions, relatifs aux quatre grandes activités du système alimentaire - produire, transformer, distribuer, consommer -, à celles de l'éducation populaire - conscientiser, agir ensemble, transformer - et à celles de l'intervention sociale - accueillir, mobiliser, impliquer, accompagner -.

La problématique de cette recherche-action est la suivante : «Comment la mise en place de tiers-lieux de solidarité et de transition alimentaire peut activer des nouvelles formes de relations sur un territoire, permettant la création de liens sociaux et de solidarité, le soutien aux productions agricoles locales et durables, l'accès des personnes en situation de précarité à une alimentation choisie et qualitative, et l'évolution des pratiques alimentaires de tous vers plus de durabilité ? Comment développer conjointement une approche territoriale du système alimentaire, et une démarche de développement social partant des premiers concernés, qui soient vectrices de reconnaissance et d'émancipation, et qui permettent d'articuler dynamique démocratique, justice sociale et durabilité ?» (Léris, 2019). 
Trois grandes hypothèses structurent le programme :

- le développement d'une approche territoriale, partenariale et systémique de l'accès à l'alimentation permet de créer de nouvelles opportunités d'accès à une alimentation choisie et qualitative pour les personnes précaires, dans la perspective du droit à l'alimentation;

- la mise en œuvre d'une intervention sociale basée sur le développement du pouvoir d'agir, notamment via le développement de savoirs et savoir-faire, favorise l'autonomie et l'émancipation en termes d'accès à l'alimentation;

- la mise en place de modèles d'organisation coopératifs favorise le développement de rapports sociaux égalitaires et solidaires, et de dynamiques de démocratie alimentaire.

L'équipe du Léris a construit un dispositif basé sur la mise en place et l'accompagnement d'un groupe-projet par territoire, des temps de rencontre et de réflexion entre les groupes et avec des chercheurs, et la mobilisation des méthodes des sciences sociales : observations, entretiens individuels et collectifs et organisation de forums destinés à la restitution publique. Tout au long du programme, les groupes-projet ont été invités à utiliser différents outils de production de connaissances, notamment des enquêtes participatives sur les pratiques et aspirations alimentaires auprès d'habitants du quartier, de personnes accueillies à l'aide alimentaire et d'agriculteurs locaux; des ateliers de cartographie sur les ressources du territoire mobilisables dans le projet; des ateliers sur le repérage des compétences des personnes impliquées et des compétences nécessaires aux tiers-lieux, des ateliers de co-conception du dispositif; des temps de travail dédié à la co-construction du projet, etc.

L'expérience de deux des groupes engagés dans la recherche-action nous permet de préciser et d'interroger la méthode. Le premier est un collectif d'habitants situé dans un quartier en partie prioritaire, caractérisé par les faibles ressources économiques de ses habitants, mais avec une mixité sociale. Ce collectif se mobilise pour créer un café-épicerie-cuisine solidaire autour de l'alimentation durable (produits en vrac, groupement d'achats de produits frais en circuits-courts et de proximité, promotion de pratiques alimentaires saines, cuisine collective, etc.) et de la convivialité, du lien social, de l'insertion. Le deuxième est un groupe de bénévoles, dont certains ont l'expérience de la précarité, d'une grande association de solidarité animant un lieu d'accueil social et d'épicerie solidaire, situé en centre-ville et accueillant des personnes en grande précarité. Le groupe s'est mobilisé pour faire évoluer son action vers ce qui est désigné comme «un accès digne à une alimentation de qualité pour tous » avec deux axes principaux : promouvoir et proposer une alimentation de qualité et permettre aux personnes en situation de précarité de s'impliquer dans les actions pour développer leur pouvoir d'agir.

Dans la première expérience, la dimension très collective du projet, liée à une mobilisation de proximité à l'échelle d'un quartier et aux différentes portes d'entrée proposées vers le projet et vers l'alimentation durable (café, groupement d'achats, cuisine, four à pain, etc.) a produit une forme d'organisation horizontale (répartie en commissions thématiques) et autonome, qui nécessite une forte implication des membres et leur confère un fort pouvoir décisionnel. Dans ce projet, l'élaboration de critères de qualité pour le choix des produits alimentaires (charte) a par exemple fait l'objet d'un long travail commun, engageant ainsi une dynamique de 
démocratie alimentaire. L’implication immédiate de personnes bénéficiant de la solidarité alimentaire, dès les premiers pas de l'association et notamment du groupement d'achats, a ancré la démarche dans une dynamique ascendante, qui permet de partir des besoins et des aspirations des personnes concernées. L'ouverture du projet à un public mixte (précaire et non précaire) fait vivre le principe d'acteur-bénéficiaire propre aux tiers-lieux, apportant une vision de la solidarité plus ancrée dans la réciprocité que dans le rapport aidant/aidé. La forte implication que demande un tel projet pour les habitants et l'absence de structure de soutien «institutionnalisée » ont fait apparaître des limites et rendu nécessaire une réflexion et des ajustements pour ancrer cette dynamique dans le temps, assurer une forme de régularité (au regard des problématiques des foyers précaires) sans essouffler les acteurs du projet.

Dans la deuxième expérience, des paniers solidaires - paniers hebdomadaires de fruits et légumes locaux proposés aux familles en situation de précarité - ont été mis en place comme solution pour pallier la fermeture temporaire du lieu ordinairement occupé par le groupe. Cette seconde expérience a été moins productive à ce jour en termes de dynamique de démocratie alimentaire et de développement de forme d'organisation collective et participative, puisqu'elle a maintenu une distribution alimentaire plus classique. En revanche, cette action a engagé un changement de pratiques pour l'association sous plusieurs aspects : la mise à disposition régulière de fruits et légumes de qualité a produit des changements de pratiques alimentaires chez les personnes accueillies (manger plus de légumes, découvrir et cuisiner de nouveaux produits, etc.); les liens créés avec le producteur et le lieu de production (visites, pique-niques, ateliers cuisine) ont développé la sensibilité et la prise de conscience de l'ensemble des participants (bénévoles et personnes accueillies) sur les enjeux agricoles et alimentaires; le dialogue engagé avec les personnes accueillies autour de la distribution des paniers a permis de mieux connaître leurs pratiques et besoins, favorisant la constitution d'un groupe de travail participatif pérenne, voué à devenir le groupe de pilotage du futur tiers-lieu.

Ces deux processus existaient déjà indépendamment de la recherche-action, mais se sont appuyés sur l'accompagnement qu'elle propose. Plusieurs membres de chacun des groupes ont participé aux espaces de réflexion mis en place par la rechercheaction, contribuant ainsi au travail de co-production de la recherche, au-delà de leur appartenance au «terrain observé».

Dans les deux cas, ce qui a été expérimenté et construit relève de plusieurs registres : les modes de sensibilisation et de mobilisation des habitants et des personnes concernées par la précarité autour de la question alimentaire; les choix d'actions (groupement d'achats, jardin partagé, cuisine de rue, four à pain mobile, épicerie avec double tarif, cantine participative, ateliers divers, repas etc.); les choix de produits alimentaires; la recherche de fournisseurs, la logistique, la tarification; les critères d'accès à l'alimentation solidaire; les modes d'organisation collective et de prise de décision (organisation horizontale, participation des personnes en situation de précarité, mise en place de commissions thématiques, groupe de pilotage, conseil d'administration collégial, etc.).

Le travail de recherche-action a permis d'outiller ce processus de conception collective et d'en identifier les enseignements. Ceux-ci portent à la fois sur les effets de ces processus du point de vue des différentes parties prenantes et sur une modélisation 
méthodologique pour la construction d'un tiers-lieu de solidarité et de transition alimentaire. Les résultats portent notamment sur ce qui permet de lever les freins à l'accès à l'alimentation de qualité durable : le «faire ensemble», la coopération multi-acteurs, le financement des denrées, les liens et les partenariats entre producteurs et consommateurs, le partage de connaissances, l'articulation entre dynamique de tiers-lieu et politiques publiques et la manière dont ces expériences locales interpellent et contribuent à l'élaboration de nouvelles politiques. Ils portent aussi sur l'utilité sociale de ces tiers-lieux en termes de sécurité alimentaire, de santé, de lien social, de cohésion sociale à l'échelle d'un quartier, d'insertion professionnelle, de soutien au développement d'une activité agricole, mais aussi de développement de savoirs et compétences, notamment les compétences liées à l'action collective et les connaissances du système alimentaire et de ses enjeux. Cette recherche-action, qui est toujours en cours, a ainsi permis de produire des résultats sur les questions de démocratie et de justice alimentaires.

Pour conclure ce chapitre, nous soulignerons plusieurs enseignements tirés des cas d'application présentés, en termes de méthodologie et de déontologie de la recherche-action. Elles conduisent tout d'abord à mettre l'accent sur sa dimension fortement contextualisée. Le contexte d'application influe sur le processus tout en «faisant bouger» la méthode. Le point de départ du processus est déterminant : qui le lance et à quel stade sont impliquées les personnes concernées ? La portée transformatrice, notamment, en dépend. Deux types de dynamiques se côtoient dans les projets de tiers-lieux : une dynamique participative centrée sur les personnes concernées, les bénévoles ou les habitants, ainsi que sur leur appropriation des enjeux liés à l'alimentation durable et leur capacité à agir collectivement; et une dynamique de conception et mise en œuvre de projets, portée par les structures associatives, notamment celles qui disposent de salariés, qui répondent à des appels à projet pour financer les actions et structurer le processus. Cette seconde dynamique, plus dépendante d'institutions existantes, de leurs temporalités et de leurs espaces, peut entrer en contradiction avec la dynamique participative ou communautaire. Si ce double mouvement est nécessaire à l'évolution des projets, il est important de rester vigilant à ce que la logique de projet ne prenne pas le pas sur la dynamique participative.

Un autre point de vigilance concerne l'équilibre entre la dynamique sociale, portée par le groupe-projet, et l'intervention des chercheurs. Ces derniers doivent trouver le bon rapport entre «faire», «laisser faire» et «faire avec», pour ne pas imposer un rythme qui serait celui de la recherche-action, de ses financements et de ses livrables, tout en accompagnant les expérimentations. Les chercheurs doivent aussiveiller à ne pas rendre leur présence indispensable, car celle-ci a vocation à s'effacer dans la majorité des cas. Le degré d'intervention se négocie avec les participants. Ces derniers sont parfois en attente d'une implication plus forte, notamment en termes de gestion du projet, ce qui peut générer des insatisfactions ou des incompréhensions. Cette problématique est commune dans les pratiques d'organisation communautaire à visée émancipatrice, dans lesquelles les organisateurs sont amenés à s'effacer progressivement au profit des personnes concernées et de leur appropriation des processus d'action et de décision. À plusieurs reprises, les places et rôles de chacun ont ainsi dû être nommés et clarifiés. Cette clarification est d'autant plus nécessaire dans les cas où le rôle du chercheur est 
tenu par un praticien de l'organisation communautaire. Un travail de transmission des méthodes et des outils s'avère aussi nécessaire à la pérennisation des démarches. Une trop forte implication du chercheur peut induire un attachement à la réussite du projet, ce qui peut compliquer son travail d'analyse. La confrontation régulière des points de vue des différents participants et leur contribution au travail d'analyse permettent d'éviter cet écueil. Cependant, tous les participants ne peuvent s'impliquer de la même façon dans ce travail. Cela peut notamment s'avérer compliqué pour les personnes les plus éloignées du travail intellectuel. L'animation de temps de co-production de savoirs au sein des groupes et au rythme de l'émergence des questionnements permet de favoriser l'implication de tous.

D'autres enseignements peuvent être tirés sur le plan d'une approche holistique de l'alimentation. Cette recherche-action a soulevé des questions de nutrition, de santé, de stratégies d'approvisionnement et de transformations de pratiques alimentaires. Le recrutement d'un stagiaire a permis de commencer à aborder ces questions. Des collaborations plus poussées avec des nutritionnistes sont envisageables pour approfondir leur traitement. Les résultats nourrissent par ailleurs l'approche critique de la participation et l'analyse des processus d'émancipation chères au Léris, et enrichissent les connaissances opérationnelles pour la création de tiers-lieux alimentaires, notamment autour des enjeux de cohésion et de mixité sociale. Les processus d'expérimentations ont aussi permis d'observer des dynamiques de démocratie alimentaire à une échelle micropolitique, celle des groupes engagés. Une observation prolongée à plus long terme permettrait d'analyser l'approfondissement de ces dynamiques, ainsi que leur éventuelle amplification et leur articulation avec les politiques publiques. L'approfondissement des observations est aussi souhaitable et envisageable pour mieux connaître les conditions d'émergence de systèmes alimentaires territorialisés durables et inclusifs. De nouvelles coopérations entre acteurs du système alimentaire territorialisé ont été favorisées par les expérimentations mises en place, qui ont par exemple offert un appui à l'installation ou au développement de projets agricoles. Mais les observations quant à la dynamique systémique engagée à l'échelle du territoire restent limitées. Une meilleure connaissance de cette dynamique systémique à l'échelle territoriale est nécessaire pour mieux comprendre les conditions d'une transition vers des systèmes alimentaires plus durables. Pour produire cette connaissance, la poursuite de programmes de recherche-action étendus à des initiatives plus nombreuses au sein du territoire s'avère nécessaire.

\section{" Références}

Bonny Y., 2014. La recherche-action et la question de l'institution. Forum. Actualisation de la recherche-action et pertinence de la praxéologie, 142, 15-24. [En ligne] https://halshs.archivesouvertes.fr/halshs-01638846 (dernière consultation le 30/08/2021).

Cousin O., Rui S., 2010. L'intervention sociologique. Histoire(s) et actualités d'une méthode, Rennes, Presses universitaires de Rennes, 180 p. (coll. Didact Sociologie).

Fraser N., 2014. Qu'est-ce que la justice sociale? Reconnaissance et redistribution. Paris, Édition La Découverte, 182 p., (coll. Sciences humaines et sociales).

Léris, 2019. L'alimentation des personnes en situation de précarité : de l'assignation à l'émancipation. Co-construire des tiers-lieux de solidarité et de transition alimentaire, Montpellier, Léris, 14 p. https://drapps-occitanie.fr/wp-content/uploads/2019/05/Recherche-action-LERIS-tiers-lieux.pdf (dernière consultation le 30/08/2021). 
Maurel C., 2010. Éducation populaire et puissance d'agir. Les processus culturels de l'émancipation, Paris, LHarmattan, 244 p. (coll. Le travail du social).

Morvan A., 2013. Recherche-action, in Casillo I., Fourniau J.-M., Neveu C., Lefebvre R., Blondiaux L., Salles D., Chateauraynaud F., Barbier R. (éd.), Dictionnaire critique et interdisciplinaire de la participation, Paris, GIS Démocratie et Participation. [En ligne] http://www.dicopart.fr/ $\mathrm{fr} / \mathrm{dico} / \mathrm{recherche-action} \mathrm{(dernière} \mathrm{consultation} \mathrm{le} \mathrm{30/08/2021).}$

Nicolas-Le Strat P., 2013. Quand la sociologie entre dans l'action. La recherche en situation d'expérimentation sociale, artistique ou politique, Rennes, Éditions du commun, 232 p.

Paturel D., Ndiaye P., 2020. Le droit à l'alimentation en démocratie, Nîmes, Champ social Éditions, 242 p. (coll. Acteurs sociaux).

Paturel D., Le Velly R., 2014. Des circuits courts pour l'aide alimentaire? Hybridation de régulations dans un marché expérimental en Languedoc-Roussillon. Review of Agricultural and Environmental Studies/Revue d'études en agriculture et environnement, 94 (4), 443-465. [En ligne] https://halshs. archives-ouvertes.fr/halshs-00932848/document (dernière consultation le 30/08/2021).

Rastoin J.-L., 2016. Les systèmes alimentaires territorialisés. Enjeux et stratégies de développement. Journal Resolis, 7, 12-15.

Scherer P., 2018. La solidarité alimentaire de proximité comme espace d'expérimentation démocratique et vecteur d'émancipation. Recherche-action au sein du Secours populaire français en Occitanie. Forum, 153, 28-34. [En ligne] https://www.cairn.info/revue-forum-2018-1-page-28.htm (dernière consultation le 30/08/2021).

Touraine A., 1978. La voix et le regard, Paris, Seuil, 309 p., (coll. Sociologie permanente). 



\title{
Chapitre 14 \\ Les ateliers-théâtre : rendre compte de l'expérience sensible de l'alimentation
}

\author{
ESTELLE FOURAT ${ }^{a}$ ET FRÉDÉRIQUE JANKOWSKI ${ }^{b}$ \\ ${ }^{a}$ MoISA, Université Montpellier, IRD, CIRAD, CIHEAM-IAMM, INRAE, Institut Agro, Montpellier, France. \\ b CIRAD, UMR SENS, F-34398 Montpellier, France; SENS, CIRAD, IRD, Université Paul-Valery \\ Montpellier-III, Université Montpellier, Montpellier, France.
}

Les ateliers-théâtre engagent les participants dans une création artistique reposant sur des exercices d'improvisation encadrés, laissant une large part à l'expression d'émotions, d'expériences vécues et à la formulation de critiques et d'alternatives. La méthode exige, de la part des enquêteurs, des compétences de comédiens-animateurs et une sensibilité aux questions d'exclusion et d'oppression.

Le théâtre-action voit le jour en Belgique francophone dans les années 1970. C'est à la suite des revendications associées à 1968 qu'il constitue un cadre d'expression alternatif à une culture dominante (Biot, 1996; Brahy, 2019). Outil privilégié des acteurs sociaux, les «ateliers-théâtre » qui en découlent deviennent ${ }^{66}$ alors un dispositif singulier visant à renforcer la capacité créative et l'implication dans les débats de société de personnes socialement ou culturellement défavorisées. Appartenant à la grande catégorie des théâtres populaires ${ }^{67}$, le théâtre-action s'apparente dans le monde de la recherche aux méthodes qualitatives artistiques et participatives. Dans ce cadre, la mobilisation de cette approche répond généralement à des enjeux de production de connaissances, notamment dans leur dimension sensible (c'est-à-dire vécue et expérientielle), et des enjeux opérationnels, de transformation de situations considérées comme injustes ou non souhaitables du point de vue des parties prenantes. Son usage est encore rare dans les recherches en alimentation.

Dans un premier temps, ce chapitre revient plus généralement sur l'usage du théâtre dans la recherche et ses spécificités en tant que méthode participative et sensible aux expériences vécues et affects des individus ${ }^{68}$. Dans un second temps, nous décrivons

66. Selon l'arrêté belge du gouvernement de la Communauté française relatif au théâtre-action (2005).

67. En tant que mouvement artistique et politique d'un théâtre pensé pour et par le peuple, répondant à des enjeux de démocratie culturelle.

68. Dans le sens où le théâtre, plus encore que d'autres méthodes d'enquêtes qualitatives (entretiens, focus groups, observations ethnographiques par exemple), permet de susciter, d'extérioriser et de communiquer des sensations et des affects par le biais du jeu et de la prise de rôle. C'est dans ce sens que le qualificatif «sensible» sera utilisé dans la suite du chapitre. 
la manière dont un dispositif de théâtre-action permet de libérer la parole et de révéler les injustices sociales telles que vécues par les individus. Mis au service d'une problématique de recherche ${ }^{69}$ concernant les modalités d'inclusion sociale et culturelle d'un supermarché coopératif en Belgique, des ateliers-théâtre ont rendu visibles les mécanismes sous-jacents aux inégalités d'accès à une alimentation de qualité, et plus précisément les sentiments d'injustice associés au fonctionnement de la structure. Cette enquête sert de cas d'étude pour illustrer l'originalité de la méthode, ainsi que ses apports pour des recherches en alimentation.

\section{" Descriptif et apports de la méthode des ateliers-théâtre}

\section{Des usages du théâtre dans la recherche participative}

Les formes théâtrales de la famille du théâtre populaire englobent un grand nombre d'appellations et de formats (théâtre social, théâtre communautaire, théâtre pour le développement, théâtre de l'opprimé, théâtre appliqué, etc.), de pratiques et d'arrangements pratiques divers (Conrad, 2004). Ces différentes formes partagent le fait d'engager une diversité d'acteurs dans la création théâtrale. Cette dernière est considérée comme un moyen d'expression, mais également d'analyse réflexive et d'exploration de pistes d'émancipation, voire de transformations personnelles et sociales. Augusto Boal (1996), fondateur brésilien du théâtre de l'opprimé, met en avant la portée transformatrice du théâtre, qu'il définit comme une forme de connaissance permettant de construire notre avenir.

Dans la recherche scientifique, la mobilisation de formes collaboratives de théâtre répond à ce double enjeu cognitif et transformationnel. Ces démarches sont issues des sciences participatives et de l'ethnographie de la performance ou «art-based» ethnographie (Conrad, 2004; Dennis, 2009; Muller et al., 2017). Elles reposent toutes sur l'analyse d'autoreprésentations, c'est-à-dire de représentations de la réalité créées par les acteurs concernés. La première grande distinction avec les méthodes qualitatives classiques est que l'interprétation de ces autoreprésentations s'inscrit dans un processus de négociation de significations dans lequel le chercheur n'est pas le seul interprète de la représentation, mais partage ce rôle avec les différents participants dans le cadre d'une analyse collective. La deuxième distinction majeure est que de telles approches se donnent comme finalité d'offrir des «prises » aux acteurs pour qu'ils puissent agir sur leur monde. Ces démarches répondent donc à des enjeux éthiques et politiques de la recherche (Jankowski et al., 2020).

Le théâtre en tant que méthode de recherche participative est mobilisé par diverses disciplines, comme la sociologie, l'ethnologie, la psychologie, la médecine, les sciences de l'éducation et de l'environnement (pour une synthèse : Heras et Tàbara, 2014). Cependant, le niveau d'engagement réel des individus et le mode de participation peuvent varier considérablement. Deux grandes approches peuvent être identifiées :

69. Recherche Falcoop (Favoriser l'accès à une alimentation durable et de qualité pour un public représentatif de la diversité urbaine par l'implantation locale d'un modèle innovant de supermarché coopératif), financée par Innoviris. 
celle où les expériences personnelles des participants constituent la base de la création théâtrale, comme c'est le cas pour les ateliers-théâtre du théâtre-action; et celle où un spectacle est créé par une équipe d'animateurs, puis joué devant un public ciblé, qui participe ensuite à un forum au sujet de la pièce (Heras et Tabara, 2014). Dans tous les cas, pour les chercheurs mobilisant le théâtre comme méthode d'investigation, la pratique théâtrale est considérée comme une forme de connaissance par l'implication tant des esprits que des corps. Certains auteurs soulignent le rôle clef de l'action dans le théâtre, qui permet l'expression de savoirs incarnés, culturellement situés et socialement distribués. Selon Courtney, la pratique théâtrale «offre une autre façon d'acquérir des connaissances - une façon unique et puissante d'accéder aux connaissances, en tirant des réponses qui sont spontanées, intuitives, tacites, expérientielles, incarnées ou affectives, plutôt que simplement cognitives » (Courtney, 1988 in Conrad, 2004, p. 16). Les recherches qui utilisent un dispositif théâtral comme méthode s'inscrivent ainsi dans des approches à la fois participatives et sensibles, en réintroduisant la dimension affective au cour de la production de connaissances.

\section{Les ateliers-théâtre}

C'est spécifiquement sur ces dimensions expérientielles, incarnées et affectives que les ateliers-théâtre définis par le théâtre-action s'appuient pour réaliser une «création collective». Brahy (2014) qualifie le mode d'engagement des participants à ces ateliers d' «engagement en présence». Ce dernier développe des modalités de coordination nouvelles entre les participants qui «font la part belle aux émotions, au ressenti et aux corporéités»(Brahy, 2014, p. 46). Les ateliers-théâtre impliquent des personnes «ordinaires » qui sont guidées par des «comédiens-animateurs » pour réaliser une création collective qui met en scène des dimensions de leurs vécus. «Très concrètement, l'atelier-théâtre est une activité composée d'un nombre de séances répétées (généralement 3 heures, une fois par semaine) avec un même groupe (généralement composé d'une douzaine de personnes, variant de deux participants à une petite vingtaine au maximum) pendant une durée relativement longue ou intense (généralement 10 mois) permettant d'envisager sérieusement (sans toujours y parvenir) une création collective (le plus souvent théâtrale), où les participants sont les acteurs de la pièce» (Brahy, 2011, p. 80-81).

Une séance est constituée d'exercices d'improvisation qui ont pour vocation de développer l'aisance expressive des participants et une certaine confiance vis-à-vis du groupe au travers de langages expérimentés collectivement.

Le langage peut être verbal, comme dans l'exercice du «gromolo». Celui-ci correspond à un langage sonore inventé, recourant à de nombreuses onomatopées (par exemple : Ooh! Aah! Blarg!). Ce langage en lui-même n'a pas de sens : ce sont les gestes, le volume et les intonations de la voix qui permettent aux participants de communiquer entre eux. Cette technique a pour objectif de porter l'attention sur l'énergie qui accompagne l'énoncé et de se détacher de la recherche du mot juste. Un tel langage permet aussi de se défaire des discours préconstruits pour faire porter l'échange sur l'émotion, le ressenti de la personne.

Le langage peut être également corporel, comme dans l'usage des «statues». Dans cet exercice, les individus doivent s'immobiliser dans une posture particulière qui 
représente ce qu'une situation, un moment ou un terme évoque en eux. La notion de statue renvoie donc à une posture psychologique particulière. Dans ce cas, le signifiant (le corps) et les signifiés (les mots, les images) sont considérés comme inséparables. Même figées, les statues sont évocatrices d'une émotion : la joie, la peur, la tristesse, la colère, etc.

\section{Les apports (et contraintes) en tant que méthode participative et sensible}

Cette approche en tant que méthode d'enquête se distingue des entretiens individuels semi-directifs et des entretiens collectifs en plusieurs points :

- les postures et la relation de l'enquêteur et des enquêtés se distinguent clairement de celles adoptées généralement dans les entretiens classiques. À la différence d'un entretien semi-directif ou d'un entretien collectif, la pratique théâtrale, par ses techniques d'improvisation, permet de rompre avec les rapports hiérarchiques en établissant une équivalence de statut entre participants;

- au travers d'exercices d'improvisation et de la création collective de la pièce de théâtre, des autoreprésentations, c'est-à-dire des représentations de la réalité créées par les acteurs concernés, sont affirmées, analysées et critiquées par le groupe luimême - et non uniquement par le chercheur - lors des séances.

- les ateliers-théâtre ne s'inscrivent pas dans des formes délibératives classiques. Ils autorisent le dépassement des difficultés associées au langage comme la construction d'un discours articulé au travers de codes expressifs ordinaires. En effet, les exercices mobilisés prennent appui sur une diversité de modalités expressives verbales et non verbales (dessins, exercices corporels, langage simplifié et inventé tel que le «gromolo » cité plus haut, etc.), qui facilitent l'expression et la libération d'émotions face à un groupe, a fortiori lorsqu'il s'agit d'une langue étrangère pour les acteurs; - si l'explicitation des différents points de vue est recherchée dans les entretiens collectifs (tels que les focus groups) comme dans les ateliers-théâtre, les premiers produisent davantage des processus de régulation/temporisation des émotions, mis en œuvre tant par l'enquêteur que par les enquêtés, soit pour permettre à chacun des participants d'expliciter son point de vue, soit pour rendre celui-ci plus «objectif». Or, dans le cas des ateliers-théâtre, la diversité constitue également le support d'une expression individuelle sensible, qui prend appui sur une rythmique collective portée par une émotion qui n'est plus contrainte par le collectif;

- de même, contrairement au cadre des entretiens collectifs, dont l'un des enjeux est la définition d'un discours collectif a posteriori, dans les ateliers-théâtre ce dernier est un enjeu partagé par l'ensemble des participants au travers de la création collective. Ce discours collectif prend la forme de scénettes créées par l'ensemble des participants. Ce discours collectif peut parfois relever de figures de style artistiques singulières comme le registre métaphorique;

- le théâtre permet également d'ouvrir des espaces de débat à un collectif élargi, celui des publics des représentations théâtrales. Le recours aux métaphores et symboliques participe du partage des émotions avec le public. Ces procédés définissent le cadre d'une relation empathique entre les comédiens et les spectateurs. Ainsi que nous le verrons, ils offrent également le cadre d'une argumentation critique sensible ancrée dans les vécus des acteurs; 
- le débat qui a lieu après la présentation de la pièce permet l'explicitation des différents points de vue, parfois contradictoires. Les divergences de perspectives exprimées peuvent être considérées au travers d'un objectif partagé entre l'ensemble des individus : la définition commune de solutions alternatives aux tensions représentées. Ces débats produisent un matériau original d'analyse sur les régimes de justification mobilisés par la diversité des acteurs en présence et l'observation des processus de co-construction d'alternatives considérées comme justes par tous;

- enfin, par rapport à d'autres méthodes participatives qui font face à la difficulté de la participation dans la durée des participants, l'atelier-théâtre favorise l'engagement des individus par le plaisir partagé de «faire commun» et l'engagement mutuel autour d'une démarche de création collective;

- cependant, cette méthode présente des contraintes. Il ne s'agit pas d'une méthode d'enquête «rapide» facile à déployer à l'improviste. Elle implique de travailler avec un professionnel moyennant un coût, et nécessite l'implication des participants durant plusieurs mois tout au long de la création collective et des représentations.

Cette démarche d'enquête collective accède à des dimensions que des méthodes classiques d'enquête peinent à saisir, comme l'explicitation des dimensions sensibles de la connaissance. Dans le cadre d'études portant sur les représentations et pratiques alimentaires, l'usage du théâtre, au travers du registre des émotions, crée un espace propice à l'expression et au dialogue sur les difficultés et injustices vécues, ce qui sera illustré dans la section suivante («Une enquête sur l'inclusion sociale au sein d'un supermarché coopératif»). Ces injustices vécues peuvent être liées aux sentiments d'incapacité à se conformer à des discours normatifs (manger bio ou manger sain par exemple), à des sentiments d'inégalités d'accès à une alimentation désirée, ou encore à des sentiments d'exaspération vis-à-vis d'une situation sociale vécue comme pénible.

La méthode théâtrale nourrit aussi une approche holistique du fait alimentaire. Articulée à des méthodes plus classiques d'enquête, elle permet de réintégrer dans la recherche les affects des individus, formulés au travers de l'explicitation d'injustices vécues, portées collectivement. Au-delà de la simple identification d'un problème ou de son analyse collective à huis clos, elle permet sa mise en débat lors de représentations publiques. Selon les objectifs visés et le dispositif théâtral mobilisé pour y répondre, ces débats peuvent prendre la forme de véritables expérimentations pour la définition d'actions collectives. Elle est ainsi une pratique transformatrice et émancipatrice pour le groupe, dans la mesure où les processus de création et de représentation publique participent de l'acquisition et du partage de nouveaux savoirs et savoir-faire pour les participants. En tant qu'approche performative, la méthode a donc des effets sur l'existant des acteurs : elle peut par exemple renforcer, au travers de son évocation collective, le sentiment d'injustice des individus. Pour les chercheurs mobilisant cette démarche, il est donc nécessaire d'être conscients et vigilants sur les effets que le processus performatif peut produire tant sur les individus que sur les collectifs.

Plus largement, l'approche théâtrale peut également offrir le cadre d'un dialogue transdisciplinaire. Au travers d'un langage imposé à tous, celui de la représentation scénique, elle implique un ensemble de traductions et d'explicitations des cadres interprétatifs mobilisés par chacun des participants (académiques ou non académiques). Elle peut permettre de représenter, de partager et de mettre en débat des résultats issus de la recherche, mais également les procédés (facteurs, 
hypothèses) les ayant produits ${ }^{70}$ (Faye et al., 2018). Dans une perspective intégrative $^{71}$, la création théâtrale peut aussi être conçue comme la modélisation d'une situation ou d'un fait en donnant à voir, dans un espace (la scène) et un temps limité (celui des représentations théâtrales), les interrelations entre différentes dimensions et leurs effets (Jankowski, 2019; Jankowski et al., 2016).

La mobilisation d'un dispositif théâtral comme méthode d'enquête peut donc répondre à une diversité d'objectifs, être pensée en articulation avec d'autres méthodes d'enquêtes qualitatives ou dans une approche transdisciplinaire. Selon les enjeux portés par la recherche, le dispositif théâtral et les modalités de sa mise en œuvre doivent donc être clairement définis en amont de la recherche.

\section{" Une enquête sur l'inclusion sociale au sein d'un supermarché coopératif}

Dans la recherche-action Falcoop ${ }^{72}$, le théâtre a été convoqué comme outil de libération de la parole sur une réalité vécue et perçue singulière, celle de faire ses courses dans un supermarché coopératif et d'y consacrer trois heures mensuelles, pour une population non familiarisée à ce genre de magasins. Au travers d'ateliers-théâtre, il s'agissait plus spécifiquement de faire expliciter les représentations et les valeurs que des habitants du quartier associent à cette structure de distribution alimentaire; de mettre à l'épreuve les principes de justice socioculturelle prédéfinis par les coopérateurs du supermarché en les soumettant aux ressentis des habitants; et d'ouvrir des espaces de dialogue entre les habitants du quartier et les coopérateurs lors des représentations publiques pour renforcer l'objectif d'inclusion sociale et culturelle du supermarché. Dans les paragraphes qui suivent, nous revenons plus spécifiquement sur le contexte de l'enquête et la manière dont les ateliers-théâtre ont, d'une part, permis l'explicitation des affects associés au supermarché coopératif et, d'autre part, offert le cadre d'une critique métaphorique au sujet de son fonctionnement.

70. Dans le cadre d'une recherche sur la gestion des ressources hydriques au Sénégal, un modèle économique de l'évolution de la ressource a été scénarisé. Les différents facteurs considérés dans la modélisation ont été ainsi rendus explicites pour l'ensemble des autres acteurs du projet. Les forums ont fait apparaître la non-prise en compte de dimensions d'importance. Celles-ci ont été alors réintroduites dans la modélisation économique.

71. Dans le cadre de recherches sur la modernisation du pastoralisme au Sahel, sur la circulation des ressources phytogénétiques ou encore la gestion concertée de territoires, des dispositifs de théâtre ont été développés avec des chercheurs relevant de différentes disciplines comme la socio-anthropologie, l'économie, la géographie, les sciences politiques, la zootechnie, les sciences environnementales ou les sciences génétiques.

72. Le projet de recherche-action a proposé d'accompagner la mise en pratique des objectifs d'inclusion sociale et culturelle d'un supermarché coopératif de Bruxelles. Le modèle du supermarché coopératif repose sur la participation bénévole - un «shift», tel que nommé par la coopérative - de 2 heures 45 mensuelles de membres à la fois actionnaires (part minimale de 25 euros) et détenteurs d'une carte de membre. Seuls les détenteurs d'une carte de membre ont accès au magasin pour faire leurs courses (il faut montrer sa carte à un membre qui est posté à l'entrée du magasin, une borne magnétique sera ensuite installée) et participer à la gouvernance lors d'assemblées générales. L'accessibilité à tous, indépendamment de l'âge, du sexe, de la nationalité, de la langue ou encore des moyens financiers des consommateurs, est ce qui a motivé la création de ce supermarché coopératif. 


\section{Adaptation des ateliers-théâtre au contexte de l'enquête}

La définition du collectif de "participants-acteurs» de l'atelier-théâtre a été réalisée en concertation entre une chercheuse du projet, une bénévole du supermarché coopératif et une animatrice d'une association d'éducation permanente, historiquement destinée aux populations turques immigrées et située dans le même quartier. Le collectif regroupait, au total, une dizaine de personnes, présentant une diversité de nationalités (deux Belges, une Française ${ }^{73}$, trois Turques, deux Marocains, une Arménienne et un Algérien), âgés de 30 à 50 ans, et une diversité de pratiques alimentaires. Par exemple, les deux membres de la coopérative et l'animatrice du groupe avaient l'habitude de fréquenter des magasins biologiques ou des coopératives d'achat. Par contre, les membres de l'association n'avaient jamais adhéré à une telle coopérative alimentaire et fréquentaient habituellement les épiceries ethniques et les hard-discounters, dans lesquels ils achetaient exceptionnellement des marques «bio». Lors de la première rencontre avec les personnes volontaires, il s'est avéré qu'elles avaient un niveau de français très faible. Dans ce contexte, les exercices d'expression visuelle et corporelle mobilisés par la «comédienneanimatrice» des ateliers-théâtre ont permis de dépasser les limites linguistiques et favorisé le dialogue entre tous. Le choix de ces exercices, ainsi que le format final de la représentation de la création collective ont été conditionnés par cette contrainte linguistique. Ainsi, à chaque séance, divers exercices d'expression gestuelle et vocale étaient proposés aux participants pour développer leur aisance expressive et une certaine confiance vis-à-vis du groupe. Un ressort important des ateliers-théâtre est ainsi la création d'un sentiment d'appartenance collective au travers de l'expérience commune (Brahy et Servais, 2016).

\section{Le supermarché coopératif au prisme des affects}

Pour que les participants puissent exprimer leurs émotions, la comédienne-animatrice a mobilisé différentes techniques d'improvisation qui n'étaient pas nécessairement verbales comme cela peut être le cas dans un groupe de discussion classique. Certains exercices ont ainsi porté sur le ressenti corporel de l'être-ensemble au travers d'une coordination motrice et d'un engagement rythmique corporel collectif. Par exemple, au cours de l'une des rencontres, la comédienne-animatrice a invité l'ensemble des participants à se mettre en ligne, épaule contre épaule. La consigne était d'avancer ensemble au même rythme, en maintenant le contact et en disant chacun une phrase commençant par «j'en ai marre de...». Cet énoncé devait s'accompagner d'un sentiment de colère croissant au fil de la progression sur la scène. Après avoir répété plusieurs fois ce déplacement collectif, la comédienne-animatrice a proposé le même exercice d'improvisation, mais en demandant aux participants de penser à la coopérative sans la nommer; en se référant davantage à quelque chose qui la représente, qui touche à l'alimentation ou à la nourriture qui y est associée du point de vue des participants. Dans cet exercice, une même émotion - la «colère », selon la consigne explicitement donnée aux participants, anime l'ensemble des participants.

73. N'étant pas animatrice de théâtre, la chercheuse impliquée dans la démarche a été intégrée au processus créatif par une posture d'observation participante maintenue sur le long terme. 
Selon les individus, elle est associée à différentes dimensions du supermarché coopératif. Ainsi, en cœur, nous pouvions entendre :

«'en ai marre des cartes!

J'en ai marre du magasin de travail!

J'en ai marre des prix trop chers!

J'en ai marre du mot "bio", "bio", "bio"!

J'en ai marre que tout ce qui est bon [soit] trop cher!

J'en ai marre du bio!

J'en ai marre des magasins bios!

J'en ai marre qu'on nous bassine à nous dire qu'il faut manger bio !

J'en ai marre qu'on ne fasse rien concrètement!»

Dans cet exercice, l'émotion commune à tous les participants a été utilisée comme vecteur de l'expression individuelle.

\section{Le cadre d'une critique métaphorique}

Il nous semble intéressant de souligner l'importance des références métaphoriques et symboliques mobilisées dans cette création collective et, notamment, leur rôle dans la construction d'un discours critique sur le fonctionnement du supermarché coopératif et dans la mise en partage des émotions. Au fil des ateliers-théâtre une pièce composée de trois scènes a été co-construite.

La première scène de la création collective s'appuie sur la métaphore du voyage en fusée pour rejoindre une autre planète : le supermarché coopératif. La fusée figure ici un voyage pour rejoindre un espace inconnu, distant de soi. Les passagers expriment le fait de ne pas comprendre pourquoi ils sont amenés à faire ce voyage vers une planète qui ne les concerne pas vraiment. Cette scène représente également un «shift» (échange) entre membres de l'équipage aux rôles et compétences très différents : d'une part, l'hôtesse de l'air et d'autre part, le commandant de bord. Les passagers sont spectateurs de ce changement de fonction surprenant de leur point de vue et sont soumis à des messages qu'ils ont du mal à saisir. Cette scène exprime ainsi les incompréhensions multiples ressenties par les participants du projet par rapport à une structure qu'ils ne considèrent pas leur être destinés; l'interchangeabilité des membres de la coopérative dans les différentes tâches à accomplir au sein de celle-ci et; les messages perçus comme des injonctions qui y sont associées.

La deuxième scène mobilise la métaphore de la douane pour représenter l'accès limité au supermarché coopératif et le symbole des cartes comme modalité excluante. La carte du supermarché est classée par les participants dans la même catégorie que les cartes bancaires, les cartes de crédit, etc. Cette métaphore procède de l'explicitation de catégorisation opérée par les participants pour donner sens à une dimension du fonctionnement de la structure coopérative : son accès contrôlé sur la base d'un statut de membre. Dans cette scène, la douanière semble être plus flexible au sujet des règles lorsqu'on lui présente des cartes-symboles de la consommation ou que lui sont tendus des billets. Cette scène inscrit ainsi le supermarché coopératif dans un modèle consumériste plus global qui demeure excluant puisqu'il 
exige une carte de membre, générant de cette manière des mécanismes de sélection et donc de renforcement des inégalités.

La dernière scène repose sur la métaphore de la découverte d'une nouvelle planète, sa nourriture et ses habitants. Tout y semble étrange et surtout particulièrement cher. Les habitudes des habitants de cette planète apparaissent irrationnelles pour les voyageurs. Les participants du projet expriment ainsi, une nouvelle fois, leur incompréhension face à la cherté des produits vendus par le supermarché coopératif et à la logique sous-jacente à son fonctionnement participatif.

Au-delà de sa dimension critique, le recours à la métaphore opère un déplacement symbolique qui contribue à la mise en commun des vécus. La métaphore participe généralement d'une projection de la structure d'un domaine d'expérience particulier sur un autre domaine. Dans la création collective, la métaphore du voyage vers une planète lointaine et étrange a pour vocation d'interpeller les spectateurs au travers d'une expérience commune. Le voyage constitue, en effet, un domaine d'expérience cognitivement saillant, facilement mobilisable et qui permet ici de saisir un autre domaine d'expérience plus abstrait pour des spectateurs aux histoires territoriales hétérogènes. La métaphore participe ainsi d'une réorganisation du champ interprétatif et conceptuel de la situation. Elle permet de reconsidérer la valeur du concept «supermarché coopératif» au regard des parcours et des usages des participants. En d'autres termes, la théâtralisation métaphorique permet aux participants d'exprimer non pas uniquement leurs perceptions du supermarché coopératif, mais aussi de partager avec les spectateurs ce que ce supermarché suscite en eux, l'expérience et les sentiments auxquels il fait écho. Les sentiments d'étrangeté et d'exclusion éprouvés par les participants sont associés à ceux qu'ils peuvent ressentir plus globalement dans leur société d'accueil en tant que migrants. La référence à l'expérience personnelle et à une forme d'intimité du vécu permet ainsi l'inscription de la critique portée sur le mode de fonctionnement du supermarché coopératif dans un registre argumentatif sensible.

En conclusion, ce chapitre décrit certains des apports et des contraintes des ateliersthéâtre comme méthode d'enquête participative et sensible, illustrés par un cas d'étude sur l'inclusion sociale d'un supermarché coopératif à Bruxelles. Au travers de la description de l'un des exercices d'improvisation théâtrale et du recours à la métaphore dans la création collective, il s'agissait de rendre compte des ressorts singuliers que mobilise cette démarche (par rapport à d'autres plus classiques) pour l'explicitation et la mise en commun des représentations et affects associés à la structure coopérative. Nous y décrivons également la portée des métaphores mobilisées dans la création collective dans le partage des émotions avec un collectif élargi lors des représentations théâtrales. L'ensemble des métaphores associées au supermarché - le voyage vers un pays lointain, l'entrée par un passage à la douane, la nécessité de disposer de papiers, le coût de la vie - constituent aussi le supermarché comme une métaphore de la trajectoire de migration et du pays d'accueil. Le processus de création collective a ainsi permis aux participants de s'exprimer face aux publics non pas uniquement sur leur vécu des supermarchés coopératifs, mais depuis leur vécu d'immigrant au sujet de ces structures. Les critiques énoncées par les participants sur le fonctionnement du supermarché coopératif se trouvent intimement liées à leurs parcours et sont rendues visibles comme telles à d'autres acteurs. 
Comme nous le mentionnons en présentant la méthode, l'un des objectifs des démarches de théâtre-action est d'offrir des prises aux participants pour renforcer leur pouvoir d'action. Si cette expérience n'a pas modifié de manière profonde les représentations qu'ont les participants du supermarché coopératif, elle leur a offert un espace d'expression et de légitimation des critiques qu'ils adressent à la structure coopérative. Cette création collective a été présentée à plusieurs reprises face à un public composé de coopérateurs du supermarché coopératif, travailleurs sociaux ou de l'animation culturelle, chercheurs, familles des acteurs. Face au discours critique dressé au travers de la pièce à l'encontre du supermarché coopératif, deux réponses de nature opposée ont été exprimées par les spectateurs. Certains ont accueilli cette critique de manière constructive comme un moyen de faire évoluer la structure et son fonctionnement. D'autres, par contre, ont exprimé leurs désaccords par rapport à la critique (jugée comme résultant d'un manque d'information), sur l'obligation de travailler (alors que c'est un engagement social), les prix trop élevés (alors qu'ils se justifient par la juste redistribution aux producteurs et à la qualité des aliments) ou encore l'injonction au «bio » mise en avant dans la pièce. Le débat a ainsi permis de rendre public un clivage existant au sein du supermarché et mis à l'épreuve les principes de justice affichés. La question de l'accès à tous au supermarché coopératif fait, effectivement, l'objet de débats lors des assemblées : débats qui se cristallisent généralement autour de la nature des aliments à proposer comme, par exemple, le fait de vendre ou pas de la viande halal. De la même manière que les espaces de représentations théâtrales ont permis de rendre publics les sentiments d'injustice éprouvés par les participants, ils ont aussi donné à partager plus largement des débats normalement internes à la structure. Les principes de justice socioculturelle affichés par le supermarché se retrouvent questionnés par la pluralité des vécus, des conceptions du «bio» et des justifications concernant son prix.

Mais l'enjeu principal ici était celui de l'explicitation et la prise en compte des points de vue des habitants immigrés et non familiers de ce genre de structure alimentaire, et moins une démarche multi-acteurs (impliquant la diversité des types d'acteurs concernés) pour la recherche de nouvelles modalités de gouvernance. En ce sens, il ne s'agit pas d'une démarche de théâtre forum par exemple, dont l'un des enjeux principaux est l'expérimentation et la définition collective de nouvelles modalités d'action au travers des forums. Depuis, des éléments de fonctionnement du supermarché coopératif ont été tout de même débattus et ont évolué avec la mise en place, par exemple, d'un système solidaire de partage du temps de bénévolat. D'autres semblent difficilement modifiables, comme attribuer des marges réduites à des aliments de première nécessité (tels que la farine, les céréales, l'huile) pour les rendre plus accessibles financièrement (Fourat et al., 2020).

Ainsi que nous l'évoquons, la mobilisation de formes théâtrales comme méthodes d'enquête implique un ajustement de la démarche au contexte et aux objectifs de recherche. Il ne s'agit nullement d'une «méthode-outil» prête à être utilisée quels que soient les enjeux de la recherche. Elle implique un engagement important des participants (dont les chercheurs font partie) et une diversité d'expertises (thématiques, théâtrales, expérientielles). En tant que méthode de recherche, elle offre par ailleurs un cadre inédit d'explicitation des affects engagés dans des contextes relationnels singuliers. 


\section{W Références}

Biot P. (éd.), 1996. Théâtre-action de 1985 a 1995. Itinéraires, regards, convergences, Cuesmes, Édition du Cerisier, 464 p.

Brahy R., 2011. Le politique a-t-il déserté le théâtre-action? Mouvements, 65, 79-90. [En ligne] https ://www.cairn.info/revue-mouvements-2011-1-page-79.htm (dernière consultation le 30/08/2021).

Brahy R., 2014. L'engagement en présence : l'atelier de théâtre-action comme support à une participation sociale et politique? Lien social et politiques, 71, 31-49. https://doi.org/10.7202/1024737ar

Brahy R., 2019. S'engager dans un atelier-théâtre. Le sens de l'expérience, Cuesmes, Édition du Cerisier, 237 p. (coll. Place publique).

Brahy R., Servais V., 2016. Être avec par corps. L'expérience du commun sur un plateau de théâtre. Recherches en communication, 42, 141-153. https://doi.org/10.14428/rec.v42i42.48523

Boal A., 1996. Théâtre de l'opprimé, Paris, éditions La Découverte, 224 p. (coll. Poche/Essais).

Conrad D., 2004. Exploring Risky Youth Experiences: Popular Theatre as a Participatory, Performative Research Method. International Journal of Qualitative Methods, 3 (1), 12-25.

https://doi.org/10.1177/160940690400300102

Dennis B., 2009. Acting Up: Theater of the Oppressed as Critical Ethnography. International Journal of Qualitative Methods, 8 (2), 65-96. https://doi.org/10.1177/160940690900800208

Faye A., Jankowski F., Msangi S., Niemi Y., Fall C. S., Dia D., 2018. Combining quantitative modeling and forum-theatre to analyze agricultural water management options: the case of the Niayes region of Senegal. Sixth Annual International Conference on Sustainable Development (ICSD), 26-27 septembre 2018, Columbia University, New York.

Fourat E., Closson C., Holzemer L., Hudon M., 2020. Social inclusion in an alternative food network: values, practices and tensions. Journal of Rural Studies, 76, 49-57.

https://doi.org/10.1016/j.jrurstud.2020.03.009

Heras M., Tàbara J. D., 2014. Let's play transformations! Performative methods for sustainability. Sustainability Science, 9, 379-398. https://doi.org/10.1007/s11625-014-0245-9

Jankowski F., 2019. Le théâtre forum : rencontre transdisciplinaire. Séminaire Art et Science de l'IMéRA (Institut d'études avancées - Exploratoire interdisciplinaire Méditerranée), 21 janvier 2019, Marseille.

Jankowski F., Defives B., Diao Camara A., Botta A., 2016. Voipastorales : débattre des trajectoires des sociétés pastorales. Film 24'50. https://www.youtube.com/watch?v=fx30c9xjbfc\&feature=youtu.be

Jankowski F., Louafi S., Kane N. A, Diol M., Diao Camara A., Pham J.-L., Berthouly-Salazar C., Barnaud A., 2020. From texts to enacting practices: defining fair and equitable research principles for plant genetic resources in West Africa. Agriculture and Human Values, 37 (4), 1083-1094. https://doi.org/10.1007/s10460-020-10039-3

Muller B., Pasqualino C., Schneider A. (éd.), 2017. Le terrain comme mise en scène, Lyon, Presses universitaires de Lyon, 188 p. (coll. Nouvelles écritures de l'anthropologie, 5).

\section{Remerciements}

En premier lieu nous remercions les institutions qui ont soutenu le projet Falcoop : Innoviris (pour la recherche Falcoop/ULB), en partenariat avec la Maison de la Turquie (EYAD) dans le cadre de son programme d'éducation permanente, le supermarché coopératif la BEES coop et la Fédération des services sociaux (FdSS). Nous remercions également tous les participants des ateliers-théâtre pour leur générosité, engagement, et discussions qui ont donné lieu à cette création (Ani Arakelyan, Dilsen Dalyan, Otman Ebn Achehab, Gulsen Eskici, Jamie Lee Fossion, Wahid Ghermoul, Pascale Missenheim, Yuksel Tekinarslan), ainsi que l'animatrice de théâtre Brigitte Grisar pour la qualité de ses interventions ayant accompagné le processus de création. Nous sommes infiniment reconnaissantes envers Catherine Closson et Laurence Holzemer qui nous ont fait confiance pour tenter cette expérience théâtrale et pour les longs échanges sur cette recherche participative. Enfin, cette analyse est issue de la rencontre entre une chercheuse du projet Falcoop (Estelle Fourat) et une chercheuse travaillant depuis plusieurs années sur les usages 
du théâtre participatif dans la recherche (Frédérique Jankowski). Leur rencontre et ce projet de co-écriture ont été rendus possible grâce aux organisateurs du séminaire Méthodes d'investigation de l'alimentation et des mangeurs (MIAM), que nous remercions chaleureusement. 


\title{
Chapitre 15 \\ Les focus groups : étudier le fait alimentaire à travers des mises en discussion thématiques
}

\author{
AMANDINE ROCHEDY ${ }^{a}$ ET SANDRINE BARREY ${ }^{b}$ \\ a Université de Toulouse Jean Jaurès - ISTHIA, CERTOP - CNRS, France. \\ b Université Fédérale Toulouse Midi-Pyrénées, Université Toulouse Jean-Jaurès, LISST-CNRS, Toulouse, France.
}

Avec la méthode des focus groups, les enquêtés sont rassemblés par groupe, pour discuter d'un thème (le focus) selon un protocole bien défini (le script). Les discussions permettent la formulation de problèmes, l'identification de la diversité des opinions, des représentations et des pratiques, et leur mise en débat. Les phases de sélection des participants et d'analyse des discours recueillis sont essentielles à la qualité des données qualitatives ainsi collectées.

Le focus group est une méthode qualitative de recueil de données. Il s'agit d'une discussion collective semi-structurée entre participants sélectionnés, modérée par un animateur ou une animatrice en présence d'un assistant ou une assistante. Le focus group est considéré comme «une société pensante en miniature» (Farr et Tafoya, 1992, cités par Kitzinger et al., 2004 , p. 239), car cette méthode ouvre «une fenêtre sur la formation et l'évolution des représentations sociales, des croyances, des savoirs et des idéologies qui circulent sans les sociétés » (Marková, 2014 [2003], p. 223). Elle a pour but de collecter des données sur un nombre limité de questions relatives à un thème bien circonscrit (le focus), définies à l'avance par l'équipe de recherche. Ce temps d'échange est organisé autour de plusieurs phases et selon un guide d'animation (le script) défini en amont par les chercheurs.

Ce chapitre entend contribuer à la formalisation de la méthodologie des focus groups appliqués à l'alimentation. Cette contribution repose sur une revue de littérature et sur deux expériences de recherche conduites par les auteurs dans le domaine de l'alimentation. Questionnement d'autant plus intéressant du fait que les pratiques alimentaires sont banales, ordinaires, quotidiennes, routinières, familières, etc., et qu'elles sont difficiles, pour celui qui les met en œuvre, à remettre en question. Un des premiers avantages des focus groups est de favoriser la réflexivité sur de telles pratiques, ainsi que sur les représentations plus ou moins dissonantes avec les pratiques (Poulain, 2002). Mais, cette prise de parole peut être délicate lorsque l'objet de l'enquête est tabou ou controversé, ou encore lorsqu'elle vise un panel d'enfants. 
En aucun cas la contribution n'est exhaustive et la formalisation de cette méthode n'est applicable, telle une recette, à tous les objets de recherche. Elle demande d'être «bricolée». Nous présentons ainsi des «ficelles» issues de nos propres adaptations face aux difficultés rencontrées durant nos enquêtes (encadré 15.1). Le chapitre se concentre avant tout sur la mise en place de l'enquête et la collecte des données.

\section{Encadré 15.1. Usages de focus groups dans deux enquêtes}

La première enquête (Barrey et al., 2011) visait à saisir les préoccupations des mangeurs-consommateurs-citoyens ${ }^{*}$ vis-à-vis du premier animal génétiquement modifié destiné à la consommation humaine : le poisson transgénique. La particularité de l'enquête, en 2007-2009, résidait dans la non-connaissance, par les participants, de ce nouveau produit. Le focus group a permis de représenter l'objet méconnu sous différents formats (photos, articles de presse, traduction des différentes applications et de leurs objectifs). Il fait donc advenir les poissons génétiquement modifiés (POGM) comme un produit potentiel et a rendu possibles les réactions des participants, qui pouvaient alors énoncer, sous une forme complexe et argumentée, leurs appréhensions de ces biotechnologies et de la situation potentielle où les POGM seraient commercialisés.

La seconde enquête (Tibère et al., 2018) examinait le statut des deux prises alimentaires singulières, la collation du matin à 10 heures et le goûter de l'aprèsmidi à 16 heures, chez les enfants âgés de 3 à 15 ans. Les focus groups auprès des enfants et des adolescents ont été utilisés comme une méthode complémentaire aux entretiens individuels semi-directifs menés avec des parents. L'objectif était de donner la parole à ces jeunes mangeurs afin d'approfondir la compréhension du statut de ces deux repas et en particulier celui du goûter. Les focus permettaient d'accéder aux perceptions, aux imaginaires et aux habitudes de cette prise considérée comme un "petit repas». En effet, ce temps est moins étudié dans une perspective sociologique, alors qu'il est socialement régulé et encadré par des normes - temporelle, spatiale, en termes de contenu, etc. -, mais il présente des spécificités, notamment sur les manières de manger et les aliments consommés.

*Nous avons fait le choix de cette désignation pour marquer la manière dont les individus enquêtés ont été considérés : à la fois sous l'angle de leur rapport à l'alimentation, qui implique leur corps qui est aussi socioculturellement construit; sous l'angle de leur inscription dans les échanges socioéconomiques; sous l'angle, enfin, de leur participation sociopolitique au monde commun et à la vie de la cité.

\section{" Les premières mises en pratique des focus groups}

Les premières expériences de focus groups prennent place aux États-Unis durant la Seconde Guerre mondiale. Le contexte sociopolitique et scientifique, dans lequel le focus group a été conçu l'a prédestiné à un usage pour répondre à des questions importantes et urgentes émergeant dans le domaine de la communication de masse et de l'analyse de la propagande. Avec Paul Lazarsfeld, le sociologue américain Robert King Merton est le créateur de la méthode, qui ne s'appelle alors que «l'interview focussée» (Merton et Kendall, 1946). Très vite, elle est mobilisée par le marketing, en association avec des instituts de recherche académique qui l'utilisent dans des contrats de recherche avec des firmes privées. Le marketing était 
alors complètement intégré, par les études de marché, au monde de la recherche académique. Elle s'inspirait des techniques de dynamique de groupe utilisées par Carl Rogers, psychologue américain. Sous l'emprise des recherches expérimentales s'inscrivant dans le modèle behavioriste, cette méthode a presque totalement disparu pendant plus de quinze ans (Kitzinger et al., 2014). Dans les années 1980, l'outil réapparaît massivement dans le contexte des études de marché centrées sur les attitudes et les motivations des consommateurs, notamment dans le secteur de l'alimentation. Toutefois, depuis le milieu des années 1980, et encore davantage dans les années 2000, la technique jouit d'une popularité croissante dans les recherches qualitatives en sciences sociales, dont les revues publient plus de 100 articles par an utilisant cette méthode (Morgan, 1998). Les focus groups réintègrent ainsi leur contexte disciplinaire d'origine, la sociologie.

La sociologie de l'alimentation est particulièrement « friande » de l'outil, notamment lorsqu'elle adopte une approche par les représentations sociales et les valeurs dont l'étude de l'alimentation contribue à démontrer les effets. Cette méthode est appropriée au recueil de données lorsque l'on s'intéresse aux représentations sociales, car les focus groups sont fondés sur la communication et que celle-ci est au cour de la théorie des représentations sociales. En outre, parce que l'alimentation est soumise à des contraintes biologiques et écologiques, les sociologues de ce champ ont souvent travaillé en interdisciplinarité avec des sciences rompues à l'usage des focus groups (nutrition, médecine, etc.), ce qui a sans doute participé à la circulation de la méthode dans ce champ.

\section{Objectifs de connaissance}

En complément d'autres méthodes qualitatives, les focus groups permettent de répondre à cinq objectifs de connaissance :

- le problème social tel qu'il est formulé par les participants, et non tel qu'il est posé par les experts ou autres personnes détenant «la parole légitime»;

- les opinions des participants sur un sujet donné, dans le but de recueillir la plus grande hétérogénéité possible de points de vue (voire de pratiques sous certaines conditions) sur ce sujet;

- le type de connaissances des participants sur ce sujet. Il ne s'agit pas ici d'évaluer ces connaissances, ni de mesurer l'écart entre ce qui serait faux et ce qui serait vrai, mais plutôt de repérer si les personnes formulent leurs arguments en s'appuyant sur des données scientifiques, des données d'expérience, voire encore des croyances. Lorsque les participants échangent sur leurs points de vue, ils débattent aussi des sources et du degré de légitimité de ces connaissances. Ainsi, les focus groups ont aussi comme objectif de compréhension la fabrique des représentations et des prises de position;

- les représentations et les points de vue associés à des objets ou des thèmes non encore actualisés. Par exemple, avec l'enquête sur les poissons transgéniques, leurs représentations étaient un objet difficile à saisir du simple fait qu'ils ne sont pas présents aujourd'hui sur les étalages de vente en France et en Europe. Si nous partions de l'hypothèse que les consommateurs ne sont pas ces êtres dépourvus de rationalité souvent dépeints dans la littérature scientifique, la difficulté restait 
toutefois de les amener à construire un jugement ou une réflexion sur un objet quasi inexistant dans leur esprit. Aussi, nous avons choisi cette méthode pour faire émerger, sous une forme complexe et argumentée, les appréhensions vis-à-vis des poissons pêchés, élevés et génétiquement modifiés;

- appréhender le degré de consensus sur un sujet donné en faisant ressortir les points d'accord et de désaccord.

Le premier avantage de l'utilisation de la méthode présentée est l'abondance des données obtenues en peu de temps. Toutefois, le chercheur ne doit pas croire que sa réalisation avec 12 personnes remplacera 12 entretiens compréhensifs ${ }^{74}$ réalisés en face-à-face avec l'enquêté. Le choix d'une méthode n'a de pertinence qu'au regard des questions que l'on se pose, et les objectifs de connaissance du focus group ne recoupent que très partiellement ceux de l'entretien compréhensif. Le second avantage de cette méthode est la synergie créée par la présence du groupe de participants : écouter les autres parler peut stimuler la parole des participants selon ce que l'on appelle «l'effet boule de neige». Le dispositif les pousse à expliciter au maximum leur vision du sujet, leurs opinions et leurs comportements. Enfin, le focus group est une procédure souple qui donne une liberté de parole aux enquêtés, dans la mesure où ils discutent entre eux et sont donc moins soumis à la relation enquêteur-enquêté, davantage de mise dans la passation de questionnaires ou d'un entretien compréhensif. Les règles canoniques (Merton et Kendall, 1946) de la procédure insistent sur le fait que les organisateurs définissent avec précision les sujets à discuter (le focus), tandis que la séance doit se dérouler avec une réelle volonté d'écouter les participants et d'apprendre d'eux, selon l'adage «it is your focus but it is their group» (Morgan, 1998). La parole de chacun étant ainsi valorisée, les participants sont aussi impliqués dans l'échange.

Le focus group étant une méthodologie qualitative, la représentativité des données obtenues ne peut pas être assurée. Par ailleurs, et comme c'est aussi le cas pour l'entretien compréhensif, les résultats peuvent facilement amener à des erreurs d'interprétation si l'analyse ne prend pas en compte le contexte d'énonciation. Ensuite, l'analyse des données est «lourde». À titre d'exemple, un focus group de 3 heures produit entre 60 et 80 pages de corpus à analyser. Enfin, et ce n'est pas vraiment une limite lorsque le chercheur en est conscient, le focus group n'est pas «neutre», les participants en sortent «transformés» (nouvelles connaissances, nouveaux points de vue, etc.). Peut-être encore plus que l'entretien individuel, le partage de nouveaux points de vue encourage fortement la réflexivité des personnes.

Parler de l'alimentation, tout comme d'autres sujets (la sexualité, le harcèlement, etc.), peut faire partie des tabous, porter sur l'intime, ou encore soulever des débats passionnés. Cette méthode ne constitue alors pas toujours la meilleure méthode pour encourager la parole. Il ne faut pas cependant en conclure que la présence du groupe inhibe davantage les participants que lors d'un entretien en face-à-face. C'est parfois tout le contraire qui opère : la situation de groupe peut faciliter une discussion du fait que certains individus «moteurs de parole» entraînent les plus inhibés dans une synergie. L'échange peut également fournir un soutien mutuel,

74. Les entretiens compréhensifs ont pour finalité de recueillir des faits auprès des enquêtés. Ils ont pour but de saisir leur point de vue, autrement dit la manière dont ils interprètent et donnent du sens au monde, à leurs pratiques et à leur expérience. 
en ce qu'il permet l'expression d'idées ou de pratiques s'écartant de la norme (ou supposée telle chez le chercheur) et les silences feront partie intégrante de l'analyse. L'animateur a un rôle important à jouer pour pallier certaines de ces limites. Il doit contrôler les effets de groupe, nuisibles à l'émergence de nouveaux arguments et à la prise de parole. Plusieurs situations peuvent se présenter et seront exposées ci-après.

Enfin, des limites plus sévères quant à l'usage de cette méthode ont par ailleurs été avancées dans les années 1980 par ses fondateurs. Merton (1987) en critiquait un emploi impropre, lorsqu'elle était intensivement utilisée pour des études de marché et que des manuels de marketing utilisaient le terme de focus group comme une technique particulièrement pertinente pour les étudiants se formant aux études de marché. Merton considérait la méthode utile au marketing, bien sûr, mais pas seulement pour la recherche marketing. Selon lui, il s'agissait plutôt «d'un ensemble de procédures pour la collecte et l'analyse de données qualitatives qui peuvent nous aider à acquérir une compréhension sociologique et psychologique élargie dans n'importe quelle sphère de l'expérience humaine» (Merton et Kendall, 1946, p. 565). La méthode est encore critiquée aujourd'hui en France à ce titre lorsqu'elle est mobilisée à d'autres finalités que la compréhension sociologique (Touré, 2010).

\section{" Enjeux éthiques et juridiques}

Les risques associés aux traitements sur les droits et libertés des personnes, aux menaces possibles et aux mesures existantes ou prévues pour y répondre sont liés aux spécificités de l'objet étudié et de la population interrogée.

Durant les focus groups, la production de données collectives qui est très souvent filmée, cristallise la dimension réglementaire de la protection des données. D'autant plus que l'élargissement de l'environnement numérique et la diffusion large et rapide des informations ont fait évoluer les textes réglementaires. Dernièrement, le règlement général sur la protection des données (RGPD) est entré en application le 25 mai 2018 pour organiser la protection des données à caractère personnel au niveau européen. Les programmes de recherches en sciences humaines et sociales intégrant des données à caractère personnel - données d'identification (prénom, lieu d'habitation, voix, visage, etc.), données d'identification indirecte (par exemple, le croisement d'informations : secteur d'activité et lieu de travail), mais aussi données dites «sensibles » (données de santé, origine ethnique, appartenance syndicale, etc.) - nécessitent une protection des informations des personnes impliquées dans les recherches. Cette réglementation encadre les pratiques des chercheurs dans l'ensemble du processus de production des connaissances, c'està-dire lors de la collecte des données, de leurs traitements, stockages et diffusions. Cette avancée pour les enquêtés entraîne, néanmoins, des contraintes dans la mise en œuvre des mesures techniques et organisationnelles de l'enquête. Pour garantir la confidentialité et la «sécurité » des données collectées et éviter leur divulgation, le consentement des participants est requis. Ce dernier, souvent validé sur le principe en amont de la réalisation du focus group, est consigné le jour de sa réalisation.

Avec cette méthode, les participants partagent des informations plus ou moins sensibles et/ou confidentielles avec l'animateur, mais également avec les autres 
enquêtés. Ainsi, il est difficile de maintenir un niveau de confidentialité élevé. Lorsque, nous travaillons avec des groupes déjà formés (professionnels d'une même entreprise, par exemple), l'information obtenue pendant une session peut faire l'objet d'un commérage local, voire d'une publicité et, dans certains cas, peut être source de conflit et de stress. De plus, les informations issues des connaissances scientifiques formulées par les participants peuvent être inexactes ou erronées (c'était le cas, par exemple, sur les OGM). L'animateur se doit donc, au début du focus, de rappeler les consignes de confidentialité (non-divulgation des discussions du groupe par les membres participants, et protection des informations collectées par le chercheur) et, à la fin du focus, de rectifier et d'apporter des connaissances scientifiques stabilisées, quand c'est possible.

\section{W Un outil d'exploration du social utile à d'autres types d'enquêtes}

Si la méthodologie des focus groups ne vise pas la représentativité des résultats, les données constituées par le dispositif méthodologique peuvent contribuer à dégager certains cas (des histoires de vie, des argumentations, des pratiques et des représentations, etc.) comme exemplaires de phénomènes plus généraux. En effet, la multiplication des focus groups et l'assurance d'enquêter ainsi auprès d'individus aux profils très variés permettent de rendre compte, dans l'analyse, de situations de vie et d'argumentations hétérogènes. Cette interprétation des données ne vise pas la recherche d'un «tout» ou d'un «fait alimentaire total» qu'il aurait fallu découvrir ou révéler (comme dans une approche déterministe révélant des facteurs explicatifs des comportements et des représentations), mais comme une collecte de ressources très diverses qui ne peuvent être rattachées à une culture commune ou à un espace social commun aux participants des focus groups. Toutefois, l'usage de ces cas hétérogènes permet des montées en généralité, dans le sens de ce que Nicolas Dodier et Isabelle Baszanger (1997) ont appelé une «ethnographie combinatoire, qui, en circulant simultanément sur différents terrains, réunit une jurisprudence de cas propre à identifier les différentes formes d'action dans lesquelles les personnes peuvent s'engager, ainsi que leurs combinaisons possibles» (p. 38). L'enquêteur ne se fixe donc pas sur un groupe déjà constitué «à partir duquel il reconstituerait une entité collective. Il circule entre plusieurs chantiers, au fur et à mesure des dimensions qui apparaissent pertinentes dans l'analyse de chaque cas» (p. 51.). Chaque nouveau focus group constitué est réalisé en vue d'atteindre un effet de saturation, parce que l'analyste s'attend à y trouver une forme d'action ou un argumentaire inexistant jusque-là et qui le force à le prendre en compte. La généralisation ainsi produite à partir de la multiplication des cas ne vise pas tant à la totalisation des données sur des entités d'appartenance sociale ou culturelle, qu'à la mise en évidence d'une combinatoire entre des formes d'action et d'argumentation hétérogènes, voire contradictoires.

Cette totalisation combinatoire n'est possible que par la standardisation du protocole d'enquête qualitatif, qui permet sa reproductibilité via un guide d'animation commun aux différents focus groups. Enfin, comme tout autre outil de recueil de données, les focus groups peuvent être le point de départ (en tant que méthodologie exploratoire) à d'autres méthodes de recherche (qualitatives ou quantitatives) 
visant à appréhender les phénomènes sociaux sous un autre angle. L'utilisation exploratoire des focus groups peut nourrir des recherches en sciences humaines et sociales aussi bien qu'en nutrition, par exemple pour préparer des enquêtes sur la consommation alimentaire en les adaptant au mieux à leur contexte.

\section{- Mise en pratique illustrée par deux cas d'application}

L'élaboration et la mise en place des focus groups se font en plusieurs étapes méthodologiques. Dans le cadre limité d'un chapitre, il est difficile de présenter en détail l'ensemble des étapes. Il s'agit davantage, dans cette partie, de rendre compte de celles qui nécessitent des ajustements pratiques (figure 15.1) ${ }^{75}$.

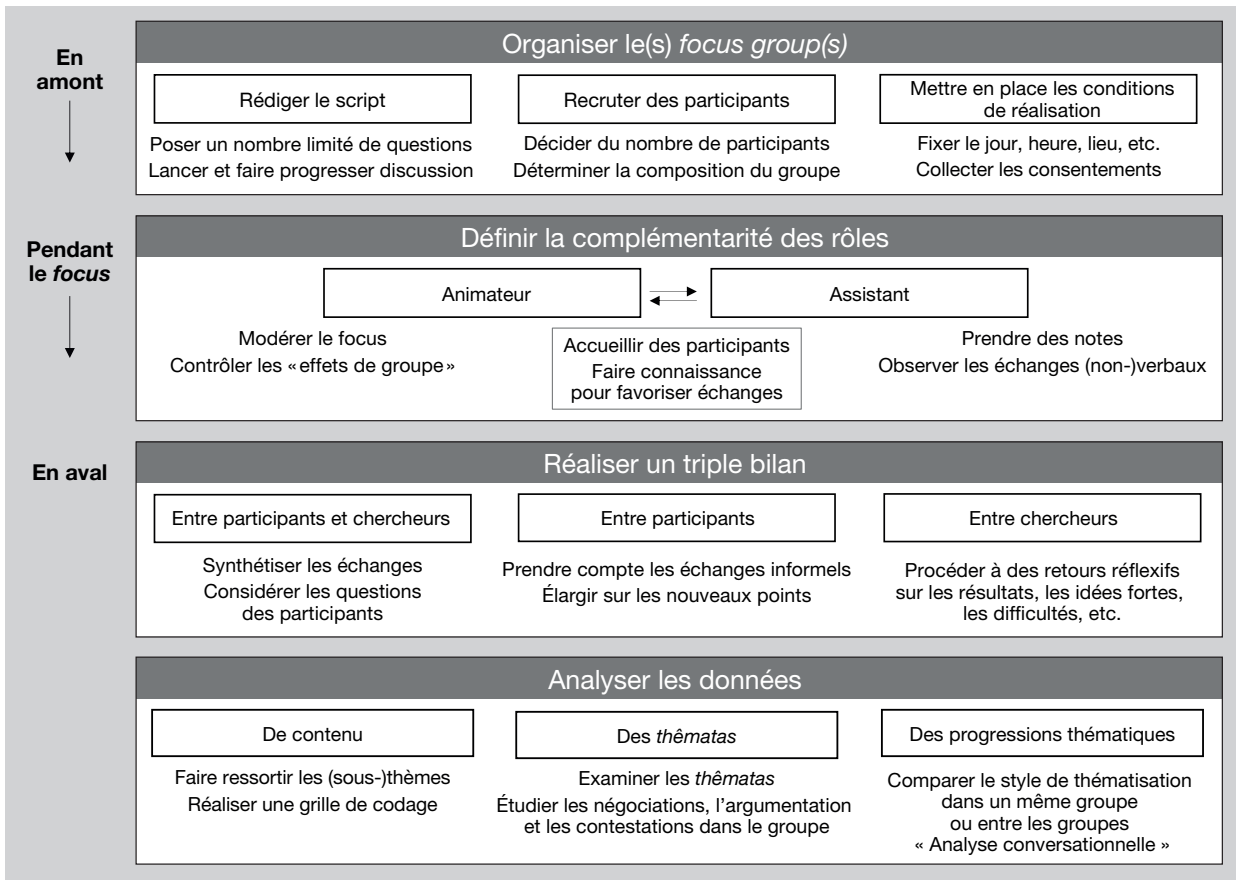

Figure 15.1. Les étapes de la démarche du focus group.

\section{Organiser le(s) focus group(s)}

Il s'agit tout d'abord d'élaborer le guide d'animation : le script. Ce dernier doit répondre à plusieurs objectifs : favoriser l'émergence de la plus grande hétérogénéité possible d'opinions, d'arguments et de justifications; susciter l'explicitation des propos tenus par chacun des participants; et encourager l'énonciation de récits et d'anecdotes. Le déroulement précis de la séquence favorise, d'une part, la reproductibilité entre chaque focus group et, d'autre part, l'équilibre entre les temporalités des thèmes du focus et

75. Ainsi, nous nous concentrons davantage sur le recueil des données que sur leur analyse. Sur cet aspect, nous renvoyons les lecteurs à Markova (2014 [2003]). 
celles de l'expression de tous les participants. Il se découpe généralement en plusieurs parties. Tout d'abord, la consigne de départ se veut à la fois précise et générale afin de définir clairement le(s) thème(s) de la discussion et de ne pas enfermer les discours dans un cheminement trop réducteur. Elle doit également interpeller d'emblée les participants afin qu'ils se sentent immédiatement impliqués dans la discussion. Ensuite, à travers un nombre limité de questions, le guide permet de lancer et de faire progresser la discussion du groupe autour des thèmes souhaités. L'utilisation de photographies, d'objets ou de documents peut être associée au script afin de faciliter la narration d'événements concrets par les participants. Par exemple, dans l'enquête sur les POGM sur les marchés français, le guide d'animation était incrémenté de photographies ${ }^{76}$ faisant référence à des situations concrètes d'achat (dans la première partie); à différents types d'élevage (dans la deuxième partie) et à différentes applications alors en cours sur les POGM (dans la troisième partie). Les participants ont pu ainsi respectivement faire référence à leurs propres manières de choisir et de s'approvisionner en poisson, et faire valoir leurs opinions quant aux différents cas présentés.

Ensuite, le «recrutement» des participants - qu'ils soient profanes ou experts - doit permettre la représentativité des différents acteurs impliqués par l'objet de recherche. Le groupe se compose d'environ 6 à 15 personnes et «la sélection» demande un équilibre. Un groupe «hétérogène», en termes de profils socio-économiques ou de partage de contexte d'action, peut amoindrir la spontanéité des échanges ou susciter de la retenue de la part de certains participants à faire part de leurs expériences personnelles. À l'inverse, un groupe «homogène» peut entraîner des échanges peu contrastés ou sans reliefs alors que la technique vise à explorer différents points de vue par la discussion. Dans le cadre de l'enquête sur le goûter, la présence d'enfants et d'adolescents en 2 groupes d'âge - les 7 à 10 ans et les 11 à 15 ans - a permis de distinguer l'univers des plus jeunes de celui des adolescents. De plus, la scission par âge a mis en exergue les différences (dans les normes et dans les pratiques) socialement situées en fonction du genre, des catégories socioprofessionnelles des parents, de la présence d'une fratrie et des lieux de prise (domicile ou hors domicile).

Enfin, la mise en place de focus group peut être difficile à organiser et à mettre en place du fait de la nécessité de regrouper plusieurs individus dans le même lieu et au même moment. Après l'accord de l'enquêté (et de ses représentants légaux pour les enfants), et afin de limiter le nombre d'absents, il est important de notifier : le jour, l'heure, la durée et le lieu du focus group ${ }^{77}$. De plus, des informations peuvent être

\footnotetext{
76. Dans le cadre de cette recherche, une question se pose toutefois sur la mobilisation de photos ou d'informations «réelles» ou «truquées». Lorsque nous avons commencé l'élaboration du guide d'animation pour l'étude sur les POGM, un collègue avait soumis l'idée de montrer une photo d'une boîte de thon mentionnant une fausse allégation du type «sans OGM» (cette allégation est interdite en Europe). Après réflexion, il avait été décidé de ne mobiliser que des photos ou documents réels afin de ne pas ajouter d'incertitudes à un sujet déjà extrêmement complexe.

77. Pour stimuler la discussion collective, le lieu se veut neutre, agréable, accessible à la population étudiée et adapté (table, matériel de projection, etc.). Aujourd'hui, il est possible de réaliser des focus groups dans une salle dédiée (notamment au sein des instituts de sondage ou dans les universités), comme cela a été le cas pour l'étude sur le goûter des enfants et des adolescents. La salle située en centre-ville de Toulouse était facile d'accès pour les familles. Elle a été aménagée à la convenance du chercheur et mettait à disposition un équipement technique (enregistrement, diffusion d'image, etc.). De plus, la salle était équipée d'une glace sans tain et a permis à l'assistante de suivre le focus derrière en direct et procéder à la pré-analyse. Dans ce cas, les échanges avec l'animateur ont été réalisés lors de la pause.
} 
données sur le contexte dans lequel le focus est organisé; le(s) sujet(s) abordé(s) et présenté(s) de manière attractive; ainsi que l'utilisation qui sera faite, des informations obtenues et les assurances en matière de confidentialité permettent de réassurer les participants. Il est indispensable de demander l'accord préalable à l'ensemble des participants ${ }^{78}$ pour l'enregistrement audio ou vidéo. L'enregistrement audio assure la traçabilité des données et permet l'obtention de données de meilleure qualité qu'une simple prise de notes. Cependant, l'enregistrement vidéo est privilégié pour deux raisons : d'une part, il est utile au moment de la retranscription pour visualiser qui prend la parole; d'autre part, il permet d'enregistrer et d'analyser les réactions mimiques ou gestuelles des participants. Ces réactions apportent des informations au même titre que les prises de parole et ne sont pas toujours faciles à noter lors de la réalisation du focus group (même si l'observateur s'intéresse à ces aspects).

\section{Définir la complémentarité des rôles}

L'utilisation de cet outil méthodologique implique généralement deux personnes dont les rôles sont complémentaires : un animateur et un assistant.

L'animateur, aidé de l'assistant, doit amener les enquêtés à faire connaissance, soit en leur laissant le temps de discuter entre eux, soit en suscitant une petite discussion collective pour se présenter ou donner les raisons qui les ont amenés à participer. L'intérêt de cette étape préalable est de créer une ambiance favorable et de lever toute inhibition afin que, lorsque le focus group commence, les individus soient suffisamment à l'aise pour prendre librement la parole. Par ailleurs, cet échange informel autour des raisons qui les ont poussés à participer constitue souvent une «mine d'informations» pour la recherche. Avant que la discussion commence, il est souhaitable de rappeler le contexte de la recherche, les objectifs et d'énoncer les «règles du jeu» afin que tout le monde puisse s'exprimer dans les meilleures conditions. L'une de ces règles est de ne pas prendre la parole en même temps. Elle a un intérêt double : elle permet l'écoute mutuelle et facilite le travail de celui qui retranscrira intégralement la discussion. Par la suite, l'objectif principal de l'animateur est de faire progresser la discussion selon un canevas très précis, décrit par le script. Par exemple, dans le cadre de la recherche sur le goûter des enfants, nous avons essayé d'affranchir les enquêtés de leurs «normes personnelles» liées aux traditions culturelles, sociales et familiales en les questionnant dans un premier temps sur ce qu'ils considèrent comme un «vrai goûter». Dans un second temps, nous avons reconstruit la pluralité des pratiques selon les contextes physiques (au domicile ou à l'extérieur du domicile; chambre, cuisine ou salon) et selon les contextes sociaux (pairs, fratrie, parents, grands-parents, etc.). Nous avons terminé sur les questions des représentations du goûter en lien avec la santé.

Une des difficultés majeures pour l'animateur est de contrôler les «effets de groupe » qui peuvent être nuisibles à l'émergence de nouveaux arguments. Plusieurs situations peuvent se présenter :

- un participant ne prend jamais la parole et reste dans l'écoute de ce que disent les autres. Quelle que soit la raison (timidité, faible intérêt pour le sujet, etc.),

78. Une demande spéciale pour les mineurs est requise. 
l'animateur se doit d'impliquer cette personne en s'adressant directement à elle lors d'une nouvelle question ou d'un nouveau thème («Et vous, qu'est-ce que vous en pensez?»);

- d'autres participants, à l'inverse, mobilisent le temps de parole en donnant systématiquement leur avis en premier, en voulant «avoir le dernier mot» ou encore en contestant systématiquement la parole des autres. Lanimateur est tenu de limiter ce type de pratiques avant que les autres participants n'osent plus s'exprimer, ou aillent toujours dans le même sens que le ou les leaders. Pour cela, il a la possibilité de s'appuyer sur ses propos en facilitant la prise de parole des autres ( C'est un point de vue intéressant. Les autres, vous en pensez quoi ?»);

- un participant a des difficultés à rester sur le sujet et dévie la question initialement posée. Lanimateur peut ramener vers le sujet comme précédemment en soulignant l'importance de l'avis de chacun;

- des alliances se tissent entre des personnes présentes dans le focus et la discussion se polarise entre les «camps». Dans ce cas, l'animateur est autorisé à suggérer un autre point de vue (par exemple, un point de vue entendu dans un autre focus group) afin d'élargir la discussion;

- certains participants ont tendance à suivre systématiquement l'opinion majoritaire, tel l'effet de «désirabilité» bien connu en méthodes qualitatives. Pour atténuer cela, l'animateur peut conduire ce ou cette participant(e) à davantage donner son point de vue.

Ainsi, l'animateur a pour fonction de garder le contact visuel avec l'ensemble des participants, de faire approfondir et de préciser les propos ( «pouvez-vous donner un exemple concret?»), de reformuler les propos, de demander confirmation pour être certain d'avoir bien compris et enfin de demander d'autres points de vue («est-ce que quelqu'un voit cela différemment?»). Son statut lui demande également de rester neutre tout au long de l'animation des débats (ne pas prendre parti pour un participant, mais valoriser au contraire la parole de tous), d'être décontracté et de ne pas apparaître trop «professionnel» ou «inhibant», afin de provoquer une discussion constructive, sans toutefois nuire au sérieux du sujet, et de faire la synthèse des discussions.

Dans l'idéal, la mise en place de cette méthodologie requiert l'aide d'un assistant. Pour ce dernier, son implication se trouve dès l'amont du focus group (mise en place des enregistrements, accueil des personnes, récupération des autorisations d'enregistrement, etc.). À titre d'illustration, lors de l'enquête avec les enfants, la présence simultanée de l'animatrice et de l'assistante permettait de pouvoir accueillir l'enfant, le(s) parent(s) et de gérer les modalités pratiques (consentement, heure de fin du focus group, gestion des allergies, etc., car un goûter permettait de couper le temps du focus en deux). L'accueil des participants permet d'instaurer une ambiance chaleureuse. Pendant le focus, la gestion des aspects techniques, par exemple le lancement de l'enregistrement ou la vérification de l'enregistrement, nécessite la présence de cette seconde personne. L'assistant s'assoit généralement en retrait du cercle, mais cet endroit doit lui permettre de voir l'ensemble des participants ainsi que l'animateur, afin de faciliter les échanges. En se positionnant vers la porte ou derrière la vitre sans tain, il peut également accueillir les éventuels retardataires et leur expliquer les consignes en dehors de la pièce sans interrompre le focus. Néanmoins, le 
rôle principal de l'assistant est de prendre des notes, d'observer les échanges et surtout d'apporter son soutien dans la maitrise du groupe et dans la progression de la discussion. Ces notes permettront de reprendre les «phrases clés» avec ou sans les commentaires personnels, l'ordre des interventions, les attitudes et expressions non verbales des participants. En effet, avec un regard plus décentré, l'assistant peut repérer certains éléments dans le déroulement du focus et dans l'analyse.

\section{Réaliser un triple bilan}

À la fin du focus, l'animateur avec l'assistant, réalise une synthèse finale. Elle permet de demander aux participants s'ils sont en accord avec la synthèse et si rien n'a été oublié. Ce moment permet également de laisser de la place aux questions des participants. Comme à la fin d'autres méthodes de recueil de données, «on ne se quitte pas quitte!» : outre les classiques remerciements et petits dédommagements adressés à la fin de la discussion, il est souhaitable d'informer les participants du fait que les résultats de l'étude leur seront communiqués. Cette restitution peut d'ailleurs faire l'objet d'un nouvel entretien groupé pour éprouver les pistes d'analyses retenues et les principaux résultats.

En outre, des liens et/ou parfois des tensions ont pu se créer entre les participants lors de la discussion. Par conséquent, il est intéressant de réserver un temps en fin de session pour les laisser discuter entre eux et les écouter. Cette étape peut se dérouler «en off», sans enregistrement et de manière plus informelle. Ce moment est ainsi propice à des prolongements de discussion ou à des ouvertures sur de nouveaux points d'échange. De plus, des participants peuvent avoir des questions à poser (car ils n'ont pas osé pendant le focus group ou qu'il était impossible de répondre à ce moment-là) à l'animateur ou à un autre individu présent.

Enfin, un débriefing entre l'animateur et l'assistant est essentiel à la fin de chacune des séances. Ce retour réflexif permet de noter les réactions «à chaud» comme les difficultés rencontrées, les idées fortes du groupe ou encore les résultats non anticipés.

\section{Analyser les données}

Brièvement, nous aimerions évoquer quelques pistes pour l'analyse des données issues des focus groups, même si, comme le souligne Mavorká (2014 [2003]), celleci n'est pas encore très élaborée et diffusée dans les manuels consacrés à cette méthode. Marková repère quatre types d'analyse pouvant s'opérer séparément ou en se complétant mutuellement :

- l'analyse de contenu, consistant à construire les thèmes et sous-thèmes liés aux paroles des enquêtés. Ce type d'analyse est précieux pour dégrossir dans un premier temps le corpus très conséquent des données verbales;

- l'analyse des thêmatas, dans la tradition théorique de Moscovici (1994). Il s'agit des unités linguistiques propres à une génération ou à une culture, transmise par le langage, du type «bon/mauvais», «dominant/dominé», «juste/injuste», etc. Pour étudier le fait alimentaire, cette analyse nous semble particulièrement pertinente en elle-même, mais aussi parce qu'elle est potentiellement utile pour analyser l'évolution de ces signifiants sur le long terme. Ainsi, sur les aliments OGM, l'analyse des 
thêmatas permet aux analystes de repérer d'une part des catégorisations telles que «vivant/non vivant», «comestible/non comestible», «aliment/produit», et d'autre part des remises en question, avec le temps, de ce qui semblait aller de soi. Par exemple, nos premiers focus groups en 2006-2007 n'ont pas fait émerger l'opposition entre «bien-être» et «souffrance» des animaux. Pourtant deux ans après (de nombreux reportages avaient été diffusés sur l'élevage du saumon), les poissons se retrouvaient, dans la parole des enquêtés, eux aussi inscrits dans la catégorie des animaux sensibles (avec les mammifères d'élevage et les animaux domestiques), ou subissant un «stress» ou encore une «souffrance». Dans l'étude de l'alimentation, ce qui semble aller de soi peut donc se retrouver problématisé ou remis en question; - l'analyse de la progression thématique est pertinente lorsque l'animateur du focus group apporte des connaissances aux participants (ce qui est le cas lorsque l'objet de la discussion collective est assez méconnu). Le chercheur peut étudier l'évolution des argumentations en rapport avec la «progression» des connaissances des enquêtés;

- l'analyse conversationnelle est dans la lignée du point précédent, mais elle s'opère à une échelle plus microsociologique, puisqu'elle vise à prendre en compte le contexte d'énonciation du focus group. Elle illustre notamment l'enracinement communicationnel du contenu énoncé, et la façon dont le déroulement des interactions participe aussi à la production de ces énoncés. L'analyse se centre sur «ce qui est dit» et sur «la manière dont cela est dit».

Enfin, il nous semble également pertinent de mobiliser, en complément de ces types d'analyse, une étude biographique et personnelle des participants. Les corpus des focus groups regorgent de ce type de données, nécessaires à contextualiser la parole des enquêtés, leurs ressources et leur trajectoire.

\section{" Une méthode adaptable aux objets, à la population et plus largement aux contextes}

Lorsque l'objet abordé en focus group fait l'objet de vives controverses, ou est susceptible d'être débattu entre des participants hétérogènes, il est souhaitable d'adapter la succession des thèmes abordés dans l'échange. Comme ce fut le cas lors de notre recherche sur les POGM, il est bienvenu de structurer le script de façon à aborder les points les plus sensibles à la fin de l'échange, et de privilégier des questions ouvertes et moins engageantes au début. Ainsi, sur des sujets alimentaires tels l'équilibre alimentaire, les OGM, les alicaments ou les relations aux plats très transformés, la première partie du script peut être consacrée aux habitudes alimentaires (les goûts, les pratiques d'achat, les lieux d'approvisionnement, les choix, etc.); les questions d'opinion étant davantage abordées dans une autre phase de l'échange, une fois les participants mis en confiance.

Quant au focus avec les enfants, cette méthode a permis de favoriser «la diversité et la divergence d'opinion[s], ainsi que le[ur] consensus» (Mavorka, 2014 [2003], p. 221) des normes du goûter. La méthodologie a créé de la synergie entre les enfants ou adolescents et a limité, voire inversé l'asymétrie enquêteur/enquêté que l'on retrouve dans l'entretien individuel. De plus, dans le cadre de l'enquête sur les petits repas, l'utilisation de cette méthode a permis de recueillir des informations auprès des 
mangeurs concernés par le goûter en compléments de discours parentaux et d'éviter la pression de l'entourage familial et le conformisme parental auquel nous aurions dû faire face si nous avions réalisé des entretiens individuels avec les enfants, au domicile et en présence de leurs parents. Lorsque nous avons réalisé les entretiens semi-directifs auprès des parents, leurs enfants pouvaient faire une apparition et être invités par leurs parents et/ou la chercheuse à faire part de leur expérience. Les propos de l'enfant étaient d'une grande richesse, par exemple sur le goûter à l'école (normes et pratiques), mais ils pouvaient également perturber l'entretien individuel avec les parents. Deux exemples éclairent cette situation délicate. Premièrement, dans une famille, les propos d'un enfant ont été censurés par sa mère et elle lui a demandé de quitter la salle à manger, car il a donné des exemples contraires à ce qu'elle démontrait. Elle valorisait les «bons goûters» d'un point vu nutritionnel alors que l'enfant invoquait des exemples de «mauvais goûters», nutritionnellement parlant, au domicile en déconstruisant la norme de la «bonne parentalité» de sa mère. Une fois partie, la mère a souligné que l'enfant «voulait attirer l'attention » pour reprendre ses propos. Deuxièmement, un adolescent a expliqué à demi-mot, quand sa mère est allée chercher de l'eau dans la cuisine, que les goûters étaient très différents selon les contextes, notamment chez son père lors de la garde alternée et avec ses amis. Mais, il ne pouvait pas en dire plus, car sa mère revenait de la cuisine. C'est ainsi qu'il nous a semblé pertinent d'utiliser les focus groups auprès de 19 enfants $^{79}$ (7 à 10 ans) et adolescents (11 à 15 ans), en complément des entretiens individuels avec des parents. Cependant, la réalisation de focus groups avec des enfants a demandé la mise en place d'adaptations. Il a été nécessaire d'apporter des outils ludiques et variés afin de maintenir une attention soutenue, ne pas polariser la discussion animateur-participant et stimuler la discussion collective. En plus des images pour décrire les aliments consommés, nous avons mobilisé des techniques projectives associées au dessin : "Si tu étais un goûter, à quoi tu ressemblerais?» (figure 15.2).
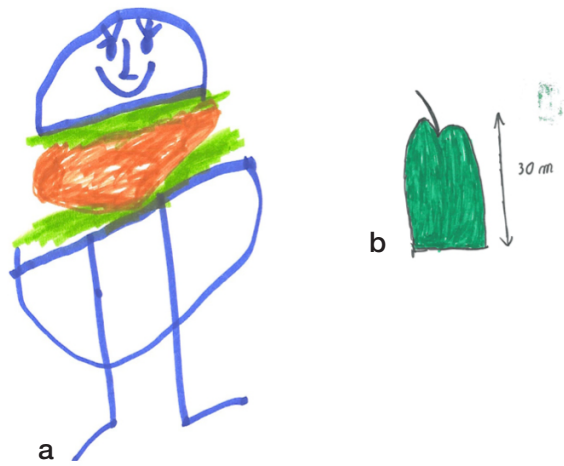

Figure 15.2. Une technique projective pour appréhender les représentations alimentaires ${ }^{80}$.

79. Les enfants des focus groups n'étaient pas les enfants des parents enquêtés lors de la première phase pour ne pas orienter leurs propos.

80. Le dessin de gauche (a) a été décrit par sa jeune dessinatrice comme «un sandwich avec des choses bonnes pour la santé et d'autres que j'aime bien» (Soledad, 7 ans), tandis que le jeune dessinateur de celui de droite (b) a déclaré que son dessin représentait «une pomme de 30 mètres pour que l'on me partage » (Thomas, 10 ans). 
Pour chaque enfant, la réalisation d'un dessin individuel a succédé à un exposé et une explicitation au reste du groupe. Au-delà de la dynamique argumentative, cette question a permis d'obtenir une réponse individuelle qui n'a pas été influencée par le reste du groupe et donc d'étudier là aussi les jeux de conversation.

La méthode desfocus groups est encore aujourd'hui peu formalisée, malgré son usage important en sciences sociales. Nous avons souligné ses singularités par rapport à d'autres techniques apparemment semblables, comme les focus groups en marketing, les groupes de parole en psychologie, ou encore d'autres formes d'échanges collectifs ${ }^{81}$, dont les visées opérationnelles guident, voire parfois déterminent très fortement le déroulement des échanges. S'il est possible d'opérationnaliser les résultats obtenus par les focus groups a posteriori, il nous semble périlleux d'utiliser cet outil sans la vigilance critique propre à la discipline sociologique.

\section{" Références}

Barrey S., Mariojouls C., Pucheu E., 2011. Lappréhension des POGM par les consommateurs : des modes d'existence de la nature et du marché, in Barrey S., Kessous E. (éd.), Consommer et protéger l'environnement. Opposition ou convergence? Paris, L'Harmattan, 181-202.

Dodier, Nicolas, Isabelle Baszanger. 1997. Totalisation et altérité dans l'enquête ethnographique. Revue française de sociologie, 38 (1), 37-66. https://doi.org/10.2307/3322372

Kitzinger J., Marková I., Kalampalikis N., 2004. Qu'est-ce que les focus groups? Bulletin de psychologie, 57/3 (471), 237-243. [En ligne] https://www.bulletindepsychologie.net/vente/Les\%20 groupes\%20centres\%20(focus\%20groups).pdf (dernière consultation le 31/08/2021).

Marková I., 2014 [2003]. Focus Groups, in Moscovici S., Buschini F. (éd.), Les méthodes des sciences humaines, Paris, Presses universitaires de France, 221-243.

Merton R. K., 1987. The Focussed Interview and Focus Group: Continuities and Discontinuities. Public Opinion Quarterly, 51 (4), 550-566.

Merton R. K., Kendall P. L., 1946. The focussed interview. American Journal of Sociology, 51 (6), 541-557. https://doi.org/10.1086/219886

Morgan D. L., 1998. The Focus Group Guidebook, Thousand Oaks, Sage Publications, 103 p. http:// dx.doi.org/10.4135/9781483328164

Moscovici S., Vignaux G., 1994. Le concept de thêmata, in Guimelli C. (éd.), Structures et transformations des représentations sociales, Neuchâtel, Delachaux et Niestlé, 25-72.

Poulain J.-P., 2002. Sociologies de l'alimentation, Paris, Presses universitaires de France, 286 p.

Tibère L., Rochedy A., Charlotte S., 2018. Le goûter résiste à la nutritionnalisation. Cahiers de nutrition et de diététique, 53 (4), 232-239. https://doi.org/10.1016/j.cnd.2018.03.008

Touré E. H., 2010. Réflexion épistémologique sur l'usage des focus groups : fondements scientifiques et problèmes de scientificité. Recherches qualitatives, 29 (1), 5-27. [En ligne] http://www. recherche-qualitative.qc.ca/documents/files/revue/edition_reguliere/numero29(1)/RQ_Toure.pdf (dernière consultation le 31/08/2021).

81. Telle l'intervention sociologique utilisée par Alain Touraine, qui visait à aider un collectif (d'antinucléaires par exemple) à formaliser leurs arguments pour mieux les porter et les faire entendre sur les scènes publiques et politiques. 


\section{Liste des auteurs}

Grégori Akermann est sociologue, chargé de recherche à l'INRAE. Spécialisé en sociologie économique et des réseaux, il s'intéresse aux consommations alimentaires émergentes dans un contexte de développement des circuits de proximité. Contact : gregori.akermann@inrae.fr

Carolyn Imelda Auma est maîtresse de conférences à la School of Food Science and Nutrition de l'université de Leeds. Elle s'intéresse aux transitions des comportements alimentaires et aux maladies non transmissibles liées à l'alimentation dans les pays à revenu faible ou intermédiaire, ainsi qu'aux pratiques alimentaires durables et favorables à la santé. Contact : C.I.Auma@leeds.ac.uk

Sandrine Barrey est maîtresse de conférences en sociologie à l'université de Toulouse-II JeanJaurès et membre du LISST-CNRS. Ses travaux portent sur les marchés controversés des biotechnologies alimentaires et plus généralement sur l'émergence de nouvelles relations au vivant. Contact : sandrine.barrey@univ-tlse2.fr

Anne Bichard est chargée de programme Sécurité alimentaire et nutritionnelle à l'Iram. Elle intervient dans le cadre de projets opérationnels ou de recherche. Elle s'intéresse particulièrement à l'évolution des pratiques de consommation alimentaire en Afrique subsaharienne. Contact : a.bichard@iram-fr.org

Alison Blay-Palmer est professeure de géographie et d'études environnementales à la Wilfrid Laurier University de Waterloo, au Canada, où elle est titulaire de la Chaire Unesco d'études sur l'alimentation, la biodiversité et la durabilité. Contact : ablaypalmer@wlu.ca

Douadia Bougherara est chargée de recherches à l'INRAE et travaille sur les écolabels, les programmes agro-environnementaux et l'aide à la décision pour la réduction des pesticides, sur le rôle des préférences pour le risque et des préférences sociales. Contact : douadia.bougherara@inrae.fr

Emmanuelle Bouquet est économiste au Cirad. Elle mène des recherches sur les conditions de vie des ménages ruraux dans les pays du Sud, en privilégiant un travail de terrain basé sur des méthodes mixtes et le dialogue entre disciplines. Contact : emmanuelle.bouquet@cirad.fr

Nicolas Bricas est chercheur en socio-économie de l'alimentation au Cirad (MoISA), titulaire de la Chaire Unesco Alimentations du monde et co-directeur du master spécialisé «Innovations et politiques pour une alimentation durable (MS IPAD)». Contact : nicolas.bricas@cirad.fr

Michaël Bruckert est docteur en géographie et chercheur au Cirad (UMR Innovation). Ses recherches portent sur l'alimentation carnée et sur les systèmes alimentaires urbains en Asie. Il est l'auteur de La chair, les hommes et les dieux. La viande en Inde aux Éditions du CNRS (2018). Contact : michael.bruckert@cirad.fr

Paul Cœurquetin est doctorant en sociologie. Ses recherches portent sur l'émergence de nouveaux rapports aux micro-organismes portés par de nouvelles connaissances, promesses, pratiques et prescriptions, dans les sphères scientifique, institutionnelle, marchande et domestique. Contact : paul.coeurquetin@gmail.com

Géraldine Comoretto est sociologue de l'enfance et de l'alimentation. Elle travaille sur la socialisation et l'éducation des enfants et des jeunes au prisme de leurs pratiques alimentaires en milieu scolaire et familial. Elle est associée au laboratoire Printemps (UVSQ, CNRS UMR-8085) et au Centre Maurice Halbwachs (ENS, CNRS UMR-8097). Contact : geraldine.comoretto@gmail.com 
Nicole Darmon est directrice de recherche à l'INRAE (UMR MoISA). Experte en nutrition et santé publique, elle conduit des recherches observationnelles, des modélisations et des interventions sur la sécurité alimentaire durable. Contact : nicole.darmon@inrae.fr

Diane R. A. Djossinou est docteure en nutrition et sciences alimentaires et assistante de recherche à la faculté des Sciences agronomiques de l'université d'Abomey-Calavi (FSA/UAC) au Bénin. Elle travaille sur la consommation alimentaire et la composition nutritionnelle des aliments et recettes. Elle a travaillé lors de sa thèse sur l'alimentation des femmes avant et pendant la grossesse. Contact : dianedjossinou@gmail.com

Anne Dupuy est maîtresse de conférences en sociologie, responsable du master Sciences Sociales Appliquées à l'Alimentation (SSAA) à l'ISTHIA, de l'axe de recherche Santé et Alimentation au CERTOP/UMR-CNRS 5044 et directrice adjointe du Groupement d'intérêt scientifique Bébé, Petite Enfance en Contextes. Contact : anne.dupuy@univ-tlse2.fr

Muriel Figuié est sociologue et directrice de recherche au Cirad (MoISA). Ses recherches portent sur les risques sanitaires liés à l'animal dans l'élevage et l'alimentation et sur la santé globale, en lien avec les enjeux de développement dans les pays sous régime d'aide. Contact : muriel.figuie@cirad.fr

Estelle Fourat est sociologue. Ses recherches portent sur les dynamiques sociales et culturelles à l'œuvre dans la transformation des systèmes alimentaires vers plus de durabilité, incluant notamment des enjeux d'inclusion sociale et de consommations. Contact : efourat@gmail.com

Tristan Fournier est sociologue et chargé de recherche au CNRS (Iris - EHESS). Ses travaux portent sur les enjeux sociaux, politiques et moraux des changements alimentaires.

Contact : tristan.fournier@ehess.fr

Michelle Holdsworth est nutritionniste en santé publique et chercheuse à l'IRD (MoISA). Elle s'intéresse aux transformations des environnements alimentaires et son impact sur la consommation et les pratiques alimentaires, surtout en Afrique. Contact : michelle.holdsworth@ird.fr

Frédérique Jankowski est socio-anthropologue des connaissances, elle développe depuis plusieurs années des démarches performatives mobilisant la photographie, la vidéo et le théâtre participatif pour saisir les dimensions sensibles et éthiques de la relation à l'environnement.

Contact : frederique.jankowski@cirad.fr

Edwige Landais est nutritionniste de santé publique et s'intéresse aux enquêtes de consommation alimentaire menées en contexte Sud, avec comme objectif l'évaluation des régimes alimentaires et leurs déterminants. Contact : edwige.landais@ird.fr

Mila Lebrun est chercheuse en sciences sociales appliquées à l'alimentation et la santé. Sa thèse, réalisée au Cirad, portait sur le bien-être alimentaire de populations en insécurité alimentaire. Elle est actuellement responsable des évaluations d'impact du Fonds Danone pour l'écosystème. Contact : lebrun.mila@gmail.com

Olivier Lepiller est sociologue et chercheur au Cirad (UMR MoISA). Il s'intéresse aux transformations des normes et pratiques alimentaires en lien avec les enjeux sanitaires, marchands et d'industrialisation de l'alimentation. Contact : olivier.lepiller@cirad.fr

Alissia Lourme-Ruiz est chercheure en économie au Cirad (MoISA). Elle travaille principalement sur les déterminants de l'insécurité alimentaire et nutritionnelle des ménages agricoles dans les pays du Sud. Contact : alissia.lourme-ruiz@cirad.fr

Caroline Méjean est directrice de recherche à l'INRAE (MoISA) à Montpellier. Chercheure en épidémiologie et nutrition publique, ses recherches portent sur les interactions entre environnement alimentaire et durabilité des comportements alimentaires, sur les déterminants et effets des transitions nutritionnelles et alimentaires, et sur le nexus agriculture-alimentation.

Contact : caroline.mejean@inrae.fr

Margalida Mulet Pascual est docteure en anthropologie de l'EHESS et enseignante à la Universitat de les Illes Balears et au Barcelona Culinary Hub. Membre de l'Odela (université de Barcelone), elle s'intéresse aux aspects socioculturels de l'alimentation et à ses liens avec l'économie circulaire et l'agroécologie. Contact : margamp15@gmail.com 
Laurent Muller est directeur de recherche à l'NRAE (GAEL) à Grenoble. Il développe et utilise des méthodes d'économie expérimentale pour étudier les déterminants économiques des comportements de consommation finale alimentaire et leur inflexion par des politiques publiques incitatives. Contact : laurent.muller@inrae.fr

Marlène Perignon est ingénieure de recherche en nutrition publique à l'INRAE. Ses travaux portent sur l'étude des leviers d'amélioration de la durabilité de l'alimentation par des approches épidémiologiques, de modélisation (optimisation sous contraintes) et l'évaluation d'impact d'interventions visant à réduire l'insécurité alimentaire et promouvoir une alimentation plus durable. Contact : marlene.perignon@inrae.fr

Nguyen Thi Sau travaille au département d'Économie et de marketing du Fruit and Vegetable Research Institute (FAVRI), Vietnam. Elle est diplômée d'un master en économie, avec une spécialisation sur les marchés, la consommation et les chaînes de valeur.

Contact : nguyenthisau.hd@gmail.com

Nguyen Thi Tan Loc dirige le département d'Économie et de marketing du Fruit and Vegetable Research Institute (FAVRI), Vietnam. Diplômée d'un doctorat en économie agricole, elle possède une expérience de trente ans dans ce domaine. Contact : locnew259@gmail.com

Rebecca Pradeilles est chargée de recherche en nutrition publique à l'université de Loughborough (Royaume-Uni). Ses recherches portent sur la compréhension des facteurs des régimes alimentaires défavorables à la santé et de formes multiples de malnutrition qui co-existent dans les pays à revenu faible et intermédiaire. Contact : R.Pradeilles@lboro.ac.uk

Amandine Rochedy est sociologue, maîtresse de conférences à l'université Toulouse Jean-Jaurès (ISTHIA-CERTOP, UMR CNRS 5044). Ses travaux portent sur les pratiques alimentaires des enfants et adolescents en population générale et spécifique (autisme et syndrome de Prader-Willi) et leur gestion par l'entourage familial. Contact : amandine.rochedy@univ-tlse2.fr

Ophélie Roudelle est chargée de mission à la Chaire Unesco Alimentations du monde à Montpellier. Elle est en charge de la diffusion, de la communication et de la promotion du dialogue sciencesociété du projet de recherche Urbal. Contact : ophelie.roudelle@gmail.com

Denis Sautier est chercheur en économie agroalimentaire au Cirad (Montpellier, France). Il est spécialiste des systèmes agroalimentaires localisés. Il travaille également sur la labellisation territoriale des aliments et les indications géographiques. Contact : denis.sautier@cirad.fr

Mathilde Savy est docteure en épidémiologie et chercheure en nutrition publique à l'IRD. Elle travaille sur l'insécurité alimentaire et nutritionnelle en Afrique. Contact : mathilde.savy@ird.fr

Pauline Scherer est sociologue-intervenante et travaille en recherche-action sur la précarité alimentaire et les inégalités d'accès à l'alimentation durable. Elle est associée au Léris, Laboratoire d'étude et de recherche sur l'intervention sociale à Montpellier. Contact : pauline.scherer@gmail.com

Christophe Serra-Mallol est maître de conférences en sociologie à l'université Toulouse JeanJaurès (CERTOP, UMR CNRS 5044). Il s'intéresse aux aspects socioculturels de l'alimentation et ses liens avec l'identité, la santé et le bien-être dans les territoires ultramarins français du Pacifique. Contact : christophe.serra-mallol@univ-tlse2.fr

Sabrina Teyssier est chargée de recherche à l'INRAE, spécialisée en économie comportementale et expérimentale. Ses travaux s'intéressent aux mécanismes conduisant à des changements de comportements en faveur d'une alimentation plus durable. Contact : sabrina.teyssier@inrae.fr

Marion Tharrey est jeune docteure en nutrition et en santé publique à l'UMR MoISA. Elle s'intéresse au rôle de l'environnement alimentaire et physique sur la santé, notamment dans un contexte urbain. Contact : mariontharrey@hotmail.fr

Élodie Valette est géographe et chercheure au Cirad (Art-Dev). Elle mène des recherches sur les interactions villes, agricultures et alimentation dans une perspective territoriale.elodie.valette@cirad.fr

Amélie Wood est doctorante au Cirad (MoISA) et à l'université Gustave-Eiffel (LISIS). Sa thèse porte sur les liens entre la recherche en nutrition et les politiques publiques nutritionnelles, les relations entre acteurs et les déterminants de politique économique influençant les processus décisionnels dans un contexte d'Afrique subsaharienne. Contact : amelie.v.wood@protonmail.com 
Préparation éditoriale : Marie-Laure Portal

Mise en page : Hélène Bonnet Studio 9

Imprimeur : ISIPrint

Dépôt legal : novembre 2021 
Les chercheurs donnent rarement à voir le détail des choix méthodologiques qui fondent leurs recherches. Les considérations théoriques et pratiques qui les ont guidés dans ces choix restent souvent implicites. Dans cet ouvrage, les méthodes pour étudier l'alimentation et les « mangeurs » sont au centre. L'usage du terme « mangeurs » vise à distinguer ces derniers de la figure du consommateur. Il met l'accent sur la multidimensionnalité de l'acte de manger, cet acte qui engage le corps biologique tout autant que la socialité des individus et les insère dans l'espace et le temps ainsi que dans des échanges économiques. Cet ouvrage est le fruit d'un travail collectif réunissant une quarantaine de chercheuses et chercheurs confirmés. Soucieux de pédagogie, il a pour objectif de présenter de manière critique quinze méthodes aujourd'hui utilisées dans ou à la croisée de différentes disciplines : anthropologie, économie, géographie, nutrition et sociologie. L'ouvrage intéressera étudiants, enseignants, chercheurs experts et autres professionnels à la recherche de méthodes permettant de mieux comprendre ou d'affiner leurs propres outils d'étude de l'alimentation et des mangeurs.

Olivier Lepiller est sociologue et chercheur au Cirad (MoISA). Il s'intéresse aux transformations des normes et pratiques alimentaires en lien avec les enjeux sanitaires, marchands et d'industrialisation de l'alimentation.

Tristan Fournier est sociologue et chargé de recherche au CNRS (Iris - EHESS). Ses travaux portent sur les enjeux sociaux, politiques et moraux des changements alimentaires.

Nicolas Bricas est chercheur en socio-économie de l'alimentation au Cirad (MoISA), titulaire de la Chaire Unesco Alimentations du monde et co-directeur du Mastère Spécialisé « Innovations et Politiques pour une Alimentation Durable (MS IPAD) ».

Muriel Figuié est sociologue et directrice de recherche au Cirad (MolSA). Ses recherches portent sur les risques sanitaires liés à l'animal dans l'élevage et l'alimentation, et sur la santé globale, en lien avec les enjeux de développement dans les pays sous régime d'aide.

En couverture : dessin de Benjamin Delande, 2021.

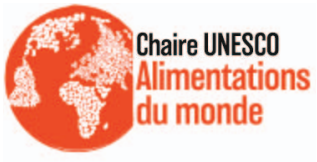

L'ÉCOLE DES HAUTES SCIENCES SOCIALES
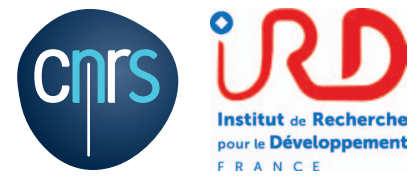

$29 €$

ISBN : 978-2-7592-3346-5

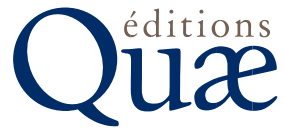

Éditions Cirad, Ifremer, INRAE www.quae.com
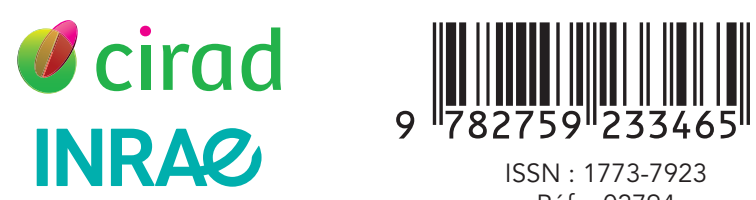

ISSN : 1773-7923

Réf. : 02794 